\title{
Drell-Yan angular lepton distributions at small $x$ from TMD factorization.
}

\section{Ian Balitsky}

Physics Department, Old Dominion University, Norfolk, VA 23529, U.S.A.

U.S.A. and Thomas Jefferson National Accelerator Facility, Newport News, VA 23606, U.S.A.

E-mail: balitsky@jlab.org

ABSTRACT: The Drell-Yan process is studied in the framework of TMD factorization in the Sudakov region $s \gg Q^{2} \gg q_{\perp}^{2}$ corresponding to recent LHC experiments with $Q^{2}$ of order of mass of Z-boson and transverse momentum of DY pair $\sim$ few tens $\mathrm{GeV}$. The DY hadronic tensors are expressed in terms of quark and quark-gluon TMDs with $\frac{1}{Q^{2}}$ and $\frac{1}{N_{c}^{2}}$ accuracy. It is demonstrated that in the leading order in $N_{c}$ the higher-twist quark-quark-gluon TMDs reduce to leading-twist TMDs due to QCD equation of motion. The resulting hadronic tensors depend on two leading-twist TMDs: $f_{1}$ responsible for total DY cross section, and Boer-Mulders function $h_{1}^{\perp}$. The corresponding qualitative and semi-quantitative predictions seem to agree with LHC data on five angular coefficients $A_{0}-A_{4}$ of DY pair production. The remaining three coefficients $A_{5}-A_{7}$ are determined by quark-quark-gluon TMDs multiplied by extra $\frac{1}{N_{c}}$ so they appear to be relatively small in accordance with LHC results.

KEYWORDS: Perturbative QCD, Nonperturbative Effects

ArXiv EPrint: 2105.13391 


\section{Contents}

1 Introduction 1

2 Drell-Yan cross section in the Sudakov region 3

3 TMD factorization from rapidity factorization 5

4 Hadronic tensor for photon-mediated DY process 4

$\begin{array}{lll}4.1 & W^{\mathrm{F}} \text { contribution } & 10\end{array}$

4.1.1 Terms with one quark-quark-gluon TMD (one-gluon terms) 11

4.1.2 Terms with two quark-quark-gluon TMDs (two-gluon terms) 12

$\begin{array}{lll}4.2 W^{\mathrm{G}} \text { term of eq. (4.8) } & 14\end{array}$

$\begin{array}{lll}4.3 W^{\mathrm{T}} \text { contribution of eq. (4.9) } & 15\end{array}$

$\begin{array}{lll}\text { 4.3.1 } & \text { One-gluon terms in } \check{V}_{\mu \nu}^{\mathrm{H}}(x) & 16\end{array}$

$\begin{array}{lll}\text { 4.3.2 Two-gluon terms in } \breve{V}_{\mu \nu}^{\mathrm{H}}(x) & 17\end{array}$

4.4 Exchange-type power corrections from $J_{A}^{\mu}(x) J_{B}^{\nu}(0)$ terms 21

4.5 Resulting hadronic tensor for photon-mediated DY process 22

$5 \quad Z$-mediated hadronic tensor $\quad \mathbf{2 3}$

5.1 Symmetric part of $Z$-mediated hadronic tensor 23

5.1.1 Exchange-type power corrections to $W_{\mu \nu}^{Z S} \quad 25$

5.1.2 Results for symmetric hadronic tensor for Z-mediated DY process 27

5.2 Antisymmetric part of $Z$-boson hadronic tensor 28

$\begin{array}{lll}5.2 .1 & \text { One-gluon terms in } \mathcal{W}_{\mu \nu}^{\mathcal{F}} & 30\end{array}$

$\begin{array}{lll}5.2 .2 & \text { Two-gluon terms in } \mathcal{W}_{\mu \nu}^{\mathcal{F}} & 32\end{array}$

5.2.3 Sum of $\mathcal{W}_{\mu \nu}^{\mathcal{F}}$ terms 33

$\begin{array}{lll}5.2 .4 \mathcal{W}_{\mu \nu}^{\mathrm{f5}} \text { contribution } & 34\end{array}$

$\begin{array}{lll}5.3 W_{\mu \nu}^{\text {as }} & 35\end{array}$

$\begin{array}{lll}\text { 5.3.1 One-gluon terms } & 35\end{array}$

$\begin{array}{lll}\text { 5.3.2 Two-gluon terms } & 37\end{array}$

5.3.3 Exchange-type power corrections to $W_{\mu \nu}^{\mathrm{ZA}} \quad 39$

5.4 Results for antisymmetric hadronic tensor for Z-mediated DY process 41

6 Hadronic tensors for interference terms $\quad 42$

6.1 Symmetric part of interference hadronic tensor $W^{\mathrm{I} 1} \quad 42$

6.1.1 Annihilation-type terms 42

6.1.2 Exchange-type power corrections 43

6.1.3 The result for the symmetric part of $W_{\mu \nu}^{\mathrm{I1}}(x) \quad 43$

6.2 Antiymmetric part of interference hadronic tensor $W^{\mathrm{I} 1}$

6.2.1 The result for antisymmetric part of interference hadronic tensor $W^{\mathrm{I} 1} 45$

6.3 Symmetric part of interference hadronic tensor $W^{\mathrm{I} 2} \quad 45$

6.3.1 Annihilation-type power corrections 45 
$\begin{array}{lll}\text { 6.3.2 } & \text { One-gluon terms } & 47\end{array}$

6.3.3 Two-gluon terms $\quad 51$

6.3.4 Exchange-type power corrections 54

6.3.5 Result for symmetric interference term $W^{\mathrm{I} 2 \mathrm{san}} \quad 54$

6.4 Antisymmetric interference term of tensor $W^{\mathrm{I} 2}$

6.4.1 Annihilation-type power corrections 55

6.4.2 Exchange-type power corrections and the result for $W^{\mathrm{I} 2} \quad 57$

7 Results $\quad \mathbf{5 8}$

7.1 Hadronic tensors in Collins-Soper frame 58

7.1.1 Hadronic tensor for photon-mediated DY process 58

$\begin{array}{lll}\text { 7.1.2 Hadronic tensor for } Z \text {-mediated DY process } & 59\end{array}$

$\begin{array}{lll}7.1 .3 & \text { Interference tensors } & 59\end{array}$

$\begin{array}{ll}\text { 7.1.4 Angular coefficients } & 60\end{array}$

$\begin{array}{lll}7.2 & \text { Comparison with LHC measurements } & 61\end{array}$

7.2.1 Logarithmical estimates of $\mathcal{W}_{i}$ at $Q_{\perp}^{2} \gg m^{2} \quad 62$

7.2.2 Estimates of $A_{0}$ and $A_{2} \quad 63$

$\begin{array}{lll}\text { 7.2.3 } & \text { Qualitative checks for other angular coefficients } & 64\end{array}$

8 Conclusions and outlook $\quad 64$

$\begin{array}{lc}\text { A Frequently used formulas } & \mathbf{6 6}\end{array}$

A.1 Formulas with Dirac matrices $\quad 66$

A.1.1 Fierz transformations $\quad 66$

$\begin{array}{lll}\text { A.1.2 Formulas with } \sigma \text {-matrices } & 67\end{array}$

A.1.3 Formulas with $\gamma$-matrices and one gluon field 68

$\begin{array}{lll}\text { A.1.4 Formulas with } \gamma \text {-matrices and two gluon fields } & 68\end{array}$

$\begin{array}{lll}\text { A.2 Parametrization of leading-twist matrix elements } & 71\end{array}$

A.3 Matrix elements of quark-quark-gluon operators 73

$\begin{array}{lll}\text { A.4 Matrix elements for exchange-type power corrections } & 76\end{array}$

$\begin{array}{lll}\text { A.5 Products of leptonic tensor and hadronic tensors' structures } & 79\end{array}$

\section{Introduction}

The Drell-Yan (DY) process [1] has been extensively studied in high energy physics field for precise tests of QCD, investigation of the structure of the proton, and searches for possible new physics. Since the advent of high-energy colliders, the attention shifted to processes with large invariant mass of DY pair produced both by photon and $Z$-boson. The important part of these studies is the transverse-momentum dependence of angular distribution of DY lepton pairs with 1 arge invariant mass produced in hadronic collisions. It was extensively studied in the framework of collinear factorization [2-7] leading to good agreement with 
experiment [8]. If one considers, however, the DY process at transverse momentum of lepton pair much smaller than their invariant mass, the collinear factorization should be replaced by TMD factorization [9-13]. In this paper the DY process will be studied in so-called Sudakov plus small- $x$ region $s \gg Q^{2} \gg q_{\perp}^{2}$ where $Q^{2}$ is the invariant mass of DY pair and $q_{\perp}$ is the transverse momentum of produced leptons. The typical case is the lepton pair production at LHC with DY invariant mass in the vicinity of $Z$-boson and transverse momentum of DY pair of order of ten or few tens of GeV.

This paper is the third in a series of papers devoted to description of DY process in terms of TMD rapidity factorization. In the first paper [14], A. Tarasov and the author calculated power corrections to the total cross section of $Z$-boson production using the method developed in earlier paper [15]. The second paper [16] was devoted to calculation of angular coefficients for DY process mediated by virtual photon. Unfortunately, it is hard to compare these results with experiment due to the fact that the LHC measurements of angular distributions are performed at the invariant mass $\sim 100 \mathrm{GeV}$ where the contribution of $Z$-boson is dominant. The present paper is devoted to generalization of the approach of papers [14] and [16] to angular distributions of DY pair production by unpolarized protons at LHC kinematics.

The differential cross section of DY process is determined by the sum of products of leptonic tensors and hadronic tensors. The leptonic tensors are given by simple first-order EW diagrams while hadronic tensors are determined by QCD correlation functions

$$
W_{\mu \nu}(q)=\frac{1}{(2 \pi)^{4}} \int d^{4} x e^{-i q x}\left\langle p_{A}, p_{B}\left|j_{\mu}(x) j_{\nu}(0)\right| p_{A}, p_{B}\right\rangle .
$$

where $p_{A}, p_{B}$ are hadron momenta, $q$ is the momentum of DY pair, and $j_{\mu}$ is either electromagnetic or $Z$-boson current.

As was mentioned above, a golden standard of QCD analysis of such hadronic tensors in the region where transverse momenta are much smaller than the invariant mass of the DY pair is the TMD factorization. The leading-twist hadronic tensors can be represented as

$$
\begin{aligned}
W_{i}= & \sum_{\text {flavors }} e_{f}^{2} \int d^{2} k_{\perp} \mathcal{D}_{f / A}^{(i)}\left(x_{A}, k_{\perp}\right) \mathcal{D}_{f / B}^{(i)}\left(x_{B}, q_{\perp}-k_{\perp}\right) C_{i}\left(q, k_{\perp}\right) \\
& + \text { power corrections }+\mathrm{Y}-\text { terms }
\end{aligned}
$$

where $\mathcal{D}_{f / A}\left(x_{A}, k_{\perp}\right)$ is the TMD density of a parton $f$ in hadron $A$ with fraction of momentum $x_{A}$ and transverse momentum $k_{\perp}, \mathcal{D}_{f / B}\left(x_{B}, q_{\perp}-k_{\perp}\right)$ is a similar quantity for hadron $B$, and coefficient functions $C_{i}(q, k)$ are determined by the cross section $\sigma\left(f f \rightarrow \mu^{+} \mu^{-}\right)$ of production of DY pair of invariant mass $q^{2}$ in the scattering of two partons. The DY angular distributions are conventionally parametrized by eight angular coefficients $A_{i}$ in Collins-Soper frame [2]

$$
\begin{aligned}
\frac{d \sigma}{d Q^{2} d y d \Omega_{l}}= & \frac{3}{16 \pi} \frac{d \sigma}{d Q^{2} d y}\left[\left(1+c_{\theta}^{2}\right)+\frac{A_{0}}{2}\left(1-3 c_{\theta}^{2}\right)+A_{1} s_{2 \theta} c_{\phi}+\frac{A_{2}}{2} s_{\theta}^{2} c_{2 \phi}\right. \\
& \left.+A_{3} s_{\theta} c_{\phi}+A_{4} c_{\theta}+A_{5} s_{\theta}^{2} s_{2 \phi}+A_{6} s_{2 \theta} s_{\phi}+A_{7} s_{\theta} s_{\phi}\right]
\end{aligned}
$$


where $y$ is the rapidity of DY pair and $c_{\phi} \equiv \cos \phi, s_{\phi} \equiv \sin \phi$ etc. The aim of this paper is to express $A_{i}\left(Q^{2}, q_{\perp}^{2}\right)$ at Sudakov kinematics $s \gg Q^{2} \gg q_{\perp}^{2}$ in terms of TMDs and compare (at least qualitatively) to ATLAS [17] and CMS [18] measurements.

Unfortunately, the TMD analysis of Drell-Yan angular distributions $A_{i}$ is hindered by the fact that not all hadronic tensors are determined by leading-twist quark-antiquark TMDs which have parton interpretation. Some tensor structures in the r.h.s. of eq. (1.1) are determined by power corrections to leading-twist TMDs expressed in terms of quarkantiquark-gluon distributions which are virtually unknown. Fortunately, as demonstrated recently in ref. [14], at the leading order in $N_{c}$ these power corrections are still determined by leading-twist TMDs. Moreover, at small $x_{A}$ and $x_{B}$ the majority of hadronic tensors depends only on two leading-twist TMDs: $f_{1}$ responsible for total DY cross section, and Boer-Mulders function $h_{1}^{\perp}$. The rest of hadronic tensors determined by power corrections due to quark-antiquark-gluon distributions are down by at least one $\frac{1}{N_{c}}$ factor which seems to qualitatively agree with LHC measurements of angular distributions.

Note that in addition to power corrections due to QCD dynamics, there are fiducial power corrections arising from fiducial cuts on experimental measurements. These cuts introduce linear power corrections in $q_{\perp} / Q$, see the discussion in ref. [19]. In this paper we do not consider fiducial power corrections.

The paper is organized as follows. In section 2I set up the notations, present the formula for differential cross section of DY lepton pair, and list the relevant hadronic tensors. In section 3I briefly outline the method of calculation of power corrections to hadronic tensors developed in refs. [14] and [15]. Section 4 contains the streamlined calculation of photon-mediated DY process which is used as a reference point to calculation of $Z$ mediated and interference terms in sections 5 and 6 . The results for hadronic tensors and angular coefficients are presented in section 7 whic also contains the comparison to LHC measurements. Conclusions are summarized in section 8 and the necessary technical details are listed in the appendix.

\section{Drell-Yan cross section in the Sudakov region}

At high energies, the production of a neutral $e^{+} e^{-}\left(\right.$or $\left.\mu^{+} \mu^{-}\right)$pair in hadron-hadron collisions is mediated by virtual photon or by $Z$-boson, see figure 1

$$
h_{A}\left(p_{A}\right)+h_{B}\left(p_{B}\right) \rightarrow \gamma, Z(q)+X \rightarrow l_{1}(l)+l_{2}\left(l^{\prime}\right)+X,
$$

where $h_{A, B}$ denote the colliding hadrons with momenta $p_{A}$ and $P_{B}$ and $l_{1,2}$ denote the outgoing lepton pair with total momentum $q=l+l^{\prime}$. To avoid cluttering of $\mu$ 's if our formulas, we will consider production of $e^{+} e^{-}$pairs, the results for $\mu^{+} \mu^{-}$pairs are the same.

The relevant terms of the Lagrangian for quark fields $\psi^{f}$ are

$$
\begin{aligned}
\mathcal{L}_{\gamma} & =e \int d^{4} x J_{\mu} A^{\mu}(x), & J_{\mu} & =\bar{e} \gamma_{\mu} e-\sum_{\text {flavors }} e_{f} \bar{\psi}^{f} \gamma_{\mu} \psi^{f} \\
\mathcal{L}_{Z} & =e \int d^{4} x \mathcal{J}_{\mu} Z^{\mu}(x), & \mathcal{J}_{\mu} & =c_{e} \bar{e}\left(a_{e}-\gamma_{5}\right) e-\sum_{\text {flavors }} c_{f} \bar{\psi}^{f} \gamma_{\mu}\left(a_{f}-\gamma_{5}\right) \psi^{f}
\end{aligned}
$$




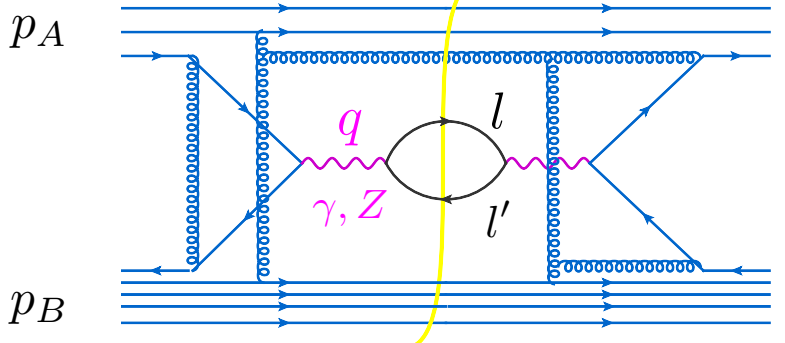

(a)

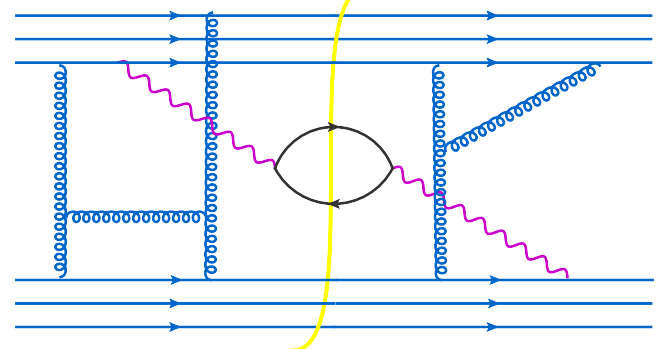

(b)

Figure 1. Typical annihilation-type (a) and exchange-type (b) diagrams for DY particle production.

where $e$ is the positron charge and

$$
\begin{aligned}
& c_{u, c}=\frac{1}{4 c_{W} s_{W}}, \quad a_{u, c}=1-\frac{8}{3} s_{W}^{2}, \quad c_{d, s}=-\frac{1}{4 c_{W} s_{W}}, \quad a_{d, s}=1-\frac{4}{3} s_{W}^{2}, \\
& c_{e}=\frac{1}{4 c_{W} s_{W}}, \quad a_{e}=1-4 s_{W}^{2}, \quad c_{W} \equiv \cos \theta_{W}, \quad s_{W} \equiv \sin \theta_{W} .
\end{aligned}
$$

The differential cross section of production of pair of leptons with momenta $l$ and $l^{\prime}$ by scattering of two unpolarized protons is given by

$$
\begin{aligned}
d \sigma= & \frac{d^{4} q}{2 s} \int \frac{d^{3} l d^{3} l^{\prime} \delta\left(q-l-l^{\prime}\right)}{(2 \pi)^{6} E_{l} E_{l}^{\prime}} e^{4} \int d x e^{-i q x}\left\langle p_{A}, p_{B}\right|\left[A^{\lambda} J_{\lambda}(x)+Z^{\lambda} \mathcal{J}_{\lambda}(x)\right] \\
& \times \int d x^{\prime} d y^{\prime}\left[A^{\mu} \bar{e} \gamma_{\mu} e\left(x^{\prime}\right)+c_{e} Z^{\mu} \bar{e} \gamma_{\mu}\left(a_{e}-\gamma_{5}\right) e\left(x^{\prime}\right)\right] \sum_{X}|X\rangle\langle X| \\
& \times \sum_{s, s^{\prime}}\left|l, s ; l^{\prime}, s^{\prime}\right\rangle\left\langle l, s ; l^{\prime}, s^{\prime}\right|\left[A^{\nu} \bar{e} \gamma_{\nu} e\left(x^{\prime}\right)+c_{e} Z^{\nu} \bar{e} \gamma_{\nu}\left(a_{e}-\gamma_{5}\right) e\left(x^{\prime}\right)\right] \\
& \times\left[A^{\rho} J_{\rho}(0)+Z^{\rho} \mathcal{J}_{\rho}(0)\right]\left|p_{A}, p_{B}\right\rangle
\end{aligned}
$$

where $\sum_{X}$ denotes summation over all intermediate hadron states. Performing contractions to get photon, Z-boson and lepton propagators, one obtains

$$
\begin{aligned}
d \sigma= & \frac{d^{4} q}{2 s} \int \frac{d^{3} l d^{3} l^{\prime} \delta\left(q-l-l^{\prime}\right)}{\pi^{2} E_{l} E_{l}^{\prime}} \frac{e^{4}}{N_{c}}\left[\frac{1}{q^{4}} L^{\mu \nu} W_{\mu \nu}(q)\right. \\
& +\frac{c_{e}^{2}}{\left|m_{Z}^{2}-q^{2}\right|^{2}+\Gamma_{Z}^{2} m_{Z}^{2}}\left[\left(a_{e}^{2}+1\right) L^{\mu \nu}-2 i a_{e} \epsilon^{\mu \nu \lambda \rho} l_{\lambda} l_{\rho}^{\prime}\right] W_{\mu \nu}^{Z}(q) \\
& +2 c_{e} \frac{\left(q^{2}-m_{Z}^{2}\right) / q^{2}}{\left|m_{Z}^{2}-q^{2}\right|^{2}+\Gamma_{Z}^{2} m_{Z}^{2}} W_{\mu \nu}^{\mathrm{I}}(q)\left[a_{e} L^{\mu \nu}-i \epsilon^{\mu \nu \lambda \rho} l_{\lambda} l^{\prime} \rho\right] \\
& +2 c_{e} \frac{i \Gamma_{Z} m_{Z} / q^{2}}{\left|m_{Z}^{2}-q^{2}\right|^{2}+\Gamma_{Z}^{2} m_{Z}^{2}} W_{\mu \nu}^{\mathrm{I} 2}(q)\left[a_{e} L^{\mu \nu}-i \epsilon^{\mu \nu \lambda \rho} l_{\lambda} l_{\rho}^{\prime}\right]
\end{aligned}
$$


where $L_{\mu \nu}=l_{\mu} l_{\nu}^{\prime}+l_{\mu}^{\prime} l_{\nu}-g_{\mu \nu} l \cdot l^{\prime}$ and hadronic tensors $W_{\mu \nu}^{i}$ are defined as

$$
\begin{aligned}
W_{\mu \nu}^{i}(q) & =\frac{1}{(2 \pi)^{4}} \int d^{4} x e^{-i q x} W_{\mu \nu}^{i}(x) \\
W_{\mu \nu}(x) & =N_{c}\left\langle A, B\left|J_{\mu}(x) J_{\nu}(0)\right| A, B\right\rangle \\
W_{\mu \nu}^{\mathrm{Z}}(x) & =N_{c}\left\langle A, B\left|\mathcal{J}_{\mu}(x) \mathcal{J}_{\nu}(0)\right| A, B\right\rangle \\
W_{\mu \nu}^{\mathrm{I} 1}(x) & =\frac{N_{c}}{2}\left\langle A, B\left|J_{\mu}(x) \mathcal{J}_{\nu}(0)+\mathcal{J}_{\mu}(x) J_{\nu}(0)\right| A, B\right\rangle \\
W_{\mu \nu}^{\mathrm{I} 2}(x) & =\frac{N_{c}}{2}\left\langle A, B\left|\mathcal{J}_{\mu}(x) J_{\nu}(0)-J_{\mu}(x) \mathcal{J}_{\nu}(0)\right| A, B\right\rangle
\end{aligned}
$$

Hereafter $\left|p_{A}, p_{B}\right\rangle \equiv|A, B\rangle$ for brevity. Note that hadronic tensors are defined with an extra $N_{c}$ so that the leading-twist contribution will be $\sim N_{c}^{0}$.

To convolute with leptonic tensors, we need to find symmetric and antisymmetric parts separately so we define

$$
\begin{array}{rlrl}
W_{\mu \nu}^{\mathrm{ZS}} & =\frac{1}{2}\left(W_{\mu \nu}^{\mathrm{Z}}+\mu \leftrightarrow \nu\right), & W_{\mu \nu}^{\mathrm{ZA}} & =\frac{1}{2}\left(W_{\mu \nu}^{\mathrm{Z}}-\mu \leftrightarrow \nu\right), \\
W_{\mu \nu}^{\mathrm{I} 1 \mathrm{~S}} & =\frac{1}{2}\left(W_{\mu \nu}^{\mathrm{I} 1}+\mu \leftrightarrow \nu\right), & W_{\mu \nu}^{\mathrm{I} 1 \mathrm{~A}}=\frac{1}{2}\left(W_{\mu \nu}^{\mathrm{I} 1}-\mu \leftrightarrow \nu\right), \\
W_{\mu \nu}^{\mathrm{I} 2 \mathrm{~S}}=\frac{1}{2}\left(W_{\mu \nu}^{\mathrm{I} 2}+\mu \leftrightarrow \nu\right), & W_{\mu \nu}^{\mathrm{I} A \mathrm{~A}}=\frac{1}{2}\left(W_{\mu \nu}^{\mathrm{I} 2}-\mu \leftrightarrow \nu\right) .
\end{array}
$$

We will calculate these hadronic tensors with $O\left(\frac{1}{Q^{2}}\right)$ accuracy and express them in terms of TMDs like eq. (1.2). It turns out that each $W^{i}$ is a sum of three parts

$$
W_{\mu \nu}^{i}(q)=\left(W_{1}\right)_{\mu \nu}^{i}(q)+\left(W_{2}\right)_{\mu \nu}^{i}(q)+\left(W^{\mathrm{ex}}\right)_{\mu \nu}^{i}(q)
$$

The first part is determined by leading-twist TMDs $f_{1}$ and $h_{1}^{\perp}$ as mentioned in the Introduction and satisfies the condition ${ }^{1}$

$$
q^{\mu}\left(W_{1}\right)_{\mu \nu}^{i}(q)=0
$$

The two remaining terms $\left(W_{2}\right)_{\mu \nu}^{i}$ and $\left(W^{\mathrm{ex}}\right)_{\mu \nu}^{i}$ are power corrections $\sim O\left(\frac{q_{\perp}^{2} g_{\mu \nu}^{\perp}}{Q^{2}}, \frac{q_{\mu}^{\perp} q_{\nu}^{\perp}}{Q^{2}}\right)$ which come from the diagrams of the figure 1a,b type, respectively. They are expressed in terms of quark-antiquark-gluon matrix elements which cannot be reduced to leadingtwist TMDs. The term $\left(W_{2}\right)_{\mu \nu}^{i}$ is $\sim \frac{q_{\perp}^{2}}{Q^{2}}$ and $\sim N_{c}^{0}$ as while $\left(W^{\mathrm{ex}}\right)_{\mu \nu}^{i}$ is $\sim \frac{1}{N_{c}}$. On the other hand, since $\left(W^{\mathrm{ex}}\right)_{\mu \nu}^{i}$ comes from exchange-type diagrams it may be numerically larger than $\left(W_{2}\right)_{\mu \nu}^{i}(q)$ coming from annihilation-type diagrams.

\section{TMD factorization from rapidity factorization}

We use Sudakov variables $p=\alpha p_{1}+\beta p_{2}+p_{\perp}$, where $p_{1}$ and $p_{2}$ are light-like vectors close to $p_{A}$ and $p_{B}$ so that $p_{A}=p_{1}+\frac{m^{2}}{s} p_{2}$ and $p_{A}=p_{1}+\frac{m^{2}}{s} p_{2}$ with $m$ being the proton mass.

\footnotetext{
${ }^{1}$ Strictly speaking, the Z-boson current is not conserved so one should not expect $q^{\mu}\left(W_{1}\right)_{\mu \nu}^{\mathrm{Z}, \mathrm{I}}(q)=0$. However, if we consider quarks to be massless, the non-conservation is due to axial anomaly so the corresponding terms in $q^{\mu}\left(W_{1}\right)_{\mu \nu}^{i}(q)$ will be proportional to $\left\langle p_{A}, p_{B}\left|\alpha_{s} F_{\mu \nu} \tilde{F}^{\mu \nu}(x) j_{\nu}(0)\right| p_{A}, p_{B}\right\rangle$. Such matrix elements will be non-zero only at the two-loop level $O\left(\alpha_{s}^{2}\right)$ which is beyond the accuracy of this paper.
} 


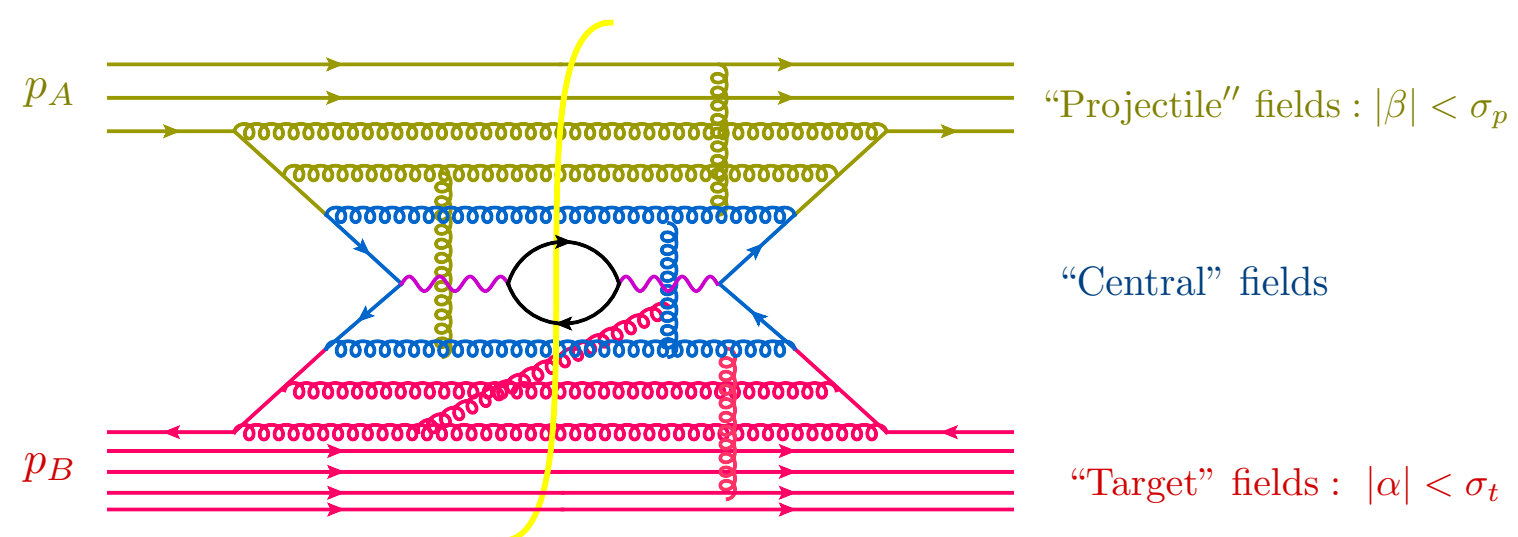

Figure 2. Rapidity factorization for DY particle production.

Also, we use the notations $x_{\bullet} \equiv x_{\mu} p_{1}^{\mu}$ and $x_{\star} \equiv x_{\mu} p_{2}^{\mu}$ for the dimensionless light-cone coordinates $\left(x_{\star}=\sqrt{\frac{s}{2}} x_{+}\right.$and $\left.x_{\bullet}=\sqrt{\frac{s}{2}} x_{-}\right)$. Our metric is $g^{\mu \nu}=(1,-1,-1,-1)$ which we will frequently rewrite as a sum of longitudinal part and transverse part:

$$
g^{\mu \nu}=g_{\|}^{\mu \nu}+g_{\perp}^{\mu \nu}=\frac{2}{s}\left(p_{1}^{\mu} p_{2}^{\nu}+p_{2}^{\mu} p_{1}^{\nu}\right)+g_{\perp}^{\mu \nu}
$$

Consequently, $p \cdot q=\left(\alpha_{p} \beta_{q}+\alpha_{q} \beta_{p}\right) \frac{s}{2}-(p, q)_{\perp}$ where $(p, q)_{\perp} \equiv-p_{i} q^{i}$. Throughout the paper, the sum over the Latin indices $i, j, \ldots$ runs over two transverse components while the sum over Greek indices $\mu, \nu, \ldots$ runs over four components as usual.

Following ref. [15] we separate quark and gluon fields into three sectors (see figure 2): "projectile" fields $A_{\mu}, \psi_{A}$ with $|\beta|<\sigma_{p}$, "target" fields $B_{\mu}, \psi_{B}$ with $|\alpha|<\sigma_{t}$ and "central rapidity" fields $C_{\mu}, \psi_{C}$ with $|\alpha|>\sigma_{t}$ and $|\beta|>\sigma_{p}$, see figure $2 .^{2}$ Our goal is to integrate over central fields and get the amplitude in the factorized form, i.e. as a product of functional integrals over $A$ fields representing projectile matrix elements (TMDs of the projectile) and functional integrals over $B$ fields representing target matrix elements (TMDs of the target). In the spirit of background-field method, we "freeze" projectile and target fields and get a sum of diagrams in these external fields. Since $|\beta|<\sigma_{p}$ in the projectile fields and $|\alpha|<\sigma_{t}$ in the target fields, at the tree level one can set with power accuracy $\beta=0$ for the projectile fields and $\alpha=0$ for the target fields - the corrections will be $O\left(\frac{m^{2}}{\sigma_{p} s}\right)$ and $O\left(\frac{m^{2}}{\sigma_{t}}\right)$. In the coordinate space, projectile fields depend on $x_{\bullet}$ and $x_{\perp}$ and target ones on $x_{\star}$ and $x_{\perp}$. Beyond the tree level, the integration over $C$ fields produces logarithms of the cutoffs $\sigma_{p}$ and $\sigma_{t}$ which match the corresponding logs in TMDs of the projectile and the target, see the discussion in ref. [16]

As discussed in ref. [16], central fields at the tree level are given by a set of Feynman diagrams with retarded propagators in background field $A+B$ and $\psi_{A}+\psi_{B}$, see figure $3 .^{3}$ The set of such "retarded" diagrams represent the solution of QCD equations of motion

\footnotetext{
${ }^{2}$ Although the kinematics is best suited for LHC collider, I call $A$ hadron "projectile" and $B$ hadron "target" for convenience.

${ }^{3}$ We take into account only $u, d, s, c$ quarks and consider them massless. In principle, one can include "massless" $b$-quark for $q_{\perp}^{2} \gg m_{b}^{2}$.
} 


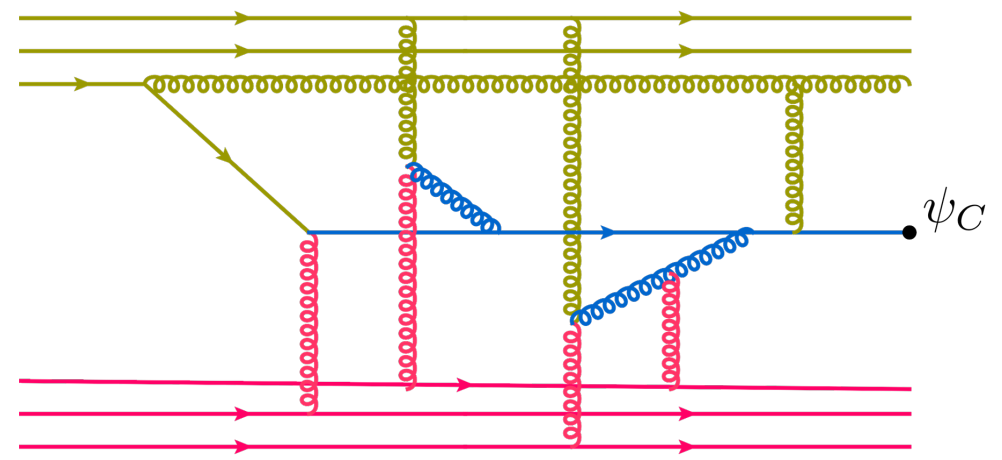

Figure 3. Typical diagram for the classical field with projectile/target sources. The Green functions of central fields are given by retarded propagators.

with sources being projectile and target fields. After summation of these diagrams the hadronic tensor (1.1) can be represented as

$$
W_{\mu \nu}=\frac{1}{(2 \pi)^{4}} \int d^{4} x e^{-i q x} \sum_{m, n} \int d z_{m} c_{m, n}(q, x)\left\langle p_{A}\left|\hat{\Phi}_{A}\left(z_{m}\right)\right| p_{A}\right\rangle \int d z_{n}^{\prime}\left\langle p_{B}\left|\hat{\Phi}_{B}\left(z_{n}^{\prime}\right)\right| p_{B}\right\rangle .
$$

where $c_{m, n}$ are coefficients and $\Phi$ can be any of the background fields promoted to operators after integration over projectile and target fields.

In general, the summation of diagrams of figure 3 type is a formidable task which still awaits its solution. Fortunately, as demonstrated in ref. [16], at our kinematics we have a small parameter $\frac{q_{\perp}^{2}}{Q^{2}} \ll 1$ and it is possible to expand classical solution for central fields in powers of this parameter.

Now we expand the classical quark and gluon fields in powers of $\frac{p_{\perp}^{2}}{p_{\|}^{2}} \sim \frac{m_{\perp}^{2}}{s}$. It is convenient to choose a gauge where $A_{\star}=0$ for projectile fields and $B_{\bullet}=0$ for target fields. (The existence of such gauge was proved in appendix B of ref. [15] by explicit construction.) As demonstrated in ref. [14], expanding it in powers of $p_{\perp}^{2} / p_{\|}^{2}$ we obtain

$$
\Psi(x)=\Psi_{1}(x)+\Psi_{2}(x)+\ldots,
$$

where

$$
\begin{array}{ll}
\Psi_{1}=\psi_{A}+\Xi_{1}, & \Xi_{1}=-\frac{\not p_{2}}{s} \gamma^{i} B_{i} \frac{1}{\alpha+i \epsilon} \psi_{A}=\frac{i}{s} \sigma_{\star i} B^{i} \frac{1}{\alpha+i \epsilon} \psi_{A}, \\
\bar{\Psi}_{1}=\bar{\psi}_{A}+\bar{\Xi}_{1}, & \bar{\Xi}_{1}=-\left(\bar{\psi}_{A} \frac{1}{\alpha-i \epsilon}\right) \gamma^{i} B_{i} \frac{\not p_{2}}{s}=-\frac{i}{s}\left(\bar{\psi}_{A} \frac{1}{\alpha-i \epsilon}\right) B^{i} \sigma_{\bullet} \\
\Psi_{2}=\psi_{B}+\Xi_{2}, & \Xi_{2}=-\frac{\not p_{1}}{s} \gamma^{i} A_{i} \frac{1}{\beta+i \epsilon} \psi_{B}=\frac{i}{s} \sigma_{\bullet i} A^{i} \frac{1}{\beta+i \epsilon} \psi_{B}, \\
\bar{\Psi}_{2}=\bar{\psi}_{B}+\bar{\Xi}_{2}, & \bar{\Xi}_{2}=-\left(\bar{\psi}_{B} \frac{1}{\beta-i \epsilon}\right) \gamma^{i} A_{i} \frac{\not p_{1}}{s}=-\frac{i}{s}\left(\bar{\psi}_{B} \frac{1}{\beta-i \epsilon}\right) A_{i} \sigma_{\bullet}
\end{array}
$$


and dots stand for terms subleading in $\frac{q_{\perp}^{2}}{Q^{2}}$ and/or $\alpha_{q}, \beta_{q}$ parameters. In this formula

$$
\begin{aligned}
\frac{1}{\alpha+i \epsilon} \psi_{A}\left(x_{\bullet}, x_{\perp}\right) & \equiv-i \int_{-\infty}^{x_{\bullet}} d x_{\bullet}^{\prime} \psi_{A}\left(x_{\bullet}^{\prime}, x_{\perp}\right), \\
\left(\bar{\psi}_{A} \frac{1}{\alpha-i \epsilon}\right)\left(x_{\bullet}, x_{\perp}\right) & \equiv i \int_{-\infty}^{x_{\bullet}} d x_{\bullet}^{\prime} \bar{\psi}_{A}\left(x_{\bullet}^{\prime}, x_{\perp}\right)
\end{aligned}
$$

and similarly for $\frac{1}{\beta \pm i \epsilon}$. For brevity, in what follows we denote $\left(\bar{\psi}_{A} \frac{1}{\alpha}\right)(x) \equiv\left(\bar{\psi}_{A} \frac{1}{\alpha-i \epsilon}\right)(x)$ and $\left(\bar{\psi}_{B} \frac{1}{\beta}\right)(x) \equiv\left(\bar{\psi}_{B} \frac{1}{\beta-i \epsilon}\right)(x)$. The corresponding expansion of classical gluon fields is presented in ref. [15], but we do not need it here. ${ }^{4}$

Let us estimate the relative size of corrections $\Xi$ in eq. (3.4) at small $x$. As we will see, $\frac{1}{\alpha}$ and $\frac{1}{\beta}$ transform to $\frac{1}{\alpha_{q}}$ and $\frac{1}{\beta_{q}}$ in our TMDs so

$$
\Xi_{1} \sim \psi_{A} \frac{m_{\perp}}{\alpha_{q} \sqrt{s}} \sim \psi_{A} \frac{q_{\perp}}{Q}, \quad \Xi_{2} \sim \psi_{B} \frac{m_{\perp}}{\beta_{q} \sqrt{s}} \sim \psi_{B} \frac{q_{\perp}}{Q}
$$

if $\alpha_{q} \sim \beta_{q} \sim \frac{Q}{\sqrt{s}}$ (recall that we assume that the DY pair is emitted in the central region of rapidity). For example, the correction $\sim\left[\bar{\psi}_{A} \gamma_{\mu} \Xi_{2}\right]\left[\bar{\psi}_{B} \gamma_{\nu} \Xi_{1}\right]$ will be of order of $\frac{q_{\perp}^{2}}{Q^{2}}$ in comparison to leading-twist contribution $\left[\bar{\psi}_{A} \gamma_{\mu} \psi_{B}\right]\left[\bar{\psi}_{B} \gamma_{\nu} \psi_{A}\right] .{ }^{5}$ As demonstrated in ref. [16], the relevant terms contributions to hadronic tensors (2.6) with this accuracy are

$$
\begin{array}{lll}
g_{\perp}^{\mu \nu}\left\{1, \frac{q_{\perp}^{2}}{\alpha_{q} \beta_{q} s}\right\}, & \frac{q_{\perp}^{\mu} q_{\perp}^{\nu}}{q_{\perp}^{2}}\left\{1, \frac{q_{\perp}^{2}}{\alpha_{q} \beta_{q} s}\right\}, & g_{\|}^{\mu \nu}\left\{1, \frac{q_{\perp}^{2}}{\alpha_{q} \beta_{q} s}\right\}, \\
\frac{1}{\beta_{q} s}\left(p_{1}^{\mu} q_{\perp}^{\nu} \pm p_{1}^{\nu} q_{\perp}^{\mu}\right), & \frac{1}{\alpha_{q} s}\left(p_{2}^{\mu} q_{\perp}^{\nu} \pm p_{2}^{\nu} q_{\perp}^{\mu}\right), & q_{\perp}^{2} \frac{p_{1}^{\mu} p_{1}^{\nu}}{\beta_{q}^{2} s^{2}}, \quad q_{\perp}^{2} \frac{p_{2}^{\mu} p_{2}^{\nu}}{\alpha_{q}^{2} s^{2}}
\end{array}
$$

Let us also specify the terms which we do not calculate. Roughly speaking, they correspond to terms in eq. (3.7) multiplied by $\frac{q_{\perp}^{2}}{Q^{2}}$ or by either $\alpha_{q}$ or $\beta_{q}$.

In addition, in this paper we will consider only leading- $N_{c}$ power corrections. As we will see below, leading-twist hadronic tensor is $\sim N_{c}^{0}$ and power corrections can be $\sim N_{c}^{0}$, $\sim \frac{1}{N_{c}}$, or $\frac{1}{N_{c}^{2}}$. The corrections $\sim \frac{1}{N_{c}^{2}}$ were found in ref. [14] for the case of total cross section, i.e. for eq. (2.5) integrated over $l$. In this paper such corrections $\sim \frac{1}{N_{c}^{2}}$ will be neglected.

Thus, the calculation of power corrections with our accuracy boils down to calculation of tensors (2.6) with $\psi \rightarrow \Psi_{1}+\Psi_{2}$. In the next sections we will consider five lines in eq. (2.5) for the differential cross section.

\section{Hadronic tensor for photon-mediated DY process}

In this section I briefly summarize the calculation of $W_{\mu \nu}^{f}$ performed in ref. [16] paying attention only to terms giving non-negligible contributions listed in eq. (3.7) at the leading- $N_{c}$

\footnotetext{
${ }^{4}$ Since we are dealing with tree approximation and quark equations of motion, it is convenient to include coupling constant $g$ in the definition of gluon fields.

${ }^{5}$ The reader may wonder why there are no corrections $\sim \frac{q_{\perp}^{2}}{Q^{2}}$ coming from next terms in the expansion (3.3) like $\left[\bar{\psi}_{A}(x) \gamma_{\mu} \psi_{B}(x)\right]\left[\bar{\psi}_{B}(0) \gamma_{\nu} \frac{\gamma^{i}}{s} \not p_{2} \frac{1}{\beta} \frac{1}{\alpha} \gamma^{j} \partial_{i} B_{j} \Psi_{1}(0)\right]$. The reason is that $\frac{1}{\beta}$ between $\bar{\psi}_{B}(0)$ and $B_{j}(0)$ does not transform to $\frac{1}{\beta_{q}}$ and remains $\sim O(1)$, see the discussion in the appendix 8.3 .4 of ref. [14].
} 
level. The reason is that hadronic tensors listed in eq. (2.6) differ from $W_{\mu \nu}$ by replace$\operatorname{ment}(\mathrm{s}) \gamma_{\mu(\nu)} \rightarrow \gamma_{\mu(\nu)} \gamma_{5}$ and/or $\mu, \nu$ antisymmetrization instead of symmetrization. Both operations do not change power counting in $\frac{q_{\perp}^{2}}{Q^{2}}$ and $\alpha_{q}, \beta_{q}$ parameters so the calculation of the rest of the terms in (2.6) will be based on the calculation of (non-negligible) contributions to in $W_{\mu \nu}$ outlined in this section.

In this section we take into account hadronic tensor due to electromagnetic currents of $u, d, s, c$ quarks and consider these quarks to be massless. It is convenient to define coordinate-space hadronic tensor multiplied by $\frac{2}{s}$ (and denoted by extra "check" mark) as follows

$$
\begin{aligned}
& \check{W}_{\mu \nu}(x) \equiv \frac{2}{s}\left\langle p_{A}, p_{B}\left|J_{\mu}(x) J_{\nu}(0)\right| p_{A}, p_{B}\right\rangle \\
& W_{\mu \nu}(q)=\frac{s / 2}{(2 \pi)^{4}} \int d^{4} x e^{-i q x} \check{W}_{\mu \nu}(x) .
\end{aligned}
$$

For future use, let us also define the hadronic tensor in mixed representation: in momentum longitudinal space but in transverse coordinate space

$$
\begin{aligned}
W_{\mu \nu}(q) & =\int d^{2} x_{\perp} e^{i(q, x)_{\perp}} W_{\mu \nu}\left(\alpha_{q}, \beta_{q}, x_{\perp}\right), \\
W_{\mu \nu}\left(\alpha_{q}, \beta_{q}, x_{\perp}\right) & \equiv \frac{1}{(2 \pi)^{4}} \int d x_{\bullet} d x_{\star} e^{-i \alpha_{q} x_{\bullet}-i \beta_{q} x_{\star}} \check{W}_{\mu \nu}\left(x_{\bullet}, x_{\star}, x_{\perp}\right) .
\end{aligned}
$$

With the definition (4.1), power counting of contributions to $\check{W}_{\mu \nu}\left(x_{\bullet}, x_{\star}, x_{\perp}\right)$ will mirror that of $W_{\mu \nu}(q)$ terms without extra $\frac{1}{s}$ factor.

After integration over central fields in the tree approximation we obtain

$$
\check{W}_{\mu \nu}(x) \equiv N_{c} \frac{2}{s}\left\langle A, B\left|J_{\mu}\left(x_{\bullet}, x_{\star}, x_{\perp}\right) J_{\nu}(0)\right| A, B\right\rangle
$$

where

$$
\begin{aligned}
-J^{\mu} & =J_{1}^{\mu}+J_{2}^{\mu}+J_{12}^{\mu}+J_{21}^{\mu}, \\
J_{1}^{\mu} & =\sum_{f} e_{f} \bar{\Psi}_{1}^{f} \gamma^{\mu} \Psi_{1}^{f}, \quad J_{12}^{\mu}=\sum_{f} e_{f} \bar{\Psi}_{1}^{f} \gamma^{\mu} \Psi_{2}^{f}
\end{aligned}
$$

and similarly for $J_{2}^{\mu}$ and $J_{21}^{\mu}$. Here $\left\langle A, B\left|\mathcal{O}\left(\psi_{A}, A_{\mu}, \psi_{B}, B_{\mu}\right)\right| A, B\right\rangle$ denotes double functional integral over $A$ and $B$ fields which gives matrix elements between projectile and target states of eq. (3.2) type.

The leading-twist contribution to $W_{\mu \nu}(q)$ comes only from annihilation-type product $J_{12}^{\mu}(x) J_{21}^{\nu}(0)+1 \leftrightarrow 2$ while power corrections may come also from $J_{1}^{\mu}(x) J_{2}^{\nu}(0)+1 \leftrightarrow 2$. As demonstrated in ref. [16], power corrections from $J_{1}^{\mu}(x) J_{2}^{\nu}(0)$ terms are down by one power of $N_{c}$ in comparison to leading- $N_{c}$ terms. On the other hand, they come from exchange-type diagrams like figure $1 \mathrm{~b}$ so they are determined by product of two quark distributions (one with additional gluon) rather than from annihilation-type diagrams in figure 1a proportional to product of quark and antiquark distributions. 
We will first calculate annihilation-type contributions coming from $J_{12}^{\mu}(x) J_{21}^{\nu}(0)+1 \leftrightarrow 2$ terms. Since leptonic tensor $L_{\mu \nu}$ is symmetric, we consider

$$
\begin{aligned}
& \check{W}_{\mu \nu}^{\mathrm{a}}(x)=\frac{N_{c}}{s}\left\langle A, B\left|J_{12}^{\mu}(x) J_{21}^{\nu}(0)+J_{21}^{\mu}(x) J_{12}^{\nu}(0)+\mu \leftrightarrow \nu\right| A, B\right\rangle=\sum_{f} e_{f}^{2} \check{W}_{\mu \nu}^{f}(x), \\
& \check{W}_{\mu \nu}^{f}(x)=\frac{N_{c}}{s}\left\langle A, B\left|\left[\bar{\Psi}_{1}^{f}(x) \gamma_{\mu} \Psi_{2}^{f}(x)\right]\left[\bar{\Psi}_{2}^{f}(0) \gamma_{\mu} \Psi_{1}^{f}(0)\right]+\mu \leftrightarrow \nu\right| A, B\right\rangle+x \leftrightarrow 0
\end{aligned}
$$

After Fierz transformations (A.1) and (A.3) they can be sorted out as

$$
\begin{aligned}
\check{W}_{\mu \nu}^{f}(x)= & \check{W}_{\mu \nu}^{\mathrm{F} f}(q)+\check{W}_{\mu \nu}^{\mathrm{H} f}(q) \\
\check{W}_{\mu \nu}^{\mathrm{F}}(x)= & \frac{N_{c}}{2 s}\left(g_{\mu \nu} g^{\alpha \beta}-\delta_{\mu}^{\alpha} \delta_{\nu}^{\beta}-\delta_{\nu}^{\alpha} \delta_{\mu}^{\beta}\right)\langle A, B|\left[\bar{\Psi}_{1}^{m}(x) \gamma_{\alpha} \Psi_{1}^{n}(0)\right]\left[\bar{\Psi}_{2}^{n}(0) \gamma_{\beta} \Psi_{2}^{m}(x)\right] \\
& +\gamma_{\alpha} \otimes \gamma_{\beta} \leftrightarrow \gamma_{\alpha} \gamma_{5} \otimes \gamma_{\beta} \gamma_{5}|A, B\rangle+x \leftrightarrow 0 \\
\check{W}_{\mu \nu}^{\mathrm{H}}(x)= & \check{W}_{\mu \nu}^{\mathrm{G}}(x)+\check{W}_{\mu \nu}^{\mathrm{T}}(x) \\
\check{W}_{\mu \nu}^{\mathrm{G}}(x)= & -g_{\mu \nu} \frac{N_{c}}{2 s}\langle A, B|\left[\bar{\Psi}_{1}^{m}(x) \Psi_{1}^{n}(0)\right]\left[\bar{\Psi}_{2}^{n}(0) \Psi_{2}^{m}(x)\right] \\
& -\left[\bar{\Psi}_{1}^{m}(x) \gamma_{5} \Psi_{1}^{n}(0)\right]\left[\bar{\Psi}_{2}^{n}(0) \gamma_{5} \Psi_{2}^{m}(x)\right]|A, B\rangle+x \leftrightarrow 0 \\
\check{W}_{\mu \nu}^{\mathrm{T}}(x)= & \frac{N_{c}}{2 s}\left(\delta_{\mu}^{\alpha} \delta_{\nu}^{\beta}+\delta_{\nu}^{\alpha} \delta_{\mu}^{\beta}-\frac{1}{2} g_{\mu \nu} g^{\alpha \beta}\right) \\
& \left.\times\langle A, B|\left[\bar{\Psi}_{1}^{m}(x) \sigma_{\alpha \xi} \Psi_{1}^{n}(0)\right] \bar{\Psi}_{2}^{n}(0) \sigma_{\beta}^{\xi} \Psi_{2}^{m}(x)\right]|A, B\rangle+x \leftrightarrow 0
\end{aligned}
$$

for flavor $f$ which we are considering. As discussed in section $3, x_{\star}$ in projectile matrix elements is set to be zero and similarly for $x_{\bullet}=0$ in target matrix elements. To save space, we will often assume this instead of explicitly displaying.

In the remainder of this section we will outline calculation of leading power corrections to the above equations starting with $W^{\mathrm{F}}$ terms.

\section{$4.1 W^{\mathrm{F}}$ contribution}

As we discussed in section 3, to calculate (4.6) one needs to plug in $\Psi_{i}$ in the form (3.4). First, the leading-twist contribution is

$$
\begin{aligned}
\check{W}_{\mu \nu}^{\mathrm{F}, \mathrm{lt}}(x)= & \frac{N_{c}}{2 s}\left(g_{\mu \nu} g^{\alpha \beta}-\delta_{\mu}^{\alpha} \delta_{\nu}^{\beta}-\delta_{\nu}^{\alpha} \delta_{\mu}^{\beta}\right)\left\langle p_{A}, p_{B}\right|\left\{\left[\bar{\psi}_{A}^{m}(x) \gamma_{\alpha} \psi_{A}^{n}(0)\right]\left[\bar{\psi}_{B}^{n}(0) \gamma_{\beta} \psi_{B}^{m}(x)\right]\right. \\
& \left.+\gamma_{\alpha} \otimes \gamma_{\beta} \leftrightarrow \gamma_{\alpha} \gamma_{5} \otimes \gamma_{\beta} \gamma_{5}\right\}\left|p_{A}, p_{B}\right\rangle+x \leftrightarrow 0 \\
= & \frac{1}{2 s}\left(g_{\mu \nu} g^{\alpha \beta}-\delta_{\mu}^{\alpha} \delta_{\nu}^{\beta}-\delta_{\nu}^{\alpha} \delta_{\mu}^{\beta}\right)\left\langle\bar{\psi}(x) \gamma_{\alpha} \psi(0)\right\rangle_{A}\left\langle\bar{\psi}(0) \gamma_{\beta} \psi(x)\right\rangle_{B} \\
& +\left(\psi(0) \otimes \psi(x) \leftrightarrow \gamma_{5} \psi(0) \otimes \gamma_{5} \psi(x)\right\}+x \leftrightarrow 0
\end{aligned}
$$

Hereafter we use notations $\langle\mathcal{O}\rangle_{A} \equiv\left\langle p_{A}|\mathcal{O}| p_{A}\right\rangle$ and $\langle\mathcal{O}\rangle_{B} \equiv\left\langle p_{B}|\mathcal{O}| p_{B}\right\rangle$ for brevity. Using parametrizations (A.39) and (A.40) we can write down the corresponding contribution to $W_{\mu \nu}^{F}$ in the form

$$
W_{\mu \nu}^{\mathrm{F}, \mathrm{lt}}(q)=\frac{1}{16 \pi^{4}} \int d x_{\bullet} d x_{\star} d^{2} x_{\perp} e^{-i \alpha_{q} x_{\bullet}-i \beta_{q} x_{\star}+i(q, x)_{\perp}} \check{W}_{\mu \nu}^{\mathrm{lt}}(x)=-g_{\mu \nu}^{\perp} \int d^{2} k_{\perp} F\left(q, k_{\perp}\right)
$$


where

$$
F^{f}\left(q, k_{\perp}\right)=f_{1}^{f}\left(\alpha_{q}, k_{\perp}\right) \bar{f}_{1}^{f}\left(\beta_{q},(q-k)_{\perp}\right)+f_{1}^{f} \leftrightarrow \bar{f}_{1}^{f}
$$

Here the term with $f \leftrightarrow \bar{f}$ comes from $x \leftrightarrow 0$ contribution.

\subsubsection{Terms with one quark-quark-gluon TMD (one-gluon terms)}

Next, here will be terms with one or two gluon fields in eq. (4.6) coming from replacement(s) (3.4). Terms with one gluon are

$$
\begin{aligned}
\check{W}_{\mu \nu}^{1 \mathrm{~F}}(x)= & \frac{N_{c}}{2 s}\left(g_{\mu \nu} g^{\alpha \beta}-\delta_{\mu}^{\alpha} \delta_{\nu}^{\beta}-\delta_{\nu}^{\alpha} \delta_{\mu}^{\beta}\right)\left\langle p_{A}, p_{B}\right|\left\{\left[\psi_{A}^{m}(x) \gamma_{\alpha} \Xi_{1}^{n}(0)\right]\left[\psi_{B}^{n}(0) \gamma_{\beta} \psi_{B}^{m}(x)\right]\right. \\
& +\left[\bar{\Xi}_{1}^{m}(x) \gamma_{\alpha} \psi_{A}^{n}(0)\right]\left[\psi_{B}^{n}(0) \gamma_{\beta} \psi_{B}^{m}(x)\right]+\left[\psi_{A}^{m}(x) \gamma_{\alpha} \psi_{A}^{n}(0)\right]\left[\psi_{B}^{n}(0) \gamma_{\beta} \Xi_{2}^{m}(x)\right] \\
& \left.+\left[\psi_{A}^{m}(x) \gamma_{\alpha} \psi_{A}^{n}(0)\right]\left[\bar{\Xi}_{2}^{n}(0) \gamma_{\beta} \psi_{B}^{m}(x)\right]+\gamma_{\alpha} \otimes \gamma_{\beta} \leftrightarrow \gamma_{\alpha} \gamma_{5} \otimes \gamma_{\beta} \gamma_{5}\right\}\left|p_{A}, p_{B}\right\rangle+x \leftrightarrow 0
\end{aligned}
$$

Let us consider the first term in the r.h.s. of the above equation. As demonstrated in ref. [16], the only non-negligible contribution comes from longitudinal $\mu$ and transverse $\nu$ (or vice versa), the term $\sim g_{\mu \nu}$ vanishes, and we obtain

$$
\begin{aligned}
\check{W}_{1 \mu \nu}^{(1) \mathrm{F}}(x)= & \frac{N_{c}}{2 s}\langle A, B|\left[\psi_{A}^{m}(x) \gamma_{\mu} \Xi_{1}^{n}(0)\right]\left[\psi_{B}^{n}(0) \gamma_{\nu} \psi_{B}^{m}(x)\right] \\
& +\gamma_{\mu} \otimes \gamma_{\nu} \leftrightarrow \gamma_{\mu} \gamma_{5} \otimes \gamma_{\nu} \gamma_{5}|A, B\rangle+\mu \leftrightarrow \nu+x \leftrightarrow 0 \\
= & \frac{p_{2 \mu}}{s^{3}}\left\{\left[\left\langle\bar{\psi}(x) \gamma_{\nu_{\perp}} \not p_{2} \gamma_{i} \frac{1}{\alpha} \psi(0)\right\rangle_{A}\left\langle\bar{\psi} B^{i}(0) \not p_{1} \psi(x)\right\rangle_{B}+\left\langle\bar{\psi}(x) \not p_{1} \not p_{2} \gamma_{i} \frac{1}{\alpha} \psi(0)\right\rangle_{A}\right.\right. \\
& \times\left\langle\bar{\psi} B^{i}(0) \gamma_{\nu_{\perp}} \psi(x)\right\rangle_{B}+\left(\psi(0) \otimes \psi(x) \leftrightarrow \gamma_{5} \psi(0) \otimes \gamma_{5} \psi(x)\right] \\
= & \frac{p_{2 \mu}}{s^{3}}\left[\left\langle\bar{\psi}\left(x_{\bullet}, x_{\perp}\right) \not p_{2} \frac{1}{\alpha} \psi(0)\right\rangle_{A}\left\langle\bar{\psi} \not B(0) \not p_{1} \gamma_{\nu}^{\perp} \psi\left(x_{\star}, x_{\perp}\right)\right\rangle_{B}\right. \\
& \left.+i\left\langle\bar{\psi}\left(x_{\bullet}, x_{\perp}\right) \sigma_{\star \nu} \frac{1}{\alpha} \psi(0)\right\rangle_{A}\left\langle\bar{\psi} \not B(0) \hat{p}_{1} \psi\left(x_{\star}, x_{\perp}\right)\right\rangle_{B}\right]+\mu \leftrightarrow \nu+x \leftrightarrow 0
\end{aligned}
$$

Here we separated color-singlet contributions and used eq. (A.21) to reduce number of $\gamma$-matrices.

Using formulas (A.45), (A.46), (A.48), and (A.51) for quark-antiquark-gluon operators and parametrizations from section A.2 we get the contribution to $W_{\mu \nu}$ in the form

$$
\begin{aligned}
W_{1 \mu \nu}^{(1) \mathrm{F}}(q)= & \frac{N_{c}}{16 \pi^{4}} \frac{1}{s} \int d x_{\bullet} d x_{\star} d^{2} x_{\perp} e^{-i \alpha x_{\bullet}-i \beta x_{\star}+i(q, x)_{\perp}} \\
& \times\left\langle A, B\left|\left[\bar{\psi}_{A}(x) \gamma_{\mu} \psi_{B}(x)\right]\left[\bar{\psi}_{B}(0) \gamma_{\nu} \Xi_{1}(0)\right]+x \leftrightarrow 0\right| A, B\right\rangle+\mu \leftrightarrow \nu \\
= & \frac{1}{64 \pi^{6}} \frac{p_{2 \mu}}{s^{3}} \int d^{2} k_{\perp} \int d x_{\bullet} d^{2} x_{\perp} e^{-i \alpha x_{\bullet}+i(k, x)_{\perp}} \int d x_{\star} d^{2} x_{\perp}^{\prime} e^{-i \beta x_{\star}+i\left(q-k, x^{\prime}\right)_{\perp}} \\
& \times\left[\left\langle\bar{\psi}\left(x_{\bullet}, x_{\perp}\right) \not p_{2} \frac{1}{\alpha} \psi(0)\right\rangle_{A}\left\langle\bar{\psi} \not B(0) \not p_{1} \gamma_{\nu}^{\perp} \psi\left(x_{\star}, x_{\perp}^{\prime}\right)\right\rangle_{B}+x \leftrightarrow 0\right]+\mu \leftrightarrow \nu \\
= & \frac{p_{2 \mu}}{\alpha_{q} s} \int d^{2} k_{\perp}(q-k)_{\nu} F^{f}\left(q, k_{\perp}\right)+\mu \leftrightarrow \nu
\end{aligned}
$$

where $f_{1} \leftrightarrow \bar{f}_{1}$ term in $F$ comes from $x \leftrightarrow 0$ contribution. 
As demonstrated in ref. [16], contribution of the second term in r.h.s. of eq. (4.13)

$$
\check{W}_{2 \mu \nu}^{(1) \mathrm{F}}(x)=\frac{N_{c}}{2 s}\langle A, B|\left[\bar{\Xi}_{1}^{m}(x) \gamma_{\mu} \psi_{A}^{n}(0)\right]\left[\psi_{B}^{n}(0) \gamma_{\nu} \psi_{B}^{m}(x)\right]+\ldots
$$

doubles the result (4.15) and the result for the third and the fourth terms is obtained from eq. (4.15) by replacement $\frac{p_{2 \mu}(q-k)_{\nu}^{\perp}}{\alpha_{q} s} \leftrightarrow \frac{p_{1 \mu} k_{\nu}^{\perp}}{\beta_{q} s}$ so

$$
W_{\mu \nu}^{(1) \mathrm{F}}(q)=2 \int d^{2} k_{\perp}\left(\frac{p_{1 \mu} k_{\nu}^{\perp}}{\beta_{q} s}+\frac{p_{2 \mu}(q-k)_{\nu}^{\perp}}{\alpha_{q} s}\right) F^{f}\left(q, k_{\perp}\right)+\mu \leftrightarrow \nu
$$

This result agrees with the corresponding $1 / Q$ terms in ref. [20].

\subsubsection{Terms with two quark-quark-gluon TMDs (two-gluon terms)}

Let us now consider terms in $W_{\mu \nu}^{F}$ from eq. (4.6) with two gluon operators. The first of such terms is

$$
\begin{aligned}
\check{W}_{1 \mu \nu}^{(2 \mathrm{a}) \mathrm{F}}(x)= & \frac{N_{c}}{2 s}\left(g_{\mu \nu} g^{\alpha \beta}-\delta_{\mu}^{\alpha} \delta_{\nu}^{\beta}-\delta_{\nu}^{\alpha} \delta_{\mu}^{\beta}\right)\left\langle p_{A}, p_{B}\right|\left\{\left[\psi_{A}^{m}(x) \gamma_{\alpha} \Xi_{1}^{n}(0)\right]\left[\psi_{B}^{n}(0) \gamma_{\beta} \Xi_{2}^{m}(x)\right]\right. \\
& \left.+\left[\Xi_{1}^{m}(x) \gamma_{\alpha} \psi_{A}^{n}(0)\right]\left[\Xi_{2}^{n}(0) \gamma_{\beta} \psi_{B}^{m}(x)\right]+\gamma_{\alpha} \otimes \gamma_{\beta} \leftrightarrow \gamma_{\alpha} \gamma_{5} \otimes \gamma_{\beta} \gamma_{5}\right\}\left|p_{A}, p_{B}\right\rangle+x \leftrightarrow 0
\end{aligned}
$$

It is convenient to start from the contribution

$$
\begin{aligned}
\check{V}_{\mu \nu}^{1 \mathrm{~F}}(x)= & \frac{N_{c}}{2 s}\langle A, B|\left\{\left[\psi_{A}^{m}(x) \gamma_{\mu} \Xi_{1}^{n}(0)\right]\left[\psi_{B}^{n}(0) \gamma_{\nu} \Xi_{2}^{m}(x)\right]\right. \\
& \left.+\gamma_{\mu} \otimes \gamma_{\nu} \leftrightarrow \gamma_{\mu} \gamma_{5} \otimes \gamma_{\nu} \gamma_{5}\right\}|A, B\rangle+\mu \leftrightarrow \nu+x \leftrightarrow 0 \\
= & -\frac{1}{2 s^{3}}\left\{\left\langle\bar{\psi} A_{i}(x) \gamma_{\mu} \not_{2} \gamma^{j} \frac{1}{\alpha} \psi(0)\right\rangle_{A}\left\langle\bar{\psi} B_{j}(0) \gamma_{\nu} \not p_{1} \gamma^{i} \frac{1}{\beta} \psi(x)\right\rangle_{B}\right. \\
& \left.+\psi(0) \otimes \psi(x) \leftrightarrow \gamma_{5} \psi(0) \otimes \gamma_{5} \psi(x)+\mu \leftrightarrow \nu\right\}+x \leftrightarrow 0
\end{aligned}
$$

As demonstrated in ref. [16], the non-negligible contribution comes only from transverse $\mu$ and $\nu$. In this case we can use formula (A.27) and get

$$
\begin{aligned}
\check{V}_{\mu_{\perp} \nu_{\perp}}^{1 \mathrm{~F}}= & -\frac{1}{2 s^{3}}\left\{\left\langle\bar{\psi} A_{i}(x) \gamma_{\mu_{\perp}} \not p_{2} \gamma^{j} \frac{1}{\alpha} \psi(0)\right\rangle_{A}\left\langle\bar{\psi} B_{j}(0) \gamma_{\nu_{\perp}} \not p_{1} \gamma^{i} \frac{1}{\beta} \psi(x)\right\rangle_{B}\right. \\
& \left.+\psi(0) \otimes \psi(x) \leftrightarrow \gamma_{5} \psi(0) \otimes \gamma_{5} \psi(x)+\mu \leftrightarrow \nu\right\}+x \leftrightarrow 0 \\
= & -\frac{g_{\mu \nu}^{\perp}}{s^{3}}\left\langle\bar{\psi} \not A(x) \not p_{2} \gamma_{i} \frac{1}{\alpha} \psi(0)\right\rangle_{A}\left\langle\bar{\psi} \not B(0) \not p_{1} \gamma^{i} \frac{1}{\beta} \psi(x)\right\rangle_{B}+x \leftrightarrow 0
\end{aligned}
$$

This gives the contribution of the first matrix element in the r.h.s. of eq. (4.18) to $W_{\mu \nu}(q)$ in the form

$$
\begin{aligned}
& \frac{1}{2}\left(g_{\mu \nu} g^{\alpha \beta}-\delta_{\mu}^{\alpha} \delta_{\nu}^{\beta}-\delta_{\nu}^{\alpha} \delta_{\mu}^{\beta}\right) \frac{1}{16 \pi^{4}} \int d x_{\bullet} d x_{\star} d^{2} x_{\perp} e^{-i \alpha_{q} x_{\bullet}-i \beta_{q} x_{\star}+i(q, x)_{\perp}} \check{V}_{2 \mu_{\perp} \nu_{\perp}}(x) \\
& \quad=\frac{g_{\mu \nu}^{\|}}{Q_{\|}^{2}} \int d^{2} k_{\perp}(k, q-k)_{\perp} F^{f}\left(q, k_{\perp}\right)
\end{aligned}
$$


where we again used formulas from appendices A.2 and A.3. Next, as shown in ref. [16], the contribution of the second matrix element in the r.h.s. of eq. (4.18) is equal to that of the first one so we get

$$
W_{\mu \nu}^{(2 \mathrm{a}) \mathrm{F}}(q)=\frac{2 g_{\mu \nu}^{\|}}{Q_{\|}^{2}} \int d^{2} k_{\perp}(k, q-k)_{\perp} F^{f}\left(q, k_{\perp}\right)
$$

The second two-gluon contribution to $\check{W}^{F}$ in eq. (4.6) is

$$
\begin{aligned}
\check{W}_{\mu \nu}^{(2 \mathrm{~b}) \mathrm{F}}(x)= & -\frac{N_{c}}{2 s}\left(\delta_{\mu}^{\alpha} \delta_{\nu}^{\beta}+\delta_{\nu}^{\alpha} \delta_{\mu}^{\beta}-g_{\mu \nu} g^{\alpha \beta}\right)\langle A, B|\left\{\left[\bar{\psi}_{A}^{m}(x) \gamma_{\alpha} \psi_{A}^{n}(0)\right]\left[\bar{\Xi}_{2}^{n}(0) \gamma_{\beta} \Xi_{2}^{m}(x)\right]\right. \\
& \left.+\left[\bar{\Xi}_{1}^{m}(x) \gamma_{\alpha} \Xi_{1}^{n}(0)\right]\left[\bar{\psi}_{B}^{n}(0) \gamma_{\beta} \psi_{B}^{m}(x)\right]+\gamma_{\alpha} \otimes \gamma_{\beta} \leftrightarrow \gamma_{\alpha} \gamma_{5} \otimes \gamma_{\beta} \gamma_{5}\right\}|A, B\rangle+x \leftrightarrow 0
\end{aligned}
$$

Let us consider the first matrix element in the r.h.s. of the above equation. We get

$$
\begin{aligned}
\check{W}_{1 \mu \nu}^{(2 \mathrm{~b}) \mathrm{F}}(x)= & -\frac{N_{c}}{2 s}\left(\delta_{\mu}^{\alpha} \delta_{\nu}^{\beta}+\delta_{\nu}^{\alpha} \delta_{\mu}^{\beta}-g_{\mu \nu} g^{\alpha \beta}\right) \\
& \times\langle A, B|\left\{\left[\bar{\psi}_{A}^{m}(x) \gamma_{\alpha} \psi_{A}^{n}(0)\right]\left[\bar{\Xi}_{2}^{n}(0) \gamma_{\beta} \Xi_{2}^{m}(x)\right]\left|p_{A}, p_{B}\right\rangle+x \leftrightarrow 0\right. \\
= & -\frac{1}{s^{3}}\left(\delta_{\mu}^{\alpha} p_{1 \nu}+\delta_{\nu}^{\alpha} p_{1 \mu}-g_{\mu \nu} p_{1}^{\alpha}\right)\left(\left\langle\bar{\psi}(x) A_{j}(x) \gamma_{\alpha} A_{i}(0) \psi(0)\right\rangle_{A}\right. \\
& \left.\times\left\langle\left(\bar{\psi} \frac{1}{\beta}\right)(0) \gamma^{i} \not p_{1} \gamma^{j} \frac{1}{\beta} \psi(x)\right\rangle_{B}+\psi(0) \otimes \psi(x) \leftrightarrow \gamma_{5} \psi(0) \otimes \gamma_{5} \psi(x)\right)+x \leftrightarrow 0 \\
= & -\frac{4 p_{1 \mu} p_{1 \nu}}{s^{4}}\left(\left\langle\bar{\psi}(x) A_{j}(x) \not p_{2} A_{i}(0) \psi(0)\right\rangle_{A}\left\langle\left(\bar{\psi} \frac{1}{\beta}\right)(0) \gamma^{i} p_{1} \gamma^{j} \frac{1}{\beta} \psi(x)\right\rangle_{B}\right. \\
& \left.+\psi(0) \otimes \psi(x) \leftrightarrow \gamma_{5} \psi(0) \otimes \gamma_{5} \psi(x)\right)
\end{aligned}
$$

up to the terms which are negligible as shown in ref. [16]. Using eq. (A.15) this can be rewritten as

$$
\begin{aligned}
\check{W}_{1 \mu \nu}^{(2 \mathrm{~b}) \mathrm{F}}(x)= & -\frac{4 p_{1 \mu} p_{1 \nu}}{s^{4}}\left(\left\langle\bar{\psi}(x) \not A(x) \not p_{2} \not A(0) \psi(0)\right\rangle_{A}\left\langle\left(\bar{\psi} \frac{1}{\beta}\right)(0) \not p_{1} \frac{1}{\beta} \psi(x)\right\rangle_{B}\right. \\
& \left.+\psi(0) \otimes \psi(x) \leftrightarrow \gamma_{5} \psi(0) \otimes \gamma_{5} \psi(x)\right)+x \leftrightarrow 0
\end{aligned}
$$

The corresponding contribution to $W_{\mu \nu}(q)$ is obtained from QCD equation of motion (A.60) and formula (A.46) from appendix A.3:

$$
W_{1 \mu \nu}^{(2 \mathrm{~b}) \mathrm{F}}(q)=\frac{4 p_{1 \mu} p_{1 \nu}}{\beta_{q}^{2} s^{2}} \int d^{2} k_{\perp} k_{\perp}^{2} F^{f}\left(q, k_{\perp}\right)
$$

Next, as shown in ref. [16], the contribution of the second matrix element in the r.h.s. of the eq. (4.27)

$$
\begin{aligned}
\check{W}_{2 \mu \nu}^{(2 \mathrm{~b}) \mathrm{F}}(x)= & -\frac{N_{c}}{2 s}\left(\delta_{\mu}^{\alpha} \delta_{\nu}^{\beta}+\delta_{\nu}^{\alpha} \delta_{\mu}^{\beta}-g_{\mu \nu} g^{\alpha \beta}\right) \\
& \left.\langle A, B|\left[\bar{\Xi}_{1}^{m}(x) \gamma_{\alpha} \Xi_{1}^{n}(0)\right]\left[\bar{\psi}_{B}^{n}(0) \gamma_{\beta} \psi_{B}^{m}(x)\right]+\gamma_{\alpha} \otimes \gamma_{\beta} \leftrightarrow \gamma_{\alpha} \gamma_{5} \otimes \gamma_{\beta} \gamma_{5}\right\}|A, B\rangle+x \leftrightarrow 0
\end{aligned}
$$


differs from eq. (4.26) by replacements $p_{1} \leftrightarrow p_{2}, \alpha_{q} \leftrightarrow \beta_{q}$ and exchange of projectile matrix elements and the target ones so we finally get

$$
W_{\mu \nu}^{(2 \mathrm{~b}) \mathrm{F}}(q)=\int d^{2} k_{\perp}\left[\frac{4 p_{1 \mu} p_{1 \nu}}{\beta_{q}^{2} s^{2}} k_{\perp}^{2}+\frac{4 p_{2 \mu} p_{2 \nu}}{\alpha_{q}^{2} s^{2}}(q-k)_{\perp}^{2}\right] F^{f}\left(q, k_{\perp}\right)
$$

The third two-gluon contribution to $\check{W}^{F}$ in eq. (4.6) has the form

$$
\begin{aligned}
\check{W}_{\mu \nu}^{(2 \mathrm{c}) \mathrm{F}}(x)= & \frac{N_{c}}{2 s}\left(g_{\mu \nu} g^{\alpha \beta}-\delta_{\mu}^{\alpha} \delta_{\nu}^{\beta}-\delta_{\nu}^{\alpha} \delta_{\mu}^{\beta}\right)\left\langle p_{A}, p_{B}\right|\left\{\left[\bar{\Xi}_{1}^{m}(x) \gamma_{\alpha} \psi_{A}^{n}(0)\right]\left[\psi_{B}^{n}(0) \gamma_{\beta} \Xi_{2}^{m}(x)\right]\right. \\
& \left.+\left[\psi_{A}^{m}(x) \gamma_{\alpha} \Xi_{1}^{n}(0)\right]\left[\Xi_{2}^{n}(0) \gamma_{\beta} \psi_{B}^{m}(x)\right]+\gamma_{\alpha} \otimes \gamma_{\beta} \leftrightarrow \gamma_{\alpha} \gamma_{5} \otimes \gamma_{\beta} \gamma_{5}\right\}\left|p_{A}, p_{B}\right\rangle+x \leftrightarrow 0
\end{aligned}
$$

It is easy to see that after separating color-singlet matrix elements this term is $O\left(\frac{1}{N_{c}^{2}}\right)$ in comparison to (4.19) so we neglect it.

Thus, the result for $W_{\mu \nu}^{\mathrm{F}}(q)$ is the sum of eqs. (4.11), (4.17), (4.22), and (4.28). After some algebra, it can be rewritten as

$$
W_{\mu \nu}^{\mathrm{F}}(q)=\sum_{f} e_{f}^{2} W_{\mu \nu}^{\mathrm{fF}}(q), \quad W_{\mu \nu}^{\mathrm{fF}}(q)=\int d^{2} k_{\perp} F^{f}\left(q, k_{\perp}\right) \mathcal{W}_{\mu \nu}^{F}\left(q, k_{\perp}\right)
$$

where

$$
\begin{aligned}
\mathcal{W}_{\mu \nu}^{\mathrm{F}}\left(q, k_{\perp}\right)= & -g_{\mu \nu}^{\perp}+\frac{1}{Q_{\|}^{2}}\left(q_{\mu}^{\|} q_{\nu}^{\perp}+q_{\nu}^{\|} q_{\mu}^{\perp}\right)+\frac{q_{\perp}^{2}}{Q_{\|}^{4}} q_{\mu}^{\|} q_{\nu}^{\|}+\frac{\tilde{q}_{\mu} \tilde{q}_{\nu}}{Q_{\|}^{2}}\left[q_{\perp}^{2}-4(k, q-k)_{\perp}\right] \\
& -\left[\frac{\tilde{q}_{\mu}}{Q_{\|}^{2}}\left(g_{\nu i}^{\perp}-\frac{q_{\nu}^{\|} q_{i}}{Q_{\|}^{2}}\right)(q-2 k)_{\perp}^{i}+\mu \leftrightarrow \nu\right]
\end{aligned}
$$

It is easy to see that $q^{\mu} \mathcal{W}_{\mu \nu}^{\mathrm{F}}\left(q, k_{\perp}\right)=0$.

\section{2 $W^{\mathrm{G}}$ term of eq. (4.8)}

In this section we will repeat the above calculations for the $W_{\mu \nu}^{\mathrm{G}}(q)$. Let us start from

$$
\begin{aligned}
\check{W}_{\mu \nu}^{\mathrm{G}}(x)= & -\frac{N_{c} g_{\mu \nu}}{2 s}\langle A, B|\left[\bar{\Psi}_{1}^{m}(x) \Psi_{1}^{n}(0)\right]\left[\bar{\Psi}_{2}^{n}(0) \Psi_{2}^{m}(x)\right] \\
& -\left[\bar{\Psi}_{1}^{m}(x) \gamma_{5} \Psi_{1}^{n}(0)\right]\left[\bar{\Psi}_{2}^{n}(0) \gamma_{5} \Psi_{2}^{m}(x)\right]|A, B\rangle+x \leftrightarrow 0
\end{aligned}
$$

First, as seen from parametrizations (A.39) and (A.40), the leading-twist contribution can be neglected. Second, as demonstrated in ref. [16], the contribution of one-gluon operators 
also vanishes with our accuracy. Let us now consider two-gluon terms and start with

$$
\begin{aligned}
\check{W}_{\mu \nu}^{\mathrm{G}}(x)= & -\frac{N_{c} g_{\mu \nu}}{2 s}\langle A, B|\left[\bar{\psi}_{A}^{n}(x) \Xi_{1}^{m}(0)\right]\left[\bar{\psi}_{B}^{n}(0) \Xi_{2}^{m}(x)\right] \\
& -\left[\bar{\psi}_{A}^{m}(x) \gamma_{5} \Xi_{1}^{n}(0)\right]\left[\bar{\psi}_{B}^{n}(0) \gamma_{5} \Xi_{2}^{m}(x)\right]|A, B\rangle+x \leftrightarrow 0 \\
= & \frac{g_{\mu \nu}}{2 s^{3}}\left[\left\langle\bar{\psi} A^{i}(x) \sigma_{\star j} \frac{1}{\alpha} \psi(0)\right\rangle_{A}\left\langle\bar{\psi} B^{j}(0) \sigma_{\bullet i} \frac{1}{\beta} \psi(x)\right\rangle_{B}\right. \\
& \left.-\psi(0) \otimes \psi(x) \leftrightarrow \gamma_{5} \psi(0) \otimes \gamma_{5} \psi(x)\right]+x \leftrightarrow 0 \\
= & -\frac{g_{\mu \nu}}{2 s^{3}}\left\langle\bar{\psi} A(x) \not p_{2} \frac{1}{\alpha} \psi(0)\right\rangle_{A}\left\langle\bar{\psi} \not B(0) \not p_{1} \frac{1}{\beta} \psi(x)\right\rangle_{B}\left[1+O\left(\frac{q_{\perp}^{2}}{s}\right)\right]
\end{aligned}
$$

where we used formula (A.35) and the fact that ${ }^{6}$

$$
\left\langle\bar{\psi}(x)\left[A_{k} \sigma_{\star j}-A_{j}(x) \sigma_{\star k}\right] \psi(0)\right\rangle_{A}=0
$$

It is easy to see that with our accuracy the above equation is the only two-gluon contribution to $\check{W}_{\mu \nu}^{\mathrm{G}}(x)$ since $\bar{\Xi}_{1} \Xi_{1}=\bar{\Xi}_{2} \Xi_{2}=0$ and the matrix element $\left\langle\left[\bar{\Xi}_{1}^{m}(x) \gamma_{\alpha} \psi_{A}^{n}(0)\right]\left[\psi_{B}^{n}(0) \gamma_{\beta} \Xi_{2}^{m}(x)\right]\right\rangle$ is $\sim O\left(\frac{1}{N_{c}^{2}}\right)$ in comparison to eq. (4.34) similarly to eq. (4.29).

Now, using equations (A.45), (A.56), (A.58) and parametrizations (A.44) we obtain the contribution to photon-mediated hadronic tensor in the form

$$
\begin{aligned}
W_{\mu \nu}^{\mathrm{G}}(q) & =\frac{g_{\mu \nu}}{16 \pi^{4}} \int d x_{\bullet} d x_{\star} d^{2} x_{\perp} e^{-i \alpha_{q} x_{\bullet}-i \beta_{q} x_{\star}+i(q, x)_{\perp}} \check{W}_{\mu \nu}^{\mathrm{G}}(x) \\
& =-\frac{g_{\mu \nu}}{2 Q_{\|}^{2}} \int d^{2} k_{\perp} \frac{1}{m^{2}} k_{\perp}^{2}(q-k)_{\perp}^{2} H\left(q, k_{\perp}\right)
\end{aligned}
$$

where

$$
H^{f}\left(q, k_{\perp}\right)=h_{1}^{\perp f}\left(\alpha_{q}, k_{\perp}\right) \bar{h}_{1}^{\perp f}\left(\beta_{q},(q-k)_{\perp}\right)+h_{1}^{\perp f} \leftrightarrow \bar{h}_{1}^{\perp f}
$$

for the flavor that we are considering.

\section{3 $W^{\mathrm{T}}$ contribution of eq. (4.9)}

In this section we calculate the $W_{\mu \nu}^{\mathrm{T}}$ term of eq. (4.9). The leading-twist contribution

$$
\begin{aligned}
\check{W}_{\mu \nu}^{\mathrm{T}, \mathrm{lt}}(x) & =\check{W}_{\mu \nu}^{\mathrm{H}, \mathrm{lt}}(x) \\
& \left.=\frac{N_{c}}{2 s}\left(\delta_{\mu}^{\alpha} \delta_{\nu}^{\beta}+\delta_{\nu}^{\alpha} \delta_{\mu}^{\beta}-\frac{1}{2} g_{\mu \nu} g^{\alpha \beta}\right)\langle A, B|\left[\psi_{A}^{m}(x) \sigma_{\alpha \xi} \psi_{A}^{n}(0)\right] \psi_{B}^{n}(0) \sigma_{\beta}^{\xi} \psi_{B}^{m}(x)\right]|A, B\rangle+x \leftrightarrow 0
\end{aligned}
$$

is easily obtained from parametrizations (A.44) [21]:

$$
\begin{aligned}
& W_{\mu \nu}^{\mathrm{H}, \mathrm{lt}}\left(\alpha_{q}, \beta_{q}, q_{\perp}\right)=\frac{1}{16 \pi^{4}} \int d x_{\bullet} d x_{\star} d^{2} x_{\perp} e^{-i \alpha_{q} x_{\bullet}-i \beta_{q} x_{\star}+i(q, x)_{\perp}} \check{W}_{\mu \nu}^{\mathrm{lt}}(x) \\
& \quad=-\sum_{f} e_{f}^{2} \int d^{2} k_{\perp}\left[k_{\mu}^{\perp}(q-k)_{\nu}^{\perp}+k_{\nu}^{\perp}(q-k)_{\mu}^{\perp}+g_{\mu \nu}^{\perp}(k, q-k)_{\perp}\right] H^{f}\left(q, k_{\perp}\right)
\end{aligned}
$$

\footnotetext{
${ }^{6} \mathrm{~A}$ rigorous argument goes like that: the matrix element (4.34) can be rewritten as $\epsilon_{\nu_{\perp} j} \epsilon_{k l}\left\langle\bar{\psi}(0)\left[A_{k}(0) \sigma_{\bullet} \psi \psi(x)\right\rangle=\epsilon_{j \nu_{\perp}}\left\langle\bar{\psi}(0) \not A(0) \not p_{1} \gamma_{5} \psi(x)\right\rangle\right.$. As demonstrated in section A.3, $\not A$ in this formula can be replaced by $\not k_{\perp}$ so the contribution is proportional to matrix element $k^{i}\left\langle\bar{\psi}(0) i \sigma_{\bullet} \gamma_{5} \psi(x)\right\rangle=$ $k^{i} \epsilon_{i j}\left\langle\bar{\psi}(0) \sigma_{\bullet} \psi(x)\right\rangle$ which vanishes as seen from the parametrization (A.44). .
} 
For the calculation of one- and two-gluon terms it is convenient to consider

$$
\check{V}_{\mu \nu}^{\mathrm{H}}(x)=\frac{N_{c}}{2 s}\langle A, B|\left[\bar{\Psi}_{1}^{m}(x) \sigma_{\mu \xi} \Psi_{1}^{n}(0)\right]\left[\bar{\Psi}_{2}^{n}(0) \sigma_{\nu}{ }^{\xi} \Psi_{2}^{m}(x)|A, B\rangle+\mu \leftrightarrow \nu+x \leftrightarrow 0\right.
$$

and subtract trace to get $\check{W}_{\mu \nu}^{\mathrm{T}}(x)$ afterwards.

\subsubsection{One-gluon terms in $\check{V}_{\mu \nu}^{\mathrm{H}}(x)$}

Let us start from one-gluon term coming from $\Xi_{2}$. Sorting out color-singlet matrix elements, we get

$$
\check{V}_{1 \mu \nu}^{(1) \mathrm{H}}(x)=-\frac{1}{2 s^{2}}\left\langle\bar{\psi}(x) \sigma_{\mu \xi} \not p_{2} \gamma^{i} \frac{1}{\alpha} \psi(0)\right\rangle_{A}\left\langle\bar{\psi} B^{i}(0) \sigma_{\nu}^{\xi} \psi(x)\right\rangle_{B}+\mu \leftrightarrow \nu+x \leftrightarrow 0
$$

As demonstrated in ref. [16], the only non-negligible contributions are those with one of the indices in eq. (4.40) longitudinal and one transverse. For example, let $\mu$ be longitudinal and $\nu$ transverse, the opposite case will differ by replacement $\mu \leftrightarrow \nu$. Using the decomposition of $g^{\mu \nu}$ in longitudinal and transverse part (3.1) we get

$$
\begin{aligned}
& \left(\frac{2 p_{1}^{\mu} p_{2}^{\mu^{\prime}}}{s}+\mu \leftrightarrow \mu^{\prime}\right) \check{V}_{1 \mu \nu_{\perp}}^{(1) \mathrm{H}}(x)=-\left(\frac{p_{2 \mu} p_{1}^{\mu^{\prime}}}{s^{3}}+\mu \leftrightarrow \mu^{\prime}\right) \\
& \quad \times\left[\left\langle\bar{\psi}(x) \sigma_{\mu^{\prime} \xi} \not p_{2} \gamma^{i} \frac{1}{\alpha} \psi(0)\right\rangle_{A}\left\langle\bar{\psi} B^{i}(0) \sigma_{\nu_{\perp}}^{\xi} \psi(x)\right\rangle_{B}+\mu^{\prime} \leftrightarrow \nu\right]+x \leftrightarrow 0
\end{aligned}
$$

As demonstrated in ref. [16], the term proportional to $p_{1 \mu}$ is small so we get

$$
\begin{aligned}
& \check{V}_{1 \mu_{\|} \nu_{\perp}}^{(1) \mathrm{H}}(x)= \\
& \quad \frac{p_{2 \mu}}{s^{3}}\left\{i\left\langle\bar{\psi}(x) \sigma_{\nu_{\perp} j} \sigma_{\star i} \frac{1}{\alpha} \psi(0)\right\rangle_{A}\left\langle\bar{\psi} B^{i}(0) \sigma_{\bullet}^{j} \psi(x)\right\rangle_{B}-\left\langle\bar{\psi}(x) \sigma_{\star i} \frac{1}{\alpha} \psi(0)\right\rangle_{A}\left\langle\bar{\psi} B^{i}(0) \sigma_{\bullet \nu_{\perp}} \psi(x)\right\rangle_{B}\right. \\
& \quad+i\left\langle\bar{\psi}(x) \sigma_{\bullet j} \sigma_{\star i} \frac{1}{\alpha} \psi(0)\right\rangle_{A}\left\langle\bar{\psi} B^{i}(0) \sigma_{\nu_{\perp}}^{j} \psi(x)\right\rangle_{B} \\
& \left.\quad+\frac{2 i}{s}\left\langle\bar{\psi}(x) \sigma_{\bullet \nu_{\perp}} \sigma_{\star i} \frac{1}{\alpha} \psi(0)\right\rangle_{A}\left\langle\bar{\psi} B^{i}(0) \sigma_{\star \bullet} \psi(x)\right\rangle_{B}\right\}+x \leftrightarrow 0
\end{aligned}
$$

Using eq. (A.9) one can prove that the contribution of last two terms in the r.h.s. is $\sim \frac{q_{\perp}^{2}}{s}$ and therefore

$$
\begin{aligned}
\check{V}_{1 \mu_{\|} \nu_{\perp}}^{(1) \mathrm{H}}(x)= & \frac{p_{2 \mu}}{s^{3}}\left[i\left\langle\bar{\psi}(x) \sigma_{\nu_{\perp} j} \sigma_{\star i} \frac{1}{\alpha} \psi(0)\right\rangle_{A}\left\langle\bar{\psi} B^{i}(0) \sigma_{\bullet}^{j} \psi(x)\right\rangle_{B}\right. \\
& \left.-\left\langle\bar{\psi}(x) \sigma_{\star i} \frac{1}{\alpha} \psi(0)\right\rangle_{A}\left\langle\bar{\psi} B^{i}(0) \sigma_{\bullet \nu_{\perp}} \psi(x)\right\rangle_{B}\right]+\leftrightarrow 0 \\
= & \frac{p_{2 \mu}}{s^{3}}\left\langle\bar{\psi}(x) \sigma_{\star}{ }^{j} \frac{1}{\alpha} \psi(0)\right\rangle_{A}\left\langle\bar{\psi}(0)\left[B_{\nu}(0) \sigma_{\bullet j}-\nu \leftrightarrow j\right] \psi(x)\right\rangle_{B}-\frac{p_{2 \mu}}{s^{3}}\left\langle\bar{\psi}(x) \sigma_{\star \nu_{\perp}} \frac{1}{\alpha} \psi(0)\right\rangle_{A} \\
& \times\left\langle\bar{\psi} B^{j}(0) \sigma_{\bullet j} \psi(x)\right\rangle_{B}+x \leftrightarrow 0=\frac{i p_{2 \mu}}{s^{3}}\left\langle\bar{\psi}(x) \sigma_{\star \nu_{\perp}} \frac{1}{\alpha} \psi(0)\right\rangle_{A}\left\langle\bar{\psi}(0) \not B(0) \not p_{1} \psi(x)\right\rangle_{B}+x \leftrightarrow 0
\end{aligned}
$$

where we used formulas (4.34) and (A.9). 
Using formulas (A.45), (A.46), (A.56), and (A.58) for quark-antiquark-gluon operators and parametrizations from section A.2 we get the contribution to $W_{\mu \nu}$ in the form

$$
\begin{aligned}
V_{1 \mu \nu}^{(1) \mathrm{H}}(q) & =\frac{N_{c}}{16 \pi^{4}} \frac{1}{s} \int d x_{\bullet} d x_{\star} d^{2} x_{\perp} e^{-i \alpha x_{\bullet}-i \beta x_{\star}+i(q, x)_{\perp}} \check{V}_{1 \mu \nu}^{(1) H}(x) \\
& =-\frac{p_{2 \mu}}{\alpha_{q} s} \int d^{2} k_{\perp} k_{\nu} \frac{(q-k)_{\perp}^{2}}{m^{2}} H\left(q, k_{\perp}\right)+\mu \leftrightarrow \nu
\end{aligned}
$$

where terms with replacement $h_{1 f}^{\perp} \leftrightarrow \bar{h}_{1 f}^{\perp}$ come from $x \leftrightarrow 0$ contribution as usually.

Next, as proved in ref. [16], the term with $\bar{\Xi}_{1}(x)$ doubles the contribution (4.44) and the terms with $\Xi_{2}(0)$ and $\bar{\Xi}_{2}(x)$ are obtained by the projectile $\leftrightarrow$ target replacement, namely $p_{1} \leftrightarrow p_{2}, \alpha_{q} \leftrightarrow \beta_{q}$ and $k_{\perp} \leftrightarrow(q-k)_{\perp}$. Thus, the contribution of one-gluon terms to $W_{\mu \nu}^{\mathrm{T}}(q)$ has the form

$$
W_{\mu \nu}^{(1) \mathrm{T}}(q)=-2 \int d^{2} k_{\perp}\left[\frac{p_{1 \mu}(q-k)_{\nu}}{\beta_{q} s} \frac{k_{\perp}^{2}}{m^{2}}+\frac{p_{2 \mu} k_{\nu}}{\alpha_{q} s} \frac{(q-k)_{\perp}^{2}}{m^{2}}\right] H\left(q, k_{\perp}\right)+\mu \leftrightarrow \nu
$$

\subsubsection{Two-gluon terms in $\check{V}_{\mu \nu}^{\mathrm{H}}(x)$}

Let us start with the term

$$
\check{V}_{1 \mu \nu}^{(2 \mathrm{a}) \mathrm{H}}(x)=\frac{N_{c}}{2 s}\langle A, B|\left[\psi_{A}^{m}(x) \sigma_{\mu \xi} \Xi_{1}^{n}(0)\right]\left[\psi_{B}^{n}(0) \sigma_{\nu}^{\xi} \Xi_{2}^{m}(x)|A, B\rangle+\mu \leftrightarrow \nu+x \leftrightarrow 0\right.
$$

Separating color-singlet contributions, we get

$$
\check{V}_{1 \mu \nu}^{(2 \mathrm{a}) \mathrm{H}}(x)=\frac{1}{2 s^{3}}\left\langle\bar{\psi} A_{i}(x) \sigma_{\mu \alpha} \not \not_{2} \gamma^{j} \frac{1}{\alpha} \psi(0)\right\rangle_{A}\left\langle\bar{\psi} B_{j}(0) \sigma_{\nu}{ }^{\alpha} \not \not_{1} \gamma^{i} \frac{1}{\beta} \psi(x)\right\rangle_{B}+\mu \leftrightarrow \nu+x \leftrightarrow 0
$$

As demonstrated in ref. [16], the contributions from longitudinal $\mu$ and transverse $\nu$ (or vice versa) are small. For transverse $\mu$ and $\nu$ we obtain

$$
\begin{aligned}
\check{V}_{1 \mu_{\perp} \nu_{\perp}}^{(2 \mathrm{a}) \mathrm{H}}(x)= & \left.\left.-\frac{1}{2 s^{3}}\left\langle\bar{\psi} A^{i}(x) \sigma_{\mu_{\perp} k} \sigma_{\star j} \frac{1}{\alpha} \psi\right) 0\right)\right\rangle_{A}\left\langle\bar{\psi} B^{j}(0) \sigma_{\nu_{\perp}}^{k} \sigma_{\bullet} i \frac{1}{\beta} \psi(x)\right\rangle_{B} \\
& -\frac{1}{s^{4}}\left\langle\bar{\psi} A^{i}(x) \sigma_{\bullet \mu_{\perp}} \sigma_{\star j} \frac{1}{\alpha} \psi(0)\right\rangle_{A}\left\langle\bar{\psi} B^{j}(0) \sigma_{\star \nu_{\perp}} \sigma_{\bullet} i \frac{1}{\beta} \psi(x)\right\rangle_{B}+\mu \leftrightarrow \nu+x \leftrightarrow 0
\end{aligned}
$$

Using eq. (A.9) and (4.34), it is possible to demonstrate the second term in the r.h.s. is small and the first term can be rewritten as

$$
\begin{aligned}
\check{V}_{1 \mu_{\perp} \nu_{\perp}}^{(2 \mathrm{a}) \mathrm{H}}(x)= & -\frac{g_{\mu \nu}^{\perp}}{2 s^{3}}\left\langle\bar{\psi} A^{i}(x) \sigma_{\star i} \frac{1}{\alpha} \psi(0)\right\rangle_{A}\left\langle\bar{\psi} B^{j}(0) \sigma_{\bullet j} \frac{1}{\beta} \psi(x)\right\rangle_{B} \\
& +\frac{1}{s^{3}}\left\{\left\langle\bar{\psi}\left(A_{k} \sigma_{\star \mu_{\perp}}-\frac{1}{2} g_{\mu k} \sigma_{\star j} A^{j}\right)(x) \frac{1}{\alpha} \psi(0)\right\rangle_{A}\right. \\
& \left.\times\left\langle\bar{\psi}\left(B^{k} \sigma_{\bullet \nu_{\perp}}-\frac{1}{2} \delta_{\nu}^{k} \sigma_{\bullet j} B^{j}\right)(0) \frac{1}{\beta} \psi(x)\right\rangle_{B}+\mu \leftrightarrow \nu\right\}+x \leftrightarrow 0
\end{aligned}
$$


The corresponding contribution to $\check{V}_{\mu_{\perp}}^{\mathrm{H}}(q)$ can be obtained from QCD equations of motion (A.56), (A.58) and parametrization (A.63). We get

$$
\begin{aligned}
\check{V}_{1 \mu_{\perp} \nu_{\perp}}^{(2 \mathrm{a}) \mathrm{H}}(q)= & \frac{1}{Q_{\|}^{2} N_{c}} \int d^{2} k_{\perp}\left[g_{\mu \nu}^{\perp} \frac{k_{\perp}^{2}(q-k)_{\perp}^{2}}{2 m^{2}} H^{f}\left(q, k_{\perp}\right)\right. \\
& +\left[\left(k_{\mu}^{\perp}(q-k)_{\nu}^{\perp}+\mu \leftrightarrow \nu\right)(k, q-k)_{\perp}-k_{\perp}^{2}(q-k)_{\mu}^{\perp}(q-k)_{\nu}^{\perp}\right. \\
& \left.\left.-\left(q-k_{\perp}\right)^{2} k_{\mu}^{\perp} k_{\nu}^{\perp}-\frac{g_{\mu \nu}^{\perp}}{2} k_{\perp}^{2}\left(q-k_{\perp}\right)^{2}\right] \frac{1}{m^{2}} H_{A}^{f}\left(q, k_{\perp}\right)\right]
\end{aligned}
$$

where we introduced the notation

$$
H_{A}^{f}\left(q, k_{\perp}\right) \equiv h_{A}^{f}\left(\alpha_{q}, k_{\perp}\right) \bar{h}_{A}^{f}\left(\beta_{q},(q-k)_{\perp}\right)+h_{A}^{f} \leftrightarrow \bar{h}_{A}^{f}
$$

Next, consider the case when both $\mu$ and $\nu$ are longitudinal. The non-vanishing terms are

$$
\begin{aligned}
\check{V}_{1 \mu_{\|} \nu_{\|}}^{(2 \mathrm{a}) \mathrm{H}}(x)= & \frac{4 p_{1 \mu} p_{2 \nu}}{s^{2}} \frac{1}{2 s^{3}}\left\langle\bar{\psi} A_{i}(x) \sigma_{\star \alpha} \not p_{2} \gamma^{j} \frac{1}{\alpha} \psi(0)\right\rangle_{A}\left\langle\bar{\psi} B_{j}(0) \sigma_{\bullet}{ }^{\alpha} \not \not_{1} \gamma^{i} \frac{1}{\beta} \psi(x)\right\rangle_{B} \\
& \frac{4 p_{1 \nu} p_{2 \mu}}{s^{2}} \frac{1}{2 s^{3}}\left\langle\bar{\psi} A_{i}(x) \sigma_{\bullet \alpha} \not p_{2} \gamma^{j} \frac{1}{\alpha} \psi(0)\right\rangle_{A}\left\langle\bar{\psi} B_{j}(0) \sigma_{\star}{ }^{\alpha} \not \not_{1} \gamma^{i} \frac{1}{\beta} \psi(x)\right\rangle_{B} \\
& -\frac{4 p_{2 \mu} p_{1 \nu}}{s^{2}} \frac{1}{2 s^{3}}\left\langle\bar{\psi} A^{i}(x) \sigma_{\bullet k} \sigma_{\star j} \frac{1}{\alpha} \psi(0)\right\rangle_{A}\left\langle\bar{\psi} B^{j}(0) \sigma_{\star}{ }_{\star} \sigma_{\bullet} i \frac{1}{\beta} \psi(x)\right\rangle_{B}+\mu \leftrightarrow \nu+x \leftrightarrow 0
\end{aligned}
$$

Using eq. (A.9) it is easy to see that the third term in the r.h.s. is $\sim \frac{q_{\perp}^{2}}{s}$ while the first two terms in the r.h.s. can be rewritten as

$$
\begin{aligned}
& \frac{g_{\mu \nu}^{\|}}{s^{3}}\left\langle\bar{\psi} A^{i}(x) \sigma_{\star j} \frac{1}{\alpha} \psi(0)\right\rangle_{A}\left\langle\bar{\psi} B^{j}(0) \sigma_{\bullet} \frac{1}{\beta} \psi(x)\right\rangle_{B}+x \leftrightarrow 0 \\
& =\frac{g_{\mu \nu}^{\|}}{2 s^{3}}\left\langle\bar{\psi} A^{i}(x) \sigma_{\star i} \frac{1}{\alpha} \psi(0)\right\rangle_{A}\left\langle\bar{\psi} B^{j}(0) \sigma_{\bullet j} \frac{1}{\beta} \psi(x)\right\rangle_{B} \\
& \quad+\frac{g_{\mu \nu}^{\|}}{s^{3}}\left\langle\bar{\psi}\left(A^{i}(x) \sigma_{\star j}-\frac{g_{i j}}{2} A^{k}(x) \sigma_{\star k}\right) \frac{1}{\alpha} \psi(0)\right\rangle_{A}\left\langle\bar{\psi} B^{j}(0) \sigma_{\bullet i} \frac{1}{\beta} \psi(x)\right\rangle_{B}+x \leftrightarrow 0
\end{aligned}
$$

The corresponding contribution to $V_{\mu \nu}^{\mathrm{H}}(q)$ yields

$$
\begin{aligned}
V_{1 \mu_{\|} \nu_{\|}}^{(2 \mathrm{a}) \mathrm{H}}(q)= & -\frac{g_{\mu \nu}^{\|}}{2 Q_{\|}^{2}} \int d^{2} k_{\perp} \frac{k_{\perp}^{2}(q-k)_{\perp}^{2}}{m^{2}} H^{f}\left(q, k_{\perp}\right) \\
& -\frac{g_{\mu \nu}^{\|}}{Q_{\|}^{2}} \int \frac{d^{2} k_{\perp}}{m^{2}}\left[(k, q-k)_{\perp}^{2}-\frac{1}{2} k_{\perp}^{2}(q-k)_{\perp}^{2}\right] H_{A}^{f}\left(q, k_{\perp}\right)
\end{aligned}
$$

where again we used QCD equations of motion (A.56), (A.58) and parametrization (A.63). 
The result for $V_{1 \mu \nu}^{(2 \mathrm{a}) \mathrm{H}}(q)$ is the sum of eqs. (4.50) and (4.54):

$$
\begin{aligned}
V_{1 \mu \nu}^{(2 a) \mathrm{H}}(q)= & \frac{g_{\mu \nu}^{\perp}-g_{\mu \nu}^{\|}}{Q_{\|}^{2}} \int d^{2} k_{\perp} \frac{k_{\perp}^{2}(q-k)_{\perp}^{2}}{2 m^{2}} H^{f}\left(q, k_{\perp}\right) \\
& +\frac{1}{Q_{\|}^{2}} \int d^{2} k_{\perp} \frac{1}{m^{2}}\left\{\left[k_{\mu}^{\perp}(q-k)_{\nu}^{\perp}+\mu \leftrightarrow \nu\right](k, q-k)_{\perp}-k_{\perp}^{2}(q-k)_{\mu}^{\perp}(q-k)_{\nu}^{\perp}\right. \\
& \left.-\left(q-k_{\perp}\right)^{2} k_{\mu}^{\perp} k_{\nu}^{\perp}-\frac{g_{\mu \nu}^{\perp}}{2} k_{\perp}^{2}\left(q-k_{\perp}\right)^{2}-g_{\mu \nu}^{\|}\left[(k, q-k)_{\perp}^{2}-\frac{1}{2} k_{\perp}^{2}(q-k)_{\perp}^{2}\right]\right\} H_{A}^{f}\left(q, k_{\perp}\right)
\end{aligned}
$$

It is possible to demonstrate that the contribution of

$$
\check{V}_{2 \mu \nu}^{(2 \mathrm{a}) \mathrm{H}}(x)=\frac{N_{c}}{2 s}\langle A, B|\left[\psi_{A}^{m}(x) \sigma_{\mu \xi} \Xi_{1}^{n}(0)\right]\left[\psi_{B}^{n}(0) \sigma_{\nu}{ }^{\xi} \Xi_{2}^{m}(x)|A, B\rangle+\mu \leftrightarrow \nu+x \leftrightarrow 0\right.
$$

to $V_{\mu \nu}(q)$ doubles the result (4.55) (see ref. [16] for proof) so subtracting trace we obtain

$$
\begin{aligned}
W_{\mu \nu}^{(2 \mathrm{a}) \mathrm{T}}(q)= & 2 V_{1 \mu \nu}^{(2 \mathrm{a}) \mathrm{H}}(q)-\frac{1}{2} g_{\mu \nu} 2 V_{1 \xi}^{(2) \mathrm{H}, \xi}(q) \\
= & \frac{g_{\mu \nu}^{\perp}-g_{\mu \nu}^{\|}}{Q_{\|}^{2}} \int d^{2} k_{\perp} \frac{1}{m^{2}} k_{\perp}^{2}(q-k)_{\perp}^{2} H^{f}\left(q, k_{\perp}\right) \\
& +\frac{2}{Q_{\|}^{2} N_{c}} \int d^{2} k_{\perp} \frac{1}{m^{2}}\left\{\left[k_{\mu}^{\perp}(q-k)_{\nu}^{\perp}+\mu \leftrightarrow \nu\right](k, q-k)_{\perp}-k_{\perp}^{2}(q-k)_{\mu}^{\perp}(q-k)_{\nu}^{\perp}\right. \\
& \left.\left.-\left(q-k_{\perp}\right)^{2} k_{\mu}^{\perp} k_{\nu}^{\perp}+g_{\mu \nu}^{\perp}(k, q-k)_{\perp}^{2}-g_{\mu \nu}^{\perp} k_{\perp}^{2}\left(q-k_{\perp}\right)^{2}\right]\right\} H_{A}\left(q, k_{\perp}\right)
\end{aligned}
$$

As we will see below, cancellation of terms $\sim g_{\mu \nu}^{\|}$proportional to $H_{A}$ in the r.h.s. of this equation is actually a consequence of (EM) gauge invariance.

Let us now consider

$$
\begin{aligned}
\check{V}_{1 \mu \nu}^{(2 \mathrm{~b}) \mathrm{H}}(x)= & \frac{N_{c}}{2 s}\langle A, B|\left[\psi_{A}^{m}(x) \sigma_{\mu \xi} \psi_{A}^{n}(0)\right]\left[\bar{\Xi}_{2}^{n}(0) \sigma_{\nu}{ }^{\xi} \Xi_{2}^{m}(x)|A, B\rangle+\mu \leftrightarrow \nu+x \leftrightarrow 0\right. \\
= & \frac{1}{s^{3}}\left\{-p_{1 \mu}\left\langle\bar{\psi}(x) A_{j}(x) \sigma_{\nu k} A_{i}(0) \psi(0)\right\rangle_{A}\left\langle\left(\bar{\psi} \frac{1}{\beta}\right)(0) \gamma^{i} \sigma_{\bullet}{ }^{k} \gamma^{j} \frac{1}{\beta} \psi(x)\right\rangle_{B}\right. \\
& \left.+\left\langle\bar{\psi}(x) A_{j}(x) \sigma_{\mu \bullet} A_{i}(0) \psi(0)\right\rangle_{A}\left\langle\left(\bar{\psi} \frac{1}{\beta}\right)(0) \gamma^{i} \sigma_{\bullet \nu_{\perp}} \gamma^{j} \frac{1}{\beta} \psi(x)\right\rangle_{B}\right\}+\mu \leftrightarrow \nu+x \leftrightarrow 0 \\
= & -\frac{4 p_{1 \mu} p_{1 \nu}}{s^{4}}\left\langle\bar{\psi}(x) A_{j}(x) \sigma_{\star k} A_{i}(0) \psi(0)\right\rangle_{A}\left\langle\left(\bar{\psi} \frac{1}{\beta}\right)(0) \gamma^{i} \sigma_{\bullet}{ }^{k} \gamma^{j} \frac{1}{\beta} \psi(x)\right\rangle_{B}
\end{aligned}
$$

where we neglected terms shown in ref. [16] to be small. Using eq. (A.14) the r.h.s. of this equation can be rewritten as

$$
\check{V}_{1 \mu \nu}^{(2 \mathrm{~b}) \mathrm{H}}(x)=-\frac{4 p_{1 \mu} p_{1 \nu}}{s^{4}}\left\langle\bar{\psi}(x) \not A(x) \sigma_{\star k} \not \mathcal{A}(0) \psi(0)\right\rangle_{A}\left\langle\left(\bar{\psi} \frac{1}{\beta}\right)(0) \sigma_{\bullet}{ }^{k} \frac{1}{\beta} \psi(x)\right\rangle_{B}+x \leftrightarrow 0
$$

so the corresponding contribution to $W_{\mu \nu}$ takes the form

$$
V_{1 \mu \nu}^{(2 \mathrm{~b}) \mathrm{H}}(q)=-\frac{4 p_{1 \mu} p_{1 \nu}}{\beta_{q}^{2} s^{2}} \int d^{2} k_{\perp} \frac{1}{m^{2}} k_{\perp}^{2}(k, q-k)_{\perp} H^{f}\left(q, k_{\perp}\right)
$$

where we used eqs. (A.46) and (A.61). 
Next, similarly to eq. (4.45), the contribution

$$
\check{V}_{2 \mu \nu}^{(2 \mathrm{~b}) \mathrm{H}}(x)=\frac{N_{c}}{2 s}\langle A, B|\left[\bar{\Xi}_{1}^{m}(x) \sigma_{\mu \xi} \Xi_{1}^{n}(0)\right]\left[\bar{\psi}_{B}^{n}(0) \sigma_{\nu}{ }^{\xi} \psi_{B}^{m}(x)|A, B\rangle+\mu \leftrightarrow \nu+x \leftrightarrow 0\right.
$$

is obtained by the projectile $\leftrightarrow$ target replacement, namely $p_{1} \leftrightarrow p_{2}, \alpha_{q} \leftrightarrow \beta_{q}$ and $k_{\perp} \leftrightarrow$ $(q-k)_{\perp}$, and we get contribution to $W_{\mu \nu}^{\mathrm{T}}$ in the form

$$
\begin{aligned}
W_{\mu \nu}^{(2 \mathrm{~b}) \mathrm{T}}(q) & =V_{1 \mu \nu}^{(2 \mathrm{~b}) \mathrm{H}}(q)+V_{2 \mu \nu}^{(2 \mathrm{~b}) \mathrm{H}}(q) \\
& =-\int d^{2} k_{\perp}\left[\frac{4 p_{1 \mu} p_{1 \nu}}{\beta_{q}^{2} s^{2}} \frac{k_{\perp}^{2}}{m^{2}}+\frac{4 p_{2 \mu} p_{2 \nu}}{\alpha_{q}^{2} s^{2}} \frac{(q-k)_{\perp}^{2}}{m^{2}}\right](k, q-k)_{\perp} H\left(q, k_{\perp}\right)
\end{aligned}
$$

Finally, it is easy to see that the two-gluon terms

$$
\begin{aligned}
\check{W}_{\mu \nu}^{(2 \mathrm{c}) \mathrm{T}}(x)= & \frac{N_{c}}{2 s}\left(g_{\mu \nu} g^{\alpha \beta}-\delta_{\mu}^{\alpha} \delta_{\nu}^{\beta}-\delta_{\nu}^{\alpha} \delta_{\mu}^{\beta}\right)\langle A, B|\left[\bar{\Xi}_{1}^{m}(x) \sigma_{\alpha \xi} \psi_{A}^{n}(0)\right]\left[\psi_{B}^{n}(0) \sigma_{\beta}^{\xi} \Xi_{2}^{m}(x)\right] \\
& +\left[\psi_{A}^{m}(x) \sigma_{\alpha \xi} \Xi_{1}^{n}(0)\right]\left[\Xi_{2}^{n}(0) \sigma_{\beta}^{\xi} \psi_{B}^{m}(x)\right]|A, B\rangle+x \leftrightarrow 0
\end{aligned}
$$

are $O\left(\frac{1}{N_{c}^{2}}\right)$ so we neglect them.

Summarizing, we get

$$
W_{\mu \nu}^{\mathrm{T}}(q)=\text { eq. }(4.45)+\text { eq. }(4.57)+\text { eq. }(4.62)
$$

and adding $W_{\mu \nu}^{\mathrm{G}}(q)$ from eq. (4.35) we finally get

$$
W_{\mu \nu}^{\mathrm{H}}(q)=W_{\mu \nu}^{\mathrm{H}}(q)+W_{\mu \nu}^{\mathrm{H} 2}(q)
$$

The first, gauge-invariant, part is given by

$$
W_{\mu \nu}^{\mathrm{H}}(q)=\sum_{f} e_{f}^{2} W_{\mu \nu}^{\mathrm{H} f}(q), \quad W_{\mu \nu}^{\mathrm{H} f}(q)=\int d^{2} k_{\perp} H^{f}\left(q, k_{\perp}\right) \mathcal{W}_{\mu \nu}^{H}\left(q, k_{\perp}\right)
$$

where $H^{f}$ is given by eq. (4.36) and

$$
\begin{aligned}
m^{2} \mathcal{W}_{\mu \nu}^{H}\left(q, k_{\perp}\right)= & -k_{\mu}^{\perp}(q-k)_{\nu}^{\perp}-k_{\nu}^{\perp}(q-k)_{\mu}^{\perp}-g_{\mu \nu}^{\perp}(k, q-k)_{\perp}+2 \frac{\tilde{q}_{\mu} \tilde{q}_{\nu}-q_{\mu}^{\|} q_{\nu}^{\|}}{Q_{\|}^{4}} k_{\perp}^{2}(q-k)_{\perp}^{2} \\
& -\left(\frac{q_{\mu}^{\|}}{Q_{\|}^{2}}\left[k_{\perp}^{2}(q-k)_{\nu}^{\perp}+k_{\nu}^{\perp}(q-k)_{\perp}^{2}\right]+\frac{\tilde{q}_{\mu}}{Q_{\|}^{2}}\left[k_{\perp}^{2}(q-k)_{\nu}^{\perp}-k_{\nu}^{\perp}(q-k)_{\perp}^{2}\right]+\mu \leftrightarrow \nu\right) \\
& -\frac{\tilde{q}_{\mu} \tilde{q}_{\nu}+q_{\mu}^{\|} q_{\nu}^{\|}}{Q_{\|}^{4}}\left[q_{\perp}^{2}-2(k, q-k)_{\perp}\right](k, q-k)_{\perp}-\frac{q_{\mu}^{\|} \tilde{q}_{\nu}+\tilde{q}_{\mu} q_{\nu}^{\|}}{Q_{\|}^{4}}(2 k-q, q)_{\perp}(k, q-k)_{\perp}
\end{aligned}
$$

where $q_{\mu}^{\|} \equiv \alpha_{q} p_{1}+\beta_{q} p_{2}$ and $\tilde{q}_{\mu} \equiv \alpha_{q} p_{1}-\beta_{q} p_{2}$. It is easy to see that $q^{\mu} W_{\mu \nu}^{\mathrm{H}}=0$.

The second part is

$$
\begin{aligned}
W_{\mu \nu}^{2 \mathrm{H}}(q)= & \sum_{f} e_{f}^{2} W_{\mu \nu}^{\mathrm{H} 2 f}(q), \\
W_{\mu \nu}^{\mathrm{H} 2 f}(q)= & \frac{1}{Q^{2}} \int d^{2} k_{\perp}\left[\frac { 1 } { m ^ { 2 } } \left\{\left[k_{\mu}^{\perp}(q-k)_{\nu}^{\perp}+\mu \leftrightarrow \nu\right](k, q-k)_{\perp}-k_{\perp}^{2}(q-k)_{\mu}^{\perp}(q-k)_{\nu}^{\perp}\right.\right. \\
& \left.\left.\left.-\left(q-k_{\perp}\right)^{2} k_{\mu}^{\perp} k_{\nu}^{\perp}+g_{\mu \nu}^{\perp}(k, q-k)_{\perp}^{2}-g_{\mu \nu}^{\perp} k_{\perp}^{2}\left(q-k_{\perp}\right)^{2}\right]\right\} H_{A}^{f}\left(q, k_{\perp}\right)+O\left(\frac{1}{N_{c}}\right)\right]+O\left(\frac{Q_{\perp}^{4}}{Q^{4}}\right)
\end{aligned}
$$


where $H_{A}$ is given by eq. (4.51). These terms are not gauge invariant: $q^{\mu} W_{\mu \nu}^{2}(q) \neq 0$. The reason is that gauge invariance is restored after adding terms like $\frac{m_{\perp}^{2}}{Q^{2}} \times$ eq. (3.7) which we do not calculate in this paper. Indeed, for example,

$$
q^{\mu} W_{\mu \nu}^{2}(q) \sim \frac{q_{\nu}^{\perp} q_{\perp}^{2}}{\alpha_{q} \beta_{q} s} \quad \text { and } \quad q^{\mu} \times \frac{p_{2}^{\mu} q_{\perp}^{\nu} q_{\perp}^{2}}{\alpha_{q}^{2} \beta_{q} s^{2}}=\frac{q_{\nu}^{\perp} q_{\perp}^{2}}{\alpha_{q} \beta_{q} s}
$$

They are of the same order so one should expect that gauge invariance is restored after calculation of the terms $\sim \frac{p_{2}^{\mu} q_{\perp}^{\nu} q_{\perp}^{2}}{\alpha_{q}^{2} \beta_{q} s^{2}}$ which are beyond the scope of this paper. For the same reason we see that all structures in eq. (3.7) except $\frac{g_{\mu \nu}^{\perp} q_{\perp}^{2}}{\alpha_{q} \beta_{q} s}$ and $\frac{q_{\mu}^{\perp} q_{\nu}^{\perp}}{\alpha_{q} \beta_{q} s}$ are determined by leading-twist TMDs $f_{1}$ and $h_{1}^{\perp}$.

\subsection{Exchange-type power corrections from $J_{A}^{\mu}(x) J_{B}^{\nu}(0)$ terms}

Power corrections of the "exchange" type come from the terms

$$
\check{W}_{\mu \nu}^{\operatorname{exf} f^{\prime}}(x)=\frac{N_{c}}{s}\left\langle p_{A}, p_{B}\left|\left[\bar{\Psi}_{1}^{f}(x) \gamma_{\mu} \Psi_{1}^{f}(x)\right]\left[\bar{\Psi}_{2}^{f^{\prime}}(0) \gamma_{\nu} \Psi_{2}^{f^{\prime}}(0)\right]+\mu \leftrightarrow \nu\right| p_{A}, p_{B}\right\rangle+x \leftrightarrow 0
$$

where $\Psi_{1}$ and $\Psi_{2}$ are given by eq. (3.4). As demonstrated in ref. [16], the nonzero contributions are

$$
\begin{aligned}
\check{W}_{\mu \nu}^{\operatorname{ex} f f^{\prime}}= & \frac{N_{c}}{s}\left\langle p_{A}, p_{B}\right|\left[\bar{\Xi}_{1}(x) \gamma_{\mu} \psi_{A}(x)\right]^{f}\left[\bar{\psi}_{B}(0) \gamma_{\nu} \Xi_{2}(0)\right]^{f^{\prime}} \\
& +\left[\bar{\psi}_{A}(x) \gamma_{\mu} \Xi_{1}(x)\right]^{f}\left[\bar{\Xi}_{2}(0) \gamma_{\nu} \psi_{B}(0)\right]^{f^{\prime}}+\left[\bar{\Xi}_{1}(x) \gamma_{\mu} \psi_{A}(x)\right]^{f}\left[\bar{\Xi}_{2}(0) \gamma_{\nu} \psi_{B}(0)\right]^{f^{\prime}} \\
& +\left[\bar{\psi}_{A}(x) \gamma_{\mu} \Xi_{1}(x)\right]^{f}\left[\bar{\psi}_{B}(0) \gamma_{\nu} \Xi_{2}(0)\right]^{f^{\prime}}+\mu \leftrightarrow \nu\left|p_{A}, p_{B}\right\rangle+x \leftrightarrow 0
\end{aligned}
$$

with transverse $\mu$ and $\nu$.

It is convenient to calculate traceless part and trace separately. Let us start from traceless part. Separating color-singlet contributions with the help of the formula

$$
\left\langle\bar{\psi}_{m} A_{i}^{a} \psi_{n}\right\rangle=\frac{2 t_{n m}^{a}}{N_{c}^{2}-1}\left\langle\bar{\psi} A_{i} \psi\right\rangle
$$

and using eq. (A.32), we get the corresponding term in $\check{W}_{\mu \nu}$ in the form

$$
\begin{aligned}
\check{W}_{\mu \nu}^{\mathrm{ex} f f^{\prime}}-\frac{g_{\mu \nu}^{\perp}}{2}\left(\check{W}^{\mathrm{ex}, f f^{\prime}}\right)_{m}^{m} \\
=\frac{N_{c}}{\left(N_{c}^{2}-1\right) s^{3}}\left(\left\langle\left(\bar{\psi} \frac{1}{\alpha}\right)(x) \not p_{2} \breve{A}_{\mu}(0) \psi(x)+\bar{\psi}(x) \breve{A}_{\mu}(0) \not p_{2} \frac{1}{\alpha} \psi(x)\right\rangle_{A}^{f}\right. \\
\left.\quad \times\left\langle\left(\bar{\psi} \frac{1}{\beta}\right)(0) \not \not_{1} \breve{B}_{\nu}(x) \psi(0)+\bar{\psi}(0) \breve{B}_{\nu}(x) \not p_{1} \frac{1}{\beta} \psi(0)\right\rangle_{B}^{f^{\prime}}+\mu \leftrightarrow \nu-\text { trace }\right)+x \leftrightarrow 0
\end{aligned}
$$

where we used notations

$$
\breve{A}_{i} \equiv A_{i}-i \tilde{A}_{i} \gamma_{5} . \quad \breve{B}_{i} \equiv B_{i}-i \tilde{B}_{i} \gamma_{5}
$$


Using parametrization of matrix elements (A.66) and (A.67) we get

$$
\begin{aligned}
& W_{\mu \nu}^{\operatorname{ex} f f^{\prime}}(q)-\operatorname{trace}=\frac{s / 2}{(2 \pi)^{4}} \int d^{4} x e^{-i q x}\left(\check{W}_{\mu \nu}^{\operatorname{ex} f f^{\prime}}(x)-\text { trace }\right), \\
& \quad=\frac{N_{c}}{\left(N_{c}^{2}-1\right) Q_{\|}^{2}} \int d^{2} k_{\perp}\left[k_{\mu}^{\perp}(q-k)_{\nu}^{\perp}+\mu \leftrightarrow \nu+g_{\mu \nu}^{\perp}(k, q-k)_{\perp}\right] J_{--}^{1 f f^{\prime}}\left(q, k_{\perp}\right)
\end{aligned}
$$

where $J_{--}^{i f f^{\prime}}\left(q, k_{\perp}\right)$ are defined in eq. (A.71).

The trace part can be obtained in a similar way. Using eq. (A.31) one gets

$$
\begin{aligned}
g^{m n} \check{W}_{m n}^{\text {ex } f f^{\prime}}= & \frac{2 N_{c}}{\left(N_{c}^{2}-1\right) s^{3}}\left(\left\langle\left(\bar{\psi} \frac{1}{\alpha}\right)(x) \breve{A}_{m}(0) \not p_{2} \psi(x)+\bar{\psi}(x) \not p_{2} \breve{A}_{m}(0) \frac{1}{\alpha} \psi(x)\right\rangle_{A}^{f}\right. \\
& \left.\left.\times\left\langle\left(\bar{\psi} \frac{1}{\beta}\right)(0) \breve{B}^{m}(x) \not p_{1} \psi(0)\right\rangle_{B}^{f^{\prime}}+\bar{\psi}(0) \not p_{1} \breve{B}^{m}(x) \frac{1}{\beta} \psi(0)\right\rangle_{B}^{f^{\prime}}\right)+x \leftrightarrow 0
\end{aligned}
$$

The corresponding contribution to trace part of $W\left(\alpha_{q}, \beta_{q}, x_{\perp}\right)$ takes the form

$$
\begin{aligned}
\frac{1}{2} g^{m n} W_{m n}^{\operatorname{ex} f f^{\prime}}(q) & =\frac{s / 4}{(2 \pi)^{4}} \int d^{4} x e^{-i q x} g^{m n} \check{W}_{m n}^{\operatorname{ex} f f^{\prime}}\left(x_{\perp}\right) \\
& =-\frac{N_{c}}{\left(N_{c}^{2}-1\right) Q_{\|}^{2}} \int d^{2} k_{\perp}(k, q-k)_{\perp} J_{--}^{2 f f^{\prime}}\left(q, k_{\perp}\right)
\end{aligned}
$$

which agrees with eq. (6.2) from ref. [14] after replacements $j_{2}=j_{2}^{\text {tw3 }}-i \tilde{j}_{2}^{\text {tw3 }}$ and $\bar{j}_{2}=$ $j_{1}^{\mathrm{tw} 3}+i \tilde{j}_{1}^{\mathrm{tw} 3}$. It should be noted that the difference between $j_{1}$ and $j_{2}$ in traceless $v s$ trace part is due to difference in formulas (A.32) and (A.31).

The total contribution of "exchange" power corrections is the sum of eqs. (4.75) and (4.77), see eq. (4.80) below.

\subsection{Resulting hadronic tensor for photon-mediated DY process}

It is convenient to represent hadronic tensor as a sum of three parts

$$
W_{\mu \nu}^{\gamma}(q)=W_{\mu \nu}^{1}(q)+W_{\mu \nu}^{2 \mathrm{H}}(q)+W_{\mu \nu}^{3}(q)
$$

The first, gauge-invariant, part has the form

$$
\begin{aligned}
W_{\mu \nu}^{1}(q) & =\sum_{f} e_{f}^{2}\left[W_{\mu \nu}^{\mathrm{F} f}(q)+W_{\mu \nu}^{\mathrm{H} f}(q)\right] \\
W_{\mu \nu}^{\mathrm{F} f}(q) & =\int d^{2} k_{\perp} F^{f}\left(q, k_{\perp}\right) \mathcal{W}_{\mu \nu}^{F}\left(q, k_{\perp}\right), \\
W_{\mu \nu}^{\mathrm{H} f}(q) & =\int d^{2} k_{\perp} H^{f}\left(q, k_{\perp}\right) \mathcal{W}_{\mu \nu}^{H}\left(q, k_{\perp}\right)
\end{aligned}
$$

where functions $F^{f}\left(q, k_{\perp}\right)$ and $H^{f}\left(q, k_{\perp}\right)$ are given by eqs. (4.12) and (4.36) while

$\mathcal{W}_{\mu \nu}^{\mathrm{F}}\left(q, k_{\perp}\right)$ and $\mathcal{W}_{\mu \nu}^{\mathrm{H}}\left(q, k_{\perp}\right)$ are presented in eqs. (4.31) and (4.67), respectively. ${ }^{7}$ Note that $q^{\mu} W_{\mu \nu}^{\mathrm{F}}$ and $q^{\mu} W_{\mu \nu}^{\mathrm{H}}$ are exactly zero without any $\frac{q_{\perp}^{2}}{Q^{2}}$ corrections. This is similar to usual

\footnotetext{
${ }^{7}$ It should be mentioned that $W^{F}$ part coincides with the result obtained in refs. [22, 23] using parton Reggeization approach to DY process [24].
} 
"forward" DIS, but different from off-forward DVCS where the cancellations of right-hand sides of Ward identities involve infinite towers of twists [25-27]

The second part is given by eq. (4.68). It has the same order in $N_{c}$ as the first part but unfortunately is determined by quark-quark-gluon TMDs which are virtually unknown.

The third, "exchange", part is given by the sum of eqs. (4.75) and (4.77).

$$
\begin{aligned}
W_{\mu \nu}^{\mathrm{ex}}(q) & =\sum_{f, f^{\prime}} e_{f} e_{f^{\prime}} W_{\mu \nu}^{\operatorname{ex} f f^{\prime}}(q) \\
W_{\mu \nu}^{\operatorname{ex} f f^{\prime}}(q) & =\frac{N_{c}}{\left(N_{c}^{2}-1\right) Q_{\|}^{2}} \int d^{2} k_{\perp}\left\{\left[k_{\mu}^{\perp}(q-k)_{\nu}^{\perp}+\mu \leftrightarrow \nu+g_{\mu \nu}^{\perp}(k, q-k)_{\perp}\right] J_{--}^{1 f f^{\prime}}\left(q, k_{\perp}\right)\right. \\
& \left.-g_{\mu \nu}^{\perp}(k, q-k)_{\perp} J_{--}^{2 f f^{\prime}}\left(q, k_{\perp}\right)\right\}
\end{aligned}
$$

Both second and third part come only from transverse indices.

These terms are not gauge invariant: $q^{\mu} W_{\mu \nu}^{2}(q) \neq 0$. The reason is that gauge invariance is restored after adding terms like $\frac{m_{\perp}^{2}}{Q^{2}} \times$ eq. (3.7) which we do not calculate in this paper. Indeed, for example,

$$
q^{\mu} W_{\mu \nu}^{2,3}(q) \sim \frac{q_{\nu}^{\perp} q_{\perp}^{2}}{\alpha_{q} \beta_{q} s} \quad \text { and } \quad q^{\mu} \times \frac{p_{2}^{\mu} q_{\perp}^{\nu} q_{\perp}^{2}}{\alpha_{q}^{2} \beta_{q} s^{2}}=\frac{q_{\nu}^{\perp} q_{\perp}^{2}}{\alpha_{q} \beta_{q} s}
$$

They are of the same order so one should expect that gauge invariance is restored after calculation of the terms $\sim \frac{p_{2}^{\mu} q_{\perp}^{\nu} q_{\perp}^{2}}{\alpha_{q}^{2} \beta_{q} s^{2}}$ which are beyond the scope of this paper. For the same reason we see that all structures in eq. (3.7) except $\frac{g_{\mu \nu}^{\perp} q_{\perp}^{2}}{\alpha_{q} \beta_{q} s}$ and $\frac{q_{\mu}^{\perp} q_{\nu}^{\perp}}{\alpha_{q} \beta_{q} s}$ are determined by leading-twist TMDs $f_{1}$ and $h_{1}^{\perp}$.

In the remaining sections we will use formulas from this section as guidelines for calculation of other hadronic tensors in eq. (2.6).

\section{$5 \quad Z$-mediated hadronic tensor}

In this section we will consider the hadronic tensor corresponding to the part of DY cross section mediated by $Z$-boson. Let us start from the symmetric tensor $W_{\mu \nu}^{\mathrm{ZS}}$ defined in eq. (2.9)

\subsection{Symmetric part of $Z$-mediated hadronic tensor}

As in the photon case, after integration over central fields $\mathcal{J}^{\mu}$ in eq. (2.4) is replaced by

$$
\begin{aligned}
-\mathcal{J}^{\mu} & =\mathcal{J}_{1}^{\mu}+\mathcal{J}_{2}^{\mu}+\mathcal{J}_{12}^{\mu}+\mathcal{J}_{21}^{\mu}, \\
\mathcal{J}_{1}^{\mu} & =\sum_{f} c_{f} \bar{\Psi}_{1}^{f} \gamma_{\mu}\left(a_{f}-\gamma_{5}\right) \Psi_{1}^{f}, \quad \mathcal{J}_{12}^{\mu}=\sum_{f} c_{f} \bar{\Psi}_{1}^{f} \gamma_{\mu}\left(a_{f}-\gamma_{5}\right) \Psi_{2}^{f}
\end{aligned}
$$

and similarly for $\mathcal{J}_{2}^{\mu}$ and $\mathcal{J}_{21}^{\mu}$.

We start from "annihilation-type" contributions and consider

$$
\check{W}_{\mu \nu}^{\mathrm{ZSa}}=\frac{N_{c}}{s}\left\langle A, B\left|\mathcal{J}_{12}^{\mu}(x) \mathcal{J}_{21}^{\mu}(0)+\mu \leftrightarrow \nu\right| A, B\right\rangle+x \leftrightarrow 0
$$


Similarly to the photon case, we will perform calculations for one flavor and sum over flavors later. After Fierz transformations (A.1), (A.3) one gets the hadronic tensor (5.2) in the form

$$
\begin{aligned}
c_{f}^{-2} \check{W}_{\mu \nu}^{\mathrm{ZSa}=} & \frac{N_{c}}{s}\langle A, B| a_{f}^{2} \Psi_{1} \gamma_{\mu} \Psi_{2}(x) \Psi_{2} \gamma_{\nu} \Psi_{1}(0)+\Psi_{1} \gamma_{\mu} \gamma_{5} \Psi_{2}(x) \Psi_{2} \gamma_{\nu} \gamma_{5} \Psi_{1}(0) \\
& -a_{f} \Psi_{1} \gamma_{\mu} \Psi_{2}(x) \Psi_{2} \gamma_{\nu} \gamma_{5} \Psi_{1}(0)-a_{f} \Psi_{1} \gamma_{\mu} \gamma_{5} \Psi_{2}(x) \Psi_{2} \gamma_{\nu} \Psi_{1}(0)|A, B\rangle+\mu \leftrightarrow \nu+x \leftrightarrow 0 \\
= & \left(a_{f}^{2}+1\right) \check{W}_{\mu \nu}^{\mathrm{F} f}(x)+\left(a_{f}^{2}-1\right) \check{W}_{\mu \nu}^{\mathrm{H} f}(x)-2 a_{f} \check{W}_{\mu \nu}^{5 f}(x)
\end{aligned}
$$

where $W_{\mu \nu}^{\mathrm{F} f}(x)$ and $W_{\mu \nu}^{\mathrm{H} f}(x)$ are defined by eqs. (4.6) and (4.7) whereas

$$
\begin{aligned}
\check{W}_{\mu \nu}^{5}(x)= & \frac{N_{c}}{2 s}\left(g_{\mu \nu} g^{\alpha \beta}-\delta_{\mu}^{\alpha} \delta_{\nu}^{\beta}-\delta_{\nu}^{\alpha} \delta_{\mu}^{\beta}\right)\left\{\left[\bar{\Psi}_{1}^{m}(x) \gamma_{\alpha} \gamma_{5} \Psi_{1}^{n}(0)\right]\left[\bar{\Psi}_{2}^{n}(0) \gamma^{\beta} \Psi_{2}^{m}(x)\right]\right. \\
& \left.+\gamma_{\alpha} \gamma_{5} \otimes \gamma_{\beta} \leftrightarrow \gamma_{\alpha} \otimes \gamma_{\beta} \gamma_{5}\right\}+x \leftrightarrow 0 .
\end{aligned}
$$

for flavor $f$ which we are considering. The results for $W_{\mu \nu}^{\mathrm{F} f}(q)$ and $W_{\mu \nu}^{\mathrm{H} f}(q)$ are given by eqs. (4.30) and (4.65) from previous section while $W_{\mu \nu}^{5 f}(q)$ must be evaluated anew.

We will prove now that

$$
\check{W}_{\mu \nu}^{5}(x)=0
$$

with our accuracy. It includes the same terms as we assembled to $\check{W}_{\mu \nu}^{\mathrm{F}}(x)$ but with additional $\gamma_{5}$ attached to one of the fermion fields. Since extra $\gamma_{5}$ cannot change the power of $s$ we need to look how the terms which gave leading contribution to $\check{W}_{\mu \nu}^{\mathrm{F}}(x)$ are affected by extra $\gamma_{5}$. First, note that the leading-twist first term in the r.h.s. of eq. (4.10) with extra $\gamma_{5}$ can be neglected. Indeed, if one replaces $\gamma_{\alpha}$ by $\gamma_{\alpha} \gamma_{5}$ (or $\gamma_{\beta}$ by $\gamma_{\beta} \gamma_{5}$ ) the term $\sim g_{\mu \nu}$ vanishes and two other terms are $\sim \frac{1}{s} p_{2 \beta} \epsilon_{\alpha j} q^{j}$ as seen from the parametrization (A.43). Similarly, replacement $\gamma_{\beta} \rightarrow \gamma_{\beta} \gamma_{5}$ gives terms of order of $\sim \frac{1}{s} p_{1 \alpha} \epsilon_{\beta j} q^{j}$ which we neglect, see the discussion after eq. (3.7).

Next, let us consider sum of terms in $\check{W}_{1 \mu \nu}^{(1) \mathrm{F}}(x)$ and $\check{W}_{2 \mu \nu}^{(1) \mathrm{F}}(x)$ which has the form

$$
\begin{gathered}
\frac{p_{2 \mu}}{s^{2}}\left[\left\langle\bar{\psi}(x) \not p_{2} \frac{1}{\alpha} \psi(0)\right\rangle_{A}\left\langle\bar{\psi} \not B(0) \not p_{1} \gamma_{\nu}^{\perp} \psi(x)\right\rangle_{B}+\left\langle\bar{\psi} \frac{1}{\alpha}(x) \not p_{2} \psi(0)\right\rangle_{A}\left\langle\bar{\psi}(0) \gamma_{\nu}^{\perp} \not p_{1} \bar{B}(x) \psi(x)\right\rangle_{B}\right. \\
\left.+\psi(0) \otimes \psi(x) \leftrightarrow \psi(0) \gamma_{5} \otimes \gamma_{5} \psi(x)\right]+\mu \leftrightarrow \nu+x \leftrightarrow 0,
\end{gathered}
$$

see eqs. (4.14) and (4.16). ${ }^{8}$ It is easy to see that the replacement $\psi(0) \rightarrow \gamma_{5} \psi(0)$ gives either vanishing projectile matrix element or vanishing target matrix element after using eqs. (A.45), (A.46), (A.52), and (A.53). Similarly, the sum of contributions to $\check{W}_{3 \mu \nu}^{(1) \mathrm{F}}(x)+$ $\check{W}_{4 \mu \nu}^{(1) \mathrm{F}}(x)$

$$
\begin{aligned}
& \frac{p_{1 \mu}}{s^{2}}\left[\left\langle\bar{\psi} \not A(x) \not p_{2} \gamma_{\nu_{\perp}} \psi(0)\right\rangle_{A}\left\langle\bar{\psi}(0) \not p_{1} \frac{1}{\beta} \psi(x)\right\rangle_{B}+\left\langle\bar{\psi}(x) \gamma_{\nu_{\perp}} \not \not_{2} \not A(0) \psi(0)\right\rangle_{A}\left\langle\left(\bar{\psi} \frac{1}{\beta}\right)(0) \not b_{1} \psi(x)\right\rangle_{B}\right. \\
& \left.\quad+\psi(0) \otimes \psi(x) \leftrightarrow \psi(0) \gamma_{5} \otimes \gamma_{5} \psi(x)\right]+\mu \leftrightarrow \nu+x \leftrightarrow 0
\end{aligned}
$$

\footnotetext{
${ }^{8}$ In these equations we dropped vanishing terms $+\psi(0) \otimes \psi(x) \leftrightarrow \psi(0) \gamma_{5} \otimes \gamma_{5} \psi(x)$ but now we need them.
} 
vanishes after replacement $\psi(0) \rightarrow \gamma_{5} \psi(0)$ due to $\mathrm{QCD}$ equations of motion mentioned above. Thus, $\check{W}_{\mu \nu}^{(1) 5}(x)=0$

Let us now consider leading $W_{\mu \nu}^{(2) \mathrm{F}}$ terms with two gluon operators discussed in section 4.1.2, see eq. (4.18). As we saw, the leading contribution comes from transverse $\mu$ and $\nu$. It is given by sum of eqs. (4.20), (4.21), and the corresponding terms coming from

$$
\begin{aligned}
{\left[\bar{\Xi}_{1}^{m}(x) \gamma_{\alpha} \gamma_{5} \psi_{A}^{n}(0)\right]\left[\bar{\Xi}_{2}^{n}(0) \gamma_{\beta} \gamma_{5} \psi_{B}^{m}(x)\right] } & \\
\check{W}_{1 \mu \nu}^{(2) \mathrm{F}}(x)= & \frac{g_{\mu \nu}^{\|}}{s^{3}}\left(\left\langle\bar{\psi}(x) \not A(x) \not p_{2} \gamma_{i} \frac{1}{\alpha} \psi(0)\right\rangle_{A}\left\langle\bar{\psi}(0) \not B(0) \not p_{1} \gamma^{i} \frac{1}{\beta} \psi(x)\right\rangle_{B}\right. \\
& \left.+\left\langle\left(\bar{\psi} \frac{1}{\alpha}\right)(x) \gamma_{i} \not \not_{2} \not A(0) \psi(0)\right\rangle_{A}\left\langle\left(\bar{\psi} \frac{1}{\beta}\right)(0) \gamma^{i} \not p_{1} \not B(x) \psi(x)\right\rangle_{B}\right)+x \leftrightarrow 0
\end{aligned}
$$

As was mentioned in the end of section 4.1.2, from equations (A.31) it is clear that the second term in the r.h.s. gives the same contribution as the first term. If, however, one replaces $\psi(0) \leftrightarrow \gamma_{5} \psi(0)$ (or, equivalently, $\psi(x) \leftrightarrow \gamma_{5} \psi(x)$ ), it is easy to see that the two contributions cancel so $\check{W}_{\mu \nu}^{(2) \mathrm{F} 5}(x)=0$.

Next, the leading terms in $\check{W}_{2 \mu \nu}^{(2) \mathrm{F}}(x)$ are given by eqs. (4.25) and (4.27)

$$
\begin{aligned}
\check{W}_{2 \mu \nu}^{(2) \mathrm{F}}(x)= & -\frac{4 p_{1 \mu} p_{1 \nu}}{s^{4}}\left(\left\langle\bar{\psi}(x) \not A(x) \not p_{2} \not A(0) \psi(0)\right\rangle_{A}\left\langle\left(\bar{\psi} \frac{1}{\beta}\right)(0) \not p_{1} \frac{1}{\beta} \psi(x)\right\rangle_{B}\right. \\
& \left.+\psi(0) \otimes \psi(x) \leftrightarrow \gamma_{5} \psi(0) \otimes \gamma_{5} \psi(x)\right)-\frac{4 p_{2 \mu} p_{2 \nu}}{s^{4}}\left(\left\langle\bar{\psi}(x) \not B(x) \not p_{1} \not B(0) \psi(0)\right\rangle_{B}\right. \\
& \left.\times\left\langle\left(\bar{\psi} \frac{1}{\alpha}\right)(0) \not p_{2} \frac{1}{\alpha} \psi(x)\right\rangle_{A}+\psi(0) \otimes \psi(x) \leftrightarrow \gamma_{5} \psi(0) \otimes \gamma_{5} \psi(x)\right)+x \leftrightarrow 0
\end{aligned}
$$

If we now replace $\psi(0) \leftrightarrow \gamma_{5} \psi(0)$ it is easy to see from eqs. (A.46) and (A.61) that $\check{W}_{\mu \nu}^{(2 b) 5}(x)=0$. As to $\check{W}_{3 \mu \nu}^{(2) \mathrm{F}}(x)$, from eq. (4.29) we see that it is $O\left(\frac{1}{N_{c}^{2}}\right)$ so we neglect it.

Thus, we obtain the "annihilation part" of symmetric hadronic tensor due to Z-boson currents in the form

$$
W_{\mu \nu}^{\mathrm{ZSan}}=e^{2} \sum_{f} c_{f}^{2}\left[\left(a_{f}^{2}+1\right) W_{\mu \nu}^{f \mathrm{~F}}(q)+\left(a_{f}^{2}-1\right) W_{\mu \nu}^{f \mathrm{H}}(q)\right]
$$

where $W_{\mu \nu}^{f \mathrm{~F}}(q)$ and $W_{\mu \nu}^{f \mathrm{H}}(q)$ are given by eqs. (4.30) and (4.65). Note that it is gauge invariant up to $W_{\mu \nu}^{2 \mathrm{H}}(q)$ term discussed in the end of section 4.

\subsubsection{Exchange-type power corrections to $W_{\mu \nu}^{Z S}$}

Power corrections of the "exchange" type come from the terms

$$
\left(\check{W}_{f f^{\prime}}^{\mathrm{ZS}}\right)_{\mu \nu}^{\mathrm{ex}}(x)=c_{f} c_{f^{\prime}}\left[a_{f} a_{f^{\prime}} \check{W}_{\mu \nu}^{f f^{\prime} \mathrm{ex}}(x)+\left(\check{W}_{55}^{\mathrm{S} f f^{\prime}}\right)_{\mu \nu}^{\mathrm{ex}}(x)-a_{f}\left(\check{W}_{5 \mathrm{a}}^{\mathrm{S} f f^{\prime}}\right)_{\mu \nu}^{\mathrm{ex}}(x)-a_{f^{\prime}}\left(\check{W}_{5 \mathrm{~b}}^{\mathrm{S} f f^{\prime}}\right)_{\mu \nu}^{\mathrm{ex}}(x)\right]
$$


where $\check{\mathcal{W}}_{\mu \nu}^{\text {ex }}(x)$ is given by eq. $(4.71)$ while $\left(\check{\mathcal{W}}_{55}^{\mathrm{S}}\right)_{\mu \nu}^{\mathrm{ex}}(x)\left(\check{\mathcal{W}}_{5 A, B}^{\mathrm{S}}\right)_{\mu \nu}^{\mathrm{ex}}(x)$ are defined as

$$
\begin{aligned}
& \left(\check{W}_{55}^{\mathrm{S} f f^{\prime}}\right)_{\mu \nu}^{\mathrm{ex}}(x) \equiv \frac{N_{c}}{s}\left\langle A, B\left|\left[\bar{\Psi}_{1}(x) \gamma_{\mu} \gamma_{5} \Psi_{1}(x)\right]\left[\bar{\Psi}_{2}(0) \gamma_{\nu} \gamma_{5} \Psi_{2}(0)\right]+\mu \leftrightarrow \nu\right| A, B\right\rangle+x \leftrightarrow 0, \\
& \left(\check{W}_{5 \mathrm{a}}^{\mathrm{S} f f^{\prime}}\right)_{\mu \nu}^{\mathrm{ex}}(x) \equiv \frac{N_{c}}{s}\left\langle A, B\left|\left[\bar{\Psi}_{1}(x) \gamma_{\mu} \gamma_{5} \Psi_{1}(x)\right]\left[\bar{\Psi}_{2}(0) \gamma_{\nu} \Psi_{2}(0)\right]+\mu \leftrightarrow \nu\right| A, B\right\rangle+x \leftrightarrow 0, \\
& \left(\check{W}_{5 \mathrm{~b}}^{\mathrm{S} f f^{\prime}}\right)_{\mu \nu}^{\mathrm{ex}}(x) \equiv \frac{N_{c}}{s}\left\langle A, B\left|\left[\bar{\Psi}_{1}(x) \gamma_{\mu} \Psi_{1}(x)\right]\left[\bar{\Psi}_{2}(0) \gamma_{\nu} \gamma_{5} \Psi_{2}(0)\right]+\mu \leftrightarrow \nu\right| A, B\right\rangle+x \leftrightarrow 0
\end{aligned}
$$

As seen from the of comparison parametrizations (A.66) and (A.70), the replacement $\psi \rightarrow \gamma_{5} \psi$ in the projectile matrix elements leads to $k_{\mu} j_{1} \rightarrow \pm i \epsilon_{\mu \nu} k^{\nu} j_{1}, k_{\mu} j_{2} \rightarrow \pm i \epsilon_{\mu \nu} k^{\nu} j_{2}$. Similarly, the replacement $\psi \rightarrow \gamma_{5} \psi$ in target matrix elements yields $(q-k)_{\mu} j_{1,2} \rightarrow \pm i \epsilon_{\mu}(q-$ $k)^{\nu} j_{1,2}$. Looking at the result (4.80) and taking care of signs of replacements $\psi \rightarrow \gamma_{5} \psi$ in eqs. (A.66) and (A.67), we obtain

$$
\begin{aligned}
& \left(\check{W}_{55}^{\mathrm{S} f f^{\prime}}\right)_{\mu \nu}^{\mathrm{ex}}(x)=\frac{N_{c}}{\left(N_{c}^{2}-1\right) s^{3}}\left(\left\langle\left(\bar{\psi} \frac{1}{\alpha}\right)(x) \not p_{2} \breve{A}_{\mu}(0) \gamma_{5} \psi(x)+\bar{\psi}(x) \breve{A}_{\mu}(0) \not p_{2} \frac{1}{\alpha} \gamma_{5} \psi(x)\right\rangle_{A}^{f}\right. \\
& \left.\times\left\langle\left(\bar{\psi} \frac{1}{\beta}\right)(0) \not p_{1} \breve{B}_{\nu}(x) \gamma_{5} \psi(0)\right\rangle_{B}+\bar{\psi}(0) \breve{B}_{\nu}(x) \not p_{1} \frac{1}{\beta} \gamma_{5} \psi(0)\right\rangle_{B}^{f^{\prime}}+\mu \leftrightarrow \nu \text {-trace } \\
& +g_{\mu \nu}^{\perp}\left\langle\left(\bar{\psi} \frac{1}{\alpha}\right)(x) \breve{A}_{m}(0) \not p_{2} \gamma_{5} \psi(x)+\bar{\psi}(x) \not p_{2} \breve{A}_{m}(0) \frac{1}{\alpha} \gamma_{5} \psi(x)\right\rangle_{A}^{f} \\
& \left.\left.\times\left\langle\left(\bar{\psi} \frac{1}{\beta}\right)(0) \breve{B}^{m}(x) \not p_{1} \gamma_{5} \psi(0)\right\rangle_{B}+\bar{\psi}(0) \not \not_{1} \breve{B}^{m}(x) \frac{1}{\beta} \gamma_{5} \psi(0)\right\rangle_{B}^{f^{\prime}}\right)+x \leftrightarrow 0 \\
& \Rightarrow\left(W_{\mu \nu}^{\mathrm{S} f f^{\prime}}\right)_{55}^{\mathrm{ex}}(q)=-\frac{N_{c}}{\left(N_{c}^{2}-1\right) Q_{\|}^{2}} \int d^{2} k_{\perp}\left\{\left[k_{\mu}^{\perp}(q-k)_{\nu}^{\perp}+\mu \leftrightarrow \nu+g_{\mu \nu}^{\perp}(k, q-k)_{\perp}\right]\right. \\
& \left.\times J_{++}^{1 f f^{\prime}}\left(q, k_{\perp}\right)-g_{\mu \nu}^{\perp}(k, q-k)_{\perp} J_{++}^{2 f f^{\prime}}\left(q, k_{\perp}\right)\right\}
\end{aligned}
$$

and

$$
\begin{aligned}
\left(\breve{\mathcal{W}}_{5 \mathrm{a}}^{\mathrm{S} f f^{\prime}}\right)_{\mu \nu}^{\mathrm{ex}}(x)= & \frac{N_{c}}{\left(N_{c}^{2}-1\right) s^{3}}\left(\left\langle\left(\bar{\psi} \frac{1}{\alpha}\right)(x) \not p_{2} \breve{A}_{\mu}(0) \gamma_{5} \psi(x)+\bar{\psi}(x) \breve{A}_{\mu}(0) \not p_{2} \frac{1}{\alpha} \gamma_{5} \psi(x)\right\rangle_{A}^{f}\right. \\
& \left.\times\left\langle\left(\bar{\psi} \frac{1}{\beta}\right)(0) \not p_{1} \breve{B}_{\nu}(x) \psi(0)\right\rangle_{B}+\bar{\psi}(0) \breve{B}_{\nu}(x) \not p_{1} \frac{1}{\beta} \psi(0)\right\rangle_{B}^{f^{\prime}}+\mu \leftrightarrow \nu-\text { trace } \\
& +g_{\mu \nu}^{\perp}\left\langle\left(\bar{\psi} \frac{1}{\alpha}\right)(x) \breve{A}_{m}(0) \not p_{2} \gamma_{5} \psi(x)+\bar{\psi}(x) \not p_{2} \breve{A}_{m}(0) \frac{1}{\alpha} \gamma_{5} \psi(x)\right\rangle_{A}^{f} \\
& \left.\left.\times\left\langle\left(\bar{\psi} \frac{1}{\beta}\right)(0) \breve{B}^{m}(x) \not p_{1} \gamma_{5} \psi(0)\right\rangle_{B}+\bar{\psi}(0) \not p_{1} \breve{B}^{m}(x) \frac{1}{\beta} \gamma_{5} \psi(0)\right\rangle_{B}^{f^{\prime}}\right)+x \leftrightarrow 0 \\
\Rightarrow & \left(W_{\mu \nu}^{\mathrm{S} f f^{\prime}}\right)_{5 \mathrm{a}}^{\mathrm{ex}}(q)=-\frac{N_{c}}{\left(N_{c}^{2}-1\right) Q_{\|}^{2}} \int d^{2} k_{\perp}\left[\epsilon_{\mu m} k^{m}(q-k)_{\nu}+\mu \leftrightarrow \nu\right] I_{+-}^{1 f f^{\prime}}\left(q, k_{\perp}\right)
\end{aligned}
$$




$$
\begin{aligned}
\left(\check{\mathcal{W}}_{5 \mathrm{~b}}^{\mathrm{S} f f^{\prime}}\right)_{\mu \nu}^{\mathrm{ex}}(x)= & \frac{N_{c}}{\left(N_{c}^{2}-1\right) s^{3}}\left(\left\langle\left(\bar{\psi} \frac{1}{\alpha}\right)(x) \not p_{2} \breve{A}_{\mu}(0) \psi(x)+\bar{\psi}(x) \breve{A}_{\mu}(0) \not p_{2} \frac{1}{\alpha} \psi(x)\right\rangle_{A}^{f}\right. \\
& \left.\times\left\langle\left(\bar{\psi} \frac{1}{\beta}\right)(0) \not p_{1} \breve{B}_{\nu}(x) \gamma_{5} \psi(0)\right\rangle_{B}+\bar{\psi}(0) \breve{B}_{\nu}(x) \not p_{1} \gamma_{5} \frac{1}{\beta} \psi(0)\right\rangle_{B}^{f^{\prime}}+\mu \leftrightarrow \nu-\text { trace } \\
& +g_{\mu \nu}^{\perp}\left\langle\left(\bar{\psi} \frac{1}{\alpha}\right)(x) \breve{A}_{m}(0) \not p_{2} \gamma_{5} \psi(x)+\bar{\psi}(x) \not p_{2} \breve{A}_{m}(0) \frac{1}{\alpha} \gamma_{5} \psi(x)\right\rangle_{A}^{f} \\
& \left.\left.\times\left\langle\left(\bar{\psi} \frac{1}{\beta}\right)(0) \breve{B}^{m}(x) \not p_{1} \gamma_{5} \psi(0)\right\rangle_{B}+\bar{\psi}(0) \not p_{1} \breve{B}^{m}(x) \frac{1}{\beta} \gamma_{5} \psi(0)\right\rangle_{B}^{f^{\prime}}\right)+x \leftrightarrow 0 \\
\Rightarrow & \left(W_{\mu \nu}^{\mathrm{S} f f^{\prime}}\right)_{5 \mathrm{~b}}^{\mathrm{ex}}(q)=\frac{N_{c}}{\left(N_{c}^{2}-1\right) Q_{\|}^{2}} \int d^{2} k_{\perp}\left[k_{\mu} \epsilon_{\nu n}(q-k)^{n}+\mu \leftrightarrow \nu\right] I_{-+}^{1 f f^{\prime}}\left(q, k_{\perp}\right)
\end{aligned}
$$

where

Note that the two last contributions are traceless since

$$
\epsilon_{i j} \int d^{2} k_{\perp} k^{i}(q-k)^{j} \phi_{1}\left(k_{\perp}^{2}\right) \phi_{2}\left((q-k)_{\perp}^{2}\right)=0
$$

for any functions $\phi_{1}$ and $\phi_{2}$.

\subsubsection{Results for symmetric hadronic tensor for Z-mediated DY process}

It is convenient to represent the hadronic tensor $W_{\mu \nu}^{\mathrm{ZS}}$ as a sum of three parts

$$
W_{\mu \nu}^{\mathrm{ZS}}(q)=W_{\mu \nu}^{\mathrm{ZS} 1}(q)+W_{\mu \nu}^{\mathrm{ZS} 2}(q)+W_{\mu \nu}^{\mathrm{ZS} 3}(q)
$$

The first, gauge-invariant, part is given by eq. (5.3)

$$
\begin{aligned}
W_{\mu \nu}^{\mathrm{ZS} 1}(q) & =e^{2} \sum_{f} c_{f}^{2}\left[\left(a_{f}^{2}+1\right) W_{\mu \nu}^{\mathrm{F} f}(q)+\left(a_{f}^{2}-1\right) W_{\mu \nu}^{\mathrm{H} f}(q)\right] \\
W_{\mu \nu}^{\mathrm{fF}}(q) & =\frac{1}{N_{c}} \int d^{2} k_{\perp} F^{f}\left(q, k_{\perp}\right) \mathcal{W}_{\mu \nu}^{F}\left(q, k_{\perp}\right) \\
& =W_{\mu \nu}^{\mathrm{fH}}(q)=\frac{1}{N_{c}} \int d^{2} k_{\perp} H^{f}\left(q, k_{\perp}\right) \mathcal{W}_{\mu \nu}^{H}\left(q, k_{\perp}\right)
\end{aligned}
$$

where $W_{\mu \nu}^{\mathrm{F} f}(q)$ is given by eq. (4.30) and $W_{\mu \nu}^{\mathrm{H} f}(q)$ by eq.

$$
W_{\mu \nu}^{\mathrm{ZSan}}=e^{2} \sum_{f} c_{f}^{2}\left[\left(a_{f}^{2}+1\right) W_{\mu \nu}^{f \mathrm{~F}}(q)+\left(a_{f}^{2}-1\right) W_{\mu \nu}^{f \mathrm{H}}(q)\right]
$$

where $W_{\mu \nu}^{\mathrm{F} f}(q)$ and $W_{\mu \nu}^{\mathrm{Hf}}(q)$ are given by eqs. (4.30) and (4.66).

The second part is

$$
W_{\mu \nu}^{\mathrm{ZS} 2}(q)=\sum_{f} c_{f}^{2}\left(a_{f}^{2}-1\right) W_{\mu \nu}^{2 \mathrm{Hf}}(q)
$$

where $W_{\mu \nu}^{2 \mathrm{Hf}}(q)$ is given by eq. (4.68). 
The third, "exchange", part is given by the sum

$$
\begin{aligned}
& W_{\mu \nu}^{\mathrm{ZSex}}(q)= \\
& \quad \frac{N_{c}}{\left(N_{c}^{2}-1\right) Q_{\|}^{2}} \sum_{f, f^{\prime}} c_{f} c_{f^{\prime}} \int d^{2} k_{\perp}\left\{\left[k_{\mu}^{\perp}(q-k)_{\nu}^{\perp}+\mu \leftrightarrow \nu+g_{\mu \nu}^{\perp}(k, q-k)_{\perp}\right]\right. \\
& \quad \times\left(a_{f} a_{f^{\prime}} J_{--}^{1 f f^{\prime}}-J_{++}^{1 f f^{\prime}}\right)-g_{\mu \nu}^{\perp}(k, q-k)_{\perp}\left(a_{f} a_{f^{\prime}} J_{++}^{2 f f^{\prime}}-J_{++}^{2 f f^{\prime}}\right) \\
& \left.\quad+a_{f}\left[\epsilon_{\mu m} k^{m}(q-k)_{\nu}+\mu \leftrightarrow \nu\right] I_{+-}^{1 f f^{\prime}}\left(q, k_{\perp}\right)-a_{f^{\prime}}\left[k_{\mu} \epsilon_{\nu n}(q-k)^{n}+\mu \leftrightarrow \nu\right] I_{-+}^{1 f f^{\prime}}\left(q, k_{\perp}\right)\right\}
\end{aligned}
$$

where $J_{ \pm \pm}^{i}$ are listed in eq. (A.71) and $I_{ \pm \pm}^{i}$ in eq. (A.72).

As in the photon case, the exchange power corrections are non-zero only for transverse $\mu$ and $\nu$ in our approximation.

\subsection{Antisymmetric part of $Z$-boson hadronic tensor}

The antisymmetric part of hadronic tensor for cross section mediated by Z-boson is defined in eq. (2.9) where we should make substitution $\psi \rightarrow \Psi_{1}+\Psi_{2}$. Let us start from annihilationtype contribution

$$
\check{W}_{\mu \nu}^{\mathrm{ZAa}}(x)=\frac{N_{c}}{s}\left\langle A, B\left|\mathcal{J}_{12 \mu}(x) \mathcal{J}_{21 \nu}(0)-\mu \leftrightarrow \nu\right| A, B\right\rangle-x \leftrightarrow 0
$$

Using Fierz transformations (A.2) and (A.5) it can be rewritten as

$$
\check{W}_{\mu \nu}^{\mathrm{ZAa}}=\sum_{f} c_{f}^{2}\left[\frac{i}{2} \epsilon_{\mu \nu}^{\alpha \beta}\left[-2 a_{f} \check{\mathcal{W}}_{\alpha \beta}^{f \mathcal{F}}+\left(a_{f}^{2}+1\right) \check{\mathcal{W}}_{\alpha \beta}^{f 5}\right]+\left(a_{f}^{2}-1\right) \check{W}_{\mu \nu}^{\mathrm{as}}\right]
$$

where

$$
\begin{aligned}
\check{\mathcal{W}}_{\mu \nu}^{\mathcal{F}}(x)= & \frac{N_{c}}{2 s}\langle A, B|\left[\bar{\Psi}_{1}^{m}(x) \gamma_{\mu} \Psi_{1}^{n}(0)\right]\left[\bar{\Psi}_{2}^{n}(0) \gamma_{\nu} \Psi_{2}^{m}(x)\right] \\
& +\gamma_{\mu} \otimes \gamma_{\nu} \leftrightarrow \gamma_{\mu} \gamma_{5} \otimes \gamma_{\nu} \gamma_{5}-\mu \leftrightarrow \nu|A, B\rangle-x \leftrightarrow 0 \\
\check{\mathcal{W}}_{\mu \nu}^{5}(x)= & \frac{N_{c}}{2 s}\langle A, B|\left[\bar{\Psi}_{1}^{m}(x) \gamma_{\mu} \gamma_{5} \Psi_{1}^{n}(0)\right]\left[\bar{\Psi}_{2}^{n}(0) \gamma_{\nu} \Psi_{2}^{m}(x)\right] \\
& +\gamma_{\mu} \gamma_{5} \otimes \gamma_{\nu} \leftrightarrow \gamma_{\mu} \otimes \gamma_{\nu} \gamma_{5}-\mu \leftrightarrow \nu|A, B\rangle-x \leftrightarrow 0, \\
\check{W}_{\mu \nu}^{\mathrm{as}}(x)= & \frac{i N_{c}}{2 s}\langle A, B|-\left[\bar{\Psi}_{1}^{m}(x) \Psi_{1}^{n}(0)\right]\left[\bar{\Psi}_{2}^{n}(0) \sigma_{\mu \nu} \Psi_{2}^{m}(x)\right] \\
& +\left[\bar{\Psi}_{1}^{m}(x) \sigma_{\mu \nu} \Psi_{1}^{n}(0)\right]\left[\bar{\Psi}_{2}^{n}(0) \Psi_{2}^{m}(x)\right]+\left[\bar{\Psi}_{1}^{m}(x) \gamma_{5} \Psi_{1}^{n}(0)\right]\left[\bar{\Psi}_{2}^{n}(0) \sigma_{\mu \nu} \gamma_{5} \Psi_{2}^{m}(x)\right] \\
& \left.-\left[\bar{\Psi}_{1}^{m}(x) \sigma_{\mu \nu} \gamma_{5} \Psi_{1}^{n}(0)\right]\left[\bar{\Psi}_{2}^{n}(0) \gamma_{5} \Psi_{2}^{m}(x)\right]\right\}|A, B\rangle-x \leftrightarrow 0
\end{aligned}
$$

for the flavor under consideration.

Let us start from the $\mathcal{W}_{\mu \nu}^{\mathcal{F}}(x)$ given by the first line in eq. ((5.24)) and compare it to $W_{\mu \nu}(x)$ for the photon case. It is easy to see that if we take $W_{\mu \nu}^{\mathrm{F}}(x)$ and antisymmetrize with respect to $\mu$ and $\nu$ instead of symmetrization, we will get $\mathcal{W}_{\mu \nu}^{\mathcal{F}}(x)$. Since antisymmetrization $v s$ symmetrization does not affect power counting in $\frac{q_{\perp}^{2}}{s}$ parameter (or $\alpha_{q}, \beta_{q} \ll 1$ parameter), we can consider only terms that gave leading contribution to $W_{\mu \nu}(x)$. 
First, consider the leading-twist contribution ${ }^{9}$

$$
\begin{aligned}
\check{\mathcal{W}}_{\mu \nu}^{\mathcal{F}, \mathrm{lt}}(x)= & \frac{1}{2 s}\left(\left\langle\bar{\psi}\left(x_{\bullet}, x_{\perp}\right) \gamma_{\mu} \psi(0)\right\rangle_{A}\left\langle\bar{\psi}_{B}(0) \gamma_{\nu} \psi\left(x_{\star}, x_{\perp}\right)\right\rangle_{B}\right. \\
& \left.+\gamma_{\mu} \otimes \gamma_{\nu} \leftrightarrow \gamma_{\mu} \gamma_{5} \otimes \gamma_{\nu} \gamma_{5}-\mu \leftrightarrow \nu\right)-x \leftrightarrow 0 .
\end{aligned}
$$

Using parametrizations (A.39) and (A.42) we get

$$
\begin{aligned}
\mathcal{W}_{\mu \nu}^{\mathcal{F}, \mathrm{lt}}(q) & =\frac{1}{16 \pi^{4}} \int d x_{\bullet} d x_{\star} d^{2} x_{\perp} e^{-i \alpha_{q} x_{\bullet}-i \beta_{q} x_{\star}+i(q, x)_{\perp}} \check{\mathcal{W}}_{\mu \nu}^{\mathcal{F}, l t}(x) \\
& =-\frac{2}{s}\left(p_{1 \mu} p_{2 \nu}-p_{1 \nu} p_{2 \mu}\right) \int d^{2} k_{\perp} \mathcal{F}\left(q, k_{\perp}\right)
\end{aligned}
$$

where

$$
\mathcal{F}^{f}\left(q, k_{\perp}\right)=f_{1}^{f}\left(\alpha_{q}, k_{\perp}\right) \bar{f}_{1}^{f}\left(\beta_{q},(q-k)_{\perp}\right)-f_{1}^{f} \leftrightarrow \bar{f}_{1}^{f}
$$

As usually, term with $f_{1} \leftrightarrow \bar{f}_{1}$ comes from $x \leftrightarrow 0$ contribution.

Next, we consider terms with gluon operators and separate them as in section 4.1 according to number of gluon fields (contained in $\Xi$ 's):

$$
\check{\mathcal{W}}_{\mu \nu}^{\mathcal{F}}(x)=\check{\mathcal{W}}_{\mu \nu}^{\mathcal{F}, l t}+\check{\mathcal{W}}_{\mu \nu}^{1 \mathcal{F}}(x)+\check{\mathcal{W}}_{\mu \nu}^{(2 a) \mathcal{F}}+\check{\mathcal{W}}_{\mu \nu}^{(2 b) \mathcal{F}}+\check{\mathcal{W}}_{\mu \nu}^{(2 c) \mathcal{F}}
$$

where leading-twist term $\check{\mathcal{W}}_{\mu \nu}^{\mathcal{F}, l t}$ was considered above, and

$$
\begin{aligned}
\check{\mathcal{W}}_{\mu \nu}^{1 \mathcal{F}}(x)= & \frac{N_{c}}{2 s}\langle A, B|\left[\bar{\psi}_{A}^{m}(x) \gamma_{\mu} \Xi_{1}^{n}(0)\right]\left[\bar{\psi}_{B}^{n}(0) \gamma_{\nu} \psi_{B}^{m}(x)\right] \\
+ & {\left[\bar{\Xi}_{1}^{m}(x) \gamma_{\mu} \psi_{A}^{n}(0)\right]\left[\bar{\psi}_{B}^{n}(0) \gamma_{\nu} \psi_{A}^{m}(x)\right]+\left[\bar{\psi}_{A}^{m}(x) \gamma_{\mu} \psi_{A}^{n}(0)\right]\left[\bar{\psi}_{B}^{n}(0) \gamma_{\nu} \Xi_{2}^{m}(x)\right] } \\
+ & {\left[\bar{\psi}_{A}^{m}(x) \gamma_{\mu} \psi_{A}^{n}(0)\right]\left[\bar{\Xi}_{2}^{n}(0) \gamma_{\nu} \psi_{B}^{m}(x)\right]+\gamma_{\mu} \otimes \gamma_{\nu} \leftrightarrow \gamma_{\mu} \gamma_{5} \otimes \gamma_{\nu} \gamma_{5}-\mu \leftrightarrow \nu|A, B\rangle-x \leftrightarrow 0 } \\
\check{\mathcal{W}}_{\mu \nu}^{(2 a) \mathcal{F}}(x)= & \frac{N_{c}}{2 s}\langle A, B|\left[\bar{\psi}_{A}^{m}(x) \gamma_{\mu} \Xi_{1}^{n}(0)\right]\left[\bar{\psi}_{B}^{n}(0) \gamma_{\nu} \Xi_{2}^{m}(x)\right] \\
& +\left[\bar{\Xi}_{1}^{m}(x) \gamma_{\mu} \psi_{A}^{n}(0)\right]\left[\bar{\Xi}_{2}^{n}(0) \gamma_{\nu} \psi_{B}^{m}(x)\right]+\gamma_{\mu} \otimes \gamma_{\nu} \leftrightarrow \gamma_{\mu} \gamma_{5} \otimes \gamma_{\nu} \gamma_{5}-\mu \leftrightarrow \nu|A, B\rangle-x \leftrightarrow 0 \\
\check{\mathcal{W}}_{\mu \nu}^{(2 b) \mathcal{F}}(x)= & \frac{N_{c}}{2 s}\langle A, B|\left[\bar{\psi}_{A}^{m}(x) \gamma_{\mu} \psi_{A}^{n}(0)\right]\left[\bar{\Xi}_{2}^{n}(0) \gamma_{\nu} \Xi_{2}^{m}(x)\right] \\
& +\left[\bar{\Xi}_{1}^{m}(x) \gamma_{\mu} \Xi_{1}^{n}(0)\right]\left[\bar{\psi}_{B}^{n}(0) \gamma_{\nu} \psi_{B}^{m}(x)\right]+\gamma_{\mu} \otimes \gamma_{\nu} \leftrightarrow \gamma_{\mu} \gamma_{5} \otimes \gamma_{\nu} \gamma_{5}-\mu \leftrightarrow \nu|A, B\rangle-x \leftrightarrow 0
\end{aligned}
$$

and

$$
\begin{aligned}
\check{\mathcal{W}}_{\mu \nu}^{(2 c) \mathcal{F}}(x)= & \frac{N_{c}}{2 s}\langle A, B|\left[\bar{\Xi}_{1}^{m}(x) \gamma_{\mu} \psi_{A}^{n}(0)\right]\left[\bar{\psi}_{B}^{n}(0) \gamma_{\nu} \Xi_{2}^{m}(x)\right] \\
& +\left[\bar{\psi}_{A}^{m}(x) \gamma_{\mu} \Xi_{1}^{n}(0)\right]\left[\bar{\Xi}_{2}^{n}(0) \gamma_{\nu} \psi_{B}^{m}(x)\right]+\gamma_{\mu} \otimes \gamma_{\nu} \leftrightarrow \gamma_{\mu} \gamma_{5} \otimes \gamma_{\nu} \gamma_{5}-\mu \leftrightarrow \nu|A, B\rangle-x \leftrightarrow 0
\end{aligned}
$$

The corresponding contributions to $\mathcal{W}_{\mu \nu}(q)$ will be denoted $\mathcal{W}_{\mu \nu}^{(1) \mathcal{F}}, \mathcal{W}_{\mu \nu}^{(2 a) \mathcal{F}}, \mathcal{W}_{\mu \nu}^{(2 b) \mathcal{F}}$, and $\mathcal{W}_{\mu \nu}^{(2 c) \mathcal{F}}$, respectively. We will consider these contributions in turn following the analysis in section 4.1.

\footnotetext{
${ }^{9}$ Recall that "check" means $W$ 's in coordinate space multiplied by $\frac{2 N_{c}}{s}$, cf. eq. (4.1).
} 


\subsubsection{One-gluon terms in $\mathcal{W}_{\mu \nu}^{\mathcal{F}}$}

First, we consider terms with one gluon operator and start with

$\left[\bar{\psi}_{A}^{m}(x) \gamma_{\mu} \Xi_{1}^{n}(0)\right]\left[\bar{\psi}_{B}^{n}(0) \gamma_{\nu} \psi_{B}^{m}(x)\right]$. Using $\Xi_{1}=-\frac{\not p_{2}}{s} \gamma^{i} B_{i} \frac{1}{\alpha} \psi_{A}$ and separating colorsinglet terms, we get

$$
\begin{aligned}
\check{\mathcal{W}}_{1 \mu \nu}^{(1) \mathcal{F}}(x)= & -\frac{1}{2 s^{2}}\left\{\left\langle\bar{\psi}(x) \gamma_{\mu} \not p_{2} \gamma_{i} \frac{1}{\alpha} \psi(0)\right\rangle_{A}\left\langle\bar{\psi} B^{i}(0) \gamma_{\nu} \psi(x)\right\rangle_{B}\right. \\
& +\left(\psi(0) \otimes \psi(x) \leftrightarrow \gamma_{5} \psi(0) \otimes \gamma_{5} \psi(x)-\mu \leftrightarrow \nu\right\}-x \leftrightarrow 0
\end{aligned}
$$

As we discussed above, we need to consider only terms which gave leading contribution for symmetric case, i.e. with one index longitudinal and the other transverse. Similarly to eq. (4.14), for longitudinal $\mu$ and transverse $\nu$ we get

$$
\begin{aligned}
& \left(\frac{2 p_{1}^{\mu} p_{2}^{\mu^{\prime}}}{s}+\mu \leftrightarrow \mu^{\prime}\right) \check{\mathcal{W}}_{\mu \nu}^{(1) \mathcal{F}}(x) \\
& =-\left(\frac{p_{2 \mu} p_{1}^{\mu^{\prime}}}{s^{3}}+\mu \leftrightarrow \mu^{\prime}\right)\left\{\left\langle\bar{\psi}(x) \gamma_{\mu^{\prime}} \not_{2} \gamma_{i} \frac{1}{\alpha} \psi(0)\right\rangle_{A}\left\langle\bar{\psi} B^{i}(0) \gamma_{\nu_{\perp}} \psi(x)\right\rangle_{B}\right. \\
& \quad+\left(\psi(0) \otimes \psi(x) \leftrightarrow \gamma_{5} \psi(0) \otimes \gamma_{5} \psi(x)-\mu^{\prime} \leftrightarrow \nu\right\}-x \leftrightarrow 0
\end{aligned}
$$

The term proportional to $p_{2 \mu}$ in the r.h.s. can be expressed using eq. (A.20) as follows:

$$
\begin{aligned}
& \frac{p_{2 \mu}}{s^{3}}\left[\left\langle\bar{\psi}(x) \gamma_{\nu_{\perp}} \not \not_{2} \gamma_{i} \frac{1}{\alpha} \psi(0)\right\rangle_{A}\left\langle\bar{\psi} B^{i}(0) \not \not_{1} \psi(x)\right\rangle_{B}-\left\langle\bar{\psi}(x) \not p_{1} \not \not_{2} \gamma_{i} \frac{1}{\alpha} \psi(0)\right\rangle_{A}\left\langle\bar{\psi} B^{i}(0) \gamma_{\nu_{\perp}} \psi(x)\right\rangle_{B}\right. \\
& +\left(\psi(0) \otimes \psi(x) \leftrightarrow \gamma_{5} \psi(0) \otimes \gamma_{5} \psi(x)\right]-x \leftrightarrow 0 \\
& \quad=\frac{p_{2 \mu}}{s^{3}}\left[\left\langle\bar{\psi}(x) \not p_{2} \frac{1}{\alpha} \psi(0)\right\rangle_{A}\left\langle\bar{\psi}(0) \not B(0) \not \not_{1} \gamma_{\nu_{\perp}} \psi(x)\right\rangle_{B}\right. \\
& \left.\quad+\left(\psi(0) \otimes \psi(x) \leftrightarrow \gamma_{5} \psi(0) \otimes \gamma_{5} \psi(x)\right)\right]-x \leftrightarrow 0
\end{aligned}
$$

since the second term in the first line is $O\left(\frac{q_{\perp}^{2}}{s}\right)$ with respect to the first one. Next, if $\nu$ is longitudinal and $\mu$ transverse, we consider $\check{\mathscr{W}}_{1 \nu \mu}^{(1) F}=-\check{\mathcal{W}}_{1 \mu \nu}^{(1) F}$, repeat the above calculation and get result (5.35) with $\mu \leftrightarrow \nu$. Thus, the case with longitudinal $\nu$ and transverse $\mu$ is obtained from (5.35) by $-(\mu \leftrightarrow \nu)$ replacement, so using eqs. (A.45), (A.50) and parametrizations from section A.2 we obtain

$$
\mathcal{W}_{1 \mu \nu}^{(1) \mathcal{F}}(q)=\frac{p_{2 \mu}}{\alpha_{q} s} \int d^{2} k_{\perp}(q-k)_{\nu}^{\perp} \mathcal{F}\left(q, k_{\perp}\right)-\mu \leftrightarrow \nu
$$

which is the same as eq. (4.15), only with antisymmetrization in $\mu, \nu$ and $f \leftrightarrow \bar{f}$ instead of symmetrizaton. 
The second term in the r.h.s. of eq. (5.29) with longitudinal $\mu$ and transverse $\nu$ can be obtained in a similar way. Repeating steps from eq. (5.33) to eq. (5.35), we get

$$
\begin{aligned}
\check{\mathcal{W}}_{2 \mu \nu}^{(1) \mathcal{F}}(x)= & \frac{N_{c}}{2 s}\left\langle A, B\left|\left[\bar{\Xi}_{1}^{m}(x) \gamma_{\mu} \psi_{A}^{n}(0)\right]\left[\bar{\psi}_{B}^{n}(0) \gamma_{\nu} \psi_{A}^{m}(x)\right]\right| A, B\right\rangle \\
& +\gamma_{\mu} \otimes \gamma_{\nu} \leftrightarrow \gamma_{\mu} \gamma_{5} \otimes \gamma_{\nu} \gamma_{5}-\mu \leftrightarrow \nu|A, B\rangle-x \leftrightarrow 0 \\
= & -\frac{1}{2 s^{2}}\left\{\left\langle\left(\bar{\psi} \frac{1}{\alpha}\right)(x) \gamma_{i} \not p_{2} \gamma_{\mu} \frac{1}{\alpha} \psi(0)\right\rangle_{A}\left\langle\bar{\psi}(0) \gamma_{\nu} B^{i}(x) \psi(x)\right\rangle_{B}\right. \\
& +\left(\psi(0) \otimes \psi(x) \leftrightarrow \gamma_{5} \psi(0) \otimes \gamma_{5} \psi(x)-\mu \leftrightarrow \nu\right\}-x \leftrightarrow 0 \\
= & -\left(\frac{p_{2 \mu} p_{1}^{\mu^{\prime}}}{s^{3}}+\mu \leftrightarrow \mu^{\prime}\right)\left\{\left\langle\bar{\psi}(x) \gamma_{i} \not p_{2} \gamma_{\mu^{\prime}} \frac{1}{\alpha} \psi(0)\right\rangle_{A}\left\langle\bar{\psi}(0) \gamma_{\nu_{\perp}} B^{i}(x) \psi(x)\right\rangle_{B}\right. \\
& +\left(\psi(0) \otimes \psi(x) \leftrightarrow \gamma_{5} \psi(0) \otimes \gamma_{5} \psi(x)-\mu^{\prime} \leftrightarrow \nu\right\}-x \leftrightarrow 0 \\
\simeq & \frac{p_{2 \mu}}{s^{3}}\left[\left\langle\bar{\psi}(x) \not p_{2} \frac{1}{\alpha} \psi(0)\right\rangle_{A}\left\langle\bar{\psi}(0) \gamma_{\nu_{\perp}} \not p_{1} \not B(x) \psi(x)\right\rangle_{B}\right. \\
& \left.+\left(\psi(0) \otimes \psi(x) \leftrightarrow \gamma_{5} \psi(0) \otimes \gamma_{5} \psi(x)\right)\right]-x \leftrightarrow 0
\end{aligned}
$$

The opposite case with transverse $\mu$ and longitudinal $\nu$ is obtained by $-(\mu \leftrightarrow \nu)$ and therefore from eq. (A.53) we get

$$
\mathcal{W}_{2 \mu \nu}^{(1) \mathcal{F}}(q)=\frac{p_{2 \mu}}{\alpha_{q} s} \int d^{2} k_{\perp}(q-k)_{\nu}^{\perp} \mathcal{F}\left(q, k_{\perp}\right)-\mu \leftrightarrow \nu
$$

which doubles the result (5.36) similarly to the symmetric case.

Rewriting now the third term in the r.h.s. of eq. (5.29) with longitudinal $\mu$ and transverse $\nu$ and repeating the above steps, we get (recall $\Xi_{2}=-\frac{\not p}{s} \gamma^{i} A_{i} \frac{1}{\beta} \psi_{B}$ ):

$$
\begin{aligned}
\check{\mathcal{W}}_{1 \mu \nu}^{(2) \mathcal{F}}(x)= & \frac{N_{c}}{2 s}\langle A, B|\left[\bar{\psi}_{A}^{m}(x) \gamma_{\mu} \psi_{A}^{n}(0)\right]\left[\bar{\psi}_{B}^{n}(0) \gamma_{\nu} \Xi_{2}^{m}(x)\right] \\
& +\gamma_{\mu} \otimes \gamma_{\nu} \leftrightarrow \gamma_{\mu} \gamma_{5} \otimes \gamma_{\nu} \gamma_{5}-\mu \leftrightarrow \nu|A, B\rangle+x \leftrightarrow 0 \\
= & -\frac{1}{2 s^{2}}\left\{\left\langle\bar{\psi}(x) A^{i}(x) \gamma_{\mu} \psi(0)\right\rangle_{A}\left\langle\bar{\psi}(0) \gamma_{\nu} \not p_{1} \gamma_{i} \frac{1}{\beta} \psi(x)\right\rangle_{B}\right. \\
& +\left(\psi(0) \otimes \psi(x) \leftrightarrow \gamma_{5} \psi(0) \otimes \gamma_{5} \psi(x)-\mu \leftrightarrow \nu\right\}-x \leftrightarrow 0 \\
= & -\left(\frac{p_{1 \mu} p_{2}^{\mu^{\prime}}}{s^{3}}+\mu \leftrightarrow \mu^{\prime}\right)\left\{\left\langle\bar{\psi}(x) A^{i}(x) \gamma_{\mu^{\prime}} \psi(0)\right\rangle_{A}\left\langle\bar{\psi}(0) \gamma_{\nu} p_{1} \gamma_{i} \frac{1}{\beta} \psi(x)\right\rangle_{B}\right. \\
& +\left(\psi(0) \otimes \psi(x) \leftrightarrow \gamma_{5} \psi(0) \otimes \gamma_{5} \psi(x)-\mu^{\prime} \leftrightarrow \nu\right\}-x \leftrightarrow 0 \\
= & \frac{p_{1 \mu}}{s^{3}}\left\{\left\langle\bar{\psi}(x) \not p_{2} \breve{A}_{\nu_{\perp}}(x) \psi(0)\right\rangle_{A}\left\langle\bar{\psi}(0) \not p_{1} \frac{1}{\beta} \psi(x)\right\rangle_{B}\right. \\
& +\left(\psi(0) \otimes \psi(x) \leftrightarrow \gamma_{5} \psi(0) \otimes \gamma_{5} \psi(x)\right\}-x \leftrightarrow 0
\end{aligned}
$$

As above, the case with longitudinal $\nu$ and transverse $\mu$ is obtained from (5.39) by $-(\mu \leftrightarrow \nu)$ replacement, so using eqs. (A.45), (A.50) and parametrizations from section A.2 we obtain

$$
\mathcal{W}_{1 \mu \nu}^{(2) \mathcal{F}}(q)=-\frac{p_{1 \mu}}{\beta_{q} s} \int d^{2} k_{\perp} k_{\nu}^{\perp} \mathcal{F}\left(q, k_{\perp}\right)-\mu \leftrightarrow \nu
$$


Finally, similarly to eq. (5.37), it can be demonstrated that the forth term in the r.h.s. of eq. (5.29) $\left[\bar{\psi}_{A}^{m}(x) \gamma_{\mu} \psi_{A}^{n}(0)\right]\left[\bar{\Xi}_{2}^{n}(0) \gamma_{\nu} \psi_{B}^{m}(x)\right]$ doubles the contribution (5.40) of the third term, so we get

$$
\mathcal{W}_{\mu \nu}^{(1) \mathcal{F}}(q)=2 \int d^{2} k_{\perp}\left[\frac{p_{2 \mu}(q-k)_{\nu}^{\perp}}{\alpha_{q} s}-\frac{p_{1 \mu} k_{\nu}^{\perp}}{\beta_{q} s}\right] \mathcal{F}\left(q, k_{\perp}\right)-\mu \leftrightarrow \nu
$$

Note that it can be obtained from eq. (4.17) by replacement of symmetrization in $\mu \leftrightarrow \nu$ and $f \leftrightarrow \bar{f}$ with antisymmetrization.

\subsubsection{Two-gluon terms in $\mathcal{W}_{\mu \nu}^{\mathcal{F}}$}

We start from the first term in the r.h.s. of eq. (5.30)

$$
\begin{aligned}
\check{\mathcal{W}}_{1 \mu \nu}^{(2 a) \mathcal{F}}(x)= & \frac{N_{c}}{2 s}\langle A, B|\left[\bar{\psi}_{A}^{m}(x) \gamma_{\mu} \Xi_{1}^{n}(0)\right]\left[\bar{\psi}_{B}^{n}(0) \gamma_{\nu} \Xi_{2}^{m}(x)\right] \\
& +\gamma_{\mu} \otimes \gamma_{\nu} \leftrightarrow \gamma_{\mu} \gamma_{5} \otimes \gamma_{\nu} \gamma_{5}-\mu \leftrightarrow \nu|A, B\rangle-x \leftrightarrow 0
\end{aligned}
$$

Separating color-singlet contributions one can rewrite eq. (5.42) as

$$
\begin{aligned}
\check{\mathcal{W}}_{1 \mu \nu}^{(2 a) \mathcal{F}}(x)= & \frac{1}{2 s^{3}}\left\{\left\langle\bar{\psi} A_{i}(x) \gamma_{\mu} \not p_{2} \gamma^{j} \frac{1}{\alpha} \psi(0)\right\rangle_{A}\left\langle\bar{\psi} B_{j}(0) \gamma_{\nu} \not p_{1} \gamma^{i} \frac{1}{\beta} \psi(x)\right\rangle_{B}\right. \\
& \left.+\psi(0) \otimes \psi(x) \leftrightarrow \gamma_{5} \psi(0) \otimes \gamma_{5} \psi(x)-\mu \leftrightarrow \nu\right\}-x \leftrightarrow 0
\end{aligned}
$$

Similar to the symmetric case discussed in section 4.1.2, the leading term comes from $\mu$ and $\nu$ that are both transverse. In this case we can use formula (A.28) and get

$$
\begin{aligned}
\check{\mathcal{W}}_{1 \mu_{\perp} \nu_{\perp}}^{(2 a) \mathcal{F}}(x)= & \frac{1}{2 s^{3}}\left\{\left\langle\bar{\psi} A_{i}(x) \gamma_{\mu_{\perp}} \not p_{2} \gamma^{j} \frac{1}{\alpha} \psi(0)\right\rangle_{A}\left\langle\bar{\psi} B_{j}(0) \gamma_{\nu_{\perp}} \not p_{1} \gamma^{i} \frac{1}{\beta} \psi(x)\right\rangle_{B}\right. \\
& \left.+\psi(0) \otimes \psi(x) \leftrightarrow \gamma_{5} \psi(0) \otimes \gamma_{5} \psi(x)-\mu \leftrightarrow \nu\right\}-x \leftrightarrow 0 \\
= & -\frac{1}{s^{3}}\left(\left\langle\bar{\psi}(x) \not p_{2} \breve{A}_{\mu}(x) \frac{1}{\alpha} \psi(0)\right\rangle_{A}\left\langle\bar{\psi}(0) \not p_{1} \breve{B}_{\nu}(0) \frac{1}{\beta} \psi(x)\right\rangle_{B}-\mu \leftrightarrow \nu\right)-x \leftrightarrow 0
\end{aligned}
$$

Using now eqs. (A.45), (A.50) and (A.52) we obtain contribution to $\mathcal{W}_{\mu \nu}^{F}(q)$ in the form

$$
\begin{aligned}
\mathcal{W}_{1 \mu_{\perp} \nu_{\perp}}^{(2 a) \mathcal{F}}(x) & =\frac{1}{16 \pi^{4}} \int d x_{\bullet} d x_{\star} d^{2} x_{\perp} e^{-i \alpha_{q} x_{\bullet}-i \beta_{q} x_{\star}+i(q, x)_{\perp}} \check{\mathcal{W}}_{1 \mu_{\perp} \nu_{\perp}}^{(2 a) F}(x) \\
& =\frac{1}{Q_{\|}^{2}} \int d^{2} k_{\perp}\left[k_{\mu}^{\perp}(q-k)_{\nu}^{\perp}-\mu \leftrightarrow \nu\right] \mathcal{F}\left(q, k_{\perp}\right)=0 .
\end{aligned}
$$

This term vanishes after integration over $k_{\perp}$ but we will keep it for a while since we want to have gauge invariance at the integrand level, see eq. (5.51) below.

Next, second term in the r.h.s. of eq. (5.30)

$$
\begin{aligned}
\check{\mathcal{W}}_{2 \mu \nu}^{(2 a) \mathcal{F}}(x)= & \frac{N_{c}}{2 s}\langle A, B|\left[\bar{\Xi}_{1}^{m}(x) \gamma_{\mu} \psi_{A}^{n}(0)\right]\left[\bar{\Xi}_{2}^{n}(0) \gamma_{\nu} \psi_{B}^{m}(x)\right] \\
& +\gamma_{\mu} \otimes \gamma_{\nu} \leftrightarrow \gamma_{\mu} \gamma_{5} \otimes \gamma_{\nu} \gamma_{5}-\mu \leftrightarrow \nu|A, B\rangle-x \leftrightarrow 0
\end{aligned}
$$


at transverse $\mu$ and $\nu$ can be transformed to

$$
\begin{aligned}
\check{\mathcal{W}}_{2 \mu_{\perp} \nu_{\perp}}^{(2 a) \mathcal{F}}(x)= & \frac{1}{2 s^{3}}\left\{\left\langle\left(\bar{\psi} \frac{1}{\alpha}\right)(x) \gamma^{j} \not \not_{2} \gamma_{\mu_{\perp}} A_{i}(0) \psi(0)\right\rangle_{A}\left\langle\left(\bar{\psi} \frac{1}{\beta}\right)(0) \gamma^{i} \not p_{1} \gamma_{\nu_{\perp}} B_{j}(x) \psi(x)\right\rangle_{B}\right. \\
& \left.+\psi(0) \otimes \psi(x) \leftrightarrow \gamma_{5} \psi(0) \otimes \gamma_{5} \psi(x)-\mu \leftrightarrow \nu\right\}-x \leftrightarrow 0 \\
= & -\frac{1}{s^{3}}\left\{\left\langle\left(\bar{\psi} \frac{1}{\alpha}\right)(x) \breve{A}_{\mu_{\perp}}(0) \not \not_{2} \psi(0)\right\rangle_{A}\left\langle\left(\bar{\psi} \frac{1}{\beta}\right)(0) \breve{B}_{\nu_{\perp}}(x) \not p_{1} \psi(x)\right\rangle_{B}-\mu \leftrightarrow \nu\right\}-x \leftrightarrow 0
\end{aligned}
$$

where we used formula (A.28). It is easy to see that the corresponding contribution to $\mathcal{W}_{2 \mu_{\perp} \nu_{\perp}}^{(2 a) \mathcal{F}}(q)$ doubles the result (5.45), same as for eq. (4.22) in the symmetric case, and we obtain

$$
\mathcal{W}_{\mu_{\perp} \nu \perp}^{(2 a) \mathcal{F}}(x)=\frac{2}{Q_{\|}^{2}} \int d^{2} k_{\perp}\left[k_{\mu}^{\perp}(q-k)_{\nu}^{\perp}-\mu \leftrightarrow \nu\right] \mathcal{F}\left(q, k_{\perp}\right) .
$$

Again, this integral vanishes, but we keep the integrand as a part of eq. (5.51) in order to have gauge invariance (5.52) visible at the integrand level.

As one can anticipate from eq. (4.26) for the symmetric case, the contribution from eq. (5.31) vanishes. Indeed, e.g. for the first term we get

$$
\begin{aligned}
& \frac{N_{c}}{2 s}\left\langle A, B\left|\left[\bar{\psi}_{A}^{m}(x) \gamma_{\mu} \psi_{A}^{n}(0)\right]\left[\bar{\Xi}_{2}^{n}(0) \gamma_{\nu} \Xi_{2}^{m}(x)\right]+\gamma_{\mu} \otimes \gamma_{\nu} \leftrightarrow \gamma_{\mu} \gamma_{5} \otimes \gamma_{\nu} \gamma_{5}-\mu \leftrightarrow \nu\right| A, B\right\rangle \\
& =\frac{p_{1 \nu}}{s^{3}}\left(\left\langle\bar{\psi}(x) A_{j}(x) \gamma_{\mu} A_{i}(0) \psi(0)\right\rangle_{A}\left\langle\left(\bar{\psi} \frac{1}{\beta}\right)(0) \gamma^{i} \not p_{1} \gamma^{j} \frac{1}{\beta} \psi(x)\right\rangle_{B}\right. \\
& \left.\quad+\psi(0) \otimes \psi(x) \leftrightarrow \gamma_{5} \psi(0) \otimes \gamma_{5} \psi(x)\right)-\mu \leftrightarrow \nu
\end{aligned}
$$

Next, if the index $\mu$ is transverse, the contribution of this equation to $\mathcal{W}_{\mu \nu}^{\mathcal{F}}$ is of order of $p_{1 \nu} q_{\mu}^{\perp} \frac{m_{\perp}^{2}}{\beta_{q}^{2} s^{2}}$ (cf. eq. (4.24)) which is $O\left(\frac{q_{\perp}^{2}}{\beta_{q} s}\right)$ in comparison to eq. (5.41). Also, if index $\nu$ is longitudinal, the contribution is $\sim p_{1 \nu} p_{2 \mu} \frac{q_{\perp}^{4}}{\beta_{q}^{2} s^{3}}$ which is $O\left(\frac{\alpha_{q}^{2} m^{4}}{Q_{\|}^{4}}\right)$ in comparison to leading-twist result (5.26). Thus, $\check{\mathcal{W}}_{\mu \nu}^{(2 b) \mathcal{F}}(x)=0$ with our accuracy. Finally, as discussed in section 4.1.2, the term $\check{\mathcal{W}}_{\mu \nu}^{(2 c) \mathcal{F}}(x)$ is of order of $\frac{1}{N_{c}^{2}}$ and can be neglected.

\subsubsection{Sum of $\mathcal{W}_{\mu \nu}^{\mathcal{F}}$ terms}

Adding the contributions (5.26), (5.41), and (5.48) we obtain

$$
\mathcal{W}_{\mu \nu}^{\mathcal{F}}(q)=\int d^{2} k_{\perp} \mathcal{F}\left(q, k_{\perp}\right) \mathcal{W}_{\mu \nu}^{F}\left(q, k_{\perp}\right)
$$

where $\mathcal{F}^{f}\left(q, k_{\perp}\right)$ is given by eq. (5.27) and

$$
\begin{aligned}
\mathcal{W}_{\mu \nu}^{F}\left(q, k_{\perp}\right) & =\frac{2 p_{1 \nu} p_{2 \mu}}{s}+\frac{2 p_{2 \mu}(q-k)_{\nu}^{\perp}}{\alpha_{q} s}+\frac{2 p_{1 \nu} k_{\mu}^{\perp}}{\beta_{q} s}+\frac{2 k_{\mu}^{\perp}(q-k)_{\nu}^{\perp}}{\alpha_{q} \beta_{q} s}-\mu \leftrightarrow \nu \\
& =\frac{q_{\mu}}{Q_{\|}^{2}}\left(\tilde{q}_{\nu}+q_{\nu}^{\perp}-2 k_{\nu}^{\perp}\right)-\mu \leftrightarrow \nu
\end{aligned}
$$

The corresponding contribution to antisymmetric part of $Z$-boson hadronic tensor $\check{\mathcal{W}}_{\mu \nu}^{\text {asy }}(q)$ is proportional to $\epsilon_{\mu \nu \lambda \rho} \mathcal{W}^{\lambda \rho}(q)$ so we immediately see gauge invariance:

$$
q_{\mu} \epsilon^{\mu \nu \alpha \beta} \mathcal{W}_{\alpha \beta}^{F}\left(q, k_{\perp}\right)=0
$$




\subsection{4 $\mathcal{W}_{\mu \nu}^{\mathrm{f5}}$ contribution}

From eq. (5.24) we see that $\mathcal{W}_{\mu \nu}^{\mathcal{F}}$ and $\mathcal{W}_{\mu \nu}^{5}$ differ by replacement $\psi(0) \rightarrow \gamma_{5} \psi(0)$. Let us consider terms assembled in $\mathcal{W}_{\mu \nu}^{\mathcal{F}}$ and prove that they vanish after such replacement. Fist, for the leading-twist contribution it is evident from parametrizations (A.43). Second, let us write down

$$
\begin{aligned}
& \check{\mathcal{W}}_{1 \mu \nu}^{(1) \mathcal{F}}(x)+\check{\mathcal{W}}_{2 \mu \nu}^{(1) \mathcal{F}}(x)= \\
& =\frac{p_{1 \mu}}{s^{3}}\left\{\left\langle\bar{\psi}(x) \not p_{2} \frac{1}{\alpha} \psi(0)\right\rangle_{A}\left\langle\bar{\psi}(0) \not B(0) \not p_{1} \gamma_{\nu_{\perp}} \psi(x)\right\rangle_{B}+\left\langle\bar{\psi}(x) \not p_{2} \frac{1}{\alpha} \psi(0)\right\rangle_{A}\right. \\
& \quad \times\left\langle\bar{\psi}(0) \gamma_{\nu_{\perp}} \not p_{1} \not B(x) \psi(x)\right\rangle_{B}+\left(\psi(0) \otimes \psi(x) \leftrightarrow \gamma_{5} \psi(0) \otimes \gamma_{5} \psi(x)\right\}-x \leftrightarrow 0
\end{aligned}
$$

The contributions of these two terms are equal, but using equations from section A.3 it is easy to see that after replacement $\psi(0) \rightarrow \gamma_{5} \psi(0)$ they cancel each other as in the eq. (5.7) case so $\check{\mathscr{W}}_{1 \mu \nu}^{(1) 5}(x)=0$. Similarly, one can demonstrate that

$$
\begin{aligned}
\check{W}_{3 \mu \nu}^{(1) \mathrm{F} 5}(x)+\check{W}_{4 \mu \nu}^{(1) \mathrm{F} 5}(x)= & \frac{p_{1 \mu}}{s^{2}}\left[\left\langle\bar{\psi} \not A(x) \not \not_{2} \gamma_{\nu_{\perp}} \gamma_{5} \psi(0)\right\rangle_{A}\left\langle\bar{\psi}(0) \not p_{1} \frac{1}{\beta} \psi(x)\right\rangle_{B}\right. \\
& +\left\langle\bar{\psi}(x) \gamma_{\nu_{\perp}} \not p_{2} \not A(0) \gamma_{5} \psi(0)\right\rangle_{A}\left\langle\left(\bar{\psi} \frac{1}{\beta}\right)(0) \not p_{1} \psi(x)\right\rangle_{B} \\
& \left.+\psi(0) \otimes \psi(x) \leftrightarrow \psi(0) \gamma_{5} \otimes \gamma_{5} \psi(x)\right]+\mu \leftrightarrow \nu+x \leftrightarrow 0
\end{aligned}
$$

vanishes since the two terms in the r.h.s. cancel each other.

Let us turn now to terms with two gluon operators and start from $\check{\mathcal{W}}_{\mu \nu}^{(2 \mathrm{a}) \mathrm{F} 5}(x)$. The $\check{\mathcal{W}}_{\mu \nu}^{(2 a) \mathcal{F}}(x)$ contribution is given by sum of eqs. (5.44) and (5.47)

$$
\begin{aligned}
\check{\mathcal{W}}_{\mu \nu}^{(2 a) \mathcal{F}}(x)= & -\frac{1}{s^{3}}\left(\left\langle\bar{\psi}(x) \not p_{2} \breve{A}_{\mu}(x) \frac{1}{\alpha} \psi(0)\right\rangle_{A}\left\langle\bar{\psi}(0) \not \not_{1} \breve{B}_{\nu}(0) \frac{1}{\beta} \psi(x)\right\rangle_{B}\right. \\
& \left.\left.+\left(\bar{\psi} \frac{1}{\alpha}\right)(x) \breve{A}_{\mu_{\perp}}(0) \not p_{2} \psi(0)\right\rangle_{A}\left\langle\left(\bar{\psi} \frac{1}{\beta}\right)(0) \breve{B}_{\nu_{\perp}}(x) \not p_{1} \psi(x)\right\rangle_{B}\right)-\mu \leftrightarrow \nu-x \leftrightarrow 0
\end{aligned}
$$

As we saw in section 5.2.2, the contributions of the two terms in the r.h.s. are equal. Now, when we replace $\psi(0) \rightarrow \gamma_{5} \psi(0)$, target matrix elements terms remain the same and projectile ones become of different sign as seen from eq. (A.59) so $\check{\mathcal{W}}_{\mu \nu}^{(2 \mathrm{a}) \mathrm{F} 5}(x)=0$

Finally, the contribution $\check{\mathcal{W}}_{\mu \nu}^{(2 \mathrm{~b}) \mathrm{F} 5}(x)$ is small by power counting (see eq. (5.49) and subsequent discussion) while $\check{\mathcal{W}}_{\mu \nu}^{(2 \mathrm{c}) 5}(x)$ has extra $\frac{1}{N_{c}^{2}}$ so we neglect it. Thus, $\mathcal{W}_{\mu \nu}^{5}=0$ with our accuracy.

As to $W_{\mu \nu}^{\text {as }}$ defined in eq. (5.24), it also vanishes with our accuracy but the calculation is more tedious. 


\section{$5.3 W_{\mu \nu}^{\text {as }}$}

In this section we will prove that $W_{\mu \nu}^{\text {as }}$ defined in eq. (5.24) is small in our approximation. As usual, for power counting we consider $W_{\mu \nu}^{\text {as }}(x)$ multiplied by $2 N_{c} / s$ :

$$
\begin{aligned}
\check{W}_{\mu \nu}^{\text {as }}(x)= & \frac{i N_{c}}{2 s}\left\{\left[\bar{\Psi}_{1}^{m}(x) \sigma_{\mu \nu} \Psi_{1}^{n}(0)\right]\left[\bar{\Psi}_{2}^{n}(0) \Psi_{2}^{m}(x)\right]-\left[\bar{\Psi}_{1}^{m}(x) \Psi_{1}^{n}(0)\right]\left[\bar{\Psi}_{2}^{n}(0) \sigma_{\mu \nu} \Psi_{2}^{m}(x)\right]\right. \\
& \left.-\psi(0) \otimes \psi(x) \leftrightarrow \gamma_{5} \psi(0) \otimes \gamma_{5} \psi(x)\right\}-x \leftrightarrow 0
\end{aligned}
$$

First, from parametrizations in Sect A.2 it is clear that the leading-twist contribution to $\breve{W}_{\mu \nu}^{\text {as }}$ is of order of $\frac{p_{1 \mu} q_{\nu}^{\perp}}{s}$ (or $\frac{p_{2 \mu} q_{\nu}^{\perp}}{s}$ ) which is $O\left(\beta_{q}\right)$ (or $O\left(\alpha_{q}\right)$ ) with respect to contribution (5.51).

\subsubsection{One-gluon terms}

Next, consider terms with one gluon field and start from term with $\Xi_{1}=-\frac{\not p_{2}}{s} \gamma^{i} B_{i} \frac{1}{\alpha} \psi_{A}$. We get the contribution to the r.h.s. of eq. (5.56) in the form

$$
\begin{aligned}
& \frac{i}{s^{2}}\left\{\left\langle\bar{\psi}(x) \not p_{2} \gamma_{i} \frac{1}{\alpha} \psi(0)\right\rangle_{A}\left\langle\bar{\psi}(0) B^{i}(0) \sigma_{\mu \nu} \psi(x)\right\rangle_{B}\right. \\
& \left.-\left\langle\bar{\psi}(x) \sigma_{\mu \nu} \not \not_{2} \gamma^{i} \frac{1}{\alpha} \psi(0)\right\rangle_{A}\left\langle\bar{\psi}(0) B_{i}(0) \psi(x)\right\rangle_{B}-\psi(0) \otimes \psi(x) \leftrightarrow \gamma_{5} \psi(0) \otimes \gamma_{5} \psi(x)\right\}-x \leftrightarrow 0
\end{aligned}
$$

Let us first take transverse $\mu$ and $\nu$ and consider the first term in the r.h.s. Since the projectile matrix element $\left\langle\psi(x) \not p_{2} \gamma_{i} \frac{1}{\alpha} \psi(0)\right\rangle_{A}$ is proportional to $x_{i}$ and the target one

$\left\langle\psi(0) B^{i}(0) \sigma_{\mu \nu}^{\perp} \psi(x)\right\rangle_{B}$ to $\delta_{\mu}^{i} x_{\nu}^{\perp}-\delta_{\nu}^{i} x_{\mu}^{\perp}$, this term vanishes. Since $\sigma_{\mu \nu}^{\perp} \gamma^{i}=i\left(\delta_{\nu}^{i} \gamma_{\mu}^{\perp}-\right.$ $\left.\delta_{\mu}^{i} \gamma_{\nu}^{\perp}\right)$, the second term in the r.h.s. of eq. (5.57) vanishes for the same reason: target matrix element is proportional to $x_{i}$ and the projectile one to $\delta_{\mu}^{i} x_{\nu}^{\perp}-\delta_{\nu}^{i} x_{\mu}^{\perp}$. Next, consider terms with extra $\gamma_{5}$ 's. Since $\left\langle\psi(x) \not p_{2} \gamma_{i} \gamma_{5} \frac{1}{\alpha} \psi(0)\right\rangle_{A} \sim \epsilon_{i j} x^{j}$ and $\left\langle\psi(0) B^{i}(0) \sigma_{\mu \nu}^{\perp} \gamma_{5} \psi(x)\right\rangle_{B}=$ $\frac{2 i}{s} \epsilon_{\mu \nu}^{\perp}\left\langle\psi(0) B^{i}(0) \sigma_{\bullet \star}^{\perp} \gamma_{5} \psi(x)\right\rangle_{B} \sim x^{i}$, this term also gives no contribution. For the last term, since $\sigma_{\mu \nu}^{\perp} \gamma_{5}=i \epsilon_{\mu \nu}^{\perp} \frac{2}{s} \sigma_{\star \bullet}$, the projectile matrix elements proportional to $x^{i}$ and the target one to $\epsilon_{i j} x^{j}$ so the last term in the r.h.s. of eq. (5.57) vanishes. Now, terms with $\bar{\Xi}_{1}=$ $-\left(\bar{\psi}_{A} \frac{1}{\alpha}\right) \gamma^{i} B_{i} \frac{\not p_{2}}{s}$ are similar to that of eq. (5.57) so they vanish for the same reason. Finally, the results for $\bar{\Xi}_{2}$ and $\Xi_{2}$ differ by usual projectile $\leftrightarrow$ target replacements so we get the result that one-gluon contributions to $\check{W}_{\mu \nu}^{\text {as }}$ vanish at transverse $\mu$ and $\nu$.

If now both $\mu$ and $\nu$ are longitudinal, $\sigma_{\mu \nu}=\frac{4}{s^{2}}\left(p_{1 \mu} p_{2 \nu}-\mu \leftrightarrow \nu\right) \sigma_{\star \bullet}$. It is easy to see that $\sigma_{\star \bullet}$ in the target matrix element brings no factor of $s$ while in the projectile one $\sigma_{\star \bullet} \not p_{2} \gamma_{i}=s \sigma_{\bullet i}$ can bring $s^{2}$. However, even in this case the corresponding contribution to r.h.s. of eq. (5.57) is proportional to $\frac{2}{\alpha_{q} s^{2}}\left(p_{1 \mu} p_{2 \nu}-\mu \leftrightarrow \nu\right)=\frac{\beta_{q}}{q_{\|}^{2}} \times \frac{2}{s}\left(p_{1 \mu} p_{2 \nu}-\mu \leftrightarrow \nu\right)$ which is $O\left(\beta_{q}\right)$ smaller than the last term in eq. (5.51) $\sim \epsilon_{\mu \nu i j} \frac{k^{i}(q-k)^{j}}{q_{\|}^{2}} \sim \frac{2}{s}\left(p_{1 \mu} p_{2 \nu}-\mu \leftrightarrow \nu\right) \frac{q_{1}^{2}}{q^{2}}$. 
Finally, let us consider case when one of the indices is longitudinal and the other transverse. The corresponding contribution to $\check{W}_{\mu \nu}^{\text {as }}(x)$ is (cf. eq. (4.43))

$$
\begin{aligned}
& \frac{2 p_{2 \mu}}{s^{3}}\left\{\left\langle\bar{\psi}(x) \sigma_{\star i} \frac{1}{\alpha} \psi(0)\right\rangle_{A}\left\langle\bar{\psi}(0) B^{i}(0) \sigma_{\bullet \nu_{\perp}} \psi(x)\right\rangle_{B}-\left\langle\bar{\psi}(x) \sigma_{\bullet \nu_{\perp}} \sigma_{\star i} \frac{1}{\alpha} \psi(0)\right\rangle_{A}\right. \\
& \left.\quad \times\left\langle\bar{\psi}(0) B^{i}(0) \psi(x)\right\rangle_{B}-\psi(0) \otimes \psi(x) \leftrightarrow \gamma_{5} \psi(0) \otimes \gamma_{5} \psi(x)\right\}-\mu \leftrightarrow \nu-x \leftrightarrow 0
\end{aligned}
$$

Using formulas (A.9) it is easy to see that the second (and the fourth) term can be neglected since the corresponding contribution to $\check{W}_{\mu \nu}^{\text {as }}(q)$ is $\sim\left(p_{2 \mu} q_{\nu}^{\perp}-\mu \leftrightarrow \nu\right) \frac{q_{\perp}^{2}}{\alpha_{q} s^{2}}$ which is $O\left(\alpha_{q}\right)$ in comparison to the second term in the r.h.s. of eq. (5.51) $\sim \epsilon_{\nu j} \frac{p_{2 \mu} q^{j}}{\alpha_{q} s}-\mu \leftrightarrow \nu$ so we are left with

$$
\begin{aligned}
\frac{2 p_{2 \mu}}{s^{3}} & \left\{\left\langle\bar{\psi}(x) \sigma_{\star i} \frac{1}{\alpha} \psi(0)\right\rangle_{A}\left\langle\bar{\psi}(0) B^{i}(0) \sigma_{\bullet \nu_{\perp}} \psi(x)\right\rangle_{B}\right. \\
& \left.-\left\langle\bar{\psi}(x) \sigma_{\star i} \gamma_{5} \frac{1}{\alpha} \psi(0)\right\rangle_{A}\left\langle\bar{\psi}(0) B^{i}(0) \sigma_{\bullet \nu_{\perp}} \gamma_{5} \psi(x)\right\rangle_{B}\right\}-\mu \leftrightarrow \nu-x \leftrightarrow 0
\end{aligned}
$$

Next, from eq. (A.12) we get

$$
\begin{aligned}
\sigma_{\star i} & \otimes \sigma_{\bullet \nu_{\perp}}-\sigma_{\star i} \gamma_{5} \otimes \sigma_{\bullet \nu_{\perp}} \gamma_{5} \\
& =-g_{i \nu} \sigma_{\star l} \otimes \sigma_{\bullet}^{l}+\sigma_{\star \nu_{\perp}} \otimes \sigma_{\bullet i}+\sigma_{\star i} \otimes \sigma_{\bullet \nu_{\perp}}-\frac{s}{4} g_{i \nu} \sigma_{m n} \otimes \sigma^{m n}+\frac{s}{2} \sigma_{\nu_{\perp} l} \otimes \sigma_{i}^{l}
\end{aligned}
$$

The two last terms can bring only factor $s$ to eq. (5.59) while the first three bring $\sim s^{2}$ so we get

$$
\begin{aligned}
& \frac{2 p_{2 \mu}}{s^{3}}\left\{\left\langle\bar{\psi}(x) \sigma_{\star i} \frac{1}{\alpha} \psi(0)\right\rangle_{A}\left\langle\bar{\psi}(0)\left[B^{i}(0) \sigma_{\bullet \nu_{\perp}}-B_{\nu}(0) \sigma_{\bullet}^{i}\right] \psi(x)\right\rangle_{B}\right. \\
& \left.+\left\langle\bar{\psi}(x) \sigma_{\star \nu_{\perp}} \frac{1}{\alpha} \psi(0)\right\rangle_{A}\left\langle\bar{\psi}(0) B^{i}(0) \sigma_{\bullet i} \psi(x)\right\rangle_{B}\right\}-\mu \leftrightarrow \nu-x \leftrightarrow 0 \\
& \quad=-i\left\langle\bar{\psi}(x) \sigma_{\star \nu_{\perp}} \frac{1}{\alpha} \psi(0)\right\rangle_{A}\left\langle\bar{\psi}(0) \not B(0) \not p_{1} \psi(x)\right\rangle_{B}-\mu \leftrightarrow \nu-x \leftrightarrow 0
\end{aligned}
$$

where we used eq. (4.34).

Let us calculate now the corresponding contribution to r.h.s. of eq. (5.56) coming from $\bar{\Xi}_{1}(x)$ (see eq. (3.4)):

$$
\begin{aligned}
& \frac{i}{s^{2}}\left\{\left\langle\left(\bar{\psi}(x) \frac{1}{\alpha}\right) \gamma_{i} \not \not_{2} \psi(0)\right\rangle_{A}\left\langle\bar{\psi}(0) B^{i}(0) \sigma_{\mu \nu} \psi(x)\right\rangle_{B}\right. \\
& \left.\quad-\left\langle\left(\bar{\psi} \frac{1}{\alpha}\right)(x) \sigma_{\mu \nu} \gamma_{i} \not \not_{2} \psi(0)\right\rangle_{A}\left\langle\bar{\psi}(0) B_{i}(0) \psi(x)\right\rangle_{B}-\psi(0) \otimes \psi(x) \leftrightarrow \gamma_{5} \psi(0) \otimes \gamma_{5} \psi(x)\right\}-x \leftrightarrow 0
\end{aligned}
$$

The only difference from eq. (5.56) is the sign $\gamma_{i} \not p_{2}=-\not p_{2} \gamma^{i}$, replacement $B^{i}(0) \rightarrow B^{i}(x)$ and $\frac{1}{\alpha}$ acting on $\bar{\psi}$ instead of $\psi$ so we get

$$
\begin{aligned}
& -\frac{2 p_{2 \mu}}{s^{3}}\left\{\left\langle\left(\bar{\psi} \frac{1}{\alpha}\right)(x) \psi(0)\right\rangle_{A}\left\langle\bar{\psi}(0)\left[B^{i}(0) \sigma_{\bullet \nu_{\perp}}-B_{\nu}(0) \sigma_{\bullet}^{i}\right] \psi(x)\right\rangle_{B}\right. \\
& \left.+\left\langle\left(\bar{\psi} \frac{1}{\alpha}\right)(x) \sigma_{\star \nu_{\perp}} \psi(0)\right\rangle_{A}\left\langle\bar{\psi}(0) B^{i}(x) \sigma_{\bullet i} \psi(x)\right\rangle_{B}\right\}-\mu \leftrightarrow \nu-x \leftrightarrow 0 \\
& =-i\left\langle\left(\bar{\psi} \frac{1}{\alpha}\right)(x) \sigma_{\star \nu_{\perp}} \psi(0)\right\rangle_{A}\left\langle\bar{\psi}(0) \not p_{1} \not B(x) \psi(x)\right\rangle_{B}-\mu \leftrightarrow \nu-x \leftrightarrow 0
\end{aligned}
$$


and the sum of these contributions to $\check{W}_{\mu \nu}^{\text {as }}(x)$ takes the form

$$
\begin{aligned}
- & i\left(\left\langle\bar{\psi}(x) \sigma_{\star \nu_{\perp}} \frac{1}{\alpha} \psi(0)\right\rangle_{A}\left\langle\bar{\psi}(0) \not B(0) \not p_{1} \psi(x)\right\rangle_{B}\right. \\
& \left.+\left\langle\left(\bar{\psi} \frac{1}{\alpha}\right)(x) \sigma_{\star \nu_{\perp}} \psi(0)\right\rangle_{A}\left\langle\bar{\psi}(0) \not p_{1} \not B(x) \psi(x)\right\rangle_{B}\right)-\mu \leftrightarrow \nu-x \leftrightarrow 0
\end{aligned}
$$

Now, from eqs. (A.45), (A.46), and (A.58) it is easy to see that the corresponding contribution to $\check{W}_{\mu \nu}^{\text {as }}(q)$ vanishes due to cancellation between the two terms in the above equation. Similarly, one can demonstrate that one-gluon contributions to $\check{W}_{\mu \nu}^{\text {as }}(q)$ from $\Xi_{2}=-\frac{\not p_{1}}{s} \gamma^{i} A_{i} \frac{1}{\beta+i \epsilon} \psi_{B}$ and $\bar{\Xi}_{2}=-\left(\bar{\psi}_{B} \frac{1}{\beta-i \epsilon}\right) \gamma^{i} A_{i} \frac{\not p_{1}}{s}$ cancel.

\subsubsection{Two-gluon terms}

Following analysis in section 4.1.2, let us start with the contribution to the r.h.s. of eq. (5.56) coming from $\Xi_{1}$ and $\Xi_{2}$ (see eq. (3.4)).

$$
\begin{aligned}
& \frac{i}{2 s^{3}}\left\{\left\langle\bar{\psi} A^{j}(x) \sigma_{\star i} \frac{1}{\alpha} \psi(0)\right\rangle_{A}\left\langle\bar{\psi} B^{i}(0) \sigma_{\mu \nu} \sigma_{\bullet j} \frac{1}{\beta} \psi(x)\right\rangle_{B}-\left\langle\bar{\psi} A^{j}(x) \sigma_{\mu \nu} \sigma_{\star i} \frac{1}{\alpha} \psi(0)\right\rangle_{A}\right. \\
& \left.\quad \times\left\langle\bar{\psi} B^{i}(0) \sigma_{\bullet j} \frac{1}{\beta} \psi(x)\right\rangle_{B}-\psi(0) \otimes \psi(x) \leftrightarrow \gamma_{5} \psi(0) \otimes \gamma_{5} \psi(x)\right\}-x \leftrightarrow 0
\end{aligned}
$$

The r.h.s. of eq. (5.65) at transverse $\mu$ and $\nu$ due to eq. (A.9) can be rewritten as

$$
\begin{aligned}
\text { eq. }(5.65)= & \frac{1}{2 s^{3}}\left\{\left\langle\bar{\psi} A_{\mu}(x) \sigma_{\star i} \frac{1}{\alpha} \psi(0)\right\rangle_{A}\left\langle\bar{\psi} B^{i}(0) \sigma_{\bullet \nu_{\perp}} \frac{1}{\beta} \psi(x)\right\rangle_{B}-\left\langle\bar{\psi} A^{i}(x) \sigma_{\star \nu_{\perp}} \frac{1}{\alpha} \psi(0)\right\rangle_{A}\right. \\
& \left.\times\left\langle\bar{\psi} B_{\mu}(0) \sigma_{\bullet} i \frac{1}{\beta} \psi(x)\right\rangle_{B}-\psi(0) \otimes \psi(x) \leftrightarrow \gamma_{5} \psi(0) \otimes \gamma_{5} \psi(x)\right\}-\mu \leftrightarrow \nu-x \leftrightarrow 0 .
\end{aligned}
$$

Using now eq. (A.35) we get

$$
\begin{aligned}
\text { eq. }(5.65)= & \frac{1}{2 s^{3}}\left\{\left\langle\bar{\psi} A_{\mu}(x) \sigma_{\star i} \frac{1}{\alpha} \psi(0)\right\rangle_{A}\left\langle\bar{\psi}\left[B^{i}(0) \sigma_{\bullet \nu_{\perp}}-B_{\nu}(0) \sigma_{\bullet}^{i}\right] \frac{1}{\beta} \psi(x)\right\rangle_{B}\right. \\
& +\left\langle\bar{\psi} A_{\mu}(x) \sigma_{\star \nu_{\perp}} \frac{1}{\alpha} \psi(0)\right\rangle_{A}\left\langle\bar{\psi} B^{i}(0) \sigma_{\bullet i} \frac{1}{\beta} \psi(x)\right\rangle_{B} \\
& +\left\langle\bar{\psi}\left[A_{\nu}(x) \sigma_{\star i}-A^{i}(x) \sigma_{\star \nu_{\perp}}\right] \frac{1}{\alpha} \psi(0)\right\rangle_{A}\left\langle\bar{\psi} B_{\mu}(0) \sigma_{\bullet}{ }^{i} \frac{1}{\beta} \psi(x)\right\rangle_{B} \\
& \left.-\left\langle\bar{\psi} A^{i}(x) \sigma_{\star i} \frac{1}{\alpha} \psi(0)\right\rangle_{A}\left\langle\bar{\psi} B_{\mu}(0) \sigma_{\bullet \nu_{\perp}} \frac{1}{\beta} \psi(x)\right\rangle_{B}\right\}-\mu \leftrightarrow \nu-x \leftrightarrow 0 \\
= & \frac{1}{2 s^{3}}\left\{\left\langle\bar{\psi}\left[A_{\mu}(x) \sigma_{\star \nu_{\perp}}-\mu \leftrightarrow \nu\right] \frac{1}{\alpha} \psi(0)\right\rangle_{A}\left\langle\bar{\psi} B^{i}(0) \sigma_{\bullet i} \frac{1}{\beta} \psi(x)\right\rangle_{B}\right. \\
& \left.-\left\langle\bar{\psi} A^{i}(x) \sigma_{\star i} \frac{1}{\alpha} \psi(0)\right\rangle_{A}\left\langle\bar{\psi}\left[B_{\mu}(0) \sigma_{\bullet \nu_{\perp}}-\mu \leftrightarrow \nu\right] \frac{1}{\beta} \psi(x)\right\rangle_{B}\right\}-x \leftrightarrow 0=0
\end{aligned}
$$


where we used eq. (4.34). Next, the contribution to the r.h.s. of eq. (5.56) coming from $\bar{\Xi}_{1}$ and $\bar{\Xi}_{2}$ has the form

$$
\begin{aligned}
& \frac{i}{2 s^{3}}\left\{\left\langle\left(\bar{\psi} \frac{1}{\alpha}\right)(x) \sigma_{\star i} A^{j} \psi(0)\right\rangle_{A}\left\langle\left(\bar{\psi} \frac{1}{\beta}\right)(0) \sigma_{\bullet j} \sigma_{\mu \nu} B^{i} \psi(x)\right\rangle_{B}-\left\langle\left(\bar{\psi} \frac{1}{\alpha}\right)(x) \sigma_{\star i} \sigma_{\mu \nu} A^{j}(0) \psi(0)\right\rangle_{A}\right. \\
& \left.\quad \times\left\langle\left(\bar{\psi} \frac{1}{\beta}\right)(0) \sigma_{\bullet j} B^{i} \psi(x)\right\rangle_{B}-\psi(0) \otimes \psi(x) \leftrightarrow \gamma_{5} \psi(0) \otimes \gamma_{5} \psi(x)\right\}-x \leftrightarrow 0
\end{aligned}
$$

and vanishes for transverse $\mu$ and $\nu$ for the same reason as the r.h.s. of eq. (5.67).

If both $\mu$ and $\nu$ are longitudinal, we get contribution to $\check{W}_{\mu \nu}^{\text {as }}(x)$ in the form

$$
\begin{aligned}
\text { eq. }(5.65)= & \frac{2 i}{s^{5}}\left(p_{1 \mu} p_{2 \nu}-\mu \leftrightarrow \nu\right)\left\{\left\langle\bar{\psi} A^{j}(x) \sigma_{\star i} \frac{1}{\alpha} \psi(0)\right\rangle_{A}\left\langle\bar{\psi} B^{i}(0) \sigma_{\star \bullet} \sigma_{\bullet j} \frac{1}{\beta} \psi(x)\right\rangle_{B}\right. \\
& \left.-\left\langle\bar{\psi} A^{j}(x) \sigma_{\star \bullet} \sigma_{\star i} \frac{1}{\alpha} \psi(0)\right\rangle_{A}\left\langle\bar{\psi} B^{i}(0) \sigma_{\bullet j} \frac{1}{\beta} \psi(x)\right\rangle_{B}-\psi(0) \otimes \psi(x) \leftrightarrow \gamma_{5} \psi(0) \otimes \gamma_{5} \psi(x)\right\} \\
& -x \leftrightarrow 0=\frac{2}{s^{4}}\left(p_{1 \mu} p_{2 \nu}-\mu \leftrightarrow \nu\right)\left\{\left\langle\bar{\psi} A^{j}(x) \sigma_{\star i} \frac{1}{\alpha} \psi(0)\right\rangle_{A}\left\langle\bar{\psi} B^{i}(0) \sigma_{\bullet j} \frac{1}{\beta} \psi(x)\right\rangle_{B}\right. \\
& \left.-\psi(0) \otimes \psi(x) \leftrightarrow \gamma_{5} \psi(0) \otimes \gamma_{5} \psi(x)\right\}-x \leftrightarrow 0 \\
= & \frac{2}{s^{4}}\left(p_{1 \mu} p_{2 \nu}-\mu \leftrightarrow \nu\right)\left\{\left\langle\bar{\psi} A^{i}(x) \sigma_{\star i} \frac{1}{\alpha} \psi(0)\right\rangle_{A}\left\langle\bar{\psi} B^{j}(0) \sigma_{\bullet} \frac{1}{\beta} \psi(x)\right\rangle_{B}\right. \\
& +\left\langle\bar{\psi} A^{j}(x) \sigma_{\star i} \frac{1}{\alpha} \psi(0)\right\rangle_{A}\left\langle\bar{\psi}\left[B^{i}(0) \sigma_{\bullet j}-B^{j}(0) \sigma_{\bullet i} \frac{1}{\beta} \psi(x)\right\rangle_{B}\right\}-x \leftrightarrow 0 \\
= & -\frac{2}{s^{4}}\left(p_{1 \mu} p_{2 \nu}-\mu \leftrightarrow \nu\right)\left\langle\bar{\psi} A(x) \not \not_{2} \frac{1}{\alpha} \psi(0)\right\rangle_{A}\left\langle\bar{\psi} \not B(0) \not p_{1} \frac{1}{\beta} \psi(x)\right\rangle_{B}-x \leftrightarrow 0
\end{aligned}
$$

where again we used eq. (A.35) and eq. (4.34). Similarly, the corresponding term in eq. (5.68) is

$$
\begin{aligned}
\text { eq. }(5.68)= & \frac{2 i}{s^{5}}\left(p_{1 \mu} p_{2 \nu}-\mu \leftrightarrow \nu\right)\left\{\left\langle\left(\bar{\psi} \frac{1}{\alpha}\right)(x) \sigma_{\star i} A^{j} \psi(0)\right\rangle_{A}\left\langle\left(\bar{\psi} \frac{1}{\beta}\right)(0) \sigma_{\bullet j} \sigma_{\star \bullet} B^{i} \psi(x)\right\rangle_{B}\right. \\
& -\left\langle\left(\bar{\psi} \frac{1}{\alpha}\right)(x) \sigma_{\star i} \sigma_{\star \bullet} A^{j} \psi(0)\right\rangle_{A}\left\langle\left(\bar{\psi} \frac{1}{\beta}\right)(0) \sigma_{\bullet j} B^{i} \psi(x)\right\rangle_{B} \\
& \left.-\psi(0) \otimes \psi(x) \leftrightarrow \gamma_{5} \psi(0) \otimes \gamma_{5} \psi(x)\right\}-x \leftrightarrow 0 \\
= & -\frac{2}{s^{4}}\left(p_{1 \mu} p_{2 \nu}-\mu \leftrightarrow \nu\right)\left\{\left\langle\left(\bar{\psi} \frac{1}{\alpha}\right)(0) \sigma_{\star i} A^{j} \psi(0)\right\rangle_{A}\left\langle\left(\bar{\psi} \frac{1}{\beta}\right)(0) \sigma_{\bullet j} B^{i} \psi(x)\right\rangle_{B}\right. \\
& \left.-\psi(0) \otimes \psi(x) \leftrightarrow \gamma_{5} \psi(0) \otimes \gamma_{5} \psi(x)\right\}-x \leftrightarrow 0 \\
= & -\frac{2}{s^{4}}\left(p_{1 \mu} p_{2 \nu}-\mu \leftrightarrow \nu\right)\left\{\left\langle\left(\bar{\psi} \frac{1}{\alpha}\right)(0) \sigma_{\star i} \frac{1}{\alpha} A^{i} \psi(0)\right\rangle_{A}\left\langle\left(\bar{\psi} \frac{1}{\beta}\right)(0) \sigma_{\bullet j} B^{j} \psi(x)\right\rangle_{B}\right. \\
& \left.+\left\langle\left(\bar{\psi} \frac{1}{\alpha}\right)(0) \sigma_{\star i} A^{j} \psi(0)\right\rangle_{A}\left\langle\left(\bar{\psi} \frac{1}{\beta}\right)(0)\left[B^{i}(x) \sigma_{\bullet j}-B^{j}(x) \sigma_{\bullet i}\right] \psi(x)\right\rangle_{B}\right\}-x \leftrightarrow 0 \\
= & -\frac{2}{s^{4}}\left(p_{1 \mu} p_{2 \nu}-\mu \leftrightarrow \nu\right)\left\langle\bar{\psi} A^{i}(x) \sigma_{\star i} \frac{1}{\alpha} \psi(0)\right\rangle_{A}\left\langle\bar{\psi} B^{j}(0) \sigma_{\bullet j} \frac{1}{\beta} \psi(x)\right\rangle_{B}-x \leftrightarrow 0 \\
= & \frac{2}{s^{4}}\left(p_{1 \mu} p_{2 \nu}-\mu \leftrightarrow \nu\right)\left\{\left\langle\left(\bar{\psi} \frac{1}{\alpha}\right)(0) \not p_{2} A \psi(0)\right\rangle_{A}\left\langle\left(\bar{\psi} \frac{1}{\beta}\right)(0) \not p_{1} \not B \psi(x)\right\rangle_{B}\right.
\end{aligned}
$$


Using QCD equations of motion from section A.3 we see that the contributions (5.69) and (5.70) cancel.

Now suppose $\mu$ is longitudinal and $\nu$ transverse

$$
\begin{aligned}
& \frac{i}{s^{4}}\left\{p_{1 \mu}\left\langle\bar{\psi} A^{j}(x) \sigma_{\star i} \frac{1}{\alpha} \psi(0)\right\rangle_{A}\left\langle\bar{\psi} B^{i}(0) \sigma_{\star \nu_{\perp}} \sigma_{\bullet j} \frac{1}{\beta} \psi(x)\right\rangle_{B}-p_{2 \mu}\left\langle\bar{\psi} A^{j}(x) \sigma_{\bullet \nu_{\perp}} \sigma_{\star i} \frac{1}{\alpha} \psi(0)\right\rangle_{A}\right. \\
& \left.\quad \times\left\langle\bar{\psi} B^{i}(0) \sigma_{\bullet j} \frac{1}{\beta} \psi(x)\right\rangle_{B}-\psi(0) \otimes \psi(x) \leftrightarrow \gamma_{5} \psi(0) \otimes \gamma_{5} \psi(x)\right\}-x \leftrightarrow 0
\end{aligned}
$$

Due to the first line in eq. (A.9), both projectile and target matrix elements can bring factor $s$ each, so the result is either $\sim p_{1 \mu} q_{\nu}^{\perp} \frac{q_{\perp}^{2}}{\alpha_{q} \beta_{q} s^{2}}$ or $\sim p_{2 \mu} q_{\nu}^{\perp} \frac{q_{\perp}^{2}}{\alpha_{q} \beta_{q} s^{2}}$, both of which are small in comparison to eq. (5.41).

Let us now consider term coming from $\bar{\Xi}_{1}$ and $\Xi_{1}$ :

$$
\begin{aligned}
\check{W}_{\mu \nu}^{\mathrm{as}}(x)= & \frac{i}{2 s^{3}}\left\{\left\langle\left(\bar{\psi} \frac{1}{\alpha}\right)(x) \gamma_{i} \not_{2} \sigma_{\mu \nu} \not p_{2} \gamma_{j} \frac{1}{\alpha} \psi(0)\right\rangle_{A}\left\langle\bar{\psi} B^{j}(0) B^{i} \psi(x)\right\rangle_{B}\right. \\
& \left.-\psi(0) \otimes \psi(x) \leftrightarrow \gamma_{5} \psi(0) \otimes \gamma_{5} \psi(x)\right\}-x \leftrightarrow 0 \\
= & \frac{i}{s^{3}} p_{2 \nu}\left\{\left\langle\left(\bar{\psi} \frac{1}{\alpha}\right)(x) \gamma_{i} \sigma_{\star \mu} \gamma_{j} \frac{1}{\alpha} \psi(0)\right\rangle_{A}\left\langle\bar{\psi} B^{j}(0) B^{i} \psi(x)\right\rangle_{B}\right. \\
& \left.-\psi(0) \otimes \psi(x) \leftrightarrow \gamma_{5} \psi(0) \otimes \gamma_{5} \psi(x)\right\}-\mu \leftrightarrow \nu-x \leftrightarrow 0
\end{aligned}
$$

Since nonzero contribution comes from transverse $\mu$, power counting for the r.h.s. of this equation gives $\frac{p_{2 \nu} q_{\mu}^{\perp}}{\alpha_{q}^{2} s^{2}}$ (see eq. (A.46)) which is $O\left(\frac{q_{\perp}^{2}}{\alpha_{q} s}\right)$ in comparison eq. (5.41). Similarly, the contribution to $\check{W}_{\mu \nu}^{\text {as }}(x)$ coming from $\bar{\Xi}_{2}$ and $\Xi_{2}$ will be $\sim \frac{p_{1 \nu} q_{\mu}^{\perp}}{\beta_{q}^{2} s^{2}}$ and hence negligible. Thus, we proved that $W_{\mu \nu}^{\text {as }}=0$ with our accuracy.

\subsubsection{Exchange-type power corrections to $W_{\mu \nu}^{\mathrm{ZA}}$}

Power corrections of the "exchange" type come from the terms

$$
\begin{aligned}
\left(\check{W}_{f f^{\prime}}^{\mathrm{ZA}}\right)_{\mu \nu}^{\mathrm{ex}}(x)= & \frac{N_{c}}{s}\left\langle A, B\left|\mathcal{J}_{1 \mu}(x) \mathcal{J}_{2 \nu}(0)-\mu \leftrightarrow \nu\right| A, B\right\rangle-x \leftrightarrow 0 \\
= & \sum_{f, f^{\prime}} c_{f} c_{f^{\prime}}\left(a_{f} a_{f^{\prime}}\left(\check{W}^{\mathrm{A} f f^{\prime}}\right)_{\mu \nu}^{\mathrm{ex}}(x)+\left(\check{W}_{55}^{\mathrm{A} f f^{\prime}}\right)_{\mu \nu}^{\mathrm{ex}}(x)-a_{f}\left(\check{W}_{5 \mathrm{a}}^{\mathrm{A} f f^{\prime}}\right)_{\mu \nu}^{\mathrm{ex}}(x)\right. \\
& \left.-a_{f^{\prime}}\left(\check{W}_{5 \mathrm{~b}}^{\mathrm{A} f f^{\prime}}\right)_{\mu \nu}^{\mathrm{ex}}(x)\right)
\end{aligned}
$$


where

$$
\begin{aligned}
& \left(\check{W}^{\mathrm{A}}\right)_{\mu \nu}^{\mathrm{ex}}(x)=\frac{N_{c}}{s}\left\langle A, B\left|\left[\bar{\Psi}_{1}(x) \gamma_{\mu} \Psi_{1}^{f}(x)\right]\left[\bar{\Psi}_{2}(0) \gamma_{\nu} \Psi_{2}^{f^{\prime}}(0)\right]-\mu \leftrightarrow \nu\right| A, B\right\rangle-x \leftrightarrow 0, \\
& \left(\check{W}_{55}^{\mathrm{A}}\right)_{\mu \nu}^{\mathrm{ex}}(x) \equiv \frac{N_{c}}{s}\left\langle A, B\left|\left[\bar{\Psi}_{1}(x) \gamma_{\mu} \gamma_{5} \Psi_{1}(x)\right]\left[\bar{\Psi}_{2}(0) \gamma_{\nu} \gamma_{5} \Psi_{2}(0)\right]-\mu \leftrightarrow \nu\right| A, B\right\rangle-x \leftrightarrow 0, \\
& \left(\check{W}_{5 \mathrm{a}}^{\mathrm{A}}\right)_{\mu \nu}^{\mathrm{ex}}(x) \equiv \frac{N_{c}}{s}\left\langle A, B\left|\left[\bar{\Psi}_{1}(x) \gamma_{\mu} \gamma_{5} \Psi_{1}(x)\right]\left[\bar{\Psi}_{2}(0) \gamma_{\nu} \Psi_{2}(0)\right]-\mu \leftrightarrow \nu\right| A, B\right\rangle-x \leftrightarrow 0, \\
& \left(\check{W}_{5 \mathrm{~b}}^{\mathrm{A}}\right)_{\mu \nu}^{\mathrm{ex}}(x) \equiv \frac{N_{c}}{s}\left\langle A, B\left|\left[\bar{\Psi}_{1}(x) \gamma_{\mu} \Psi_{1}(x)\right]\left[\bar{\Psi}_{2}(0) \gamma_{\nu} \gamma_{5} \Psi_{2}(0)\right]-\mu \leftrightarrow \nu\right| A, B\right\rangle-x \leftrightarrow 0
\end{aligned}
$$

To avoid cluttering formulas, we will omit trivial flavor indices until final result.

Let us start from $\left(W^{\mathrm{A}}\right)_{\mu \nu}^{\mathrm{ex}}(x)$. Since replacement of symmetrization in $\mu, \nu$ by antisymmetrizaton does not change power counting, we can start from analog of formula (4.71)

$$
\begin{aligned}
\left(\check{W}^{\mathrm{A}}\right)_{\mu \nu}^{\mathrm{ex}}(x)= & \frac{2 N_{c}}{s}\left\langle p_{A}, p_{B}\right|\left[\bar{\Xi}_{1}(x) \gamma_{\mu} \psi_{A}(x)+\bar{\psi}_{A}(x) \gamma_{\mu} \Xi_{1}(x)\right] \\
& \times\left[\bar{\Xi}_{2}(0) \gamma_{\nu} \psi_{B}(0)+\bar{\psi}_{B}(0) \gamma_{\nu} \Xi_{2}(0)\right]\left|p_{A}, p_{B}\right\rangle-\mu \leftrightarrow \nu-x \leftrightarrow 0 \\
= & \frac{N_{c}}{\left(N_{c}^{2}-1\right) s^{3}}\left(\left\langle\psi(x) \gamma_{\mu} \not p_{2} \gamma^{j} A_{k}(0) \frac{1}{\alpha} \psi(x)+\left(\bar{\psi} \frac{1}{\alpha}\right)(x) \gamma^{j} \not p_{2} \gamma_{\mu} A_{k}(0) \psi(x)\right\rangle_{A}\right. \\
& \left.\times\left\langle\bar{\psi}(0) \gamma_{\nu} \not p_{1} \gamma^{k} B_{j}(x) \frac{1}{\beta} \psi(0)\right\rangle_{B}+\left(\bar{\psi} \frac{1}{\beta}\right)(0) \gamma^{k} \not p_{1} \gamma_{\nu} B_{j}(x) \psi_{B}(0)\right\rangle_{B}-\mu \leftrightarrow \nu-x \leftrightarrow 0
\end{aligned}
$$

with transverse $\mu$ and $\nu$. Using eq. (A.33) we get

$$
\begin{aligned}
\left(\check{W}^{\mathrm{A}}\right)_{\mu \nu}^{\mathrm{ex}}(x)= & -\frac{N_{c}}{\left(N_{c}^{2}-1\right) s^{3}}\left\langle\left(\bar{\psi} \frac{1}{\alpha}\right)(x) \breve{A}_{\mu}(0) \not p_{2} \psi(x)+\bar{\psi}(x) \not p_{2} \breve{A}_{\mu}(0) \frac{1}{\alpha} \psi(x)\right\rangle_{A} \\
& \times\left\langle\left(\bar{\psi} \frac{1}{\beta}\right)(0) \breve{B}_{\nu}(x) \not p_{1} \psi(0)+\bar{\psi}(0) \not p_{1} \breve{B}_{\nu}(x) \frac{1}{\beta} \psi(0)\right\rangle_{B}-\mu \leftrightarrow \nu-x \leftrightarrow 0
\end{aligned}
$$

and therefore

$$
\begin{aligned}
\left(W^{A}\right)_{\mu \nu}^{\mathrm{ex}}(q)= & -\frac{N_{c}}{\left(N_{c}^{2}-1\right) Q_{\|}^{2}} \int d^{2} k_{\perp}\left[k_{\mu}^{\perp}(q-k)_{\nu}^{\perp}-\mu \leftrightarrow \nu\right] \\
& \times\left[\left(j_{2}-\bar{j}_{2}\right)\left(\alpha_{q}, k_{\perp}\right)\left(j_{2}^{*}-\bar{j}_{2}^{*}\right)\left(\beta_{q},(q-k)_{\perp}\right)-\text { c.c. }\right]
\end{aligned}
$$

where we used eqs. (A.66) and (A.67). Since the functions $j_{i}\left(x, k_{\perp}\right)$ are actually functions of $x$ and $k_{\perp}^{2}$, the r.h.s. of the above equationcan be proportional only to $g_{\mu \nu}^{\perp}$ or $q_{\mu}^{\perp} q_{\nu}^{\perp}$ and hence it vanishes

$$
\left(W^{A}\right)_{\mu \nu}^{\mathrm{ex}}(q)=0
$$

Similarly to the symmetric case studied in section 5.1.1, the replacement $\psi \rightarrow \gamma_{5} \psi$ in the projectile matrix elements leads to $k_{\mu} j_{1}, \rightarrow \pm i \epsilon_{\mu \nu} k^{\nu} j_{1,2}$ and the replacement $\psi \rightarrow \gamma_{5} \psi$ in target matrix elements yields $(q-k)_{\mu} j_{1,2} \rightarrow \pm i \epsilon_{\mu}(q-k)^{\nu} j_{1,2}$. Looking at the result (5.77) 
and taking care of signs of replacements $\psi \rightarrow \gamma_{5} \psi$ in parametrizations (A.66) and (A.70), we obtain

$$
\begin{aligned}
\left(\check{W}_{55}^{\mathrm{A} f f^{\prime}}\right)_{\mu \nu}^{\mathrm{ex}}(x)= & -\frac{N_{c}}{\left(N_{c}^{2}-1\right) s^{3}}\left(\left\langle\left(\bar{\psi} \frac{1}{\alpha}\right)(x) \breve{A}_{\mu}(0) \not p_{2} \gamma_{5} \psi(x)+\bar{\psi}(x) \not p_{2} \breve{A}_{\mu}(0) \frac{1}{\alpha} \gamma_{5} \psi(x)\right\rangle_{A}^{f}\right. \\
& \left.\times\left\langle\left(\bar{\psi} \frac{1}{\beta}\right)(0) \breve{B}_{\nu}(x) \not p_{1} \gamma_{5} \psi(0)\right\rangle_{B}+\bar{\psi}(0) \not p_{1} \breve{B}_{\nu}(x) \frac{1}{\beta} \gamma_{5} \psi(0)\right\rangle_{B}^{f^{\prime}}-\mu \leftrightarrow \nu-x \leftrightarrow 0 \\
\Rightarrow & \left(W_{\mu \nu}^{\mathrm{A} f f^{\prime}}\right)_{55}^{\mathrm{ex}}(q)=-\frac{N_{c}}{\left(N_{c}^{2}-1\right) Q_{\|}^{2}} \int d^{2} k_{\perp}\left[k_{\mu}^{\perp}(q-k)_{\nu}^{\perp}-\mu \leftrightarrow \nu\right] \\
& \times\left[\left(j_{2}+\bar{j}_{2}\right)^{f}\left(\alpha_{q}, k_{\perp}\right)\left(j_{2}^{*}+\bar{j}_{2}^{*}\right)^{f^{\prime}}\left(\beta_{q},(q-k)_{\perp}\right)-\text { c.c. }\right]=0
\end{aligned}
$$

for the same reason as eq. (5.78) above. The non-vanishing contributions come from

$$
\begin{aligned}
\left(\check{W}_{5 \mathrm{a}}^{\mathrm{A} f f^{\prime}}\right)_{\mu \nu}^{\mathrm{ex}}(x)= & -\frac{N_{c}}{\left(N_{c}^{2}-1\right) s^{3}}\left(\left\langle\left(\bar{\psi} \frac{1}{\alpha}\right)(x) \breve{A}_{\mu}(0) \not p_{2} \gamma_{5} \psi(x)+\bar{\psi}(x) \not p_{2} \breve{A}_{\mu}(0) \frac{1}{\alpha} \gamma_{5} \psi(x)\right\rangle_{A}^{f}\right. \\
& \left.\times\left\langle\left(\bar{\psi} \frac{1}{\beta}\right)(0) \breve{B}_{\nu}(x) \not p_{1} \psi(0)\right\rangle_{B}+\bar{\psi}(0) \not p_{1} \breve{B}_{\nu}(x) \frac{1}{\beta} \psi(0)\right\rangle_{B}^{f^{\prime}}-\mu \leftrightarrow \nu-x \leftrightarrow 0
\end{aligned}
$$

and

$$
\begin{aligned}
\left(\check{\mathcal{W}}_{5 \mathrm{~b}}^{\mathrm{A} f f^{\prime}}\right)_{\mu \nu}^{\mathrm{ex}}(x)= & -\frac{N_{c}}{\left(N_{c}^{2}-1\right) s^{3}}\left(\left\langle\left(\bar{\psi} \frac{1}{\alpha}\right)(x) \breve{A}_{\mu}(0) \not p_{2} \psi(x)+\bar{\psi}(x) \not p_{2} \breve{A}_{\mu}(0) \frac{1}{\alpha} \psi(x)\right\rangle_{A}^{f}\right. \\
& \left.\times\left\langle\left(\bar{\psi} \frac{1}{\beta}\right)(0) \breve{B}_{\nu}(x) \not p_{1} \gamma_{5} \psi(0)\right\rangle_{B}+\bar{\psi}(0) \not p_{1} \breve{B}_{\nu}(x) \gamma_{5} \frac{1}{\beta} \psi(0)\right\rangle_{B}^{f^{\prime}}-\mu \leftrightarrow \nu-x \leftrightarrow 0
\end{aligned}
$$

Similarly to eq. (5.79), from parametrizations (A.66) and (A.70) one obtains

$$
\begin{aligned}
& \left(W_{\mu \nu}^{\mathrm{A} f f^{\prime}}\right)_{5 \mathrm{a}}^{\mathrm{ex}}(q)=-\frac{i N_{c}}{\left(N_{c}^{2}-1\right) Q_{\|}^{2}} \int d^{2} k_{\perp}\left[\epsilon_{\mu m} k^{m}(q-k)_{\nu}-\mu \leftrightarrow \nu\right] J_{+-}^{2 f f^{\prime}}\left(q, k_{\perp}\right), \\
& \left(W_{\mu \nu}^{\mathrm{A} f f^{\prime}}\right)_{5 \mathrm{~b}}^{\mathrm{ex}}(q)=\frac{i N_{c}}{\left(N_{c}^{2}-1\right) Q_{\|}^{2}} \int d^{2} k_{\perp}\left[k_{\mu} \epsilon_{\nu n}(q-k)^{n}-\mu \leftrightarrow \nu\right] J_{-+}^{2 f f^{\prime}}\left(q, k_{\perp}\right)
\end{aligned}
$$

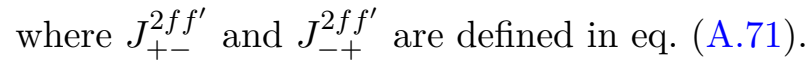

\subsection{Results for antisymmetric hadronic tensor for Z-mediated DY process}

The "annihilation" part is given by eqs. (5.23) and (5.51)

$$
W_{\mu \nu}^{\mathrm{ZAan}}(q)=-2 i \epsilon_{\mu \nu \alpha \beta} \frac{q^{\alpha}}{Q_{\|}^{2}} \int d^{2} k_{\perp}\left(\tilde{q}^{\beta}+q_{\perp}^{\beta}-2 k_{\perp}^{\beta}\right) \sum_{f} a_{f} c_{f}^{2} \mathcal{F}^{f}\left(q, k_{\perp}\right)
$$

It trivially satisfies $q^{\mu} W_{\mu \nu}^{\text {ZAan }}(q)=0$. 
The "exchange" part is given by the sum of contributions (5.73) calculated in previous section

$$
\begin{array}{rr}
W_{\mu \nu}^{\mathrm{ZAex}}(q) & \sum_{f, f^{\prime}} c_{f} c_{f^{\prime}}\left(W_{f f^{\prime}}^{\mathrm{ZA}}\right)_{\mu \nu}^{\mathrm{ex}}(q) \\
\left(W_{f f^{\prime}}^{\mathrm{ZA}}\right)_{\mu \nu}^{\mathrm{ex}}(q)= & \frac{i N_{c}}{\left(N_{c}^{2}-1\right) Q_{\|}^{2}} \int d^{2} k_{\perp}\left[a_{f}\left[\epsilon_{\mu m} k^{m}(q-k)_{\nu}-\mu \leftrightarrow \nu\right] J_{+-}^{2 f f^{\prime}}\left(q, k_{\perp}\right)\right. \\
\left.-a_{f^{\prime}}\left[k_{\mu} \epsilon_{\nu n}(q-k)^{n}-\mu \leftrightarrow \nu\right] J_{-+}^{2 f f^{\prime}}\left(q, k_{\perp}\right)\right]
\end{array}
$$

where $J_{+-}^{2 f f^{\prime}}$ and $J_{-+}^{2 f f^{\prime}}$ are defined in eq. (A.71).

It can be parametrized as

$$
\begin{aligned}
W_{\mu \nu}^{\mathrm{ZAex}}(q)= & \frac{i N_{c}}{\left(N_{c}^{2}-1\right) Q_{\|}^{2}} \sum_{f, f^{\prime}} c_{f} c_{f^{\prime}} \\
& \times\left[\left(\epsilon_{\mu \nu}+\frac{\epsilon_{\mu m} q^{m} q_{\nu}^{\perp}-\mu \leftrightarrow \nu}{q_{\perp}^{2}}\right)\left(a_{f} E_{+-}^{2 f f^{\prime}}+a_{f^{\prime}} E_{-+}^{2 f f^{\prime}}\right)-\epsilon_{\mu \nu}\left(a_{f} \mathcal{E}_{+-}^{2 f f^{\prime}}+a_{f^{\prime}} \mathcal{E}_{-+}^{2 f f^{\prime}}\right)\right]
\end{aligned}
$$

As usually, the exchange part is non-zero in our approximation only for transverse indices.

\section{Hadronic tensors for interference terms}

\subsection{Symmetric part of interference hadronic tensor $W^{\mathrm{I} 1}$}

From definitions (2.6) and (2.9) we get

$$
\begin{aligned}
\check{W}_{\mu \nu}^{\mathrm{I1S}}(x)= & \frac{N_{c}}{2 s} \sum_{f, f^{\prime}}\langle A, B|\left(e_{f^{\prime}} c_{f} a_{f}+e_{f} c_{f^{\prime}} a_{f^{\prime}}\right)\left[\bar{\psi}(x) \gamma_{\mu} \psi(x)\right]^{f}\left[\bar{\psi}(0) \gamma_{\nu} \psi(0)\right]^{f^{\prime}} \\
& -e_{f} c_{f^{\prime}}\left[\bar{\psi}(x) \gamma_{\mu} \psi(x)\right]^{f}\left[\bar{\psi}(0) \gamma_{\nu} \gamma_{5} \psi(0)\right]^{f^{\prime}} \\
& -e_{f^{\prime}} c_{f}\left[\bar{\psi}(x) \gamma_{\mu} \gamma_{5} \psi(x)\right]^{f}\left[\bar{\psi}(0) \gamma_{\nu} \psi(0)\right]^{f^{\prime}}|A, B\rangle+\mu \leftrightarrow \nu
\end{aligned}
$$

where $\psi=\Psi_{1}+\Psi_{2}$ in our approximation.

\subsubsection{Annihilation-type terms}

Let us start from the "annihilation" part

$$
\begin{aligned}
& \check{W}_{\mu \nu}^{\mathrm{I} 1 \mathrm{San}}(x)=\frac{N_{c}}{2 s} \sum_{f} e_{f} c_{f}\langle A, B| 2 a_{f}\left[\bar{\Psi}_{1}^{f} \gamma_{\mu} \Psi_{2}^{f}(x)\right]\left[\bar{\Psi}_{2}^{f} \gamma_{\nu} \Psi_{1}^{f}(0)\right] \\
& \quad-\left[\bar{\Psi}_{1}^{f} \gamma_{\mu} \Psi_{2}^{f}(x)\right]\left[\bar{\Psi}_{2}^{f} \gamma_{\nu} \gamma_{5} \Psi_{1}^{f}(0)\right]-\left[\bar{\Psi}_{1}^{f} \gamma_{\mu} \gamma_{5} \Psi_{2}^{f}(x)\right]\left[\bar{\Psi}_{2}^{f} \gamma_{\nu} \Psi_{1}^{f}(0)\right]|A, B\rangle+\mu \leftrightarrow \nu+x \leftrightarrow 0
\end{aligned}
$$

The first term can be copied from photon case (4.5) so we need to consider the last two terms. Using Fierz transformation (A.3) we get

$$
\begin{aligned}
& \frac{N_{c}}{2 s}\left\langle A, B\left|\left[\bar{\Psi}_{1} \gamma_{\mu} \Psi_{2}(x)\right]\left[\bar{\Psi}_{2} \gamma_{\nu} \gamma_{5} \Psi(0)\right]+\left[\bar{\Psi}_{1} \gamma_{\mu} \gamma_{5} \Psi_{2}(x)\right]\left[\bar{\Psi}_{2} \gamma_{\nu} \Psi_{1}(0)\right]\right| A, B\right\rangle+\mu \leftrightarrow \nu+x \leftrightarrow 0 \\
& =\left(g_{\mu \nu} g^{\alpha \beta}-\delta_{\mu}^{\alpha} \delta_{\nu}^{\beta}-\delta_{\nu}^{\alpha} \delta_{\mu}^{\beta}\right) \frac{N_{c}}{2 s}\langle A, B|\left[\bar{\Psi}_{1}(x) \gamma_{\alpha} \Psi_{1}(0)\right]\left[\bar{\Psi}_{2}(0) \gamma_{\nu} \gamma_{5} \Psi_{2}(x)\right] \\
& \quad+\left[\bar{\Psi}_{1}(x) \gamma_{\alpha} \gamma_{5} \Psi_{1}(0)\right]\left[\bar{\Psi}_{2}(0) \gamma_{\nu} \Psi_{2}(x)\right]|A, B\rangle+x \leftrightarrow 0=\check{W}_{\mu \nu}^{5}(x)
\end{aligned}
$$


Since we proved in section 5.1 that $\check{W}_{\mu \nu}^{5}$ is negligible (see eq. (5.5)) we get

$$
\begin{aligned}
\check{W}_{\mu \nu}^{\mathrm{I} 1 \mathrm{San}}(x) & =\sum_{f} e_{f} c_{f} a_{f} \check{W}^{f}(x) \\
& \Rightarrow W_{\mu \nu}^{\mathrm{I} 1 \mathrm{San}}(q)=\sum_{f} e_{f} c_{f} a_{f}\left[W_{\mu \nu}^{\mathrm{F} f}(q)+W_{\mu \nu}^{\mathrm{H} f}(q)+W_{\mu \nu}^{\mathrm{H} 2 f}(q)\right]
\end{aligned}
$$

where $W_{\mu \nu}^{\mathrm{F} f}, W_{\mu \nu}^{\mathrm{H} f}$, and $W_{\mu \nu}^{\mathrm{H} 2 f}$ are given by eqs. (4.30), (4.66), and (4.68), respectively.

\subsubsection{Exchange-type power corrections}

Let us consider now the exchange-type power corrections. From eq. (6.13) we get

$$
\begin{aligned}
\check{W}_{\mu \nu}^{\mathrm{I} 1 \mathrm{Sex}}(x)= & \frac{N_{c}}{2 s} \sum_{f}\langle A, B|\left(e_{f^{\prime}} c_{f} a_{f}+e_{f} c_{f^{\prime}} a_{f^{\prime}}\right)\left[\bar{\Psi}_{1}^{f} \gamma_{\mu} \Psi_{1}^{f}(x)\right]\left[\bar{\Psi}_{2}^{f^{\prime}} \gamma_{\nu} \Psi_{2}^{f^{\prime}}(0)\right] \\
& -e_{f} c_{f^{\prime}}\left[\bar{\Psi}_{1}^{f} \gamma_{\mu} \Psi_{1}^{f}(x)\right]\left[\bar{\Psi}_{2}^{f^{\prime}} \gamma_{\nu} \gamma_{5} \Psi_{2}^{f^{\prime}}(0)\right] \\
& -c_{f} e_{f^{\prime}}\left[\bar{\Psi}_{1}^{f} \gamma_{\mu} \gamma_{5} \Psi_{1}^{f}(x)\right]\left[\bar{\Psi}_{2}^{f^{\prime}} \gamma_{\nu} \Psi_{2}^{f^{\prime}}(0)\right]|A, B\rangle+\mu \leftrightarrow \nu+x \leftrightarrow 0 \\
= & \sum_{f, f^{\prime}}\left(\frac{1}{2}\left(e_{f^{\prime}} c_{f} a_{f}+e_{f} c_{f^{\prime}} a_{f^{\prime}}\right) \check{W}_{\mu \nu}^{f f^{\prime} \mathrm{ex}}(x)-\frac{e_{f^{\prime}} c_{f}}{2}\left(\check{W}_{5 \mathrm{a}}^{\mathrm{S} f f^{\prime}}\right)_{\mu \nu}^{\mathrm{ex}}(x)-\frac{e_{f} c_{f^{\prime}}}{2}\left(\check{W}_{5 \mathrm{~b}}^{\mathrm{S} f f^{\prime}}\right)_{\mu \nu}^{\mathrm{ex}}(x)\right)
\end{aligned}
$$

where $\check{W}_{\mu \nu}^{f f^{\prime} \mathrm{ex}}(x)$ is defined by eq. (4.71) while $\left(\check{W}_{5 \mathrm{a}, \mathrm{b}}^{\mathrm{S} f f^{\prime}}\right)_{\mu \nu}^{\mathrm{ex}}(x)$ are defined in eq. (5.12). Thus,

$$
\begin{aligned}
W_{\mu \nu}^{\mathrm{I} 1 \mathrm{Sex}}(q)=\sum_{f, f^{\prime}}\left[\frac{e_{f^{\prime}} c_{f} a_{f}+e_{f} c_{f^{\prime}} a_{f^{\prime}}}{2} W_{\mu \nu}^{f f^{\prime} \mathrm{ex}}(q)-\frac{e_{f^{\prime}} c_{f}}{2}\left(W_{5 \mathrm{a}}^{\mathrm{S} f f^{\prime}}\right)_{\mu \nu}^{\mathrm{ex}}(q)-\frac{e_{f} c_{f^{\prime}}}{2}\left(W_{5 \mathrm{~b}}^{\mathrm{S} f f^{\prime}}\right)_{\mu \nu}^{\mathrm{ex}}(q)\right] \\
=\frac{N_{c}}{2\left(N_{c}^{2}-1\right) Q_{\|}^{2}} \int d^{2} k_{\perp} \sum_{f, f^{\prime}}\left[( e _ { f ^ { \prime } } c _ { f } a _ { f } + e _ { f } c _ { f ^ { \prime } } a _ { f ^ { \prime } } ) \left(\left[k_{\mu}^{\perp}(q-k)_{\nu}^{\perp}+\mu \leftrightarrow \nu+g_{\mu \nu}^{\perp}(k, q-k)_{\perp}\right]\right.\right. \\
\left.\quad \times J_{--}^{1 f f^{\prime}}\left(q, k_{\perp}\right)-g_{\mu \nu}^{\perp}(k, q-k)_{\perp} J_{--}^{2 f f^{\prime}}\left(q, k_{\perp}\right)\right) \\
\quad-e_{f^{\prime}} c_{f}\left[\epsilon_{\mu m} k^{m}(q-k)_{\nu}+\mu \leftrightarrow \nu\right] I_{+-}^{1 f f^{\prime}}\left(q, k_{\perp}\right)+e_{f} c_{f^{\prime}}\left[k_{\mu} \epsilon_{\nu n}(q-k)^{n}+\mu \leftrightarrow \nu\right] I_{-+}^{1 f f^{\prime}}\left(q, k_{\perp}\right)
\end{aligned}
$$

where $W_{\mu \nu}^{f f^{\prime} \mathrm{ex}}(q),\left(W_{5 \mathrm{a}}^{\mathrm{S} f f^{\prime}}\right)_{\mu \nu}^{\mathrm{ex}}(q)$, and $\left(W_{5 \mathrm{~b}}^{\mathrm{S} f f^{\prime}}\right)_{\mu \nu}^{\mathrm{ex}}(q)$ are given in eqs. (4.80), (5.14), and (5.15), respectively.

\subsubsection{The result for the symmetric part of $W_{\mu \nu}^{\mathrm{I} 1}(x)$}

The result for $W_{\mu \nu}^{\mathrm{I1S}}(q)$ can be represented as a sum of "annihilation" and "exchange" parts

$$
\begin{aligned}
W_{\mu \nu}^{\mathrm{I} 1 \mathrm{~S}}(q) & =W_{\mu \nu}^{\mathrm{I} 1 \operatorname{San}}(q)+W_{\mu \nu}^{\mathrm{I} 1 \operatorname{Sex}}(q) \\
W_{\mu \nu}^{\mathrm{I} 1 \operatorname{San}}(q) & =\sum_{f} e_{f} c_{f} a_{f}\left[W_{\mu \nu}^{\mathrm{Ff}}(q)+W_{\mu \nu}^{\mathrm{Hf}}(q)+W_{\mu \nu}^{\mathrm{H} 2 \mathrm{f}}(q)\right] \\
W_{\mu \nu}^{\mathrm{I} 1 \mathrm{Sex}}(q) & =\text { r.h.s. of eq. }(6.5)
\end{aligned}
$$

where $W_{\mu \nu}^{\mathrm{Ff}}(q)$ is given by eq. (4.30), $W_{\mu \nu}^{\mathrm{Hf}}(q)$ by eq. (4.65), and $W_{\mu \nu}^{\mathrm{H} 2 \mathrm{f}}(q)$ by eq. (4.68). 


\subsection{Antiymmetric part of interference hadronic tensor $W^{\mathrm{I} 1}$}

From definitions (2.6) and (2.9) we get

$$
\begin{aligned}
\check{W}_{\mu \nu}^{\mathrm{I1} \mathrm{A}}(x)= & \frac{N_{c}}{2 s} \sum_{f, f^{\prime}}\langle A, B|\left(e_{f^{\prime}} c_{f} a_{f}+e_{f} c_{f^{\prime}} a_{f^{\prime}}\right)\left[\bar{\psi}(x) \gamma_{\mu} \psi(x)\right]^{f}\left[\bar{\psi}(0) \gamma_{\nu} \psi(0)\right]^{f^{\prime}} \\
& -e_{f} c_{f^{\prime}}\left[\bar{\psi}(x) \gamma_{\mu} \psi(x)\right]^{f}\left[\bar{\psi}(0) \gamma_{\nu} \gamma_{5} \psi(0)\right]^{f^{\prime}} \\
& -e_{f^{\prime}} c_{f}\left[\bar{\psi}(x) \gamma_{\mu} \gamma_{5} \psi(x)\right]^{f}\left[\bar{\psi}(0) \gamma_{\nu} \psi(0)\right]^{f^{\prime}}|A, B\rangle-\mu \leftrightarrow \nu
\end{aligned}
$$

where $\psi=\Psi_{1}+\Psi_{2}$ in our approximation.

Let us start from the annihilation part. After Fierz transformations (A.1) and (A.5) we get

$$
\begin{aligned}
\check{W}_{\mu \nu}^{\mathrm{I} 1 \mathrm{Aan}}(x)= & \frac{N_{c}}{2 s} \sum_{f} e_{f} c_{f}\langle A, B| 2 a_{f}\left[\bar{\Psi}_{1}^{f} \gamma_{\mu} \Psi_{2}^{f}(x)\right]\left[\bar{\Psi}_{2}^{f} \gamma_{\nu} \Psi_{1}^{f}(0)\right] \\
& -\left[\bar{\Psi}_{1}^{f} \gamma_{\mu} \Psi_{2}^{f}(x)\right]\left[\bar{\Psi}_{2}^{f} \gamma_{\nu} \gamma_{5} \Psi_{1}^{f}(0)\right]-\left[\bar{\Psi}_{1}^{f} \gamma_{\mu} \gamma_{5} \Psi_{2}^{f}(x)\right]\left[\bar{\Psi}_{2}^{f} \gamma_{\nu} \Psi_{1}^{f}(0)\right]|A, B\rangle \\
& -\mu \leftrightarrow \nu-x \leftrightarrow 0 \\
= & \sum_{f} e_{f} c_{f}\left[i a_{f} \epsilon_{\mu \nu}^{\alpha \beta} \check{\mathcal{W}}_{\alpha \beta}^{5 f}(x)+a_{f} \check{W}_{\mu \nu}^{\mathrm{as} f}(x)-\frac{i}{2} \epsilon_{\mu \nu}^{\alpha \beta} \check{\mathcal{W}}_{\alpha \beta}^{\mathcal{F} f}(x)\right]
\end{aligned}
$$

where we used definitions (5.24). As demonstrated in section 5.2, $\check{\mathcal{W}}_{\mu \nu}^{5 f}=\check{W}_{\mu \nu}^{\operatorname{as} f}=0$ with our accuracy and $\mathcal{W}_{\alpha \beta}^{\mathcal{F} f}(q)$ is given by eq. (5.50) so we obtain

$$
W_{\mu \nu}^{\mathrm{IIAan}}(q)=-i \epsilon_{\mu \nu \alpha \beta} q^{\alpha} \int d^{2} k_{\perp}\left(\tilde{q}+q_{\perp}-2 k_{\perp}\right)^{\beta} \sum_{f} e_{f} c_{f} \mathcal{F}^{f}\left(q, k_{\perp}\right)
$$

The exchange-type power corrections are

$$
\begin{gathered}
\check{W}_{\mu \nu}^{\mathrm{I} A \mathrm{Aex}}(x)=\frac{N_{c}}{2 s} \sum_{f, f^{\prime}}\langle A, B|\left(e_{f^{\prime}} c_{f} a_{f}+e_{f} c_{f^{\prime}} a_{f^{\prime}}\right)\left[\bar{\Psi}_{1}(x) \gamma_{\mu} \Psi_{1}(x)\right]^{f}\left[\bar{\Psi}_{2}(0) \gamma_{\nu} \Psi_{2}(0)\right]^{f^{\prime}} \\
-e_{f} c_{f^{\prime}}\left[\bar{\Psi}_{1}(x) \gamma_{\mu} \Psi_{1}(x)\right]^{f}\left[\bar{\Psi}_{2}(0) \gamma_{\nu} \gamma_{5} \Psi_{2}(0)\right]^{f^{\prime}} \\
-e_{f^{\prime}} c_{f}\left[\bar{\Psi}_{1}(x) \gamma_{\mu} \gamma_{5} \Psi_{1}(x)\right]^{f}\left[\bar{\Psi}_{2}(0) \gamma_{\nu} \bar{\Psi}_{2}(0)\right]^{f^{\prime}}|A, B\rangle-\mu \leftrightarrow \nu-x \leftrightarrow 0 \\
=\sum_{f, f^{\prime}}\left[\frac{e_{f^{\prime}} c_{f} a_{f}+e_{f} c_{f^{\prime}} a_{f^{\prime}}}{2}\left(\check{W}^{\mathrm{A} f f^{\prime}}\right)_{\mu \nu}^{\mathrm{ex}}(x)-\frac{e_{f^{\prime}} c_{f}}{2}\left(\check{W}_{5 \mathrm{a}}^{\mathrm{A} f f^{\prime}}\right)_{\mu \nu}^{\mathrm{ex}}(x)-\frac{e_{f} c_{f^{\prime}}}{2}\left(\check{W}_{5 \mathrm{~b}}^{\mathrm{A} f f^{\prime}}\right)_{\mu \nu}^{\mathrm{ex}}(x)\right]
\end{gathered}
$$

where we used eq. (5.74). In the momentum space this gives

$$
\begin{aligned}
W_{\mu \nu}^{\mathrm{I} 1 \mathrm{Aex}}(q)= & \frac{i N_{c}}{2\left(N_{c}^{2}-1\right) Q_{\|}^{2}} \sum_{f, f^{\prime}} \int d^{2} k_{\perp}\left\{c_{f} e_{f^{\prime}}\left[\epsilon_{\mu m} k^{m}(q-k)_{\nu}-\mu \leftrightarrow \nu\right] J_{+-}^{2 f f^{\prime}}\right. \\
& \left.-c_{f^{\prime}} e_{f}\left[k_{\mu} \epsilon_{\nu n}(q-k)^{n}-\mu \leftrightarrow \nu\right] J_{-+}^{2 f f^{\prime}}\right\}
\end{aligned}
$$

where we used eqs. (5.78) and (5.82). 


\subsubsection{The result for antisymmetric part of interference hadronic tensor $W^{\mathrm{I} 1}$}

As usual, the result consists of "annihilation" and "exchange" parts

$$
\begin{aligned}
& W_{\mu \nu}^{\mathrm{I} 1 \mathrm{~A}}(q)=W_{\mu \nu}^{\mathrm{I} 1 \mathrm{Aan}}(q)+W_{\mu \nu}^{\mathrm{I} 1 \mathrm{Aex}}(q), \\
& W_{\mu \nu}^{\mathrm{I} 1 \mathrm{Aan}}(q)=-i \epsilon_{\mu \nu \alpha \beta} q^{\alpha} \int d^{2} k_{\perp}\left(\tilde{q}+q_{\perp}-2 k_{\perp}\right)^{\beta} \sum_{f} e_{f} c_{f} \mathcal{F}^{f}\left(q, k_{\perp}\right) \text {, } \\
& W_{\mu \nu}^{\mathrm{I1} A \mathrm{Aex}}(q)=\text { r.h.s. of eq. }(6.11)
\end{aligned}
$$

where $\mathcal{F}^{f}\left(q, k_{\perp}\right)$ is given by eq. (5.27). Obviously, $q^{\mu} W_{\mu \nu}^{\mathrm{I1} \operatorname{Aan}}(q)=0$.

\subsection{Symmetric part of interference hadronic tensor $W^{\mathrm{I} 2}$}

From definitions (2.6) and (2.9) we get

$$
\begin{aligned}
\check{W}_{\mu \nu}^{\mathrm{I} 2 \mathrm{~S}}(x)= & \frac{N_{c}}{2 s} \sum_{f, f^{\prime}}\left(\left(e_{f^{\prime}} c_{f} a_{f}-e_{f} c_{f^{\prime}} a_{f^{\prime}}\right)\langle A, B|\left[\bar{\psi}(x) \gamma_{\mu} \psi(x)\right]^{f}\left[\bar{\psi}(0) \gamma_{\nu} \psi(0)\right]^{f^{\prime}}\right. \\
& +e_{f} c_{f^{\prime}}\langle A, B|\left[\bar{\psi}(x) \gamma_{\mu} \psi(x)\right]^{f}\left[\bar{\psi}(0) \gamma_{\nu} \gamma_{5} \psi(0)\right]^{f^{\prime}} \\
& \left.-e_{f^{\prime}} c_{f}\left\langle A, B\left|\left[\bar{\psi}(x) \gamma_{\mu} \gamma_{5} \psi(x)\right]^{f}\left[\bar{\psi}(0) \gamma_{\nu} \psi(0)\right]^{f^{\prime}}\right| A, B\right\rangle\right)+\mu \leftrightarrow \nu
\end{aligned}
$$

where $\psi=\Psi_{1}+\Psi_{2}$ in our approximation.

\subsubsection{Annihilation-type power corrections}

Let us start with annihilation-type power corrections. Since in this case $f=f^{\prime}$, the first term in the r.h.s. of eq. (6.13) vanishes and the second turns to

$$
\begin{aligned}
\check{W}_{\mu \nu}^{\mathrm{I} 2 \mathrm{San}}(x)= & \sum_{f} e_{f} c_{f} \check{W}_{\mu \nu}^{\mathrm{If}}(x) \\
\check{W}_{\mu \nu}^{\mathrm{If}}(x)= & \frac{N_{c}}{2 s}\langle A, B|\left[\bar{\Psi}_{1}(x) \gamma_{\mu} \bar{\Psi}_{2}(x)\right]\left[\bar{\Psi}_{2}(0) \gamma_{\nu} \gamma_{5} \bar{\Psi}_{1}(0)\right] \\
& -\left[\bar{\Psi}_{1}(x) \gamma_{\mu} \gamma_{5} \bar{\Psi}_{2}(x)\right]\left[\bar{\Psi}_{2}(0) \gamma_{\nu} \bar{\Psi}_{1}(0)\right]+\mu \leftrightarrow \nu|A, B\rangle-x \leftrightarrow 0
\end{aligned}
$$

After Fierz transformation (A.4) it turns to

$$
\begin{aligned}
\check{W}_{\mu \nu}^{\mathrm{I}}(x)= & -\frac{N_{c}}{4 s}\left\langle p_{A}, p_{B}\right|\left[\bar{\Psi}_{1}^{m}(x) \sigma_{\mu \xi} \Psi_{1}^{n}(0)\right]\left[\bar{\Psi}_{2}^{n}(0) \sigma_{\nu}^{\xi} \gamma_{5} \Psi_{2}^{m}(x)\right] \\
& \left.-\bar{\Psi}_{1}^{m}(x) \sigma_{\mu \xi} \gamma_{5} \Psi_{1}^{n}(0)\right]\left[\bar{\Psi}_{2}^{n}(0) \sigma_{\nu}{ }^{\xi} \Psi_{2}^{m}(x)\right]+\mu \leftrightarrow \nu\left|p_{A}, p_{B}\right\rangle \\
& +\frac{N_{c} g_{\mu \nu}}{2 s}\left\langle p_{A}, p_{B}\right|\left[\bar{\Psi}_{1}^{m}(x) \Psi_{1}^{n}(0)\right]\left[\bar{\Psi}_{2}^{n}(0) \gamma_{5} \Psi_{2}^{m}(x)\right] \\
& -\left[\bar{\Psi}_{1}^{m}(x) \gamma_{5} \Psi_{1}^{n}(0)\right]\left[\bar{\Psi}_{2}^{n}(0) \Psi_{1}^{m}(x)\right]\left|p_{A}, p_{B}\right\rangle-x \leftrightarrow 0
\end{aligned}
$$

where we suppressed flavor label. 
Let us start from the second term. Obviously, the leading-twist contribution vanishes with our accuracy. Next, consider one-gluon terms and start from

$$
\begin{aligned}
& \frac{N_{c} g_{\mu \nu}}{2 s}\left\langle p_{A}, p_{B}\right|\left(\left[\bar{\psi}_{A}^{m}(x) \Xi_{1}^{n}(0)\right]+\left[\bar{\Xi}_{1}^{m}(x) \Psi_{1}^{n}(0)\right)\left[\bar{\psi}_{B}^{n}(0) \gamma_{5} \psi_{B}^{m}(x)\right]\right. \\
& -\left(\left[\bar{\psi}_{A}^{m}(x) \gamma_{5} \Xi_{1}^{n}(0)\right]+\left[\bar{\Xi}_{1}^{m}(x) \gamma_{5} \Psi_{1}^{n}(0)\right)\left[\bar{\psi}_{B}^{n}(0) \psi_{B}^{m}(x)\right]\left|p_{A}, p_{B}\right\rangle-x \leftrightarrow 0\right. \\
& =\frac{i g_{\mu \nu}}{2 s^{2}}\left\{\left\langle\bar{\psi}(x) \sigma_{\star i} \frac{1}{\alpha} \psi(0)\right\rangle_{A}\left\langle\bar{\psi} B^{i}(0) \gamma_{5} \psi(x)\right\rangle_{B}-\left\langle\left(\bar{\psi} \frac{1}{\alpha}\right)(x) \sigma_{\star i} \psi(0)\right\rangle_{A}\left\langle\bar{\psi}(0) \gamma_{5} B^{i} \psi(x)\right\rangle_{B}\right. \\
& \left.-\left\langle\bar{\psi}(x) \sigma_{\star i} \gamma_{5} \frac{1}{\alpha} \psi(0)\right\rangle_{A}\left\langle\bar{\psi} B^{i}(0) \psi(x)\right\rangle_{B}+\left\langle\left(\bar{\psi} \frac{1}{\alpha}\right)(x) \sigma_{\star i} \gamma_{5} \psi(0)\right\rangle_{A}\left\langle\bar{\psi}(0) B^{i} \psi(x)\right\rangle_{B}\right\}-x \leftrightarrow 0
\end{aligned}
$$

The projectile matrix element can bring one factor of $s$ while the target one cannot so the contribution to $\check{W}_{\mu \nu}^{\mathrm{I}}(x)$ is $\sim \frac{g_{\mu \nu}}{\alpha_{q} s}$ which is $O\left(\beta_{q}\right) \times \frac{q_{\mu \nu}}{Q_{\|}^{2}}$. Similarly, the contributions coming from $\Xi_{2}$ and $\bar{\Xi}_{1}$ are $\sim \frac{g_{\mu \nu}}{\beta_{q} s}$ and can be neglected.

Let us consider now two-gluon term coming from $\Xi_{1}$ and $\Xi_{2}$

$$
\begin{aligned}
\frac{N_{c} g_{\mu \nu}}{2 s}\left\langle p_{A}, p_{B}\right|\left[\bar{\psi}_{A}^{m}(x) \Xi_{1}^{n}(0)\right]\left[\bar{\psi}_{B}^{n}(0) \gamma_{5} \Xi_{2}^{m}(x)\right] \\
-\left[\bar{\psi}_{A}^{m}(x) \gamma_{5} \Xi_{1}^{n}(0)\right]\left[\bar{\psi}_{B}^{n}(0) \Xi_{2}^{m}(x)\right]\left|p_{A}, p_{B}\right\rangle-x \leftrightarrow 0 \\
=\frac{g_{\mu \nu}}{2 s^{3}}\left\{\left\langle\bar{\psi} A^{j}(x) \sigma_{\star i} \frac{1}{\alpha} \psi(0)\right\rangle_{A}\left\langle\bar{\psi} B^{i}(0) \sigma_{\bullet j} \gamma_{5} \frac{1}{\beta} \psi(x)\right\rangle_{B}\right. \\
\left.\quad-\left\langle\bar{\psi} A^{j}(x) \sigma_{\star i} \frac{1}{\alpha} \psi(0)\right\rangle_{A}\left\langle\bar{\psi} B^{i}(0) \sigma_{\bullet j} \gamma_{5} \frac{1}{\beta} \psi(x)\right\rangle_{B}\right\}-x \leftrightarrow 0 \\
=\frac{g_{\mu \nu}}{2 s^{3}}\left\{\left\langle\bar{\psi}(x)\left(A^{j}(x) \sigma_{\star i}-A^{i}(x) \sigma_{\bullet j}\right) \frac{1}{\alpha} \psi(0)\right\rangle_{A}\left\langle\bar{\psi} B^{i}(0) \sigma_{\bullet j} \gamma_{5} \frac{1}{\beta} \psi(x)\right\rangle_{B}\right. \\
\left.\quad+\left\langle\bar{\psi} A^{i}(x) \sigma_{\star i} \frac{1}{\alpha} \psi(0)\right\rangle_{A}\left\langle\bar{\psi} B^{j}(0) \sigma_{\bullet j} \gamma_{5} \frac{1}{\beta} \psi(x)\right\rangle_{B}\right\}-x \leftrightarrow 0 \\
=-\frac{g_{\mu \nu}}{2 s^{3}}\left\langle\bar{\psi}(x) \not A(x) \not p_{2} \frac{1}{\alpha} \psi(0)\right\rangle_{A}\left\langle\bar{\psi}(0) \not B(0) \not p_{1} \gamma_{5} \frac{1}{\beta} \psi(x)\right\rangle_{B}-x \leftrightarrow 0
\end{aligned}
$$

where again we used eq. (A.13) without two last terms and eq. (4.34). Now, from equations of motion (A.54) and (A.55) we see that the target matrix element vanishes:

$$
\begin{aligned}
& \int d x e^{-i \beta_{q} x_{\star}+i(k, x)_{\perp}}\left\langle\bar{\psi}(0) \not B(0) \not p_{1} \gamma_{5} \frac{1}{\beta} \psi(x)\right\rangle_{B} \\
& =\frac{k_{i}^{\perp}}{\beta_{q}} \int d x e^{-i \beta x_{\star}+i(k, x)_{\perp}}\left\langle\bar{\psi}(0) \gamma^{i} \not p_{1} \gamma_{5} \psi(x)\right\rangle_{B} \sim \frac{k_{i}^{\perp}}{\beta_{q}} s \epsilon^{i j} k_{j}=0
\end{aligned}
$$

It is easy to see that the term coming from $\bar{\Xi}_{1}$ and $\bar{\Xi}_{2}$ vanishes for the same reason.

$$
\begin{aligned}
& \frac{N_{c} g_{\mu \nu}}{2 s}\left\langle p_{A}, p_{B}\right|\left[\bar{\Xi}_{1}^{m}(x) \Psi_{1}^{n}(0)\right]\left[\bar{\Xi}_{2}^{n}(0) \gamma_{5} \psi_{B}^{m}(x)\right] \\
& -\left[\bar{\Xi}_{1}^{m}(x) \gamma_{5} \Psi_{1}^{n}(0)\right]\left[\bar{\Xi}_{2}^{n}(0) \psi_{B}^{m}(x)\right]\left|p_{A}, p_{B}\right\rangle-x \leftrightarrow 0 \\
& \quad=-\frac{g_{\mu \nu}}{2 s^{3}}\left\langle\left(\bar{\psi} \frac{1}{\alpha}\right)(x) \not p_{2} A(0) \psi(0)\right\rangle_{A}\left\langle\left(\bar{\psi} \frac{1}{\beta}\right)(0) \not p_{1} \not B(x) \gamma_{5} \psi(x)\right\rangle_{B}-x \leftrightarrow 0=0
\end{aligned}
$$


Next, two-gluon contribution from $\bar{\Xi}_{1}$ and $\Xi_{1}$ vanishes since $\bar{\Xi}_{1} \Xi_{1}=0$, and similarly $\bar{\Xi}_{2} \Xi_{2}=0$. Finally, the contribution coming from $\bar{\Xi}_{1}$ and $\Xi_{2}$ is $\sim \frac{1}{N_{c}^{2}}$ as demonstrated in section 4.3 .2 (see eq. (4.63)), so the second term in eq. (6.15) vanishes and we get

$$
\begin{aligned}
\check{W}_{\mu \nu}^{\mathrm{I}}(x)= & -\frac{N_{c}}{4 s}\left\langle p_{A}, p_{B}\right|\left[\bar{\Psi}_{1}^{m}(x) \sigma_{\mu \xi} \Psi_{1}^{n}(0)\right]\left[\bar{\Psi}_{2}^{n}(0) \sigma_{\nu}{ }^{\xi} \gamma_{5} \Psi_{2}^{m}(x)\right] \\
& \left.\left.-\bar{\Psi}_{1}^{m}(x) \sigma_{\mu \xi} \gamma_{5} \Psi_{1}^{n}\right)(0)\right]\left[\bar{\Psi}_{2}^{n}(0) \sigma_{\nu}{ }^{\xi} \Psi_{2}^{m}(x)\right]+\mu \leftrightarrow \nu\left|p_{A}, p_{B}\right\rangle-x \leftrightarrow 0
\end{aligned}
$$

This contribution is similar to eq. (4.39) up to extra $\gamma_{5}$ and relative signs. As we discussed above, extra $\gamma_{5}$ cannot change the power of our small parameters $\frac{q_{\perp}^{2}}{Q^{2}}$ and $\alpha_{q}, \beta_{q}$ so we can consider only terms which gave leading contributions to $V_{\mu \nu}^{\mathrm{H}}$.

The leading-twist contribution

$$
\begin{aligned}
\check{W}_{\mu \nu}^{\text {I,l.t. }}(x)= & -\frac{1}{4 s}\left\langle p_{A}, p_{B}\right|\left[\bar{\psi}_{A}(x) \sigma_{\mu \xi} \psi_{A}(0)\right]\left[\bar{\psi}_{B}(0) \sigma_{\nu}{ }^{\xi} \gamma_{5} \psi_{B}(x)\right] \\
& -\left[\bar{\psi}_{A}(x) \sigma_{\mu \xi} \gamma_{5} \psi_{A}(0)\right]\left[\bar{\psi}_{B}(0) \sigma_{\nu}^{\xi} \psi_{B}(x)\right]+\mu \leftrightarrow \nu\left|p_{A}, p_{B}\right\rangle-x \leftrightarrow 0
\end{aligned}
$$

is easily obtained from parametrizations (A.44)

$$
\begin{aligned}
W_{\mu \nu}^{\mathrm{I}, \mathrm{lt}}(x)(q) & =\frac{1}{16 \pi^{4}} \int d x_{\bullet} d x_{\star} d^{2} x_{\perp} e^{-i \alpha_{q} x_{\bullet}-i \beta_{q} x_{\star}+i(q, x)_{\perp}} \check{W}_{\mu \nu}^{\mathrm{I}, \mathrm{lt}}(x) \\
& =-\frac{i}{2 m^{2}} \int d^{2} k_{\perp}\left(\epsilon_{\mu j}\left[k^{j}(q-k)_{\nu}^{\perp}+(q-k)_{j} k_{\nu}^{\perp}\right]+\mu \leftrightarrow \nu\right) \mathcal{H}^{f}\left(q, k_{\perp}\right)
\end{aligned}
$$

where

$$
\mathcal{H}^{f}\left(q, k_{\perp}\right)=h_{1}^{\perp}\left(\alpha_{q}, k_{\perp}\right) \bar{h}_{1}^{\perp}\left(\beta_{q},(q-k)_{\perp}\right)-h_{1 f}^{\perp} \leftrightarrow \bar{h}_{1 f}^{\perp}
$$

As usually, the term with $h_{1 f}^{\perp} \leftrightarrow \bar{h}_{1 f}^{\perp}$ comes from $x \leftrightarrow 0$ contribution.

Next, we need to consider terms in eq. (6.20) with one or two gluon operators and generalize the calculations from section 4.3 to our case.

\subsubsection{One-gluon terms}

Let us first consider term coming from $\Xi_{1}(0)=\frac{i}{s} \sigma_{\star i} B^{i} \frac{1}{\alpha} \psi_{A}$. Separating color-singlet matrix elements in eq. (6.15), we get

$$
\begin{aligned}
\check{W}_{1 \mu \nu}^{1 \mathrm{I}(1)}(x)= & -\frac{i}{4 s^{2}}\left\{\left\langle\bar{\psi}(x) \sigma_{\mu \xi} \sigma_{\star i} \frac{1}{\alpha} \psi(0)\right\rangle_{A}\left\langle\bar{\psi} B^{i}(0) \sigma_{\nu}{ }^{\xi} \gamma_{5} \psi(x)\right]\right\rangle_{B} \\
& \left.-\psi(0) \otimes \gamma_{5} \psi(x) \leftrightarrow \gamma_{5} \psi(0) \otimes \psi(x)+\mu \leftrightarrow \nu\right\}-x \leftrightarrow 0
\end{aligned}
$$

As we mentioned, this contribution is similar to the one considered in section 4.3.1 so we need to take only the case of longitudinal $\mu$ and transverse $\nu$, or vice versa - all other 
cases are power-suppressed. We get

$$
\begin{aligned}
\check{W}_{1 \mu \nu}^{1 \mathrm{II}(1)}(x)= & -\frac{i p_{2 \mu}}{2 s^{3}}\left\{\left\langle\bar{\psi}(x) \sigma_{\bullet \xi} \sigma_{\star i} \frac{1}{\alpha} \psi(0)\right\rangle_{A}\left\langle\bar{\psi} B^{i}(0) \sigma_{\nu_{\perp}}^{\xi} \gamma_{5} \psi(x)\right]\right\rangle_{B} \\
& \left.+\left\langle\bar{\psi}(x) \sigma_{\nu_{\perp} \xi} \sigma_{\star i} \frac{1}{\alpha} \psi(0)\right\rangle_{A}\left\langle\bar{\psi} B^{i}(0) \sigma_{\bullet} \gamma_{5} \psi(x)\right]\right\rangle_{B} \\
& \left.-\psi(0) \otimes \gamma_{5} \psi(x) \leftrightarrow \gamma_{5} \psi(0) \otimes \psi(x)\right\}-x \leftrightarrow 0 \\
= & -\frac{i p_{2 \mu}}{2 s^{3}}\left\{\left\langle\bar{\psi}(x) \sigma_{\nu_{\perp} j} \sigma_{\star i} \frac{1}{\alpha} \psi(0)\right\rangle_{A}\left\langle\bar{\psi} B^{i}(0) \sigma_{\bullet}{ }^{j} \gamma_{5} \psi(x)\right]\right\rangle_{B} \\
& \left.-i\left\langle\bar{\psi}(x) \sigma_{\star i} \frac{1}{\alpha} \psi(0)\right\rangle_{A}\left\langle\bar{\psi} B^{i}(0) \sigma_{\nu_{\perp} \bullet} \gamma_{5} \psi(x)\right]\right\rangle_{B} \\
& \left.+\left\langle\bar{\psi}(x) \sigma_{\bullet j} \sigma_{\star i} \frac{1}{\alpha} \psi(0)\right\rangle_{A}\left\langle\bar{\psi} B^{i}(0) \sigma_{\nu_{\perp}}^{j} \gamma_{5} \psi(x)\right]\right\rangle_{B} \\
& \left.+\frac{2}{s}\left\langle\bar{\psi}(x) \sigma_{\nu_{\perp}} \bullet \sigma_{\star i} \frac{1}{\alpha} \psi(0)\right\rangle_{A}\left\langle\bar{\psi} B^{i}(0) \sigma_{\bullet \star} \gamma_{5} \psi(x)\right]\right\rangle_{B} \\
& \left.-\psi(0) \otimes \gamma_{5} \psi(x) \leftrightarrow \gamma_{5} \psi(0) \otimes \psi(x)\right\}-x \leftrightarrow 0
\end{aligned}
$$

where we used power-counting results from ref. [16] to eliminate terms proportional to $p_{1 \mu}$, cf eq. (4.42). Moreover, similarly to eq. (4.42) case, eq. (A.9) shows that two last terms in the r.h.s. of eq. (6.25) are small and therefore

$$
\begin{aligned}
\check{W}_{1 \mu \nu}^{\mathrm{II}(1)}(x)= & -\frac{p_{2 \mu}}{2 s^{3}}\left\{\left\langle\bar{\psi}(x)\left[g_{i \nu_{\perp}} \sigma_{\star j}-g_{i j} \sigma_{\star \nu_{\perp}}\right] \frac{1}{\alpha} \psi(0)\right\rangle_{A}\left\langle\bar{\psi} B^{i}(0) \sigma_{\bullet}^{j} \gamma_{5} \psi(x)\right\rangle_{B}\right. \\
& -\left[\left\langle\bar{\psi}(x) \sigma_{\star i} \frac{1}{\alpha} \psi(0)\right\rangle_{A}\left\langle\bar{\psi} B^{i}(0) \sigma_{\bullet \nu_{\perp}} \gamma_{5} \psi(x)\right]\right\rangle_{B} \\
& \left.\left.-\psi(0) \otimes \gamma_{5} \psi(x) \leftrightarrow \gamma_{5} \psi(0) \otimes \psi(x)\right]\right\}-x \leftrightarrow 0 \\
= & -\frac{p_{2 \mu}}{2 s^{3}}\left\{\left\langle\bar{\psi}(x) \sigma_{\star j} \frac{1}{\alpha} \psi(0)\right\rangle_{A}\left\langle\bar{\psi}(0)\left[B_{\nu}(0) \sigma_{\bullet}^{j}-B^{j}(0) \sigma_{\bullet \nu_{\perp}}\right] \gamma_{5} \psi(x)\right\rangle_{B}\right. \\
& \left.+\left\langle\bar{\psi}(x) \sigma_{\star \nu_{\perp}} \gamma_{5} \frac{1}{\alpha} \psi(0)\right\rangle_{A}\left\langle\bar{\psi} B^{i}(0) \sigma_{\bullet i} \psi(x)\right\rangle_{B}\right\}-x \leftrightarrow 0
\end{aligned}
$$

where we used eqs. (A.9), (4.34), and (6.18). Next, the target matrix element in the first term in the r.h.s. can be rewritten as

$$
\left.\left\langle\bar{\psi}(0)\left[B_{\nu}(0) \sigma_{\bullet}^{j}-B^{j}(0) \sigma_{\bullet \nu_{\perp}}\right] \gamma_{5} \psi(x)\right]\right\rangle_{B}=\epsilon_{\nu j}\left\langle\bar{\psi}(0) \not B(0) \not p_{1} \psi(x)\right\rangle_{B}
$$

so we get

$$
\check{W}_{1 \mu \nu}^{1 \mathrm{I}(1)}(x)=-\frac{p_{2 \mu}}{s^{3}} \epsilon_{\nu j}\left\langle\bar{\psi}(x) \sigma_{\star j} \frac{1}{\alpha} \psi(0)\right\rangle_{A}\left\langle\bar{\psi}(0) \not B(0) \not p_{1} \psi(x)\right\rangle_{B}-x \leftrightarrow 0
$$

where we used $\sigma_{\star \nu_{\perp}} \gamma_{5}=i \epsilon_{\nu j} \sigma_{\star}^{j}$. Now, from equation of motion (A.56) and parametrization (A.44) we get the corresponding contribution to $\check{W}_{\mu \nu}^{1 \mathrm{I}}(x)$ in the form

$$
\check{W}_{1 \mu \nu}^{1 \mathrm{II}(1)}(x)=-i \epsilon_{\nu j} \frac{p_{2 \mu}}{\alpha_{q} s m^{2}} \int d^{2} k_{\perp} k_{\perp}^{j}(q-k)_{\perp}^{2} \mathcal{H}\left(q, k_{\perp}\right)
$$


The term in eq. (6.20) coming from $\bar{\Xi}_{1}$ is similar:

$$
\begin{aligned}
\check{W}_{2 \mu \nu}^{\mathrm{II}(1)}(x)= & \frac{i}{4 s^{2}}\left\{\left\langle\left(\bar{\psi} \frac{1}{\alpha}\right)(x) \sigma_{\mu \xi} \sigma_{\star i} \psi(0)\right\rangle_{A}\left\langle\bar{\psi}(0) \sigma_{\nu}{ }^{\xi} \gamma_{5} B^{i}(x) \psi(x)\right]\right\rangle_{B} \\
& \left.-\psi(0) \otimes \gamma_{5} \psi(x) \leftrightarrow \gamma_{5} \psi(0) \otimes \psi(x)+\mu \leftrightarrow \nu\right\}-x \leftrightarrow 0 \\
= & \frac{i p_{2 \mu}}{2 s^{3}}\left\{\left\langle\left(\bar{\psi} \frac{1}{\alpha}\right)(x) \sigma_{\star i} \sigma_{\bullet \xi} \psi(0)\right\rangle_{A}\left\langle\bar{\psi} B^{i}(x) \sigma_{\nu_{\perp}}^{\xi} \gamma_{5} \psi(x)\right]\right\rangle_{B} \\
& \left.+\left\langle\left(\bar{\psi} \frac{1}{\alpha}\right)(x) \sigma_{\star i} \sigma_{\nu_{\perp} \xi} \frac{1}{\alpha} \psi(0)\right\rangle_{A}\left\langle\bar{\psi} B^{i}(x) \sigma_{\bullet}^{\xi} \gamma_{5} \psi(x)\right]\right\rangle_{B} \\
& \left.-\psi(0) \otimes \gamma_{5} \psi(x) \leftrightarrow \gamma_{5} \psi(0) \otimes \psi(x)\right\}-x \leftrightarrow 0 \\
= & \frac{i p_{2 \mu}}{2 s^{3}}\left\{\left\langle\left(\bar{\psi} \frac{1}{\alpha}\right)(x) \sigma_{\star i} \sigma_{\nu_{\perp} j} \psi(0)\right\rangle_{A}\left\langle\bar{\psi} B^{i}(x) \sigma_{\bullet}^{j} \gamma_{5} \psi(x)\right]\right\rangle_{B} \\
& \left.-i\left\langle\left(\bar{\psi} \frac{1}{\alpha}\right)(x) \sigma_{\star i} \frac{1}{\alpha} \psi(0)\right\rangle_{A}\left\langle\bar{\psi} B^{i}(x) \sigma_{\bullet \nu_{\perp}} \gamma_{5} \psi(x)\right]\right\rangle_{B} \\
& \left.-\psi(0) \otimes \gamma \gamma_{5} \psi(x) \leftrightarrow \gamma_{5} \psi(0) \otimes \psi(x)\right\}-x \leftrightarrow 0 \\
= & -\frac{p_{2 \mu}}{2 s^{3}}\left\{\left\langle\left(\bar{\psi} \frac{1}{\alpha}\right)(x)\left[g_{i \nu_{\perp}} \sigma_{\star j}-g_{i j} \sigma_{\star \nu_{\perp}}\right] \psi(0)\right\rangle_{A}\left\langle\bar{\psi} B^{i}(x) \sigma_{\bullet}^{j} \gamma_{5} \psi(x)\right\rangle_{B}\right. \\
& -\left[\left\langle\left(\bar{\psi} \frac{1}{\alpha}\right)(x) \sigma_{\star i} \psi(0)\right\rangle_{A}\left\langle\bar{\psi} B^{i}(x) \sigma_{\bullet \nu_{\perp}} \gamma_{5} \psi(x)\right]\right\rangle_{B} \\
& \left.\left.-\psi(0) \otimes \gamma_{5} \psi(x) \leftrightarrow \gamma_{5} \psi(0) \otimes \psi(x)\right]\right\}-x \leftrightarrow 0 \\
= & -\frac{p_{2 \mu}}{2 s^{3}}\left\{\left\langle\left(\bar{\psi} \frac{1}{\alpha}\right)(x) \sigma_{\star j} \psi(0)\right\rangle_{A}\left\langle\bar{\psi}(0)\left[B_{\nu}(x) \sigma_{\bullet}^{j}-B^{j}(x) \sigma_{\bullet \nu_{\perp}}\right] \gamma_{5} \psi(x)\right\rangle_{B}\right. \\
& \left.+\left\langle\left(\bar{\psi} \frac{1}{\alpha}\right)(x) \sigma_{\star \nu_{\perp}} \gamma_{5} \psi(0)\right\rangle_{A}\left\langle\bar{\psi} B^{i}(x) \sigma_{\bullet i} \psi(x)\right\rangle_{B}\right\}-x \leftrightarrow 0 \\
= & \frac{p_{2 \mu}}{s^{3}} \epsilon_{\nu j}\left\langle\left(\bar{\psi} \frac{1}{\alpha}\right)(x) \sigma_{\star j} \psi(0)\right\rangle_{A}\left\langle\bar{\psi}(0) \not b_{1} \not B(x) \psi(x)\right\rangle_{B}-x \leftrightarrow 0 \\
&
\end{aligned}
$$

where we left only the terms similar to the leading terms in eq. (6.25) and made the same transformations.

Now, from equation of motion (A.56) and parametrization (A.44) we get the corresponding contribution to $\check{W}_{\mu \nu}^{1 \mathrm{I}}(x)$ in the form

$$
\check{W}_{2 \mu \nu}^{1 \mathrm{I}(1)}(x)=-i \epsilon_{\nu j} \frac{p_{2 \mu}}{\alpha_{q} s m^{2}} \int d^{2} k_{\perp} k_{\perp}^{j}(q-k)_{\perp}^{2} \mathcal{H}\left(q, k_{\perp}\right)
$$

so it doubles the contribution (6.29). 
Let us now consider term in eq. (6.20) coming form $\Xi_{2}$. For longitudinal $\mu$ and transverse $\nu$ we get

$$
\begin{aligned}
\check{W}_{1 \mu \nu}^{1 \mathrm{II}(2)}(x)= & -\frac{i}{4 s^{2}}\left\{\left\langle\bar{\psi} A^{i}(x) \sigma_{\mu \xi} \psi(0)\right\rangle_{A}\left\langle\bar{\psi}(0) \sigma_{\nu}^{\xi} \sigma_{\bullet i} \gamma_{5} \frac{1}{\beta} \psi(x)\right\rangle_{B}\right. \\
& \left.-\psi(0) \otimes \gamma_{5} \psi(x) \leftrightarrow \gamma_{5} \psi(0) \otimes \psi(x)+\mu \leftrightarrow \nu\right\}-x \leftrightarrow 0 \\
= & -\frac{i p_{1 \mu}}{2 s^{3}}\left\{\left\langle\bar{\psi} A^{i}(x) \sigma_{\star \xi} \psi(0)\right\rangle_{A}\left\langle\bar{\psi}(0) \sigma_{\nu_{\perp}}^{\xi} \sigma_{\bullet i} \gamma_{5} \frac{1}{\beta} \psi(x)\right\rangle_{B}\right. \\
& +\left\langle\bar{\psi} A^{i}(x) \sigma_{\nu_{\perp} \xi} \psi(0)\right\rangle_{A}\left\langle\bar{\psi}(0) \sigma_{\star}^{\xi} \sigma_{\bullet} \gamma_{5} \frac{1}{\beta} \psi(x)\right\rangle_{B} \\
& \left.-\psi(0) \otimes \gamma_{5} \psi(x) \leftrightarrow \gamma_{5} \psi(0) \otimes \psi(x)\right\}-x \leftrightarrow 0 \\
= & -\frac{i p_{1 \mu}}{2 s^{3}}\left\{\left\langle\bar{\psi} A^{i}(x) \sigma_{\star j} \psi(0)\right\rangle_{A}\left\langle\bar{\psi}(0) \sigma_{\nu_{\perp}}^{j} \sigma_{\bullet i} \gamma_{5} \frac{1}{\beta} \psi(x)\right\rangle_{B}\right. \\
& -i\left\langle\bar{\psi} A^{i}(x) \sigma_{\nu_{\perp} \star} \psi(0)\right\rangle_{A}\left\langle\bar{\psi}(0) \sigma_{\bullet i} \gamma_{5} \frac{1}{\beta} \psi(x)\right\rangle_{B} \\
& +\left\langle\bar{\psi} A^{i}(x) \sigma_{\nu_{\perp} j} \psi(0)\right\rangle_{A}\left\langle\bar{\psi}(0) \sigma_{\star}^{j} \sigma_{\bullet i} \gamma_{5} \frac{1}{\beta} \psi(x)\right\rangle_{B}+\frac{2}{s}\left\langle\bar{\psi} A^{i}(x) \sigma_{\star \bullet} \psi(0)\right\rangle_{A} \\
& \left.\times\left\langle\bar{\psi}(0) \sigma_{\nu_{\perp} \star} \sigma_{\bullet i} \gamma_{5} \frac{1}{\beta} \psi(x)\right\rangle_{B}-\psi(0) \otimes \gamma_{5} \psi(x) \leftrightarrow \gamma_{5} \psi(0) \otimes \psi(x)\right\}-x \leftrightarrow 0
\end{aligned}
$$

where we neglected contribution $\sim p_{2 \mu}$ since it is $\sim p_{2 \mu} q_{\nu}^{\perp} \frac{m_{\perp}^{2}}{\beta_{q} s^{2}}$, see power counting (without $\gamma_{5}$ ) in ref. [16]. Next, similarly to eq. (6.25), the last two terms in the r.h.s. of eq. (6.32) are $\sim O\left(\frac{m_{\perp}^{2}}{s}\right)$ in comparison to the first two terms so we get

$$
\begin{aligned}
\check{W}_{1 \mu \nu}^{1 \mathrm{I}(2)}(x)= & -\frac{p_{1 \mu}}{2 s^{3}}\left\{\left\langle\bar{\psi} A^{i}(x) \sigma_{\star j} \psi(0)\right\rangle_{A}\left\langle\bar{\psi}(0)\left[g_{i \nu_{\perp}} \sigma_{\bullet j}-g_{i j} \sigma_{\bullet \nu_{\perp}}\right] \gamma_{5} \frac{1}{\beta} \psi(x)\right\rangle_{B}\right. \\
& \left.-\left\langle\bar{\psi} A^{i}(x) \sigma_{\star \nu_{\perp}} \psi(0)\right\rangle_{A}\left\langle\bar{\psi}(0) \sigma_{\bullet i} \gamma_{5} \frac{1}{\beta} \psi(x)\right\rangle_{B}-\psi(0) \otimes \gamma_{5} \psi(x) \leftrightarrow \gamma_{5} \psi(0) \otimes \psi(x)\right\} \\
& -x \leftrightarrow 0 \\
= & \frac{p_{1 \mu}}{s^{3}}\left\{\left\langle\bar{\psi}(x)\left(A_{\nu}(x) \sigma_{\star j}-A_{j}(x) \sigma_{\star \nu_{\perp}}\right) \gamma_{5} \psi(0)\right\rangle_{A}\left\langle\bar{\psi}(0) \sigma_{\bullet}{ }^{j} \frac{1}{\beta} \psi(x)\right\rangle_{B}\right. \\
& \left.+\left\langle\bar{\psi} A^{i}(x) \sigma_{\star i} \psi(0)\right\rangle_{A}\left\langle\bar{\psi}(0) \sigma_{\bullet \nu_{\perp}} \gamma_{5} \frac{1}{\beta} \psi(x)\right\rangle_{B}\right\}-x \leftrightarrow 0 \\
= & -\frac{i p_{1 \mu}}{s^{3}} \epsilon_{\nu_{\perp} j}\left\langle\bar{\psi} A^{i}(x) \sigma_{\star i} \psi(0)\right\rangle_{A}\left\langle\bar{\psi}(0) \sigma_{\bullet}{ }^{j} \frac{1}{\beta} \psi(x)\right\rangle_{B}
\end{aligned}
$$

From eq. (A.56) we get the corresponding contribution to $W_{\mu \nu}^{\mathrm{I}}(q)$ in the form

$$
\check{W}_{1 \mu \nu}^{1 \mathrm{I}(2)}(q)=-i \epsilon_{\nu j} \frac{p_{1 \mu}}{\beta_{q} s m^{2}} \int d^{2} k_{\perp}(q-k)_{\perp}^{j} k_{\perp}^{2} \mathcal{H}\left(q, k_{\perp}\right)
$$

Similarly to eq. (6.29) one can demonstrate that the term coming from $\bar{\Xi}_{2}$ doubles the 
result (6.34) of $\Xi_{2}$ so we finally get

$$
W_{\mu \nu}^{1 \mathrm{I}}(q)=-i \frac{2 \epsilon_{\nu j}}{Q_{\|}^{2} m^{2}} \int d^{2} k_{\perp}\left[\beta p_{2 \mu} k_{\perp}^{j}(q-k)_{\perp}^{2}+\alpha p_{1 \mu}(q-k)^{j} k_{\perp}^{2}\right] \mathcal{H}\left(q, k_{\perp}\right)+\mu \leftrightarrow \nu
$$

where we have added the contribution of transverse $\mu$ and longitudinal $\nu$.

\subsubsection{Two-gluon terms}

Let us start from the contribution to $\check{W}_{\mu \nu}^{\mathrm{I}}(x)$ of eq. (6.20) coming from $\Xi_{A}$ and $\Xi_{B}$. After separation of color-singlet matrix elements, it takes the form

$$
\begin{aligned}
\check{W}_{\mu \nu}^{2 \mathrm{II}(1)}(x)= & \frac{1}{4 s^{3}}\left\{\left\langle\bar{\psi} A^{i}(x) \sigma_{\mu \xi} \sigma_{\star j} \frac{1}{\alpha} \psi(0)\right\rangle_{A}\left\langle\bar{\psi} B^{j}(0) \sigma_{\nu}{ }_{\nu} \sigma_{\bullet i} \gamma_{5} \frac{1}{\beta} \psi(x)\right\rangle_{B}\right. \\
& \left.-\psi(0) \otimes \gamma_{5} \psi(x) \leftrightarrow \gamma_{5} \psi(0) \otimes \psi(x)+\mu \leftrightarrow \nu\right\}-x \leftrightarrow 0
\end{aligned}
$$

This equation resembles $\check{V}_{1 \mu \nu}^{(2 \mathrm{a}) \mathrm{H}}(x)$ of eq. (4.47) calculated in section 4.3.2 so we will use power counting from that section and consider only two transverse or two longitudinal indices.

First, let us consider transverse $\mu$ and $\nu$.

$$
\begin{aligned}
\check{W}_{\mu \nu}^{2 \mathrm{I}(1)}(x)= & \frac{1}{4 s^{3}}\left\{\left\langle\bar{\psi} A^{i}(x) \sigma_{\mu_{\perp} k} \sigma_{\star j} \frac{1}{\alpha} \psi(0)\right\rangle_{A}\left\langle\bar{\psi} B^{j}(0) \sigma_{\nu_{\perp}}^{k} \sigma_{\bullet i} \gamma_{5} \frac{1}{\beta} \psi(x)\right\rangle_{B}\right. \\
& +\frac{2}{s}\left\langle\bar{\psi} A^{i}(x) \sigma_{\mu_{\perp} \bullet \sigma_{\star j}} \psi(0)\right\rangle_{A}\left\langle\bar{\psi} B^{j}(0) \sigma_{\nu_{\perp} \star} \sigma_{\bullet i} \gamma_{5} \frac{1}{\beta} \psi(x)\right\rangle_{B} \\
& \left.-\psi(0) \otimes \gamma_{5} \psi(x) \leftrightarrow \gamma_{5} \psi(0) \otimes \psi(x)+\mu \leftrightarrow \nu\right\}-x \leftrightarrow 0
\end{aligned}
$$

Similarly to eq. (4.48), from eqs. (A.9) and (4.34), we see that the second term in the r.h.s. can be neglected in comparison to the first one and after some algebra we get

$$
\begin{aligned}
\check{W}_{\mu_{\perp} \nu_{\perp}}^{2 \mathrm{I}(1)}(x) \\
=\frac{-1}{4 s^{3}}\left\{\left\langle\bar{\psi} A^{i}(x)\left[g_{\mu j} \sigma_{\star k}-g_{j k} \sigma_{\star \mu_{\perp}}\right] \frac{1}{\alpha} \psi(0)\right\rangle_{A}\left\langle\bar{\psi} B^{j}(0)\left[g_{\nu i} \sigma_{\bullet}{ }^{k}-\delta_{i}^{k} \sigma_{\bullet \nu_{\perp}}\right] \gamma_{5} \frac{1}{\beta} \psi(x)\right\rangle_{B}\right. \\
\left.\quad-\psi(0) \otimes \gamma_{5} \psi(x) \leftrightarrow \gamma_{5} \psi(0) \otimes \psi(x)+\mu \leftrightarrow \nu\right\}-x \leftrightarrow 0 \\
=\frac{1}{2 s^{3}}\left[\left\langle\bar{\psi}(x)\left[A_{\nu}(x) \sigma_{\star i}-A_{i}(x) \sigma_{\star \nu}\right] \frac{1}{\alpha} \psi(0)\right\rangle_{A}\left\langle\bar{\psi}\left(B_{\mu}(0) \sigma_{\bullet}^{i}-B^{i}(0) \sigma_{\bullet \mu}\right) \gamma_{5} \frac{1}{\beta} \psi(x)\right\rangle_{B}\right. \\
\quad+\left\langle\bar{\psi} A_{\nu}(x) \sigma_{\star k} \frac{1}{\alpha} \psi(0)\right\rangle_{A}\left\langle\bar{\psi} B^{i}(0) \sigma_{\bullet j} \gamma_{5} \frac{1}{\beta} \psi(x)\right\rangle_{B}\left(\delta_{i}^{k} \delta_{\mu}^{j}-\delta_{i}^{j} \delta_{\mu}^{k}\right) \\
\quad+\left\langle\bar{\psi} A^{i}(x) \sigma_{\star j} \frac{1}{\alpha} \psi(0)\right\rangle_{A}\left\langle\bar{\psi} B_{\mu}(0) \sigma_{\bullet k} \gamma_{5} \frac{1}{\beta} \psi(x)\right\rangle_{B}\left(\delta_{i}^{k} \delta_{\nu}^{j}-\delta_{\nu}^{k} \delta_{i}^{j}\right) \\
\left.\quad-\psi(0) \otimes \gamma_{5} \psi(x) \leftrightarrow \gamma_{5} \psi(0) \otimes \psi(x)\right]+\mu \leftrightarrow \nu-x \leftrightarrow 0
\end{aligned}
$$


Moreover, it is easy to see that the first term in the r.h.s. can be omitted: either projectile or target matrix element vanishes due to eq. (4.34). For the next two terms in the r.h.s. of eq. (6.38) we use

$$
\begin{aligned}
& \frac{1}{2 s^{3}}\left\{\left\langle\bar{\psi} A_{\nu}(x) \sigma_{\star k} \frac{1}{\alpha} \psi(0)\right\rangle_{A}\left\langle\bar{\psi} B^{i}(0) \sigma_{\bullet j} \gamma_{5} \frac{1}{\beta} \psi(x)\right\rangle_{B}\left(\delta_{i}^{k} \delta_{\mu}^{j}-\delta_{i}^{j} \delta_{\mu}^{k}\right)\right. \\
& \left.-\psi(0) \otimes \gamma_{5} \psi(x) \leftrightarrow \gamma_{5} \psi(0) \otimes \psi(x)\right\} \\
& =\frac{1}{2 s^{3}}\left\{\left\langle\bar{\psi}(x) A_{\nu}(x) \sigma_{\star}^{i} \gamma_{5} \frac{1}{\alpha} \psi(0)\right\rangle_{A}\left\langle\bar{\psi}\left[B_{\mu}(0) \sigma_{\bullet i}-B_{i}(0) \sigma_{\bullet \mu_{\perp}}\right] \frac{1}{\beta} \psi(x)\right\rangle_{B}\right. \\
& \quad-\left\langle\bar{\psi} A_{\nu}(x) \sigma_{\star \mu_{\perp}} \frac{1}{\alpha} \psi(0)\right\rangle_{A}\left\langle\bar{\psi} B^{j}(0) \sigma_{\bullet j} \gamma_{5} \frac{1}{\beta} \psi(x)\right\rangle_{B} \\
& \quad+\frac{s}{4}\left\langle\bar{\psi} A_{\nu}(x) \sigma_{m n} \gamma_{5} \frac{1}{\alpha} \psi(0)\right\rangle_{A}\left\langle\bar{\psi} B_{\mu}(x) \sigma^{m n} \frac{1}{\beta} \psi(x)\right\rangle_{B} \\
& \left.\quad-\frac{s}{2}\left\langle\bar{\psi}(x) A_{\nu}(x) \sigma_{\mu_{\perp}}^{j} \gamma_{5} \frac{1}{\alpha} \psi(0)\right\rangle_{A}\right\}\left\langle\bar{\psi} B_{i}(x) \sigma^{i j} \frac{1}{\beta} \psi(x)\right\rangle_{B}=0
\end{aligned}
$$

since the target matrix element in the first term vanishes due to Ee. (4.34), the one in the second term due to eq. (6.18), and the last two terms are small by power counting, $\sim \frac{q_{\perp}^{2}}{s}\left(\frac{q_{\mu}^{\perp} q_{\nu}^{\perp}}{Q_{\|}^{2}}\right)$. Similarly,

$$
\begin{aligned}
& \frac{2}{s^{3}}\left\{\left\langle\bar{\psi} A^{i}(x) \sigma_{\star j} \frac{1}{\alpha} \psi(0)\right\rangle_{A}\left\langle\bar{\psi} B_{\mu}(0) \sigma_{\bullet k} \gamma_{5} \frac{1}{\beta} \psi(x)\right\rangle_{B}\left(\delta_{i}^{k} \delta_{\nu}^{j}-\delta_{\nu}^{k} \delta_{i}^{j}\right)\right. \\
& \left.-\psi(0) \otimes \gamma_{5} \psi(x) \leftrightarrow \gamma_{5} \psi(0) \otimes \psi(x)\right\} \\
& =\left\langle\bar{\psi}(x)\left[A_{i}(x) \sigma_{\star \nu}-A_{\nu} \sigma_{\star i} \frac{1}{\alpha} \psi(0)\right\rangle_{A}\left\langle\bar{\psi} B_{\mu}(0) \sigma_{\bullet}^{i} \gamma_{5} \frac{1}{\beta} \psi(x)\right\rangle_{B}\right. \\
& \quad+\left\langle\bar{\psi} A^{i}(x) \sigma_{\star i} \gamma_{5} \frac{1}{\alpha} \psi(0)\right\rangle_{A}\left\langle\bar{\psi} B_{\mu}(0) \sigma_{\bullet \nu_{\perp}} \frac{1}{\beta} \psi(x)\right\rangle_{B} \\
& \quad-\frac{s}{4}\left\langle\bar{\psi}(x) A_{\nu} \sigma_{m n} \frac{1}{\alpha} \psi(0)\right\rangle_{A}\left\langle\bar{\psi} B_{\mu}(0) \sigma^{m n} \gamma_{5} \frac{1}{\beta} \psi(x)\right\rangle_{B} \\
& \quad+\frac{s}{2}\left\langle\bar{\psi}(x) A^{i} \sigma_{i j} \frac{1}{\alpha} \psi(0)\right\rangle_{A}\left\langle\bar{\psi} B_{\mu}(0) \sigma_{\nu_{\perp}}^{j} \gamma_{5} \frac{1}{\beta} \psi(x)\right\rangle_{B}=0
\end{aligned}
$$

for the same reason: the projectile matrix element in the first term in the r.h.s. vanishes due to eq. (4.34), the one in the second term due to eq. (6.18), and the last two terms are small by power counting. Thus, we get the result that there is no contribution to $W_{\mu_{\perp}}^{2 \mathrm{I}(1)}(q)$ with our accuracy.

If both $\mu$ and $\nu$ are longitudinal, using formula

$$
\sigma_{\mu_{\|} \alpha} \sigma_{\star j} \otimes \sigma_{\nu_{\|}}^{\alpha} \sigma_{\bullet j}+\mu \leftrightarrow \nu=-2 s g_{\mu \nu}^{\|}\left(\sigma_{\star j} \otimes \sigma_{\bullet j}-\frac{1}{s} \sigma_{\bullet k} \sigma_{\star j} \otimes \sigma_{\star}^{k} \sigma_{\bullet j}\right)
$$

we get

$$
\begin{aligned}
\check{W}_{\mu \nu}^{2 \mathrm{I}(1)}(x)= & -\frac{g_{\mu \nu}^{\|}}{2 s^{2}}\left\{\left\langle\bar{\psi} A^{i}(x) \sigma_{\star j} \frac{1}{\alpha} \psi(0)\right\rangle_{A}\left\langle\bar{\psi} B^{j}(0) \sigma_{\bullet} \gamma_{5} \frac{1}{\beta} \psi(x)\right\rangle_{B}\right. \\
& \left.-\psi(0) \otimes \gamma_{5} \psi(x) \leftrightarrow \gamma_{5} \psi(0) \otimes \psi(x)\right\}-x \leftrightarrow 0
\end{aligned}
$$


where we omitted contribution from the second term in the r.h.s. of eq. (6.41) due to power counting coming from Eq, (A.9). Now, using eq. (A.35) one obtains

$$
\begin{aligned}
\check{W}_{\mu \nu}^{2 \mathrm{I}(1)}(x)= & -\frac{g_{\mu \nu}^{\|}}{2 s^{2}}\left\{\left\langle\bar{\psi}(x)\left[A_{i}(x) \sigma_{\star j}-A_{j}(x) \sigma_{\star i}\right] \frac{1}{\alpha} \psi(0)\right\rangle_{A}\left\langle\bar{\psi} B^{j}(0) \sigma_{\bullet i} \gamma_{5} \frac{1}{\beta} \psi(x)\right\rangle_{B}\right. \\
& \left.+\left\langle\bar{\psi} A^{i}(x) \sigma_{\star i} \frac{1}{\alpha} \psi(0)\right\rangle_{A}\left\langle\bar{\psi} B^{j}(0) \sigma_{\bullet j} \gamma_{5} \frac{1}{\beta} \psi(x)\right\rangle_{B}\right\}-x \leftrightarrow 0=0
\end{aligned}
$$

due to eqs. (4.34) and (6.18). Thus, we get

$$
\check{W}_{\mu \nu}^{2 \mathrm{I}(1)}(x)=0
$$

Similarly, one can demonstrate that the contribution to $\check{W}_{\mu \nu}^{\mathrm{I}}(x)$ of eq. (6.20) coming from $\bar{\Xi}_{A}$ and $\bar{\Xi}_{B}$ vanishes.

Let us now consider term coming from $\bar{\Xi}_{2}$ and $\Xi_{2}$. After separating color-singlet contributions, it takes the form

$$
\begin{aligned}
\check{W}_{\mu \nu}^{3 \mathrm{I}}(x)= & -\frac{1}{4 s^{3}}\left\{\left\langle\bar{\psi} A^{j}(x) \sigma_{\mu \xi} A^{i} \psi(0)\right\rangle_{A}\left\langle\left(\bar{\psi}_{B} \frac{1}{\beta}\right) \sigma_{\bullet i} \sigma_{\nu}{ }^{\xi} \sigma_{\bullet j} \gamma_{5} \frac{1}{\beta} \psi(x)\right\rangle_{B}\right. \\
& \left.+\left\langle\bar{\psi} A^{j}(x) \sigma_{\mu \xi} \gamma_{5} A^{i} \psi(0)\right\rangle_{A}\left\langle\left(\bar{\psi}_{B} \frac{1}{\beta}\right) \sigma_{\bullet i} \sigma_{\nu}{ }^{\xi} \sigma_{\bullet j} \frac{1}{\beta} \psi(x)\right\rangle_{B}\right\}-x \leftrightarrow 0
\end{aligned}
$$

The power counting for similar eq. (4.58) in section 4.3.2 shows that we need to take $\mu$ and $\nu$ both longitudinal:

$$
\begin{aligned}
\check{W}_{\mu \nu}^{3 \mathrm{II}}(x)= & -\frac{p_{1 \mu} p_{1 \nu}}{s^{5}}\left\{\left\langle\bar{\psi} A^{j}(x) \sigma_{\star k} A^{i} \psi(0)\right\rangle_{A}\left\langle\left(\bar{\psi}_{B} \frac{1}{\beta}\right) \sigma_{\bullet i} \sigma_{\star}{ }_{\star} \sigma_{\bullet j} \gamma_{5} \frac{1}{\beta} \psi(x)\right\rangle_{B}\right. \\
& \left.+\left\langle\bar{\psi} A^{j}(x) \sigma_{\star k} \gamma_{5} A^{i} \psi(0)\right\rangle_{A}\left\langle\left(\bar{\psi}_{B} \frac{1}{\beta}\right) \sigma_{\bullet i} \sigma_{\star}^{k} \sigma_{\bullet j} \frac{1}{\beta} \psi(x)\right\rangle_{B}\right\} \\
= & \frac{i p_{1 \mu} p_{1 \nu}}{s^{4}}\left\{\left\langle\bar{\psi} A^{j}(x) \sigma_{\star k} A^{i} \psi(0)\right\rangle_{A}\left\langle\left(\bar{\psi}_{B} \frac{1}{\beta}\right) \gamma_{i} \sigma_{\bullet}{ }^{k} \gamma_{j} \gamma_{5} \frac{1}{\beta} \psi(x)\right\rangle_{B}\right. \\
& \left.-\psi(0) \otimes \gamma_{5} \psi(x) \leftrightarrow \gamma_{5} \psi(0) \otimes \psi(x)\right\}-x \leftrightarrow 0 \\
= & \frac{i p_{1 \mu} p_{1 \nu}}{s^{4}}\left\{\left\langle\bar{\psi}(x) \not A(x) \sigma_{\star k} A(0) \psi(0)\right\rangle_{A}\left\langle\left(\bar{\psi}_{B} \frac{1}{\beta}\right) \sigma_{\bullet}{ }^{k} \gamma_{5} \frac{1}{\beta} \psi(x)\right\rangle_{B}=0\right.
\end{aligned}
$$

where we used formula $\sigma_{\star k} \otimes \sigma_{\bullet}{ }^{k} \gamma_{5}-\sigma_{\star k} \gamma_{5} \otimes \sigma_{\bullet}{ }^{k}=0$ following from eq. (A.13). Also, one can demonstrate that the contribution to $\check{W}_{\mu \nu}^{\mathrm{I}}$ coming from $\bar{\Xi}_{A}$ and $\Xi_{A}$ vanishes. Finally, similarly to eq. (4.63), we can neglect terms $\sim \frac{1}{N_{c}^{2}}$ coming from $\bar{\Xi}_{1}, \Xi_{B}$ and $\bar{\Xi}_{2}, \Xi_{A}$, see the discussion in section 4.3.2. Thus, we get the result that the contribution to $\check{W}_{\mu \nu}^{\mathrm{I}}$ coming from two quark-quark-gluon TMDs vanishes with our accuracy:

$$
\check{W}_{\mu \nu}^{2 \mathrm{I}}=0
$$




\subsubsection{Exchange-type power corrections}

The exchange-type power corrections to eq. (6.13) are

$$
\begin{aligned}
\check{W}_{\mu \nu}^{\mathrm{I} 2 \operatorname{Sex}}(x)=\frac{N_{c}}{2 s} & \sum_{f, f^{\prime}}\langle A, B|\left(\left(e_{f^{\prime}} c_{f} a_{f}-e_{f} c_{f^{\prime}} a_{f^{\prime}}\right)\left[\bar{\Psi}_{1}(x) \gamma_{\mu} \Psi_{1}(x)\right]^{f}\left[\bar{\Psi}_{2}(0) \gamma_{\nu} \Psi_{2}(0)\right]^{f^{\prime}}\right. \\
& -e_{f^{\prime}} c_{f}\left[\bar{\Psi}_{1}(x) \gamma_{\mu} \gamma_{5} \Psi_{1}(x)\right]^{f}\left[\bar{\Psi}_{2}(0) \gamma_{\nu} \Psi_{2}(0)\right]^{f^{\prime}} \\
& \left.+e_{f} c_{f^{\prime}}\left[\bar{\Psi}_{1}(x) \gamma_{\mu} \Psi_{1}(x)\right]^{f}\left[\bar{\Psi}_{2}(0) \gamma_{\nu} \gamma_{5} \Psi_{2}(0)\right]^{f^{\prime}}|A, B\rangle\right)+\mu \leftrightarrow \nu-x \leftrightarrow 0
\end{aligned}
$$

The terms in the r.h.s. differ from those in eqs. (4.70), (5.14), and (5.15) by replacement of " $+x \leftrightarrow 0$ " by " $-x \leftrightarrow 0$ " which leads to change sign of " \pm c.c." terms in those equations so we get instead of eq. (5.21)

$$
\begin{aligned}
W_{\mu \nu}^{\mathrm{I} 2 \operatorname{Sex}}(q)= & \frac{i N_{c}}{2\left(N_{c}^{2}-1\right) Q_{\|}^{2}} \sum_{f, f^{\prime}} \int d^{2} k_{\perp}\left\{( e _ { f } c _ { f ^ { \prime } } a _ { f ^ { \prime } } - e _ { f ^ { \prime } } c _ { f } a _ { f } ) \left[k_{\mu}^{\perp}(q-k)_{\nu}^{\perp}+k_{\nu}^{\perp}(q-k)_{\mu}^{\perp}\right.\right. \\
& \left.+g_{\mu \nu}^{\perp}(k, q-k)_{\perp}\right] I_{--}^{1 f f^{\prime}}\left(q, k_{\perp}\right)-g_{\mu \nu}^{\perp}(k, q-k)_{\perp} I_{--}^{2 f f^{\prime}}\left(q, k_{\perp}\right) \\
& +c_{f} e_{f^{\prime}}\left[\epsilon_{\mu m} k^{m}(q-k)_{\nu}+\mu \leftrightarrow \nu\right] J_{+-}^{1 f f^{\prime}}\left(q, k_{\perp}\right) \\
& \left.-c_{f^{\prime}} e_{f}\left[k_{\mu} \epsilon_{\nu n}(q-k)^{n}+\mu \leftrightarrow \nu\right] J_{-+}^{1 f f^{\prime}}\left(q, k_{\perp}\right)\right\}
\end{aligned}
$$

where $J_{ \pm \pm}^{i}$ and $I_{--}^{i}$ are defined in eqs. (A.71) and (A.72).

\subsubsection{Result for symmetric interference term $W^{\mathrm{I} 2 \mathrm{san}}$}

As usual, we represent the result for hadronic tensor $W_{\mu \nu}^{\mathrm{I} 2 \mathrm{~S}}(q)$ as a sum of the "annihilation" and "exchange" parts:

$$
W_{\mu \nu}^{\mathrm{I} 2 \mathrm{~S}}(q)=W_{\mu \nu}^{\mathrm{I} 2 \operatorname{San}}(q)+W_{\mu \nu}^{\mathrm{I} 2 \operatorname{Sex}}(q)
$$

where the exchange-type corrections are presented in eq. (6.49) above while $W_{\mu \nu}^{\mathrm{I} 2 \mathrm{San}}(q)$ is given by the sum of eqs. (6.22) and (6.35)

$$
\begin{aligned}
W_{\mu \nu}^{\mathrm{I} 2 \operatorname{San}}(q)= & \sum_{f} e_{f} c_{f} W_{\mu \nu}^{\mathrm{If}}(q) \\
W_{\mu \nu}^{\mathrm{If}}(q)= & -\frac{i \epsilon_{\mu j}}{2 m^{2}} \int d^{2} k_{\perp}\left(\left[k^{j}(q-k)_{\nu}^{\perp}+(q-k)_{\perp}^{j} k_{\nu}^{\perp}\right]\right. \\
& \left.+\frac{4}{Q_{\|}^{2}}\left[\beta p_{2 \nu} k^{j}(q-k)_{\perp}^{2}+\alpha p_{1 \nu}(q-k)^{j} k_{\perp}^{2}\right]\right) \mathcal{H}^{f}\left(q, k_{\perp}\right)+\mu \leftrightarrow \nu
\end{aligned}
$$

Let us check gauge invariance of annihilation part of interference hadronic tensor 
$q^{\mu} W_{\mu \nu}^{\mathrm{I} 2 \mathrm{San}}(q)=0$. First, let us rewrite it in as follows:

$$
\begin{aligned}
W_{\mu \nu}^{\mathrm{If}}(q)= & -\frac{i \epsilon_{\mu j}}{2 m^{2}}\left(\delta_{\nu}^{i}-2 \frac{q_{\nu}^{\|} q^{i}}{Q_{\|}^{2}}\right) \int d^{2} k_{\perp}\left[k^{j}(q-k)_{i}+(q-k)^{j} k_{i}+\delta_{i}^{j}(k, q-k)_{\perp}\right] \mathcal{H}^{f}\left(q, k_{\perp}\right) \\
& +\frac{i \epsilon_{\mu j}}{m^{2}} \frac{\tilde{q}_{\nu}}{Q_{\|}^{2}} \int d^{2} k_{\perp}\left[k^{j}(q-k)_{\perp}^{2}-(q-k)^{j} k_{\perp}^{2}\right] \mathcal{H}^{f}\left(q, k_{\perp}\right)+\mu \leftrightarrow \nu
\end{aligned}
$$

The integral in the first term is proportional to $\left(2 q_{i} q^{j}+\delta_{i}^{q} q_{\perp}^{2}\right)$ and it is easy to see that

$$
q^{\mu}\left[\epsilon_{\mu j}\left(\delta_{\nu}^{i}-2 \frac{q_{\nu}^{\|} q^{i}}{Q_{\|}^{2}}\right)\left(2 q_{i} q^{j}+\delta_{i}^{j}\right)+\mu \leftrightarrow \nu\right]=0
$$

The integral in the second term is proportional to $q^{j}$ so

$$
q^{\mu}\left[\epsilon_{\mu j} q^{j}+\mu \leftrightarrow \nu\right]=0
$$

and therefore $q^{\mu} W_{\mu \nu}^{\text {If }}(q)=0$.

\subsection{Antisymmetric interference term of tensor $W^{\mathrm{I} 2}$}

Similarly to the symmetric case (6.13), from definitions (2.6) and (2.9) we get

$$
\begin{aligned}
\check{W}_{\mu \nu}^{\mathrm{I} 2 \mathrm{~A}}(x)= & \frac{N_{c}}{2 s} \sum_{f, f^{\prime}}\left(\left(e_{f^{\prime}} c_{f} a_{f}-e_{f} c_{f^{\prime}} a_{f^{\prime}}\right)\langle A, B|\left[\bar{\psi}(x) \gamma_{\mu} \psi(x)\right]^{f}\left[\bar{\psi}(0) \gamma_{\nu} \psi(0)\right]^{f^{\prime}}\right. \\
& +e_{f} c_{f^{\prime}}\langle A, B|\left[\bar{\psi}(x) \gamma_{\mu} \psi(x)\right]^{f}\left[\bar{\psi}(0) \gamma_{\nu} \gamma_{5} \psi(0)\right]^{f^{\prime}} \\
& \left.-e_{f^{\prime}} c_{f}\left\langle A, B\left|\left[\bar{\psi}(x) \gamma_{\mu} \gamma_{5} \psi(x)\right]^{f}\left[\bar{\psi}(0) \gamma_{\nu} \psi(0)\right]^{f^{\prime}}\right| A, B\right\rangle\right)-\mu \leftrightarrow \nu
\end{aligned}
$$

\subsubsection{Annihilation-type power corrections}

Again, let us start with annihilation-type power corrections. Since in this case $f=f^{\prime}$, the first term in the r.h.s. of eq. (6.55) vanishes and the second can be written as

$$
\begin{aligned}
\check{W}_{\mu \nu}^{\mathrm{I} 2 \mathrm{Aa}}(x)= & \sum_{f} e_{f} c_{f} \check{W}_{\mu \nu}^{\mathrm{IAf}}(x) \\
\check{W}_{\mu \nu}^{\mathrm{IAf}}(x)= & \frac{N_{c}}{2 s}\langle A, B|\left[\bar{\Psi}_{1}(x) \gamma_{\mu} \bar{\Psi}_{2}(x)\right]\left[\bar{\Psi}_{2}(0) \gamma_{\nu} \gamma_{5} \bar{\Psi}_{1}(0)\right] \\
& -\left[\bar{\Psi}_{1}(x) \gamma_{\mu} \gamma_{5} \bar{\Psi}_{2}(x)\right]\left[\bar{\Psi}_{2}(0) \gamma_{\nu} \bar{\Psi}_{1}(0)\right]-\mu \leftrightarrow \nu|A, B\rangle+x \leftrightarrow 0
\end{aligned}
$$

where we made the usual replacement $\psi \rightarrow \Psi_{1}+\Psi_{2}$. After Fierz transformation (A.6) the r.h.s. of the above equation turns to

$$
\begin{aligned}
& \check{W}_{\mu \nu}^{\mathrm{IA}}(x) \\
& =i \frac{N_{c}}{2 s}\left\langle p_{A}, p_{B}\right|\left[\bar{\Psi}_{1}^{m}(x) \Psi_{1}^{n}(0)\right]\left[\bar{\Psi}_{2}^{n}(0) \sigma_{\mu \nu} \gamma_{5} \Psi_{2}^{m}(x)\right]-\left[\bar{\Psi}_{1}^{m}(x) \sigma_{\mu \nu} \Psi_{1}^{n}(0)\right]\left[\bar{\Psi}_{2}^{n}(0) \gamma_{5} \Psi_{2}^{m}(x)\right] \\
& -\left[\bar{\Psi}_{1}^{m}(x) \gamma_{5} \Psi_{1}^{n}(0)\right]\left[\bar{\Psi}_{2}^{n}(0) \sigma_{\mu \nu} \Psi_{1}^{m}(x)\right]+\left[\bar{\Psi}_{1}^{m}(x) \sigma_{\mu \nu} \gamma_{5} \Psi_{1}^{n}(0)\right]\left[\bar{\Psi}_{2}^{n}(0) \Psi_{1}^{m}(x)\right]\left|p_{A}, p_{B}\right\rangle+x \leftrightarrow 0
\end{aligned}
$$

where we suppressed flavor label. 
Let's us demonstrate that $\check{W}_{\mu \nu}^{\mathrm{IA}}(x)$ is small in our approximation. After Fierz transformation (A.6) one obtains

$$
\begin{aligned}
& \check{W}_{\mu \nu}^{\mathrm{IA}}(x) \\
& =\frac{i N_{c}}{2 s}\langle A, B|-\left[\bar{\Psi}_{1}^{m}(x) \gamma_{5} \Psi_{1}^{n}(0)\right]\left[\bar{\Psi}_{2}^{n}(0) \sigma_{\mu \nu} \Psi_{2}^{m}(x)\right]+\left[\bar{\Psi}_{1}^{m}(x) \sigma_{\mu \nu} \gamma_{5} \Psi_{1}^{n}(0)\right]\left[\bar{\Psi}_{2}^{n}(0) \Psi_{2}^{m}(x)\right] \\
& \quad+\left[\bar{\Psi}_{1}^{m}(x) \Psi_{1}^{n}(0)\right]\left[\bar{\Psi}_{2}^{n}(0) \sigma_{\mu \nu} \gamma_{5} \Psi_{2}^{m}(x)\right]-\left[\bar{\Psi}_{1}^{m}(x) \sigma_{\mu \nu} \Psi_{1}^{n}(0)\right]\left[\bar{\Psi}_{2}^{n}(0) \gamma_{5} \Psi_{2}^{m}(x)\right]|A, B\rangle+x \leftrightarrow 0
\end{aligned}
$$

It is convenient to convolute $W_{\mu \nu}^{\mathrm{IA}}(x)$ with $\epsilon_{\mu \nu \alpha \beta}$ and consider

$$
\mathcal{W}_{\mu \nu}^{\mathrm{IA}}(x)=\frac{i}{2} \epsilon_{\mu \nu}^{\alpha \beta} W_{\alpha \beta}^{\mathrm{IA}}(x)
$$

then

$$
\begin{aligned}
& \check{\mathcal{W}}_{\mu \nu}^{\mathrm{Ia}}(x) \\
& =\frac{i N_{c}}{2 s}\langle A, B|-\left[\bar{\Psi}_{1}^{m}(x) \gamma_{5} \Psi_{1}^{n}(0)\right]\left[\bar{\Psi}_{2}^{n}(0) \sigma_{\mu \nu} \gamma_{5} \Psi_{2}^{m}(x)\right]+\left[\bar{\Psi}_{1}^{m}(x) \sigma_{\mu \nu} \Psi_{1}^{n}(0)\right]\left[\bar{\Psi}_{2}^{n}(0) \Psi_{2}^{m}(x)\right] \\
& \quad+\left[\bar{\Psi}_{1}^{m}(x) \Psi_{1}^{n}(0)\right]\left[\bar{\Psi}_{2}^{n}(0) \sigma_{\mu \nu} \Psi_{2}^{m}(x)\right]-\left[\bar{\Psi}_{1}^{m}(x) \sigma_{\mu \nu} \gamma_{5} \Psi_{1}^{n}(0)\right]\left[\bar{\Psi}_{2}^{n}(0) \gamma_{5} \Psi_{2}^{m}(x)\right]|A, B\rangle+x \leftrightarrow 0
\end{aligned}
$$

This is similar to $\check{W}_{\mu \nu}^{\text {as }}(x)$ of eq. (5.56) studied in previous section. The only difference is the relative sign between the first and the second term in the r.h.s. of these equations (and replacement of " $-x \leftrightarrow 0$ " by " $+x \leftrightarrow 0$ " which does not change power counting). Let us qiuckly check that this relative sign does not change the result that the contribution is small. First, let us consider the one-gluon contribution similar to eq. (5.57)

$$
\begin{aligned}
& \frac{i}{s^{2}}\left\{\left\langle\bar{\psi}(x) \not \not_{2} \gamma_{i} \frac{1}{\alpha} \psi(0)\right\rangle_{A}\left\langle\bar{\psi}(0) B^{i}(0) \sigma_{\mu \nu} \psi(x)\right\rangle_{B}\right. \\
& \left.+\left\langle\bar{\psi}(x) \sigma_{\mu \nu} \not \not_{2} \gamma^{i} \frac{1}{\alpha} \psi(0)\right\rangle_{A}\left\langle\bar{\psi}(0) B_{i}(0) \psi(x)\right\rangle_{B}-\psi(0) \otimes \psi(x) \leftrightarrow \gamma_{5} \psi(0) \otimes \gamma_{5} \psi(x)\right\}-x \leftrightarrow 0
\end{aligned}
$$

As discussed in previous section after eq. (5.57), the two terms in the r.h.s. of eq. (5.57) vanish separately for transverse $\mu$ and $\nu$, are both small for one longitudinal and one transverse index, and the term which changed sign is neglected in eq. (5.59) so in all cases the relative sign does not matter. Similarly, one can check this for other one-gluon terms.

Let us now consider two-gluon term coming from $\Xi_{1}$ and $\Xi_{2}$

$$
\begin{aligned}
& \frac{i}{2 s^{3}}\left\{\left\langle\bar{\psi} A^{j}(x) \sigma_{\star i} \frac{1}{\alpha} \psi(0)\right\rangle_{A}\left\langle\bar{\psi} B^{i}(0) \sigma_{\mu \nu} \sigma_{\bullet j} \frac{1}{\beta} \psi(x)\right\rangle_{B}+\left\langle\bar{\psi} A^{j}(x) \sigma_{\mu \nu} \sigma_{\star i} \frac{1}{\alpha} \psi(0)\right\rangle_{A}\right. \\
& \left.\times\left\langle\bar{\psi} B^{i}(0) \sigma_{\bullet j} \frac{1}{\beta} \psi(x)\right\rangle_{B}-\psi(0) \otimes \psi(x) \leftrightarrow \gamma_{5} \psi(0) \otimes \gamma_{5} \psi(x)\right\}+x \leftrightarrow 0
\end{aligned}
$$


Again, this differs from eq. (5.65) by relative sign between two terms. Looking at the derivation of eq. (5.67) we see that both terms in the r.h.s. vanish separately due to eq. (4.34) so

$$
\begin{aligned}
\text { eq. }(6.62)= & \frac{1}{2 s^{3}}\left\{\left\langle\bar{\psi}\left[A_{\mu}(x) \sigma_{\star \nu_{\perp}}-\mu \leftrightarrow \nu\right] \frac{1}{\alpha} \psi(0)\right\rangle_{A}\left\langle\bar{\psi} B^{i}(0) \sigma_{\bullet i} \frac{1}{\beta} \psi(x)\right\rangle_{B}\right. \\
& \left.+\left\langle\bar{\psi} A^{i}(x) \sigma_{\star i} \frac{1}{\alpha} \psi(0)\right\rangle_{A}\left\langle\bar{\psi}\left[B_{\mu}(0) \sigma_{\bullet \nu_{\perp}}-\mu \leftrightarrow \nu\right] \frac{1}{\beta} \psi(x)\right\rangle_{B}\right\}+x \leftrightarrow 0=0
\end{aligned}
$$

If $\mu$ and $\nu$ are longitudinal, we get

$$
\begin{aligned}
\text { eq. }(6.62)= & \frac{2 i}{s^{5}}\left(p_{1 \mu} p_{2 \nu}-\mu \leftrightarrow \nu\right)\left\{\left\langle\bar{\psi} A^{j}(x) \sigma_{\star i} \frac{1}{\alpha} \psi(0)\right\rangle_{A}\left\langle\bar{\psi} B^{i}(0) \sigma_{\star \bullet} \sigma_{\bullet j} \frac{1}{\beta} \psi(x)\right\rangle_{B}\right. \\
& +\left\langle\bar{\psi} A^{j}(x) \sigma_{\star \bullet} \sigma_{\star i} \frac{1}{\alpha} \psi(0)\right\rangle_{A}\left\langle\bar{\psi} B^{i}(0) \sigma_{\bullet j} \frac{1}{\beta} \psi(x)\right\rangle_{B} \\
& \left.-\psi(0) \otimes \psi(x) \leftrightarrow \gamma_{5} \psi(0) \otimes \gamma_{5} \psi(x)\right\}-x \leftrightarrow 0=0
\end{aligned}
$$

If now one of indices is longitudinal and the other transverse, the contribution is small due to power counting as discussed after eq. (5.71) so the two-gluon term coming from $\Xi_{1}$ and $\Xi_{2}$ vanishes with our accuracy. The corresponding contribution coming from $\bar{\Xi}_{1}$ and $\bar{\Xi}_{2}$ vanishes for the same reason.

Finally, as shown in eq. (5.72), terms coming from $\bar{\Xi}_{1}, \Xi_{1}$ and from $\bar{\Xi}_{2 B}, \Xi_{2 B}$ are small due to power counting. Also, as usually we neglect the terms coming from $\bar{\Xi}_{1}, \Xi_{2 B}$ and from $\bar{\Xi}_{2 B}, \Xi_{1}$ are $\sim \frac{1}{N_{c}^{2}}$ so

$$
\check{\mathcal{W}}_{\mu \nu}^{\mathrm{IA}}(q)=\check{W}_{\mu \nu}^{\mathrm{IA}}(q)=0
$$

with our accuracy.

\subsubsection{Exchange-type power corrections and the result for $W^{\mathrm{I} 2}$}

The exchange-type power corrections to eq. (6.55) are

$$
\begin{aligned}
\left(\check{W}^{\mathrm{ex}}\right)_{\mu \nu}^{\mathrm{I} 2 \mathrm{~A}}(x)= & \frac{N_{c}}{2 s} \sum_{f, f^{\prime}}\langle A, B|\left(\left(e_{f^{\prime}} c_{f} a_{f}-e_{f} c_{f^{\prime}} a_{f^{\prime}}\right)\left[\bar{\Psi}_{1}(x) \gamma_{\mu} \Psi_{1}(x)\right]^{f}\left[\bar{\Psi}_{2}(0) \gamma_{\nu} \Psi_{2}(0)\right]^{f^{\prime}}\right. \\
& +e_{f} c_{f^{\prime}}\left[\bar{\Psi}_{1}(x) \gamma_{\mu} \Psi_{1}(x)\right]^{f}\left[\bar{\Psi}_{2}(0) \gamma_{\nu} \gamma_{5} \Psi_{2}(0)\right]^{f^{\prime}} \\
& \left.-e_{f^{\prime}} c_{f}\left[\bar{\Psi}_{1}(x) \gamma_{\mu} \gamma_{5} \Psi_{1}(x)\right]^{f}\left[\bar{\Psi}_{2}(0) \gamma_{\nu} \Psi_{2}(0)\right]^{f^{\prime}}|A, B\rangle\right)-\mu \leftrightarrow \nu+x \leftrightarrow 0
\end{aligned}
$$

Similarly to the symmetric case, one can use formulas from section 5.3.3 with change of signs of complex conjugations. Indeed, the terms in the r.h.s. of eq. (6.66) differ from those in eqs. (5.76) and (5.82) by replacement of " $+x \leftrightarrow 0$ " by " $-x \leftrightarrow 0$ " which leads to change sign of " \pm c.c." terms in those equations. We get

$$
\begin{aligned}
\left(W^{\mathrm{I} 2 \mathrm{~A}}\right)_{\mu \nu}^{\mathrm{ex}}(q)= & -\frac{N_{c}}{\left(N_{c}^{2}-1\right) Q_{\|}^{2}} \sum_{f, f^{\prime}} c_{f} c_{f^{\prime}} \int d^{2} k_{\perp}\left\{a_{f}\left[\epsilon_{\mu m} k^{m}(q-k)_{\nu}-\mu \leftrightarrow \nu\right] I_{+-}^{2 f f^{\prime}}\left(q, k_{\perp}\right)\right. \\
& \left.-a_{f^{\prime}}\left[k_{\mu} \epsilon_{\nu n}(q-k)^{n}-\mu \leftrightarrow \nu\right] I_{-+}^{2 f f^{\prime}}\left(q, k_{\perp}\right)\right\}
\end{aligned}
$$

cf. eq. (5.85). As usually, exchange power corrections exist only for transverse $\mu$ and $\nu$. 
Finally, since we proved in previous section that annihilation-type power corrections $W_{\mu \nu}^{\mathrm{I} 2 \mathrm{~A}}$ vanish, the total result for $W_{\mu \nu}^{\mathrm{I} 2 \mathrm{~A}}$ is equal to the "exchange" part:

$$
W_{\mu \nu}^{\mathrm{I} 2 \mathrm{~A}}(q)=\left(W^{\mathrm{I} 2 \mathrm{~A}}\right)_{\mu \nu}^{\mathrm{ex}}(q)=\text { r.h.s of eq. }(6.67)
$$

\section{Results}

In this section we will take into account only gauge-invariant terms coming from annihilation-type terms proportional to TMDs $f_{1}$ and $h_{1}^{\perp}$. The reason to neglect annihilation-type $\sim W^{2 \mathrm{H}}(q)$ is that the twist-three matrix elements (A.63) are virtually unknown, and exchange-type power corrections can presumably be neglected due to extra $\frac{1}{N_{c}}$. Anyway, taking into account leading-twist contributions and their "gauge-invariancerestoring" counterparts appears to a good start for estimations of DY hadronic tensors.

\subsection{Hadronic tensors in Collins-Soper frame}

In Collins-Soper frame the hadronic tensors are parametrized in terms of $q$ and three unit vectors $X, Y, Z$ orthogonal to $q$ and to each other. In terms of Sudakov variables they are

$$
\begin{aligned}
Z & =\frac{\tilde{q}}{Q_{\|}}=\frac{1}{Q_{\|}}\left(\alpha_{q} p_{1}-\beta_{q} p_{2}\right), \quad X=\left(\frac{Q_{\perp}}{Q_{\|} Q} q+\frac{Q}{Q_{\perp} Q_{\|}} q_{\perp}\right) \\
Y_{\mu} & =-\frac{1}{Q} \epsilon_{\mu \nu \lambda \rho} X^{\nu} Z^{\lambda} q^{\rho}=\epsilon_{\mu i} \frac{q_{\perp}^{i}}{Q_{\perp}}
\end{aligned}
$$

where $Q_{\perp} \equiv\left|q_{\perp}\right|$.

\subsubsection{Hadronic tensor for photon-mediated DY process}

We parametrize photon-mediated hadronic tensor in a standard way (up to extra $N_{c}$ and flavor factors), separately for $W_{\mu \nu}^{\mathrm{F} f}$ and $W_{\mu \nu}^{\mathrm{H} f}$ defined in eq. (4.79)

$$
\begin{aligned}
W_{\mu \nu}^{\mathrm{F} f}(q)= & -\left(g_{\mu \nu}-\frac{q_{\mu} q_{\nu}}{q^{2}}\right)\left(W_{T}^{\mathrm{F} f}+W_{\Delta \Delta}^{\mathrm{F} f}\right)-2 X_{\mu} X_{\nu} W_{\Delta \Delta}^{\mathrm{F} f} \\
& +Z_{\mu} Z_{\nu}\left(W_{L}^{\mathrm{F} f}-W_{T}^{\mathrm{F} f}-W_{\Delta \Delta}^{\mathrm{F} f}\right)-\left(X_{\mu} Z_{\nu}+X_{\nu} Z_{\mu}\right) W_{\Delta}^{\mathrm{F} f}
\end{aligned}
$$

and similarly

$$
\begin{aligned}
W_{\mu \nu}^{\mathrm{H} f}(q)= & -\left(g_{\mu \nu}-\frac{q_{\mu} q_{\nu}}{q^{2}}\right)\left(W_{T}^{\mathrm{H} f}+W_{\Delta \Delta}^{\mathrm{H} f}\right)-2 X_{\mu} X_{\nu} W_{\Delta \Delta}^{\mathrm{H} f} \\
& +Z_{\mu} Z_{\nu}\left(W_{L}^{\mathrm{H} f}-W_{T}^{\mathrm{H} f}-W_{\Delta \Delta}^{\mathrm{H} f}\right)-\left(X_{\mu} Z_{\nu}+X_{\nu} Z_{\mu}\right) W_{\Delta}^{\mathrm{H} f}
\end{aligned}
$$

The expressions for $W_{i}(q)$ can be easily obtained from eqs. (4.30) and (4.66):

$$
\begin{array}{ll}
W_{T}^{\mathrm{F} f}(q)=\int d^{2} k_{\perp}\left[1-\frac{q_{\perp}^{2}}{2 Q_{\|}^{2}}\right] F^{f}\left(q, k_{\perp}\right), & W_{L}^{\mathrm{F} f}(q)=\int d k_{\perp} \frac{(q-2 k)_{\perp}^{2}}{Q_{\|}^{2}} F^{f}\left(q, k_{\perp}\right) \\
W_{\Delta \Delta}^{\mathrm{F} f}(q)=\int d^{2} k_{\perp} \frac{q_{\perp}^{2}}{2 Q_{\|}^{2}} F^{f}\left(q, k_{\perp}\right), & W_{\Delta}^{\mathrm{F} f}(q)=\frac{Q}{Q_{\|}^{2} Q_{\perp}} \int d^{2} k_{\perp}(q, q-2 k)_{\perp} F^{f}\left(q, k_{\perp}\right)
\end{array}
$$


and

$$
\begin{aligned}
W_{T}^{\mathrm{H} f}(q)= & \frac{1}{2 m^{2} Q^{2}} \int d^{2} k_{\perp}\left(2 k_{\perp}^{2}(q-k)_{\perp}^{2}+\left[k_{\perp}^{2}+(q-k)_{\perp}^{2}\right](k, q-k)_{\perp}\right) H^{f}\left(q, k_{\perp}\right), \\
W_{L}^{\mathrm{H} f}(q)= & \frac{1}{m^{2} Q^{2}} \int d k_{\perp}\left(2 k_{\perp}^{2}(q-k)_{\perp}^{2}-\left[k_{\perp}^{2}+(q-k)_{\perp}^{2}\right](k, q-k)_{\perp}\right) H^{f}\left(q, k_{\perp}\right) \\
W_{\Delta \Delta}^{\mathrm{H} f}(q)= & \frac{1}{m^{2}} \int d^{2} k_{\perp}\left(\frac{2(q, k)_{\perp}(q, q-k)_{\perp}}{q_{\perp}^{2}}-(k, q-k)_{\perp}+\frac{1}{Q^{2}}\left\{k_{\perp}^{2}(q-k)_{\perp}^{2}\right.\right. \\
& \left.\left.+\frac{1}{2}(k, q-k)_{\perp}\left[k_{\perp}^{2}+(q-k)_{\perp}^{2}\right]+q_{\perp}^{2}(k, q-k)_{\perp}-2(q, k)(q, q-k)_{\perp}\right\}\right) H^{f}\left(q, k_{\perp}\right) \\
W_{\Delta}^{\mathrm{H} f}(q)= & -\frac{Q}{Q_{\|}^{2} Q_{\perp} m^{2}} \int d^{2} k_{\perp}(q, q-2 k)(k, q-k)_{\perp} H^{f}\left(q, k_{\perp}\right)
\end{aligned}
$$

These expressions were obtained in ref. [16].

\subsubsection{Hadronic tensor for $Z$-mediated DY process}

The symmetric part of $W_{\mu \nu}^{\mathrm{ZS}}$ is given by eq. (5.19)

$$
W_{\mu \nu}^{\mathrm{ZS}}=e^{2} \sum_{f} c_{f}^{2}\left[\left(a_{f}^{2}+1\right) W_{\mu \nu}^{f \mathrm{~F}}(q)+\left(a_{f}^{2}-1\right) W_{\mu \nu}^{f \mathrm{H}}(q)\right]
$$

where $W_{\mu \nu}^{\mathrm{Ff}}(q)$ and $W_{\mu \nu}^{\mathrm{Hf}}(q)$ are expressed in terms of $X$ and $Z$ vectors in eqs. (7.3) and (7.4) above.

The antisymmetric part (5.83) can be parametrized as

$$
W_{\mu \nu}^{\mathrm{ZA}}(q)=-2 i \epsilon_{\mu \nu \lambda \rho} q^{\lambda} \sum_{f} a_{f} c_{f}^{2}\left[\frac{Z^{\rho}}{Q_{\|}} \mathcal{W}_{4}^{\mathrm{F} f}(q)+X^{\rho} \frac{Q_{\perp}}{Q_{\|} Q} \mathcal{W}_{3}^{\mathrm{F} f}(q)\right]
$$

where

$$
\mathcal{W}_{4}^{\mathrm{F} f}(q)=\int d^{2} k_{\perp} \mathcal{F}^{f}\left(q, k_{\perp}\right), \quad \mathcal{W}_{3}^{\mathrm{F} f}(q)=\int d^{2} k_{\perp}\left(1-\frac{2(q, k)_{\perp}}{q_{\perp}^{2}}\right) \mathcal{F}^{f}\left(q, k_{\perp}\right)
$$

with $\mathcal{F}^{f}\left(q, k_{\perp}\right)$ given by eq. (5.27).

\subsubsection{Interference tensors}

The symmetric part of the interference tensor $W_{\mu \nu}^{\mathrm{I1}}(q)$ is given by eq. (6.6)

$$
W_{\mu \nu}^{\mathrm{I1S}}(q)=\sum_{f} e_{f} c_{f} a_{f}\left[W_{\mu \nu}^{\mathrm{Ff}}(q)+W_{\mu \nu}^{\mathrm{Hf}}(q)\right]
$$

where $W_{\mu \nu}^{\mathrm{F} f}(q)$ and $W_{\mu \nu}^{\mathrm{Hf}}(q)$ are given by eqs. (7.3) and (7.4) above.

The antisymmetric part of $W_{\mu \nu}^{\mathrm{I} 1}(q)$ is given by eq. (6.12) which can be represented similarly to eq. (7.14)

$$
W_{\mu \nu}^{\mathrm{I1 \textrm {A }}}(q)=-i \epsilon_{\mu \nu \lambda \rho} q^{\lambda} \sum_{f} e_{f} c_{f}\left[\frac{Z^{\rho}}{Q_{\|}} \mathcal{W}_{A}^{\mathrm{Ff}}(q)+X^{\rho} \frac{Q_{\perp}}{Q_{\|} Q} \mathcal{W}_{B}^{\mathrm{F} f}(q)\right]
$$


where $\mathcal{W}_{A, B}^{\mathrm{Ff}}(q)$ are given by eq. (7.9).

Next, the symmetric part of $W_{\mu \nu}^{\mathrm{I} 2}(q)$, given by eq. (6.52), can be parametrized as

$$
W_{\mu \nu}^{\mathrm{If}}(q)=i \sum_{f} e_{f} c_{f}\left(\frac{Q}{Q_{\|}} Y_{\mu} X_{\nu} W_{1}^{\mathrm{If}}(q)+Y_{\mu} Z_{\nu} \frac{Q_{\perp}}{Q_{\|}} W_{2}^{\mathrm{If}}(q)\right)+\mu \leftrightarrow \nu
$$

where

$$
\begin{aligned}
& W_{1}^{\text {If }}(q)=\frac{1}{m^{2}} \int d^{2} k_{\perp}\left[\frac{2(q, k)_{\perp}(q, q-k)_{\perp}}{q_{\perp}^{2}}-(k, q-k)_{\perp}\right] \mathcal{H}^{f}\left(q, k_{\perp}\right) \\
& W_{2}^{\text {If }}(q)=\frac{1}{m^{2} q_{\perp}^{2}} \int d^{2} k_{\perp}\left[(q, k)_{\perp}(q-k)_{\perp}^{2}-(q, q-k)_{\perp} k_{\perp}^{2}\right] \mathcal{H}^{f}\left(q, k_{\perp}\right)
\end{aligned}
$$

Finally, the antisymmetric part of $W_{\mu \nu}^{\mathrm{I} 2}(q)$ vanishes, see eq. (6.65).

$$
W_{\mu \nu}^{\mathrm{ZA}}(q)=-2 i \epsilon_{\mu \nu \lambda \rho} q^{\lambda} \sum_{f} a_{f} c_{f}^{2}\left[\frac{Z^{\rho}}{Q_{\|}} \mathcal{W}_{1}^{\mathrm{F} f}(q)+X^{\rho} \frac{Q_{\perp}}{Q_{\|} Q} \mathcal{W}_{2}^{\mathrm{F} f}(q)\right]
$$

\subsubsection{Angular coefficients}

Rewriting the differential cross section 2.5 in terms of hadronic tensors listed in previous section, we obtain

$$
d \sigma=\frac{d^{4} q}{q^{4}} \frac{e^{4}}{N_{c} s \pi^{2}} \int d^{3} l d^{3} l^{\prime} \delta\left(q-l-l^{\prime}\right) \mathbb{W}\left(q, l, l^{\prime}\right)=\frac{e^{4}}{16 \pi^{2} s N_{c}} \frac{d Q^{2}}{Q^{2}} d Y d^{2} q_{\perp} d \Omega_{l} \mathbb{W}\left(q, l, l^{\prime}\right)
$$

where

$$
\begin{aligned}
\mathbb{W}\left(q, l, l^{\prime}\right)= & \frac{2}{Q^{2}} L^{\mu \nu} \sum_{f} e_{f}^{2}\left[W_{\mu \nu}^{\mathrm{F} f}(q)+W_{\mu \nu}^{\mathrm{H} f}(q)\right] \\
& +\frac{2 c_{e}^{2}}{Q^{2}} \phi_{1}\left(Q^{2}\right)\left\{\left(a_{e}^{2}+1\right) L^{\mu \nu} \sum_{f} c_{f}^{2}\left[\left(a_{f}^{2}+1\right) W_{\mu \nu}^{f \mathrm{~F}}(q)+\left(a_{f}^{2}-1\right) W_{\mu \nu}^{f \mathrm{H}}(q)\right]\right. \\
& \left.-4 a_{e} a_{f} c_{f}^{2} \epsilon^{\mu \nu \alpha \beta} l_{\alpha} l^{\prime} \epsilon_{\mu \nu \lambda \rho} q^{\lambda}\left[\frac{Z^{\rho}}{Q_{\|}} \mathcal{W}_{1}^{\mathrm{F} f}(q)+X^{\rho} \frac{Q_{\perp}}{Q_{\|} Q} \mathcal{W}_{2}^{\mathrm{F} f}(q)\right]\right\} \\
& +\frac{4 c_{e}}{Q^{2}} \phi_{2}\left(Q^{2}\right) \sum_{f} c_{f} e_{f}\left\{a_{e}\left[W_{\mu \nu}^{\mathrm{Ff}}(q)+W_{\mu \nu}^{\mathrm{Hf}}(q)\right] L^{\mu \nu}\right. \\
& \left.-\epsilon^{\mu \nu \alpha \beta} l_{\alpha} l^{\prime} \epsilon_{\mu \nu \lambda \rho} q^{\lambda}\left[\frac{Z^{\rho}}{Q_{\|}} \mathcal{W}_{1}^{\mathrm{F} f}(q)+X^{\rho} \frac{Q_{\perp}}{Q_{\|} Q} \mathcal{W}_{2}^{\mathrm{F} f}(q)\right]\right\} \\
& -\frac{4 c_{e} a_{e}}{Q^{2}} \phi_{3}\left(Q^{2}\right) \sum_{f} c_{f} e_{f}\left[\frac{Q}{Q_{\|}} Y_{\mu} X_{\nu} W_{1}^{\mathrm{If}}(q)+Y_{\mu} Z_{\nu} \frac{Q_{\perp}}{Q_{\|}} W_{2}^{\mathrm{If}}(q)\right] L^{\mu \nu}
\end{aligned}
$$

and

$$
\phi_{1}\left(Q^{2}\right) \equiv \frac{Q^{4}}{\left|m_{Z}^{2}-Q^{2}\right|^{2}+\Gamma_{Z}^{2} m_{Z}^{2}}, \quad \phi_{2}\left(Q^{2}\right)=\frac{\left(Q^{2}-m_{z}^{2}\right)}{Q^{2}} f_{1}\left(Q^{2}\right), \quad \phi_{3}\left(Q^{2}\right)=\frac{\Gamma_{z} m_{z}}{Q^{2}} f_{1}\left(Q^{2}\right)
$$


The angular dependence in the CS frame can be displayed using the convolutions of leptonic and hadronic tensors are presented in section A.5. One obtains

$$
\begin{aligned}
& \mathbb{W}\left(q, l, l^{\prime}\right)=\sum_{f}\left\{\left[e_{f}^{2}+c_{e}^{2}\left(a_{e}^{2}+1\right) c_{f}^{2}\left(a_{f}^{2}+1\right) \phi_{1}+2 c_{e} a_{e} c_{f} a_{f} \phi_{2}\right]\right. \\
& \quad \times\left[\left(W_{T}^{\mathrm{Ff}}+\frac{W_{L}^{\mathrm{Ff}}}{2}\right)\left(1+\cos ^{2} \theta\right)+\frac{W_{L}^{\mathrm{Ff}}}{2}\left(1-3 \cos ^{2} \theta\right)+W_{\Delta}^{\mathrm{Ff}} \sin 2 \theta \cos \phi+W_{\Delta \Delta}^{\mathrm{Ff}} \sin ^{2} \theta \cos 2 \phi\right] \\
& +\left[e_{f}^{2}+c_{e}^{2}\left(a_{e}^{2}+1\right) c_{f}^{2}\left(a_{f}^{2}-1\right) \phi_{1}+2 c_{e} a_{e} c_{f} a_{f} \phi_{2}\right] \\
& \quad \times\left[\left(W_{T}^{\mathrm{Hf}}+\frac{W_{L}^{\mathrm{Hf}}}{2}\right)\left(1+\cos ^{2} \theta\right)+\frac{W_{L}^{\mathrm{Hf}}}{2}\left(1-3 \cos ^{2} \theta\right)+W_{\Delta}^{\mathrm{Hf}} \sin 2 \theta \sin \phi+W_{\Delta \Delta}^{\mathrm{Hf}} \sin ^{2} \theta \cos 2 \phi\right] \\
& \quad+4 c_{e} c_{f}\left[2 a_{e} a_{f} c_{e} c_{f} \phi_{1}+e_{f} \phi_{2}\right]\left[\mathcal{W}_{1}^{\mathrm{Ff}} \cos \theta+\frac{Q_{\perp}}{Q} \mathcal{W}_{2}^{\mathrm{F} f} \sin \theta \cos \phi\right] \\
& \left.\quad+a_{e} a_{f} c_{e} c_{f} \phi_{3}\left[W_{1}^{\mathrm{If}} \sin ^{2} \theta \sin 2 \phi+\frac{Q_{\perp}}{Q} W_{2}^{\mathrm{If}} \sin 2 \theta \sin \phi\right]\right\}
\end{aligned}
$$

where $\phi_{i}=\phi_{i}\left(Q^{2}\right)$ and $W^{i}=W^{i}\left(\alpha_{q}, \beta_{q}, Q^{2}\right)$.

The above formula (7.18) is the main result of the paper. It should be compared to standard representation of angular distribution of DY leptons (1.3)

\subsection{Comparison with LHC measurements}

The LHC measurements are integrated over the region of invariant mass of DY pair between 80 and $100 \mathrm{GeV}$. In this kinematic region the most important contribution comes from terms in eq. (7.18) multiplied by $\phi_{1}$. Indeed, for an estimate we can consider $W^{i}\left(\alpha_{q}, \beta_{q}, Q^{2}\right) \simeq W^{i}\left(\alpha_{q}, \beta_{q}, m_{z}^{2}\right)$ and get

$$
\int_{80}^{100} \frac{d Q^{2}}{Q^{2}} \simeq 0.2, \quad \int_{80}^{100} \frac{d Q^{2}}{Q^{2}} \phi_{1}\left(Q^{2}\right) \simeq 95, \quad \int_{80}^{100} \frac{d Q^{2}}{Q^{2}} \phi_{2}\left(Q^{2}\right) \simeq 0, \quad \int_{80}^{100} \frac{d Q^{2}}{Q^{2}} \phi_{3}\left(Q^{2}\right) \simeq 2.6
$$

Since the accuracy of our small-x approximation is $\alpha_{q}=x_{A}, \beta_{q}=x_{B} \sim 0.1$ we can neglect all contributions coming form terms not multiplied by $\phi_{1}$. Moreover, both theoretical [28] and phenomenological $[29,30]$ analysis indicate that $h_{1}^{\perp}$ is of order of few percent of $f_{1}$ and hence in numerical estimates we will disregard the contribution of $h_{1}^{\perp}$. Introducing notations

$$
\begin{array}{ll}
\mathcal{W}^{\mathrm{F} f}(q)=\int d^{2} k_{\perp} F^{f}\left(q, k_{\perp}\right), & \mathcal{W}_{L}^{\mathrm{F} f}(q)=\int d k_{\perp} \frac{(q-2 k)_{\perp}^{2}}{q_{\perp}^{2}} F^{f}\left(q, k_{\perp}\right) \\
\mathcal{W}_{1}^{\mathrm{F} f}(q)=\int d^{2} k_{\perp} \frac{(q, q-2 k)_{\perp}}{q_{\perp}^{2}} F^{f}\left(q, k_{\perp}\right) &
\end{array}
$$


we get

$$
\begin{aligned}
\mathbb{W}\left(q, l, l^{\prime}\right)= & c_{e}^{2} c_{f}^{2} \phi_{1}\left(Q^{2}\right) \sum_{f}\left\{( a _ { e } ^ { 2 } + 1 ) ( a _ { f } ^ { 2 } + 1 ) \left(\left[\mathcal{W}^{\mathrm{Ff}}-\frac{Q_{\perp}^{2}}{2 Q^{2}}\left(\mathcal{W}^{\mathrm{Ff}}-\mathcal{W}_{L}^{\mathrm{Ff}}\right)\right]\left(1+\cos ^{2} \theta\right)\right.\right. \\
& \left.\left.+\frac{Q_{\perp}^{2}}{2 Q^{2}} \mathcal{W}_{L}^{\mathrm{Ff}}\left(1-3 \cos ^{2} \theta\right)+\frac{Q_{\perp}}{Q} \mathcal{W}_{1}^{\mathrm{Ff}} \sin 2 \theta \cos \phi+\frac{Q_{\perp}^{2}}{2 Q^{2}} \mathcal{W}^{\mathrm{Ff}} \sin ^{2} \theta \cos 2 \phi\right]\right) \\
& \left.+8 a_{e} a_{f}\left[\frac{Q_{\perp}}{Q} \mathcal{W}_{3}^{\mathrm{F} f} \sin \theta \cos \phi+\mathcal{W}_{4}^{\mathrm{Ff}} \cos \theta\right]\right\}
\end{aligned}
$$

where $\mathcal{W}_{3}^{\mathrm{F} f}$ and $\mathcal{W}_{4}^{\mathrm{F} f}$ are defined in eq. (7.9). Since we neglected exchange-type power corrections we should expect the accuracy of order of $\frac{1}{N_{c}} \sim 30 \%$. Let us discuss now some qualitative and semi-quantitative predictions of this equation. First, let us evaluate $\mathcal{W}_{i}$ at $Q_{\perp}^{2} \gg m^{2}$ following ref. [14].

\subsubsection{Logarithmical estimates of $\mathcal{W}_{i}$ at $Q_{\perp}^{2} \gg m^{2}$}

At $q_{\perp}^{2} \gg m^{2}$ we probe the perturbative tail of TMD $f_{1}$ which is $\sim \frac{1}{k_{\perp}^{2}}$ So, as long as $Q^{2} \gg q_{\perp}^{2} \gg m^{2}$ we can approximate

$$
f_{1}\left(\alpha_{z}, k_{\perp}^{2}\right) \simeq \frac{f\left(\alpha_{q}\right)}{k_{\perp}^{2}}, \quad \bar{f}_{1} \simeq \frac{\bar{f}\left(\alpha_{q}\right)}{k_{\perp}^{2}}
$$

(up to logarithmic corrections). Similarly, for the target we can use the estimate

$$
f_{1}\left(\beta_{z}, k_{\perp}^{2}\right) \simeq \frac{f\left(\beta_{z}\right)}{k_{\perp}^{2}}, \quad \bar{f}_{1} \simeq \frac{\bar{f}\left(\beta_{z}\right)}{k_{\perp}^{2}}
$$

as long as $k_{\perp}^{2} \ll Q^{2}$. Thus, we get an estimate

$$
\begin{array}{ll}
F^{f}\left(q, k_{\perp}\right) \simeq \frac{F^{f}\left(\alpha_{q}, \beta_{q}\right)}{k_{\perp}^{2}(q-k)_{\perp}^{2}}, & F^{f}\left(\alpha_{q}, \beta_{q}\right) \equiv f^{f}\left(\alpha_{q}\right) \bar{f}^{f}\left(\beta_{q}\right)+f^{f} \leftrightarrow \bar{f}^{f}, \\
\mathcal{F}^{f}\left(q, k_{\perp}\right) \simeq \frac{\mathcal{F}^{f}\left(\alpha_{q}, \beta_{q}\right)}{k_{\perp}^{2}(q-k)_{\perp}^{2}}, & \mathcal{F}^{f}\left(\alpha_{q}, \beta_{q}\right) \equiv f^{f}\left(\alpha_{q}\right) \bar{f}^{f}\left(\beta_{q}\right)-f^{f} \leftrightarrow \bar{f}^{f} .
\end{array}
$$

Due to eqs. (7.22) and (7.23), the integrals over $k_{\perp}$ are logarithmic and should be cut from below by $m_{N}^{2}$ and from above by $Q^{2}$ so we get an estimate

$$
\int d^{2} k_{\perp} \frac{1}{k_{\perp}^{2}(q-k)_{\perp}^{2}} \simeq \frac{2 \pi}{q_{\perp}^{2}} \ln \frac{q_{\perp}^{2}}{m^{2}}, \quad \int d^{2} k_{\perp} \frac{(k, q-k)_{\perp}}{k_{\perp}^{2}(q-k)_{\perp}^{2}} \simeq-\pi \ln \frac{Q^{2}}{q_{\perp}^{2}}
$$

where we assumed that the first integral is determined by the logarithmical region $q_{\perp}^{2} \gg$ $k_{\perp}^{2} \gg m_{N}^{2}$ and the second by $Q^{2} \gg k_{\perp}^{2} \gg q_{\perp}^{2}$. Taking these integrals to eq. (7.20) one obtains

$$
\begin{array}{ll}
\mathcal{W}^{\mathrm{F} f}(q) \simeq \frac{2 \pi}{Q_{\perp}^{2}} \ln \frac{q_{\perp}^{2}}{m^{2}} F^{f}\left(\alpha_{q}, \beta_{q}\right), & \mathcal{W}_{L}^{\mathrm{F} f}(q) \simeq \frac{2 \pi}{Q_{\perp}^{2}}\left[\ln \frac{Q_{\perp}^{2}}{m^{2}}+2 \ln \frac{Q^{2}}{Q_{\perp}^{2}}\right] F^{f}\left(\alpha_{q}, \beta_{q}\right) \\
\mathcal{W}_{4}^{\mathrm{F} f}(q) \simeq \frac{2 \pi}{Q_{\perp}^{2}} \ln \frac{q_{\perp}^{2}}{m^{2}} \mathcal{F}^{f}\left(\alpha_{q}, \beta_{q}\right), & \mathcal{W}_{1}^{\mathrm{F} f}(q) \simeq \mathcal{W}_{3}^{\mathrm{F} f}(q) \simeq 0 .
\end{array}
$$




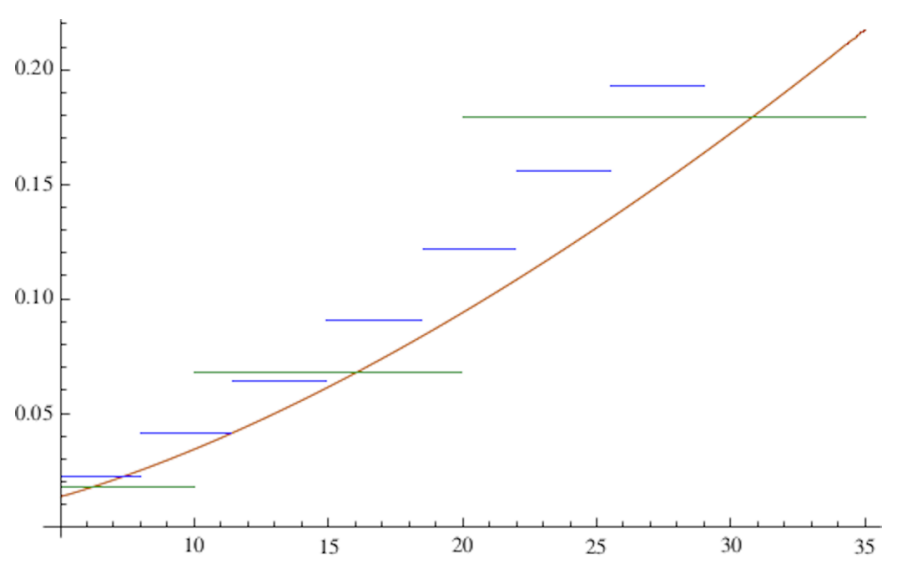

Figure 4. Comparison of prediction (7.29) with lines depicting angular coefficient $A_{0}$ in bins of $Q_{\perp}$ and $Y<1$ from ref. [18]. (long bins) and ref. [17] (short bins).

For numerical estimates of $A_{0}$ and $A_{2}$ we will need the ratio

$$
r\left(Q_{\perp}^{2}\right) \equiv \frac{\mathcal{W}_{L}^{\mathrm{Ff}}(q)}{\mathcal{W}^{\mathrm{Ff}}(q)} \simeq 1+2 \frac{\ln Q^{2} / Q_{\perp}^{2}}{\ln Q_{\perp}^{2} / m^{2}}
$$

which does not depend on quark flavors. Substituting these formulas to eq. (7.18), we get we get

$$
\begin{aligned}
\mathbb{W}\left(q, l, l^{\prime}\right)= & c_{e}^{2}\left(a_{e}^{2}+1\right) c_{f}^{2} \phi_{1}\left(Q^{2}\right) \sum_{f}\left(a_{f}^{2}+1\right) \mathcal{W}^{\mathrm{Ff}}\left\{\left(\left[1-\frac{Q_{\perp}^{2}}{2 Q^{2}}(1-r)\right]\left(1+\cos ^{2} \theta\right)\right.\right. \\
& \left.\left.\left.+\frac{Q_{\perp}^{2}}{2 Q^{2}} r\left(1-3 \cos ^{2} \theta\right)+\frac{Q_{\perp}^{2}}{2 Q^{2}} \sin ^{2} \theta \cos 2 \phi\right]\right)+\frac{8 a_{e} a_{f}}{\left(a_{e}^{2}+1\right)\left(a_{f}^{2}+1\right)} \frac{\mathcal{F}^{f}}{F^{f}} \cos \theta\right\}
\end{aligned}
$$

\subsubsection{Estimates of $A_{0}$ and $A_{2}$}

Since our estimate (7.27) does not depend on flavor, from eq. (7.28) we get

$$
A_{0}=\frac{Q_{\perp}^{2}}{m_{z}^{2}} \frac{1+2 \frac{\ln m_{z}^{2} / Q_{\perp}^{2}}{\ln Q_{\perp}^{2} / m^{2}}}{1+\frac{Q_{\perp}^{2}}{m_{z}^{2}} \frac{\ln m_{z}^{2} / Q_{\perp}^{2}}{\ln Q_{\perp}^{2} / m^{2}}}
$$

where we replaced $Q^{2}$ by $m_{Z}^{2}$ since this function varies slowly between 80 and $100 \mathrm{GeV}$. This formula is compared to LHC measurements in figure 4 . The accuracy of our estimate is about $20 \%$ which is reasonable since we neglected corrections $\sim \frac{1}{N_{c}}$.

Next, in our logarithmic approximation

$$
A_{2}=\frac{Q_{\perp}^{2} / m_{z}^{2}}{1+\frac{Q_{\perp}^{2}}{m_{z}^{2}} \frac{\ln m_{z}^{2} / Q_{\perp}^{2}}{\ln Q_{\perp}^{2} / m^{2}}}
$$

The comparison with LHC data is shown in figure 5. The accuracy here is about $30 \%$. 


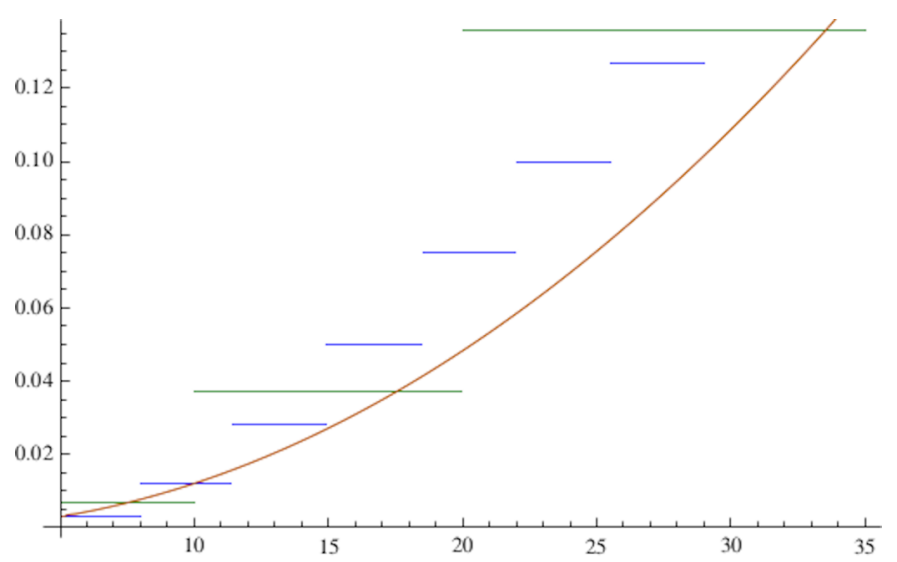

Figure 5. Comparison of prediction (7.30) with lines depicting angular coefficient $A_{2}$ in bins of $Q_{\perp}$ and $Y<1$ from ref. [18]. (long bins) and [17] (short bins).

\subsubsection{Qualitative checks for other angular coefficients}

First, note that between 10 and $30 \mathrm{GeV}$ the coefficient $A_{1}$ from table 15 of ref. [17] is an order of magnitude smaller than $A_{0}$ from table 14 (or $A_{2}$ from table 16) in accordance with our estimate $A_{1} \simeq 0$. This is another argument in favor of factorization hypothesis $f_{1}\left(x_{B}, k_{\perp}\right) \simeq f_{1}\left(x_{B}\right) g\left(k_{\perp}\right)$ which is frequently used in current TMD literature.

Second, from eq. (7.28) we see that our estimate for $A_{4}$ involves non-trivial flavor structure, but one thing immediately seen is that $A_{4}$ does not depend on $Q_{\perp}$ in our region $10-30 \mathrm{GeV}$. As one can see from table 18 of ref. [17], the experimental numbers for $A_{4}$ almost do not change in this kinematical region. Moreover, from eq. (7.24) $\mathcal{F}^{f}\left(\alpha_{q}, \beta_{q}\right)=0$ at $Y=0$ (i.e., when $\alpha_{q}=\beta_{q}$ ) so we should expect that $A_{4}$ in rapidity bin $2>Y>1$ is greater than in bin $Y<1$, and table 18 confirms that. On the contrary, the results for $A_{0}$ and $A_{2}$ do not change much between those rapidity beans in accordance with our prediction that $F^{f}\left(\alpha_{q}, \beta_{q}\right)$ does not change radically.

Third, our estimate $A_{3}=0$ is in accordance to the fact that experimental numbers for $A_{3}$ from table 17 of ref. [17] are order of magnitude smaller than numbers $A_{4}$ from table 18.

Finally, the coefficients $A_{5}$ to $A_{7}$ do not appear in our estimate (7.18), again in accordance with tables 19-21 of ref. [17] where the numbers for $A_{5}, A_{6}$, and $A_{7}$ are actually two orders of magnitude smaller than the numbers for $A_{0}$ or $A_{2}$ in our kinematical region.

Summarizing, it looks like our $f_{1}$-based estimates point in the right direction, but of course more phenomenological work is required. Note also that in ref. [16] it was demonstrated that TMD evolution in the double-log approximation does not affect the predictions for angular coefficients, but the single-log corrections to TMD evolution may change the results for asymmetries. The study is in progress.

\section{Conclusions and outlook}

To my knowledge, this is the first analysis of DY angular coefficients in the framework of TMD factorization, and, as we saw, the first signs are encouraging. There are two questions 
about the TMD analysis of DY process: can we predict/explain angular dependence of DY cross section from TMD factorization at LHC kinematical range and reciprocally, can we learn something about proton structure from this?

The answer to first question is probably yes. We see that when we have sufficient knowledge about TMDs responsible for a particular angular coefficient, that coefficient comes out with reasonable accuracy even with back-of-the-envelope estimates. For example, the very naive estimate of the coefficients $A_{0}$ and $A_{2}$ agrees with experiment at $30 \%$ accuracy. The error may be due to our approximation of TMDs by perturbative tails or maybe it is due to $\frac{1}{N_{c}}$ corrections proportional to higher-twist operators. I hope that careful analysis involving established models of $f_{1}\left(x, q_{\perp}\right)$ will distinguish between these two possibilities. As to the rest of angular coefficients, more details about quark TMDs and at least some guesses about quark-quark-gluon TMDs are necessary to make quantitative predictions. Also, one needs to take into account fiducial power corrections, see recent paper [19] for a review.

This bring us to the second question, namely how much we can learn about proton structure from $Z$-boson experiments. First, as was demonstrated above, the coefficients $A_{0}$ and $A_{2}$ are determined by TMD $f_{1}$ with $\frac{1}{N_{c}} \sim 30 \%$ accuracy. We used the naive logarithmic estimates and it is natural to assume that the realistic models for $f_{1}$ will move the curves in figures 4 and 5 closer to the experimental points. Also, it should be mentioned, tat the "factorization hypothesis" for the LT TMDs like $f\left(x_{B}, k_{\perp}\right) \simeq f\left(x_{B}\right) g\left(k_{\perp}\right)$ seems to be confirmed by experimental data in a sense that the angular coefficients which vanish in this approximation are smaller than the non-vanishing ones. The coefficients $A_{1}, A_{3}$, and $A_{4}$ are determined by non-factorized part of $f_{1}$ or higher-twist exchange contributions.

Next, the coefficients $A_{5}-A_{7}$ seem to be determined by exchange-type corrections proportional to higher-twist quark-quark-gluon TMDs. Unfortunately, comprehensive analysis of exchange-type power corrections requires calculation of higher-twist contributions restoring EM gauge invariance of these corrections, see the discussion in section 4.5.

Let us also discuss the perturbative corrections to asymmetries. As demonstrated in ref. [16] our estimates of asymmetries are not affected by summation of Sudakov double logs, but single logs may bring some changes to tree-level results. It should be also emphasized that, as discussed in refs. [14, 15], from the rapidity factorization (3.2) we get TMDs with rapidity-only cutoff $|\alpha|<\sigma_{t}$ or $|\beta|<\sigma_{p}$. Such cutoff, relevant for small- $x$ physics, is different from the combination of UV and rapidity cutoffs for TMDs used by moderate- $x$ community, see the analysis in two [31-33] and three [34] loops. This difference in cutoffs does not matter for the tree-level formulas of section 7 , but if one goes beyond the tree level, one has to relate TMDs with rapidity-only cutoffs to the TMD models with conventional cutoffs. This requires calculations at the NLO level which are in progress.

The author is grateful to A. Prokudin and A. Vladimirov for valuable discussions. This work is supported by Jefferson Science Associates, LLC under the U.S. DOE contract \#DE-AC05-06OR23177 and by U.S. DOE grant \#DE-FG02-97ER41028. 


\section{A Frequently used formulas}

\section{A.1 Formulas with Dirac matrices}

\section{A.1.1 Fierz transformations}

First, let us write down Fierz transformations for symmetric and antisymmetric combinations

$$
\begin{aligned}
& \frac{1}{2}\left[\left(\bar{\psi} \gamma_{\mu} \chi\right)\left(\bar{\chi} \gamma_{\nu} \psi\right)+\mu \leftrightarrow \nu\right] \\
& =-\frac{1}{4}\left(\delta_{\mu}^{\alpha} \delta_{\nu}^{\beta}+\delta_{\nu}^{\alpha} \delta_{\mu}^{\beta}-g_{\mu \nu} g^{\alpha \beta}\right)\left[\left(\bar{\psi} \gamma_{\alpha} \psi\right)\left(\bar{\chi} \gamma_{\beta} \chi\right)+\left(\bar{\psi} \gamma_{\alpha} \gamma_{5} \psi\right)\left(\bar{\chi} \gamma_{\beta} \gamma_{5} \chi\right)\right] \\
& \quad+\frac{1}{4}\left(\delta_{\mu}^{\alpha} \delta_{\nu}^{\beta}+\delta_{\nu}^{\alpha} \delta_{\mu}^{\beta}-\frac{1}{2} g_{\mu \nu} g^{\alpha \beta}\right)\left(\bar{\psi} \sigma_{\alpha \xi} \psi\right)\left(\bar{\chi} \sigma_{\beta}^{\xi} \chi\right)-\frac{g_{\mu \nu}}{4}(\bar{\psi} \psi)(\bar{\chi} \chi)+\frac{g_{\mu \nu}}{4}\left(\bar{\psi} \gamma_{5} \psi\right)\left(\bar{\chi} \gamma_{5} \chi\right)
\end{aligned}
$$

and

$$
\begin{aligned}
& \frac{1}{2}\left[\left(\bar{\psi} \gamma_{\mu} \chi\right)\left(\bar{\chi} \gamma_{\nu} \psi\right)-\mu \leftrightarrow \nu\right]=\frac{i}{4} \epsilon_{\mu \nu \alpha \beta}\left[\left(\bar{\psi} \gamma_{\alpha} \gamma_{5} \psi\right)\left(\bar{\chi} \gamma_{\beta} \chi\right)+\left(\bar{\psi} \gamma_{\alpha} \psi\right)\left(\bar{\chi} \gamma_{\beta} \gamma_{5} \chi\right)\right] \\
& -\frac{i}{4}(\bar{\psi} \psi)\left(\bar{\chi} \sigma_{\mu \nu} \chi\right)+\frac{i}{4}\left(\bar{\psi} \gamma_{5} \psi\right)\left(\bar{\chi} \sigma_{\mu \nu} \gamma_{5} \chi\right)+\frac{i}{4}\left(\bar{\psi} \sigma_{\mu \nu} \psi\right)(\bar{\chi} \chi)-\frac{i}{4}\left(\bar{\psi} \sigma_{\mu \nu} \gamma_{5} \psi\right)\left(\bar{\chi} \gamma_{5} \chi\right)
\end{aligned}
$$

Using eq. (A.11) one can obtain the following formula

$$
\begin{aligned}
& \frac{1}{4}\left[\left(\bar{\psi} \gamma_{\mu} \chi\right)\left(\bar{\chi} \gamma_{\nu} \gamma_{5} \psi\right)+\left(\bar{\psi} \gamma_{\mu} \gamma_{5} \chi\right)\left(\bar{\chi} \gamma_{\nu} \psi\right)+\mu \leftrightarrow \nu\right] \\
& =-\frac{1}{4}\left(\delta_{\mu}^{\alpha} \delta_{\nu}^{\beta}+\delta_{\nu}^{\alpha} \delta_{\mu}^{\beta}-g_{\mu \nu} g^{\alpha \beta}\right)\left[\left(\bar{\psi} \gamma_{\alpha} \gamma_{5} \psi\right)\left(\bar{\chi} \gamma_{\beta} \chi\right)+\left(\bar{\psi} \gamma_{\alpha} \psi\right)\left(\bar{\chi} \gamma_{\beta} \gamma_{5} \chi\right)\right]
\end{aligned}
$$

We need also

$$
\begin{aligned}
& \frac{1}{4}\left[\left(\bar{\psi} \gamma_{\mu} \chi\right)\left(\bar{\chi} \gamma_{\nu} \gamma_{5} \psi\right)-\left(\bar{\psi} \gamma_{\mu} \gamma_{5} \chi\right)\left(\bar{\chi} \gamma_{\nu} \psi\right)+\mu \leftrightarrow \nu\right]=\frac{g_{\mu \nu}}{4}(\bar{\psi} \psi)\left(\bar{\chi} \gamma_{5} \chi\right) \\
& -\frac{g_{\mu \nu}}{4}\left(\bar{\psi} \gamma_{5} \psi\right)(\bar{\chi} \chi)-\frac{1}{8}\left[\left(\bar{\psi} \sigma_{\mu \xi} \psi\right)\left(\bar{\chi} \sigma_{\nu}{ }^{\xi} \gamma_{5} \chi\right)-\left(\bar{\psi} \sigma_{\mu \xi} \gamma_{5} \psi\right)\left(\bar{\chi} \sigma_{\nu}^{\xi} \chi\right)+\mu \leftrightarrow \nu\right]
\end{aligned}
$$

for symmetric tensors and

$$
\begin{aligned}
& \frac{1}{4}\left[\left(\bar{\psi} \gamma_{\mu} \chi\right)\left(\bar{\chi} \gamma_{\nu} \gamma_{5} \psi\right)+\left(\bar{\psi} \gamma_{\mu} \gamma_{5} \chi\right)\left(\bar{\chi} \gamma_{\nu} \psi\right)-\mu \leftrightarrow \nu\right] \\
& =\frac{i}{4} \epsilon_{\mu \nu \alpha \beta}\left[\left(\bar{\psi} \gamma_{\alpha} \psi\right)\left(\bar{\chi} \gamma_{\beta} \chi\right)+\left(\bar{\psi} \gamma_{\alpha} \gamma_{5} \psi\right)\left(\bar{\chi} \gamma_{\beta} \gamma_{5} \chi\right)\right] \\
& \frac{1}{4}\left[\left(\bar{\psi} \gamma_{\mu} \chi\right)\left(\bar{\chi} \gamma_{\nu} \gamma_{5} \psi\right)-\left(\bar{\psi} \gamma_{\mu} \gamma_{5} \chi\right)\left(\bar{\chi} \gamma_{\nu} \psi\right)-\mu \leftrightarrow \nu\right] \\
& =-\frac{i}{4}\left(\bar{\psi} \gamma_{5} \psi\right)\left(\bar{\chi} \sigma_{\mu \nu} \chi\right)+\frac{i}{4}(\bar{\psi} \psi)\left(\bar{\chi} \sigma_{\mu \nu} \gamma_{5} \chi\right)+\frac{i}{4}\left(\bar{\psi} \sigma_{\mu \nu} \gamma_{5} \psi\right)(\bar{\chi} \chi)-\frac{i}{4}\left(\bar{\psi} \sigma_{\mu \nu} \psi\right)\left(\bar{\chi} \gamma_{5} \chi\right)
\end{aligned}
$$

for the antisymmetric ones. 


\section{A.1.2 Formulas with $\sigma$-matrices}

It is convenient to define ${ }^{10}$

$$
\epsilon_{i j} \equiv \frac{2}{s} \epsilon_{\star \bullet i j}=\frac{2}{s} p_{2}^{\mu} p_{1}^{\nu} \epsilon_{\mu \nu i j}
$$

such that $\epsilon_{12}=1$ and $\epsilon_{i j} \epsilon_{k l}=g_{i k} g_{j l}-g_{i l} g_{j k}$. The frequently used formula is

$$
\sigma_{\mu \nu} \sigma_{\alpha \beta}=\left(g_{\mu \alpha} g_{\nu \beta}-g_{\mu \beta} g_{\nu \alpha}\right)-i \epsilon_{\mu \nu \alpha \beta} \gamma_{5}-i\left(g_{\mu \alpha} \sigma_{\nu \beta}-g_{\mu \beta} \sigma_{\nu \alpha}-g_{\nu \alpha} \sigma_{\mu \beta}+g_{\nu \beta} \sigma_{\mu \alpha}\right)
$$

with variations

$$
\begin{aligned}
\frac{2}{s} \sigma_{\bullet i} \sigma_{\star j} & =g_{i j}-i \epsilon_{i j} \gamma_{5}-i \sigma_{i j}-\frac{2 i}{s} g_{i j} \sigma_{\bullet \star}, & \frac{2}{s} \sigma_{\star i} \sigma_{\bullet j} & =g_{i j}+i \epsilon_{i j} \gamma_{5}-i \sigma_{i j}+\frac{2}{s} g_{i j} \sigma_{\star \bullet}, \\
\sigma_{i j} \sigma_{\bullet k} & =-\sigma_{\bullet k} \sigma_{i j}=-i g_{i k} \sigma_{\bullet j}+i g_{j k} \sigma_{\bullet}, & \sigma_{i j} \sigma_{\star k} & =-\sigma_{\star k} \sigma_{i j}=-i g_{i k} \sigma_{\star j}+i g_{j k} \sigma_{\star i}
\end{aligned}
$$

We need also the following formulas with $\sigma$-matrices in different matrix elements

$$
\begin{aligned}
& \tilde{\sigma}_{\mu \nu} \otimes \tilde{\sigma}_{\alpha \beta}=-\frac{1}{2}\left(g_{\mu \alpha} g_{\nu \beta}-g_{\nu \alpha} g_{\mu \beta}\right) \sigma_{\xi \eta} \otimes \sigma^{\xi \eta} \\
& \quad+g_{\mu \alpha} \sigma_{\beta \xi} \otimes \sigma_{\nu}^{\xi}-g_{\nu \alpha} \sigma_{\beta \xi} \otimes \sigma_{\mu}^{\xi}-g_{\mu \beta} \sigma_{\alpha \xi} \otimes \sigma_{\nu}^{\xi}+g_{\nu \beta} \sigma_{\alpha \xi} \otimes \sigma_{\mu}^{\xi}-\sigma_{\alpha \beta} \otimes \sigma_{\mu \nu}
\end{aligned}
$$

and

$$
\begin{gathered}
\tilde{\sigma}_{\mu \xi} \otimes \tilde{\sigma}_{\nu}^{\xi}=-\frac{g_{\mu \nu}}{2} \sigma_{\xi \eta} \otimes \sigma^{\xi \eta}+\sigma_{\nu \xi} \otimes \sigma_{\mu}^{\xi}, \quad \sigma_{\xi \eta} \otimes \tilde{\sigma}^{\xi \eta}=\tilde{\sigma}_{\xi \eta} \otimes \sigma^{\xi \eta} \\
\sigma_{\mu \xi} \gamma_{5} \otimes \sigma_{\nu}{ }^{\xi} \gamma_{5}+\mu \leftrightarrow \nu-\frac{g_{\mu \nu}}{2} \sigma_{\xi \eta} \gamma_{5} \otimes \sigma^{\xi \eta} \gamma_{5}=-\left[\sigma_{\mu \xi} \otimes \sigma_{\nu}{ }^{\xi}+\mu \leftrightarrow \nu-\frac{g_{\mu \nu}}{2} \sigma_{\xi \eta} \otimes \sigma^{\xi \eta}\right] \\
\tilde{\sigma}_{\star j} \otimes \tilde{\sigma}_{\bullet k}=-\frac{s}{4} g_{j k} \sigma_{\xi \eta} \otimes \sigma^{\xi \eta}+\frac{s}{2} \sigma_{k \xi} \otimes \sigma_{j}{ }^{\xi}+g_{j k} \sigma_{\bullet \xi} \otimes \sigma_{\star}^{\xi}-\sigma_{\bullet k} \otimes \sigma_{\star j} \\
=-\frac{s}{4} g_{j k} \sigma_{m n} \otimes \sigma^{m n}-g_{j k} \sigma_{\star l} \otimes \sigma_{\bullet}^{l}+\sigma_{\star k} \otimes \sigma_{\bullet j}+\frac{s}{2} \sigma_{k l} \otimes \sigma_{j}^{l} \\
\sigma_{\star i} \otimes \sigma_{\bullet j}-\sigma_{\star i} \gamma_{5} \otimes \sigma_{\bullet j} \gamma_{5} \\
=-g_{i j}^{\perp} \sigma_{\star l} \otimes \sigma_{\bullet}{ }^{l}+\sigma_{\star j} \otimes \sigma_{\bullet i}+\sigma_{\star i} \otimes \sigma_{\bullet j}-\frac{s}{4} g_{i j}^{\perp} \sigma_{m n} \otimes \sigma^{m n}+\frac{s}{2} \sigma_{j l} \otimes \sigma_{i}{ }^{l} \\
\sigma_{\star}^{k} \otimes \gamma_{i} \sigma_{\bullet k} \gamma_{j}=\hat{p}_{2} \gamma^{k} \otimes \not p_{1} \gamma_{i} \gamma_{k} \gamma_{j}=\hat{p}_{2} \gamma^{k} \otimes \not p_{1}\left(g_{i k} \gamma_{j}+g_{j k} \gamma_{i}-g_{i j} \gamma_{k}\right) \\
=\hat{p}_{2}\left(g_{i k} \gamma_{j}+g_{j k} \gamma_{i}-g_{i j} \gamma_{k}\right) \otimes \not p_{1} \gamma^{k}=\left(\gamma_{j} \sigma_{\star}{ }^{k} \gamma_{i}\right) \otimes \sigma_{\bullet k}
\end{gathered}
$$

We will need also

$$
\not p_{2} \otimes \gamma_{i} \not p_{1} \gamma_{j}+\not p_{2} \gamma_{5} \otimes \gamma_{i} \not p_{1} \gamma_{j} \gamma_{5}=\gamma_{j} \not p_{2} \gamma_{i} \otimes \not p_{1}+\gamma_{j} \not p_{2} \gamma_{i} \gamma_{5} \otimes \not p_{1} \gamma_{5}
$$

\footnotetext{
${ }^{10}$ We use conventions from Bjorken $\varepsilon^{3}$ D rell where $\epsilon^{0123}=-1$ and $\gamma^{\mu} \gamma^{\nu} \gamma^{\lambda}=g^{\mu \nu} \gamma^{\lambda}+g^{\nu \lambda} \gamma^{\mu}-g^{\mu \lambda} \gamma^{\nu}-$ $i \epsilon^{\mu \nu \lambda \rho} \gamma_{\rho} \gamma_{5}$. Also, with this convention $\tilde{\sigma}_{\mu \nu} \equiv \frac{1}{2} \epsilon_{\mu \nu \lambda \rho} \sigma^{\lambda \rho}=i \sigma_{\mu \nu} \gamma_{5}$.
} 


\section{A.1.3 Formulas with $\gamma$-matrices and one gluon field}

In the gauge $A_{\bullet}=0$ the "projectile" field $A_{i}$ can be represented as

$$
A_{i}\left(x_{\bullet}, x_{\perp}\right)=\frac{2}{s} \int_{-\infty}^{x \bullet} d x_{\bullet}^{\prime} F_{\star i}^{(A)}\left(x_{\bullet}^{\prime}, x_{\perp}\right)
$$

and similarly for the "target" field

$$
B_{i}\left(x_{\bullet}, x_{\perp}\right)=\frac{2}{s} \int_{-\infty}^{x_{\star}} d x_{\star}^{\prime} F_{\star i}^{(B)}\left(x_{\star}^{\prime}, x_{\perp}\right)
$$

in the $B_{\bullet}=0$ gauge. It is convenient to define

$$
\tilde{A}_{i}\left(x_{\bullet}, x_{\perp}\right)=\frac{2}{s} \int_{-\infty}^{x \bullet} d x_{\bullet}^{\prime} \tilde{F}_{\star i}^{(A)}\left(x_{\bullet}^{\prime}, x_{\perp}\right), \quad \tilde{B}_{i}\left(x_{\star}, x_{\perp}\right)=\frac{2}{s} \int_{-\infty}^{x_{\star}} d x_{\star}^{\prime} \tilde{F}_{\bullet i}^{(B)}\left(x_{\star}^{\prime}, x_{\perp}\right),
$$

where $\tilde{F}_{\mu \nu}=\frac{1}{2} \epsilon_{\mu \nu \lambda \rho} F^{\lambda \rho}$ as usual. With this definition we have $\tilde{A}_{i}=-\epsilon_{i j} A^{j}$ and $\tilde{B}_{i}=\epsilon_{i j} B^{j}$ so

$$
\not p_{2} \breve{A}_{i}=-\not A \not p_{2} \gamma_{i}, \quad \breve{A}_{i} \not p_{2}=-\gamma_{i} \not \not_{2} \not A, \quad \not \breve{B}_{1} \breve{B}_{i}=-\not B \not p_{1} \gamma_{i}, \quad \breve{B}_{i} \not p_{1}=-\gamma_{i} \not \not_{1} \not D
$$

We also used

$$
\begin{aligned}
& A^{i} \not p_{2} \otimes \gamma_{n} \not p_{1} \gamma_{i}+A^{i} \not p_{2} \gamma_{5} \otimes \gamma_{n} \not p_{1} \gamma_{i} \gamma_{5}=-\not p_{2} \breve{A}_{n} \otimes \not p_{1}-\not p_{2} \breve{A}_{n} \gamma_{5} \otimes \not p_{1} \gamma_{5} \\
& A^{i} \not p_{2} \otimes \gamma_{i} \not p_{1} \gamma_{n}+A^{i} \not p_{2} \gamma_{5} \otimes \gamma_{i} \not p_{1} \gamma_{n} \gamma_{5}=-\breve{A}_{n} \not p_{2} \otimes \not p_{1}-\breve{A}_{n} \not p_{2} \gamma_{5} \otimes \not p_{1} \gamma_{5} \\
& \gamma_{n} \not p_{2} \gamma^{i} \otimes \not p_{1} B_{i}+\gamma_{n} \not p_{2} \gamma^{i} \gamma_{5} \otimes \not p_{1} \gamma_{5} B_{i}=-\not p_{2} \otimes \not p_{1} \breve{B}_{n}-\not p_{2} \gamma_{5} \otimes \not p_{1} \breve{B}_{n} \gamma_{5} \\
& \gamma^{i} \not p_{2} \gamma_{n} \otimes \not p_{1} B_{i}+\gamma^{i} \not p_{2} \gamma_{n} \gamma_{5} \otimes \not p_{1} \gamma_{5} B_{i}=-\not p_{2} \otimes \breve{B}_{n} \not p_{1}-\not p_{2} \gamma_{5} \otimes \breve{B}_{n} \not p_{1} \gamma_{5}
\end{aligned}
$$

and

$$
\begin{aligned}
& \frac{2}{s}\left[\not p_{1} \not p_{2} \gamma_{i} \otimes B^{i} \gamma_{n}+\not p_{1} \not \not_{2} \gamma_{i} \gamma_{5} \otimes B^{i} \gamma_{n} \gamma_{5}\right]=\gamma_{i} \otimes \gamma_{n} \breve{B}_{i}+\gamma_{i} \gamma_{5} \otimes \gamma_{n} \breve{B}_{i} \gamma_{5} \\
& \frac{2}{s}\left[\gamma_{i} \not p_{2} \not p_{1} \otimes B^{i} \gamma_{n}+\gamma_{i} \not p_{2} \not p_{1} \gamma_{5} \otimes B^{i} \gamma_{n} \gamma_{5}\right]=\gamma_{i} \otimes \breve{B}_{i} \gamma_{n}+\gamma_{i} \gamma_{5} \otimes \breve{B}_{i} \gamma_{n} \gamma_{5} \\
& \frac{2}{s}\left(\hat{p}_{2} \gamma^{i} \hat{p}_{1} \otimes \hat{p}_{1} B_{i}+\hat{p}_{2} \gamma^{i} \hat{p}_{1} \gamma_{5} \otimes \hat{p}_{1} \gamma_{5} B_{i}\right)=-\gamma^{i} \otimes \breve{B}_{i} \hat{p}_{1}-\gamma^{i} \gamma_{5} \otimes \breve{B}_{i} \hat{p}_{1} \gamma_{5} \\
& \gamma_{k} \gamma^{i} \hat{p}_{2} \otimes B_{i} \gamma^{k}+\gamma_{k} \gamma^{i} \hat{p}_{2} \gamma_{5} \otimes B_{i} \gamma^{k} \gamma_{5}=\hat{p}_{2} \otimes \gamma^{i} \breve{B}_{i}+\hat{p}_{2} \gamma_{5} \otimes \gamma_{i} \breve{B}^{i} \gamma_{5}, \\
& \not p_{2} \gamma^{j} \gamma^{i} \otimes \not p_{1} B_{j}+\not p_{2} \gamma_{5} \gamma^{i} \gamma^{j} \otimes \not p_{1} \gamma_{5} B_{j}=\not p_{2} \otimes \not p_{1} \breve{B}_{i}+\not p_{2} \gamma_{5} \otimes \not p_{1} \gamma_{5} \breve{B}^{i}, \\
& \not p_{2} \gamma^{i} \gamma^{j} \otimes \not p_{1} B_{j}+\not p_{2} \gamma_{5} \gamma^{i} \gamma^{j} \otimes \not p_{1} \gamma_{5} B_{j}=\not p_{2} \otimes \not p_{1} \breve{B}^{i}+\not p_{2} \gamma_{5} \otimes \not p_{1} \gamma_{5} \breve{B}^{i} \text {. }
\end{aligned}
$$

\section{A.1.4 Formulas with $\gamma$-matrices and two gluon fields}

With definition (A.18), we have the following formulas

$$
\begin{aligned}
& A_{i} \otimes \tilde{B}_{j}=g_{i j} \tilde{A}_{k} \otimes B^{k}-\tilde{A}_{j} \otimes B_{i}, \quad \tilde{A}_{i} \otimes B_{j}=g_{i j} A_{k} \otimes \tilde{B}^{k}-A_{j} \otimes \tilde{B}_{i} \\
& \tilde{A}_{i} \otimes \tilde{B}_{j}=-g_{i j} A_{k} \otimes B^{k}+A_{j} \otimes B_{i}, \quad \Rightarrow \tilde{A}_{i} \otimes \tilde{B}^{i}=-A_{i} \otimes B^{i}, \quad \tilde{A}_{i} \otimes B^{i}=A_{i} \otimes \tilde{B}^{i}
\end{aligned}
$$

In addition, it is convenient to define

$$
\breve{A}_{i} \equiv A_{i}-i \tilde{A}_{i} \gamma_{5}, \quad \breve{B}_{i} \equiv B_{i}-i \tilde{B}_{i} \gamma_{5} .
$$


Using these formulas, after some algebra one obtains

$\gamma_{m} \not p_{2} \gamma_{j} A^{i} \otimes \gamma_{n} \not p_{1} \gamma_{i} B^{j}+\gamma_{m} \not \not_{2} \gamma_{j} A^{i} \gamma_{5} \otimes \gamma_{n} \not p_{1} \gamma_{i} B^{j} \gamma_{5}=\not p_{2} \breve{A}_{n} \otimes \not p_{1} \breve{B}_{m}+\not p_{2} \breve{A}_{n} \gamma_{5} \otimes \not p_{1} \breve{B}_{m} \gamma_{5}$ $\gamma_{j} \not p_{2} \gamma_{m} A^{i} \otimes \gamma_{n} \not p_{1} \gamma_{i} B^{j}+\gamma_{j} \not p_{2} \gamma_{m} A^{i} \gamma_{5} \otimes \gamma_{n} \not p_{1} \gamma_{i} B^{j} \gamma_{5}=\not p_{2} \breve{A}_{n} \otimes \breve{B}_{m} \not p_{1}+\not p_{2} \breve{A}_{n} \gamma_{5} \otimes \breve{B}_{m} \not p_{1} \gamma_{5}$ $\gamma_{m} \not p_{2} \gamma_{j} A^{i} \otimes \gamma_{i} \not p_{1} \gamma_{n} B^{j}+\gamma_{m} \not p_{2} \gamma_{j} A^{i} \gamma_{5} \otimes \gamma_{i} \not p_{1} \gamma_{n} B^{j} \gamma_{5}=\breve{A}_{n} \not p_{2} \otimes \not p_{1} \breve{B}_{m}+\breve{A}_{n} \not p_{2} \gamma_{5} \otimes \not p_{1} \breve{B}_{m} \gamma_{5}$ $\gamma_{j} \not p_{2} \gamma_{m} A^{i} \otimes \gamma_{i} \not p_{1} \gamma_{n} B^{j}+\gamma_{j} \not p_{2} \gamma_{m} A^{i} \gamma_{5} \otimes \gamma_{i} \not p_{1} \gamma_{n} B^{j} \gamma_{5}=\breve{A}_{n} \not p_{2} \otimes \breve{B}_{m} \not p_{1}+\breve{A}_{n} \not p_{2} \gamma_{5} \otimes \breve{B}_{m} \not p_{1} \gamma_{5}$

and

$$
\begin{aligned}
& \not p_{2} \breve{A}_{m} \otimes \not p_{1} \breve{B}_{n}+\not p_{2} \breve{A}_{n} \gamma_{5} \otimes \not p_{1} \breve{B}_{m} \gamma_{5}=g_{m n} \not p_{2} \breve{A}_{k} \otimes \not p_{1} \breve{B}^{k} \\
& \not p_{2} \breve{A}_{m} \otimes \breve{B}_{n} \not p_{1}+\not p_{2} \breve{A}_{n} \gamma_{5} \otimes \gamma_{5} \breve{B}_{m} \not p_{1}=g_{m n} \not_{2} \breve{A}_{k} \otimes \breve{B}^{k} \not p_{1} \\
& \breve{A}_{m} \not p_{2} \otimes \not p_{1} \breve{B}_{n}+\gamma_{5} \breve{A}_{n} \not p_{2} \otimes \not p_{1} \breve{B}_{m} \gamma_{5}=g_{m n} \breve{A}_{k} \not p_{2} \otimes \not p_{1} \breve{B}^{k} \\
& \breve{A}_{m} \not p_{2} \otimes \breve{B}_{n} \not p_{1}+\gamma_{5} \breve{A}_{n} \not p_{2} \otimes \gamma_{5} \breve{B}_{m} \not p_{1}=g_{m n} \breve{A}_{k} \not p_{2} \otimes \breve{B}^{k} \not p_{1}
\end{aligned}
$$

The corollary of eq. (A.25) is

$$
\begin{aligned}
& \not p_{2} \breve{A}_{k} \gamma_{5} \otimes \not p_{1} \breve{B}^{k} \gamma_{5}=\not p_{2} \breve{A}_{k} \otimes \not p_{1} \breve{B}^{k}, \quad \not p_{2} \breve{A}_{k} \gamma_{5} \otimes \gamma_{5} \breve{B}^{k} \not p_{1}=\not p_{2} \breve{A}_{k} \otimes \breve{B}^{k} \not p_{1} \\
& \gamma_{5} \breve{A}_{k} \not p_{2} \otimes \not p_{1} \breve{B}^{k} \gamma_{5}=\breve{A}_{k} \not p_{2} \otimes \not p_{1} \breve{B}^{k}, \quad \gamma_{5} \breve{A}_{k} \not p_{2} \otimes \gamma_{5} \breve{B}^{k} \not p_{1}=\breve{A}_{k} \not p_{2} \otimes \breve{B}^{k} \not p_{1}
\end{aligned}
$$

From eqs. (A.24) and (A.25) one easily obtains

$$
\gamma_{m} \not p_{2} \gamma_{j} A^{i} \otimes \gamma_{n} \not p_{1} \gamma_{i} B^{j}+\gamma_{m} \not \not_{2} \gamma_{j} A^{i} \gamma_{5} \otimes \gamma_{n} \not p_{1} \gamma_{i} B^{j} \gamma_{5}+m \leftrightarrow n=2 g_{m n} \not p_{2} \breve{A}_{k} \otimes \not p_{1} \breve{B}^{k}
$$

and

$$
\begin{aligned}
& \gamma_{m} \not p_{2} \gamma_{j} A^{i} \otimes \gamma_{n} \not p_{1} \gamma_{i} B^{j}+\gamma_{m} \not p_{2} \gamma_{j} A^{i} \gamma_{5} \otimes \gamma_{n} \not p_{1} \gamma_{i} B^{j} \gamma_{5}-m \leftrightarrow n \\
& \quad=2 \not \not_{2} \breve{A}_{n} \otimes \not \not_{1} \breve{B}_{m}-m \leftrightarrow n, \\
& \gamma_{j} \not p_{2} \gamma_{m} A^{i} \otimes \gamma_{i} \not p_{1} \gamma_{n} B^{j}+\gamma_{j} \not p_{2} \gamma_{m} A^{i} \gamma_{5} \otimes \gamma_{i} \not p_{1} \gamma_{n} B^{j} \gamma_{5}-m \leftrightarrow n \\
& \quad=2 \breve{A}_{n} \not \breve{p}_{2} \otimes \breve{B}_{m} \not p_{1}-m \leftrightarrow n
\end{aligned}
$$

We need also the formula

$$
\begin{aligned}
& \frac{4}{s^{2}} A^{i} \not p_{1} \not p_{2} \gamma_{j} \otimes B^{j} \not p_{1} \not p_{2} \gamma_{i} \\
& =A^{i} \gamma_{j} \otimes B^{j} \gamma_{i}-i A^{i} \gamma_{j} \gamma_{5} \otimes \tilde{B}^{j} \gamma_{i}+i \tilde{A}^{i} \gamma_{j} \otimes B^{j} \gamma_{i} \gamma_{5}+\tilde{A}^{i} \gamma_{j} \gamma_{5} \otimes \tilde{B}^{j} \gamma_{i} \gamma_{5}
\end{aligned}
$$

and

$$
\begin{gathered}
\frac{4}{s^{2}}\left(A^{i} \not p_{1} \not p_{2} \gamma_{j} \otimes B^{j} \not p_{1} \not p_{2} \gamma_{i}+A^{i} \not p_{1} \not p_{2} \gamma_{j} \gamma_{5} \otimes B^{j} \not p_{1} \not p_{2} \gamma_{i} \gamma_{5}\right) \\
=\gamma^{j} \breve{A}_{i} \otimes \gamma^{i} \breve{B}_{j}+\gamma^{j} \breve{A}_{i} \gamma_{5} \otimes \gamma^{i} \breve{B}_{j} \gamma_{5} \\
\gamma_{i} \breve{A}_{j} \gamma_{5} \otimes \gamma_{j} \breve{A}_{i} \gamma_{5}=\gamma_{i} \breve{A}_{j} \otimes \gamma^{i} \breve{B}^{j}-\gamma_{i} \breve{A}^{i} \otimes \gamma_{j} \breve{B}^{j}
\end{gathered}
$$




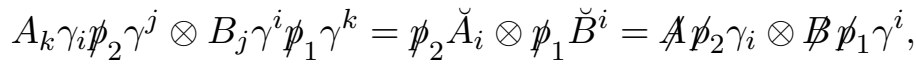

$A_{k} \gamma^{j} \not p_{2} \gamma_{i} \otimes B_{j} \gamma^{k} \not p_{1} \gamma^{i}=\breve{A}_{i} \not p_{2} \otimes \breve{B}_{i} \not p_{1}=\gamma_{i} \not p_{2} \not A \otimes \gamma^{i} \not p_{1} \not B$,

$A_{k} \gamma_{i} \not \not_{2} \gamma_{j} \otimes B^{j} \gamma^{k} \not p_{1} \gamma^{i}=\not p_{2} \breve{A}_{i} \otimes \breve{B}^{i} \not p_{1}=\not A \not p_{2} \gamma_{i} \otimes \gamma^{i} \not p_{1} \not B$,

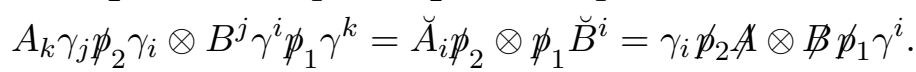

We used also

$$
\begin{aligned}
& A^{k} \gamma_{m} \not \not_{2} \gamma_{j} \otimes B^{j} \gamma_{n} \not \not_{1} \gamma_{k}+m \leftrightarrow n-g_{m n} A^{k} \gamma_{i} \not \not_{2} \gamma_{j} \otimes B^{j} \gamma^{i} \not p_{1} \gamma_{k} \\
& =\breve{A}_{m} \not p_{2} \otimes \breve{B}_{n} \not p_{1}+m \leftrightarrow n-g_{m n} \breve{A}_{k} \not p_{2} \otimes \breve{B}^{k} \not p_{1}, \\
& A^{k} \gamma_{j} \not p_{2} \gamma_{m} \otimes B^{j} \gamma_{n} \not \phi_{1} \gamma_{k}+m \leftrightarrow n-g_{m n} A^{k} \gamma_{j} \not p_{2} \gamma_{i} \otimes B^{j} \gamma_{k} \not p_{1} \gamma^{i} \\
& =p_{2} \breve{A}_{m} \otimes \breve{B}_{n} \not p_{1}+m \leftrightarrow n-g_{m n} \not p_{2} \breve{A}_{k} \otimes \breve{B}^{k} \not p_{1} \\
& A^{k} \gamma_{j} \not p_{2} \gamma_{m} \otimes B^{j} \gamma_{k} \not p_{1} \gamma_{n}+m \leftrightarrow n-g_{m n} A^{k} \gamma_{j} \not p_{2} \gamma_{i} \otimes B^{j} \gamma_{k} \not p_{1} \gamma^{i} \\
& =\not p_{2} \breve{A}_{m} \otimes \not p_{1} \breve{B}_{n}+m \leftrightarrow n-g_{m n} \not p_{2} \breve{A}_{k} \otimes \not p_{1} \breve{B}^{k} \\
& A^{k} \gamma_{m} \not p_{2} \gamma_{j} \otimes B^{j} \gamma_{k} \not p_{1} \gamma_{n}+m \leftrightarrow n-g_{m n} A^{k} \gamma_{j} \not p_{2} \gamma_{i} \otimes B^{j} \gamma_{k} \not_{1} \gamma^{i} \\
& =\breve{A}_{m} \not p_{2} \otimes \not p_{1} \breve{B}_{n}+m \leftrightarrow n-g_{m n} \breve{A}_{k} \not p_{2} \otimes \not p_{1} \breve{B}^{k} \text {, }
\end{aligned}
$$

and

$$
\begin{aligned}
& \gamma^{j} \not p_{2} \gamma_{m} A^{k} \otimes \gamma^{k} \not p_{1} \gamma_{n} B^{j}-m \leftrightarrow n=-\breve{A}_{m} \not p_{2} \otimes \breve{B}_{n} \not p_{1}-m \leftrightarrow n \\
& \gamma_{m} \not p_{2} \gamma^{j} A^{k} \otimes \gamma^{k} \not p_{1} \gamma_{n} B^{j}-m \leftrightarrow n=-\not \not_{2} \breve{A}_{m} \otimes \breve{B}_{n} \not p_{1}-m \leftrightarrow n \\
& \gamma^{j} \not p_{2} \gamma_{m} A^{k} \otimes \gamma_{n} \not p_{1} \gamma^{k} B^{j}-m \leftrightarrow n=-\breve{A}_{m} \not p_{2} \otimes \not p_{1} \breve{B}_{n}-m \leftrightarrow n \\
& \gamma_{m} \not p_{2} \gamma^{j} A^{k} \otimes \gamma_{n} \not p_{1} \gamma^{k} B^{j}-m \leftrightarrow n=-\not p_{2} \breve{A}_{m} \otimes \not p_{1} \breve{B}_{n}-m \leftrightarrow n
\end{aligned}
$$

Next, using formula (A.13) we get

$$
\begin{aligned}
& A^{i} \sigma_{\star j} \otimes B^{j} \sigma_{\bullet i}-A^{i} \sigma_{\star j} \gamma_{5} \otimes B^{j} \sigma_{\bullet i} \gamma_{5} \\
& =\left(\bar{A}^{j} \not p_{2} \gamma_{k}-\bar{A}^{k} \not p_{2} \gamma_{j}\right) \otimes \bar{B}^{j} \not p_{1} \gamma_{k}-\bar{A} \not p_{2} \otimes \bar{B} \not \not_{1}-\frac{s}{2} \bar{A} \gamma_{i} \otimes \overline{\not B} \gamma^{i}-\frac{s}{4} \bar{A}^{i} \sigma_{j k} \otimes \bar{B}_{i} \sigma^{j k}
\end{aligned}
$$

We frequently use this formula in matrix elements like $\left\langle\bar{\psi} A^{i}(x) \sigma_{\star j} \frac{1}{\alpha} \psi(0)\right\rangle_{A}$ $\left\langle\bar{\psi} B^{j}(0) \sigma_{\bullet} i \frac{1}{\beta} \psi(x)\right\rangle_{B}$. It is easy to see that in such matrix elements the contribution of two last terms in eq. (A.34) is $O\left(\frac{q_{\perp}^{2}}{s}\right)$ in comparison to the first two ones so for our purposes

$$
A^{i} \sigma_{\star j} \otimes B^{j} \sigma_{\bullet i}-A^{i} \sigma_{\star j} \gamma_{5} \otimes B^{j} \sigma_{\bullet i} \gamma_{5} \simeq\left(\bar{A}^{j} \not p_{2} \gamma_{k}-\bar{A}^{k} \not p_{2} \gamma_{j}\right) \otimes \bar{B}^{j} \not p_{1} \gamma_{k}-\bar{A} \not p_{2} \otimes \bar{B} \not p_{1}
$$

We also need

$$
\begin{aligned}
& \frac{4}{s^{2}}\left[A_{i} \not p_{1} \not p_{2} \gamma_{j} \otimes B_{j} \not \not_{2} \not p_{1} \gamma_{i}+A_{i} \not \not_{1} \not p_{2} \gamma_{j} \gamma_{5} \otimes B_{j} \not \not_{2} \not p_{1} \gamma_{i} \gamma_{5}\right] \\
& =\breve{A}_{i} \gamma_{j} \otimes \breve{B}_{j} \gamma_{i}+\breve{A}_{i} \gamma_{j} \gamma_{5} \otimes \breve{B}_{j} \gamma_{i} \gamma_{5}
\end{aligned}
$$


and

$$
\begin{aligned}
& \frac{2}{s}\left[A_{i} \not p_{1} \not p_{2} \gamma^{j} \otimes B_{j} \gamma_{n} \not p_{1} \gamma^{i}+A_{i} \not p_{1} \not p_{2} \gamma^{j} \gamma_{5} \otimes B_{j} \gamma_{\nu_{\perp}} \not p_{1} \gamma^{i} \gamma_{5}\right] \\
& =-\gamma_{i} \breve{A}_{n} \otimes \not p_{1} \breve{B}^{i}-\gamma_{i} \breve{A}_{n} \gamma_{5} \otimes \not p_{1} \breve{B}^{i} \gamma_{5}=\gamma_{i} \breve{A}_{n} \otimes \not B \not p_{1} \gamma^{i}+\gamma_{i} \breve{A}_{n} \gamma_{5} \otimes \not B \not p_{1} \gamma^{i} \gamma_{5} \text {, } \\
& \frac{2}{s}\left[A_{i} \gamma_{n} \not p_{2} \gamma^{j} \otimes B_{j} \not p_{2} \not p_{1} \gamma^{i}+A_{i} \gamma_{n} \not p_{2} \gamma^{j} \gamma_{5} \otimes B_{j} \not p_{2} \not p_{1} \gamma^{i} \gamma_{5}\right] \\
& =-\not p_{2} \breve{A}_{i} \otimes \gamma^{i} \breve{B}_{n}-\not p_{2} \breve{A}_{i} \gamma_{5} \otimes \gamma^{i} \breve{B}_{n} \gamma_{5}=\not A p_{2} \gamma_{i} \otimes \gamma^{i} \breve{B}_{n}+\not A p_{2} \gamma_{i} \gamma_{5} \otimes \gamma^{i} \breve{B}_{n} \gamma_{5} \text {. }
\end{aligned}
$$

The last formula which we need is

$\gamma_{m} \not p_{2} \gamma_{j} A^{i} \otimes \gamma_{n} \not p_{1} \gamma_{i} B^{j}+\gamma_{m} \not \not_{2} \gamma_{j} A^{i} \gamma_{5} \otimes \gamma_{n} \not p_{1} \gamma_{i} B^{j} \gamma_{5}=\not p_{2} \breve{A}_{n} \otimes \not p_{1} \breve{B}_{m}+\not p_{2} \breve{A}_{n} \gamma_{5} \otimes \not p_{1} \breve{B}_{m} \gamma_{5}$

$\gamma_{j} \not p_{2} \gamma_{m} A^{i} \otimes \gamma_{n} \not p_{1} \gamma_{i} B^{j}+\gamma_{j} \not p_{2} \gamma_{m} A^{i} \gamma_{5} \otimes \gamma_{n} \not p_{1} \gamma_{i} B^{j} \gamma_{5}=\not p_{2} \breve{A}_{n} \otimes \breve{B}_{m} \not p_{1}+\not p_{2} \breve{A}_{n} \gamma_{5} \otimes \breve{B}_{m} \not p_{1} \gamma_{5}$ $\gamma_{m} \not p_{2} \gamma_{j} A^{i} \otimes \gamma_{i} \not p_{1} \gamma_{n} B^{j}+\gamma_{m} \not p_{2} \gamma_{j} A^{i} \gamma_{5} \otimes \gamma_{i} \not p_{1} \gamma_{n} B^{j} \gamma_{5}=\breve{A}_{n} \not p_{2} \otimes \not p_{1} \breve{B}_{m}+\breve{A}_{n} \not p_{2} \gamma_{5} \otimes \not p_{1} \breve{B}_{m} \gamma_{5}$ $\gamma_{j} \not p_{2} \gamma_{m} A^{i} \otimes \gamma_{i} \not p_{1} \gamma_{n} B^{j}+\gamma_{j} \not p_{2} \gamma_{m} A^{i} \gamma_{5} \otimes \gamma_{i} \not p_{1} \gamma_{n} B^{j} \gamma_{5}=\breve{A}_{n} \not p_{2} \otimes \breve{B}_{m} \not p_{1}+\breve{A}_{n} \not p_{2} \gamma_{5} \otimes \breve{B}_{m} \not p_{1} \gamma_{5}$

\section{A.2 Parametrization of leading-twist matrix elements}

Let us first consider matrix elements of operators without $\gamma_{5}$. The standard parametrization of quark TMDs reads

$$
\begin{aligned}
& \frac{1}{16 \pi^{3}} \int d x_{\bullet} d^{2} x_{\perp} e^{-i \alpha x_{\bullet}+i(k, x)_{\perp}}\left\langle\hat{\bar{\psi}}_{f}\left(x_{\bullet}, x_{\perp}\right) \gamma^{\mu} \hat{\psi}_{f}(0)\right\rangle_{A} \\
& =p_{1}^{\mu} f_{1}^{f}\left(\alpha, k_{\perp}\right)+k_{\perp}^{\mu} f_{\perp}^{f}\left(\alpha, k_{\perp}\right)+p_{2}^{\mu} \frac{2 m_{N}^{2}}{s} f_{3}^{f}\left(\alpha, k_{\perp}\right), \\
& \frac{1}{16 \pi^{3}} \int d x_{\bullet} d^{2} x_{\perp} e^{-i \alpha x_{\bullet}+i(k, x)_{\perp}}\left\langle\hat{\bar{\psi}}_{f}\left(x_{\bullet}, x_{\perp}\right) \hat{\psi}_{f}(0)\right\rangle_{A}=m_{N} e^{f}\left(\alpha, k_{\perp}\right)
\end{aligned}
$$

for quark distributions in the projectile and

$$
\begin{aligned}
& \frac{1}{16 \pi^{3}} \int d x_{\bullet} d^{2} x_{\perp} e^{-i \alpha x_{\bullet}+i(k, x)_{\perp}}\left\langle\hat{\bar{\psi}}_{f}(0) \gamma^{\mu} \hat{\psi}_{f}\left(x_{\bullet}, x_{\perp}\right)\right\rangle_{A} \\
& =-p_{1}^{\mu} \bar{f}_{1}^{f}\left(\alpha, k_{\perp}\right)-k_{\perp}^{\mu} \bar{f}_{\perp}^{f}\left(\alpha, k_{\perp}\right)-p_{2}^{\mu} \frac{2 m_{N}^{2}}{s} \bar{f}_{3}^{f}\left(\alpha, k_{\perp}\right), \\
& \frac{1}{16 \pi^{3}} \int d x_{\bullet} d^{2} x_{\perp} e^{-i \alpha x_{\bullet}+i(k, x)_{\perp}}\left\langle\hat{\bar{\psi}}_{f}(0) \hat{\psi}_{f}\left(x_{\bullet}, x_{\perp}\right)\right\rangle_{A}=m_{N} \bar{e}^{f}\left(\alpha, k_{\perp}\right)
\end{aligned}
$$

for the antiquark distributions. ${ }^{11}$

\footnotetext{
${ }^{11}$ In a general gauge for projectile and target fields these matrix elements read

$$
\begin{aligned}
& \left\langle\hat{\psi}_{f}(x) \gamma_{\mu} \hat{\psi}_{f}(0)\right\rangle_{A}=\left\langle\hat{\psi}_{f}\left(x_{\bullet}, x_{\perp}\right) \gamma_{\mu}\left[x_{\bullet},-\infty_{\bullet}\right]_{x}\left[x_{\perp}, 0_{\perp}\right]_{-\infty}\left[-\infty_{\bullet}, 0_{\bullet}\right]_{0} \hat{\psi}_{f}(0)\right\rangle_{A}, \\
& \left\langle\hat{\psi}_{f}(x) \gamma_{\mu} \hat{\psi}_{f}(0)\right\rangle_{B}=\left\langle\hat{\psi}_{f}\left(x_{*}, x_{\perp}\right) \gamma_{\mu}\left[x_{*},-\infty_{*}\right]_{x}\left[x_{\perp}, 0_{\perp}\right]_{-\infty_{*}}\left[-\infty_{*}, 0_{*}\right]_{0} \hat{\psi}_{f}(0)\right\rangle_{B}
\end{aligned}
$$
}

and similarly for other operators. 
The corresponding matrix elements for the target are obtained by trivial replacements $p_{1} \leftrightarrow p_{2}, x_{\bullet} \leftrightarrow x_{\star}$ and $\alpha \leftrightarrow \beta$ :

$$
\begin{aligned}
& \frac{1}{16 \pi^{3}} \int d x_{\star} d^{2} x_{\perp} e^{-i \beta x_{\star}+i(k, x)_{\perp}}\left\langle\hat{\bar{\psi}}_{f}\left(x_{\star}, x_{\perp}\right) \gamma^{\mu} \hat{\psi}_{f}(0)\right\rangle_{B} \\
& =p_{2}^{\mu} f_{1}^{f}\left(\beta, k_{\perp}\right)+k_{\perp}^{\mu} f_{\perp}^{f}\left(\beta, k_{\perp}\right)+p_{1}^{\mu} \frac{2 m_{N}^{2}}{s} f_{3}^{f}\left(\beta, k_{\perp}\right), \\
& \frac{1}{16 \pi^{3}} \int d x_{\star} d^{2} x_{\perp} e^{-i \beta x_{\star}+i(k, x)_{\perp}}\left\langle\hat{\bar{\psi}}_{f}\left(x_{\star}, x_{\perp}\right) \hat{\psi}_{f}(0)\right\rangle_{B}=m_{N} e^{f}\left(\beta, k_{\perp}\right),
\end{aligned}
$$

and

$$
\begin{aligned}
& \frac{1}{16 \pi^{3}} \int d x_{\star} d^{2} x_{\perp} e^{-i \beta x_{\star}+i(k, x)_{\perp}}\left\langle\hat{\bar{\psi}}_{f}(0) \gamma^{\mu} \hat{\psi}_{f}\left(x_{\star}, x_{\perp}\right)\right\rangle_{B} \\
& =-p_{2}^{\mu} \bar{f}_{1}^{f}\left(\beta, k_{\perp}\right)-k_{\perp}^{\mu} \bar{f}_{\perp}^{f}\left(\beta, k_{\perp}\right)-p_{1}^{\mu} \frac{2 m_{N}^{2}}{s} \bar{f}_{3}^{f}\left(\beta, k_{\perp}\right), \\
& \frac{1}{16 \pi^{3}} \int d x_{\star} d^{2} x_{\perp} e^{-i \beta x_{\star}+i(k, x)_{\perp}}\left\langle\hat{\bar{\psi}}_{f}(0) \hat{\psi}_{f}\left(x_{\star}, x_{\perp}\right)\right\rangle_{B}=m_{N} \bar{e}^{f}\left(\beta, k_{\perp}\right) .
\end{aligned}
$$

Matrix elements of operators with $\gamma_{5}$ are parametrized as follows:

$$
\begin{aligned}
& \frac{1}{16 \pi^{3}} \int d x_{\bullet} d^{2} x_{\perp} e^{-i \alpha x_{\bullet}+i(k, x)_{\perp}}\left\langle\hat{\bar{\psi}}_{f}\left(x_{\bullet}, x_{\perp}\right) \gamma^{\mu} \gamma_{5} \hat{\psi}_{f}(0)\right\rangle_{A}=-i \epsilon_{\mu_{\perp} i} k^{i} g_{f}^{\perp}\left(\alpha, k_{\perp}\right), \\
& \frac{1}{16 \pi^{3}} \int d x_{\bullet} d^{2} x_{\perp} e^{-i \alpha x_{\bullet}+i(k, x)_{\perp}}\left\langle\hat{\bar{\psi}}_{f}(0) \gamma^{\mu} \gamma_{5} \hat{\psi}_{f}\left(x_{\bullet}, x_{\perp}\right)\right\rangle_{A}=-i \epsilon_{\mu_{\perp} i} k^{i} \bar{g}_{f}^{\perp}\left(\alpha, k_{\perp}\right)
\end{aligned}
$$

The corresponding matrix elements for the target are obtained by trivial replacements $p_{1} \leftrightarrow p_{2}, x_{\bullet} \leftrightarrow x_{\star}$ and $\alpha \leftrightarrow \beta$ similarly to eq. (A.42).

The parametrization of time-odd Boer-Mulders TMDs are

$$
\begin{aligned}
& \frac{1}{16 \pi^{3}} \int d x_{\bullet} d^{2} x_{\perp} e^{-i \alpha x_{\bullet}+i(k, x)_{\perp}}\left\langle\hat{\bar{\psi}}_{f}\left(x_{\bullet}, x_{\perp}\right) \sigma^{\mu \nu} \hat{\psi}_{f}(0)\right\rangle_{A} \\
& =\frac{1}{m_{N}}\left(k_{\perp}^{\mu} p_{1}^{\nu}-\mu \leftrightarrow \nu\right) h_{1 f}^{\perp}\left(\alpha, k_{\perp}\right)+\frac{2 m_{N}}{s}\left(p_{1}^{\mu} p_{2}^{\nu}-\mu \leftrightarrow \nu\right) h_{f}\left(\alpha, k_{\perp}\right) \\
& \quad+\frac{2 m_{N}}{s}\left(k_{\perp}^{\mu} p_{2}^{\nu}-\mu \leftrightarrow \nu\right) h_{3 f}^{\perp}\left(\alpha, k_{\perp}\right), \\
& \frac{1}{16 \pi^{3}} \int d x_{\bullet} d^{2} x_{\perp} e^{-i \alpha x_{\bullet}+i(k, x)_{\perp}}\left\langle\hat{\bar{\psi}}_{f}(0) \sigma^{\mu \nu} \hat{\psi}_{f}\left(x_{\bullet}, x_{\perp}\right)\right\rangle_{A} \\
& =-\frac{1}{m_{N}}\left(k_{\perp}^{\mu} p_{1}^{\nu}-\mu \leftrightarrow \nu\right) \bar{h}_{1 f}^{\perp}\left(\alpha, k_{\perp}\right)-\frac{2 m_{N}}{s}\left(p_{1}^{\mu} p_{2}^{\nu}-\mu \leftrightarrow \nu\right) \bar{h}_{f}\left(\alpha, k_{\perp}\right) \\
& \quad-\frac{2 m_{N}}{s}\left(k_{\perp}^{\mu} p_{2}^{\nu}-\mu \leftrightarrow \nu\right) \bar{h}_{3 f}^{\perp}\left(\alpha, k_{\perp}\right)
\end{aligned}
$$

and similarly for the target with usual replacements $p_{1} \leftrightarrow p_{2}, x_{\bullet} \leftrightarrow x_{\star}$ and $\alpha \leftrightarrow \beta$.

Note that the coefficients in front of $f_{3}, g_{f}^{\perp}, h$ and $h_{3}^{\perp}$ in eqs. (A.39), (A.41), (A.43), and (A.44) contain an extra $\frac{1}{s}$ since $p_{2}^{\mu}$ enters only through the direction of gauge link so the result should not depend on rescaling $p_{2} \rightarrow \lambda p_{2}$. For this reason, these functions do not contribute to $W(q)$ in our approximation. 
Last but not least, an important point in our analysis is that any $f\left(x, k_{\perp}\right)$ may have only logarithmic dependence on Bjorken $x$ but not the power dependence $\sim \frac{1}{x}$. Indeed, at small $x$ the cutoff of corresponding longitudinal integrals comes from the rapidity cutoff $\sigma_{a}$, see footnote 12 and corresponding discussion in ref. [16]. Thus, at small $x$ one can safely put $x=0$ and the corresponding logarithmic contributions would be proportional to powers of $\alpha_{s} \ln \sigma_{a}$ (or, in some cases, $\alpha_{s} \ln ^{2} \sigma_{a}$, see e.g. ref. [35]).

\section{A.3 Matrix elements of quark-quark-gluon operators}

In this section we will demonstrate that matrix elements of quark-antiquark-gluon operators from section 4 can be expressed in terms of leading-power matrix elements from section A.2. First, let us note that operators $\frac{1}{\alpha}$ and $\frac{1}{\beta}$ in eqs. (3.5) are replaced by $\pm \frac{1}{\alpha_{q}}$ and $\pm \frac{1}{\beta_{q}}$ in forward matrix elements. Indeed

$$
\begin{aligned}
& \int d x_{\bullet} e^{-i \alpha_{q} x_{\bullet}}\left\langle\bar{\Phi}\left(x_{\bullet}, x_{\perp}\right) \Gamma \frac{1}{\alpha+i \epsilon} \psi(0)\right\rangle_{A} \\
& =-i \int d x_{\bullet} \int_{-\infty}^{0} d x_{\bullet}^{\prime} e^{-i \alpha_{q} x_{\bullet}}\left\langle\bar{\Phi}\left(x_{\bullet}, x_{\perp}\right) \Gamma \psi\left(x_{\bullet}^{\prime}, 0_{\perp}\right)\right\rangle_{A}=\frac{1}{\alpha_{q}} \int d x_{\bullet} e^{-i \alpha x} \bullet\left\langle\bar{\Phi}\left(x_{\bullet}, x_{\perp}\right) \Gamma \psi(0)\right\rangle_{A}
\end{aligned}
$$

where $\bar{\Phi}\left(x_{\bullet}, x_{\perp}\right)$ can be $\bar{\psi}\left(x_{\bullet}, x_{\perp}\right)$ or $\bar{\psi}\left(x_{\bullet}, x_{\perp}\right) A_{i}\left(x_{\bullet}, x_{\perp}\right)$ and $\Gamma$ can be any $\gamma$-matrix. Similarly,

$$
\begin{aligned}
\int d x_{\bullet} e^{-i \alpha_{q} x_{\bullet}}\left\langle\left(\bar{\psi}\left(x_{\bullet}, x_{\perp}\right) \frac{1}{\alpha-i \epsilon}\right) \Gamma \Phi(0)\right\rangle_{A} & =\frac{1}{\alpha_{q}} \int d x_{\bullet} e^{-i \alpha x_{\bullet}}\left\langle\bar{\psi}\left(x_{\bullet}, x_{\perp}\right) \Gamma \Phi(0)\right\rangle_{A} \\
\int d x_{\bullet} e^{-i \alpha_{q} x_{\bullet}}\left\langle\left(\bar{\psi} \frac{1}{\alpha-i \epsilon}\right)\left(x_{\bullet}, x_{\perp}\right) \Gamma \frac{1}{\alpha+i \epsilon} \psi(0)\right\rangle_{A} & =\frac{1}{\alpha_{q}^{2}} \int d x_{\bullet} e^{-i \alpha_{q} x_{\bullet}}\left\langle\bar{\psi}\left(x_{\bullet}, x_{\perp}\right) \Gamma \psi(0)\right\rangle_{A}
\end{aligned}
$$

where $\Phi\left(x_{\bullet}, x_{\perp}\right)$ can be $\psi\left(x_{\bullet}, x_{\perp}\right)$ or $A_{i}\left(x_{\bullet}, x_{\perp}\right) \psi\left(x_{\bullet}, x_{\perp}\right)$. We need also

$$
\begin{aligned}
\int d x_{\bullet} e^{-i \alpha_{q} x_{\bullet}}\left\langle\left(\bar{\psi}(0) \frac{1}{\alpha-i \epsilon}\right) \Gamma \Phi\left(x_{\bullet}, x_{\perp}\right)\right\rangle_{A} & =-\frac{1}{\alpha_{q}} \int d x_{\bullet} e^{-i \alpha x_{\bullet}}\left\langle\bar{\psi}() \Gamma \Phi\left(x_{\bullet}, x_{\perp}\right)\right\rangle_{A} \\
\int d x_{\bullet} e^{-i \alpha_{q} x}\left\langle\bar{\Phi}(0) \Gamma \frac{1}{\alpha+i \epsilon} \psi\left(x_{\bullet}, x_{\perp}\right)\right\rangle_{A} & =-\frac{1}{\alpha_{q}} \int d x_{\bullet} e^{-i \alpha x} \bullet\left\langle\bar{\Phi}(0) \Gamma \psi\left(x_{\bullet}, x_{\perp}\right)\right\rangle_{A}
\end{aligned}
$$

The corresponding formulas for target matrix elements are obtained by substitution $\alpha \leftrightarrow \beta$ (and $x_{\bullet} \leftrightarrow x_{\star}$ ).

Next, we will use QCD equation of motion to reduce quark-quark-gluon TMDs to leading-twist TMDs (cf. ref. [20]). Let us start with matrix element

$$
\begin{aligned}
\int d x_{\bullet} d x_{\perp} e^{-i \alpha_{q} x_{\bullet}+i(k, x)_{\perp}}\left\langle\bar{\psi}\left(x_{\bullet}, x_{\perp}\right) \not p_{2} \breve{A}_{i}\left(x_{\bullet}, x_{\perp}\right) \psi(0)\right\rangle_{A} \\
=-\int d x_{\bullet} d x_{\perp} e^{-i \alpha_{q} x_{\bullet}+i(k, x)_{\perp}}\left\langle\bar{\psi}\left(x_{\bullet}, x_{\perp}\right) \not A\left(x_{\bullet}, x_{\perp}\right) \not p_{2} \gamma_{i} \psi(0)\right\rangle_{A} \\
=\int d x_{\bullet} d x_{\perp} e^{-i \alpha_{q} x_{\bullet}+i(k, x)_{\perp}} \\
\quad \times \quad\left[\left\langle\hat{\bar{\psi}}\left(x_{\bullet}, x_{\perp}\right) \not k_{\perp} \not p_{2} \gamma_{i} \hat{\psi}(0)\right\rangle_{A}+i\left\langle\hat{\bar{\psi}}\left(x_{\bullet}, x_{\perp}\right) \overleftarrow{D D}_{\perp} \gamma_{j} \not p_{2} \gamma_{i} \hat{\psi}(0)\right\rangle_{A}\right]
\end{aligned}
$$


Using QCD equations of motion we can rewrite the r.h.s. of eq. (A.48) as

$$
\begin{aligned}
\int d x_{\bullet} d x_{\perp} e^{-i \alpha_{q} x_{\bullet}+i(k, x)_{\perp}}\left[\left\langle\hat{\bar{\psi}}\left(x_{\bullet}, x_{\perp}\right) \not k_{\perp} \not p_{2} \gamma_{i} \hat{\psi}(0)\right\rangle_{A}+\alpha_{q}\left\langle\hat{\bar{\psi}}\left(x_{\bullet}, x_{\perp}\right) \not p_{1} \not p_{2} \gamma_{i} \hat{\psi}(0)\right\rangle_{A}\right] \\
=\int d x_{\bullet} d x_{\perp} e^{-i \alpha_{q} x_{\bullet}+i(k, x)_{\perp}}\left[-k_{i}\left\langle\hat{\bar{\psi}}\left(x_{\bullet}, x_{\perp}\right) \not p_{2} \hat{\psi}(0)\right\rangle_{A}+\alpha_{q} \frac{s}{2}\left\langle\hat{\bar{\psi}}\left(x_{\bullet}, x_{\perp}\right) \gamma_{i} \hat{\psi}(0)\right\rangle_{A}\right. \\
\left.\quad-i \epsilon_{i j} k^{j}\left\langle\hat{\bar{\psi}}\left(x_{\bullet}, x_{\perp}\right) \not p_{2} \gamma_{5} \hat{\psi}(0)\right\rangle_{A}+i \frac{s}{2} \alpha \epsilon_{i j}\left\langle\hat{\bar{\psi}}\left(x_{\bullet}, x_{\perp}\right) \gamma^{j} \gamma_{5} \hat{\psi}(0)\right\rangle_{A}\right] \\
=-k_{i} 8 \pi^{3} s f_{1}\left(\alpha_{q}, k_{\perp}\right)+8 \pi^{3} s \alpha_{q} k_{i}\left[f_{\perp}\left(\alpha_{q}, k_{\perp}\right)+g^{\perp}\left(\alpha_{q}, k_{\perp}\right)\right]
\end{aligned}
$$

where we used parametrizations (A.39) and (A.43) for the leading power matrix elements.

Now, the second term in eq. (A.49) contains extra $\alpha_{q}$ with respect to the first term, ${ }^{12}$ so it should be neglected in our kinematical region $s \gg Q^{2} \gg q_{\perp}^{2}$ and we get

$$
\begin{aligned}
& \frac{g}{8 \pi^{3} s} \int d x_{\bullet} d x_{\perp} e^{-i \alpha_{q} x_{\bullet}+i(k, x)_{\perp}}\left\langle\bar{\psi}^{f}\left(x_{\bullet}, x_{\perp}\right) \not p_{2} \breve{A}_{i}\left(x_{\bullet}, x_{\perp}\right) \psi^{f}(0)\right\rangle_{A} \\
& =\frac{-g}{8 \pi^{3} s} \int d x_{\bullet} d x_{\perp} e^{-i \alpha_{q} x_{\bullet}+i(k, x) \perp}\left\langle\bar{\psi}^{f}\left(x_{\bullet}, x_{\perp}\right) A\left(x_{\bullet}, x_{\perp}\right) \not p_{2} \gamma_{i} \psi^{f}(0)\right\rangle_{A}=-k_{i} f_{1}^{f}\left(\alpha_{q}, k_{\perp}\right)
\end{aligned}
$$

By complex conjugation

$$
\begin{aligned}
& \frac{g}{8 \pi^{3} s} \int d x_{\perp} d x_{\bullet} e^{-i \alpha_{q} x_{\bullet}+i(k, x)_{\perp}}\left\langle\hat{\bar{\psi}}_{f}\left(x_{\bullet}, x_{\perp}\right) \breve{A}_{i}(0) \not p_{2} \hat{\psi}_{f}(0)\right\rangle_{A} \\
& =\frac{-g}{8 \pi^{3} s} \int d x_{\bullet} d x_{\perp} e^{-i \alpha_{q} x_{\bullet}+i(k, x)_{\perp}}\left\langle\bar{\psi}^{f}\left(x_{\bullet}, x_{\perp}\right) \gamma_{i} \not p_{2} \not A(0) \psi^{f}(0)\right\rangle_{A}=-k_{i} f_{1 f}\left(\alpha_{q}, k_{\perp}\right) .
\end{aligned}
$$

For the corresponding antiquark distributions we get

$$
\begin{aligned}
& \frac{g}{8 \pi^{3} s} \int d x_{\perp} d x_{\bullet} e^{-i \alpha x_{\bullet}+i(k, x)_{\perp}}\left\langle\bar{\psi}_{f}(0) \breve{A}_{i}\left(x_{\bullet}, x_{\perp}\right) \not p_{2} \hat{\psi}_{f}\left(x_{\bullet}, x_{\perp}\right)\right\rangle_{A} \\
& =\frac{1}{8 \pi^{3} s} \int d x_{\bullet} d x_{\perp} e^{-i \alpha_{q} x \bullet+i(k, x)_{\perp}}\left[-\left\langle\hat{\bar{\psi}}(0) \gamma_{i} \not p_{2} k_{\perp} \hat{\psi}\left(x_{\bullet}, x_{\perp}\right)\right\rangle_{A}\right. \\
& \left.\quad-i\left\langle\hat{\bar{\psi}}(0) \gamma_{i} \not p_{2} \not D_{\perp} \hat{\psi}\left(x_{\bullet}, x_{\perp}\right)\right\rangle_{A}\right]=-k_{i} \bar{f}_{1 f}\left(\alpha_{q}, k_{\perp}\right)
\end{aligned}
$$

and

$$
\frac{g}{8 \pi^{3} s} \int d x_{\perp} d x_{\bullet} e^{-i \alpha_{q} x_{\bullet}+i(k, x)_{\perp}}\left\langle\hat{\bar{\psi}}_{f}(0) \not p_{2} \breve{A}_{i}(0) \hat{\psi}_{f}\left(x_{\bullet}, x_{\perp}\right)\right\rangle_{A}=-k_{i} \bar{f}_{1 f}\left(\alpha_{q}, k_{\perp}\right) .
$$

The corresponding target matrix elements are obtained by trivial replacements $x_{\star} \leftrightarrow$ $x_{\bullet}, \alpha_{q} \leftrightarrow \beta_{q}$ and $\not p_{2} \leftrightarrow \not p_{1}$.

Next, let us consider

$$
\begin{aligned}
& \frac{g}{8 \pi^{3} s} \int d x_{\bullet} d x_{\perp} e^{-i \alpha_{q} x_{\bullet}+i(k, x)_{\perp}}\left\langle\hat{\psi}\left(x_{\bullet}, x_{\perp}\right) \not p_{2} A\left(x_{\bullet}, x_{\perp}\right) \hat{\psi}(0)\right\rangle_{A} \\
& =\frac{1}{8 \pi^{3} s} \int d x_{\bullet} d x_{\perp} e^{-i \alpha_{q} x_{\bullet}+i(k, x)_{\perp}} \\
& \quad \times\left[\left\langle\hat{\psi}\left(x_{\bullet}, x_{\perp}\right) \not k_{\perp} \not p_{2} \hat{\psi}(0)\right\rangle_{A}+i\left\langle\hat{\psi}\left(x_{\bullet}, x_{\perp}\right) \overleftarrow{D D}_{\perp} \not \not_{2} \hat{\psi}(0)\right\rangle_{A}\right] .
\end{aligned}
$$

\footnotetext{
${ }^{12}$ It can be demonstrated that $f_{1}\left(x, k_{\perp}^{2}\right), f_{\perp}\left(x, k_{\perp}^{2}\right)$, and $f_{\perp}\left(x, k_{\perp}^{2}\right)$ have the same type of (logarithmic) behavior at low $x$. Indeed, the low- $x$ behavior is determined by interaction with pomeron. This interaction is specified by so-called impact factor and it is easy to check that the impact factor for all three TMDs is similar.
} 
Using QCD equation of motion and parametrization (A.44), one can rewrite the r.h.s. of this equation as

$$
\begin{aligned}
& \frac{1}{8 \pi^{3} s} \int d x_{\bullet} d x_{\perp} e^{-i \alpha_{q} x_{\bullet}+i(k, x) \perp}\left[\left\langle\hat{\bar{\psi}}\left(x_{\bullet}, x_{\perp}\right) \not k_{\perp} \not p_{2} \hat{\psi}(0)\right\rangle_{A}+\alpha_{q}\left\langle\hat{\bar{\psi}}\left(x_{\bullet}, x_{\perp}\right) \not p_{1} \not p_{2} \hat{\psi}(0)\right\rangle_{A}\right] \\
& =i \frac{k_{\perp}^{2}}{m_{N}} h_{1}^{\perp}\left(\alpha_{q}, k_{\perp}\right)+\alpha_{q} m_{N}\left[e\left(\alpha, k_{\perp}\right)+i h\left(\alpha, k_{\perp}\right)\right] .
\end{aligned}
$$

Again, only the first term contributes in our kinematical region so we finally get

$$
\frac{g}{8 \pi^{3} s} \int d x_{\bullet} d x_{\perp} e^{-i \alpha_{q} x_{\bullet}+i(k, x) \perp}\left\langle\hat{\bar{\psi}}^{f}\left(x_{\bullet}, x_{\perp}\right) \not p_{2} \not A\left(x_{\bullet}, x_{\perp}\right) \hat{\psi}^{f}(0)\right\rangle_{A}=i \frac{k_{\perp}^{2}}{m_{N}} h_{1 f}^{\perp}\left(\alpha_{q}, k_{\perp}\right) .
$$

By complex conjugation we obtain

$$
\frac{g}{8 \pi^{3} s} \int d x_{\bullet} d x_{\perp} e^{-i \alpha_{q} x_{\bullet}+i(k, x)_{\perp}}\left\langle\hat{\bar{\psi}}^{f}\left(x_{\bullet}, x_{\perp}\right) \not p_{2} \mathcal{A}(0) \hat{\psi}^{f}(0)\right\rangle_{A}=i \frac{k_{\perp}^{2}}{m_{N}} h_{1 f}^{\perp}\left(\alpha_{q}, k_{\perp}\right) .
$$

For corresponding antiquark distributions one gets in a similar way

$$
\begin{aligned}
\frac{g}{8 \pi^{3} s} \int d x_{\bullet} d x_{\perp} e^{-i \alpha_{q} x_{\bullet}+i(k, x)_{\perp}}\left\langle\hat{\bar{\psi}}^{f}(0) \not p_{2} \not A\left(x_{\bullet}, x_{\perp}\right) \hat{\psi}^{f}\left(x_{\bullet}, x_{\perp}\right)\right\rangle_{A} & =i \frac{k_{\perp}^{2}}{m_{N}} \bar{h}_{1 f}^{\perp}\left(\alpha_{q}, k_{\perp}\right), \\
\frac{g}{8 \pi^{3} s} \int d x_{\bullet} d x_{\perp} e^{-i \alpha_{q} x \bullet+i(k, x)_{\perp}}\left\langle\hat{\bar{\psi}}^{f}(0) \not p_{2} A(0) \hat{\psi}^{f}\left(x_{\bullet}, x_{\perp}\right)\right\rangle_{A} & =i \frac{k_{\perp}^{2}}{m_{N}} \bar{h}_{1 f}^{\perp}\left(\alpha_{q}, k_{\perp}\right) .
\end{aligned}
$$

The target matrix elements are obtained by usual replacements $x_{\star} \leftrightarrow x_{\bullet}, \alpha_{q} \leftrightarrow \beta_{q}$ and $\not p_{2} \leftrightarrow \not p_{1}$.

For the Z-boson hadronic tensor we need these operators with extra $\gamma_{5}$. From formula (A.49) we see that

$$
\begin{gathered}
\frac{1}{8 \pi^{3} s} \int d x_{\bullet} d x_{\perp} e^{-i \alpha_{q} x_{\bullet}+i(k, x)_{\perp}}\left\langle\bar{\psi}^{f}\left(x_{\bullet}, x_{\perp}\right) \not p_{2} \breve{A}_{i}\left(x_{\bullet}, x_{\perp}\right) \gamma_{5} \psi^{f}(0)\right\rangle_{A}=-i \epsilon_{i j} k^{j} f_{1}^{f}\left(\alpha_{q}, k_{\perp}\right) \\
\frac{1}{8 \pi^{3} s} \int d x_{\bullet} d x_{\perp} e^{-i \alpha_{q} x_{\bullet}+i(k, x)_{\perp}}\left\langle\bar{\psi}^{f}\left(x_{\bullet}, x_{\perp}\right) \breve{A}_{i}(0) \not p_{2} \gamma_{5} \psi^{f}(0)\right\rangle_{A}=i \epsilon_{i j} k^{j} f_{1}^{f}\left(\alpha_{q}, k_{\perp}\right)
\end{gathered}
$$

Finally, we need

$$
\begin{aligned}
& \frac{1}{8 \pi^{3} s} \int d x_{\bullet} d x_{\perp} e^{-i \alpha_{q} x_{\bullet}+i(k, x) \perp}\left\langle\hat{\psi}\left(x_{\bullet}, x_{\perp}\right) \not A\left(x_{\bullet}, x_{\perp}\right) \not p_{2} \not A(0) \hat{\psi}(0)\right\rangle_{A} \\
& =\frac{1}{8 \pi^{3} s} \int d x_{\bullet} d x_{\perp} e^{-i \alpha_{q} x_{\bullet}+i(k, x)_{\perp}}\left\langle\hat{\psi}\left(x_{\bullet}, x_{\perp}\right)\left(\not k_{\perp}+i \overleftarrow{D D}\right) \not p_{2}\left(\not k_{\perp}-i \not D\right) \hat{\psi}(0)\right\rangle_{A} \\
& =\frac{k_{\perp}^{2}}{16 \pi^{3}} f_{1}\left(\alpha_{q}, k_{\perp}\right)+O\left(\alpha_{q}, \beta_{q}\right)
\end{aligned}
$$


and similarly

$$
\begin{aligned}
& \frac{1}{8 \pi^{3} s} \int d x_{\bullet} d x_{\perp} e^{-i \alpha_{q} x_{\bullet}+i(k, x)_{\perp}}\left\langle\hat{\psi}\left(x_{\bullet}, x_{\perp}\right) \mathcal{A}\left(x_{\bullet}, x_{\perp}\right) \sigma_{\star i} \mathcal{A}(0) \hat{\psi}(0)\right\rangle_{A} \\
& =\frac{1}{8 \pi^{3} s} \int d x_{\bullet} d x_{\perp} e^{-i \alpha_{q} x_{\bullet}+i(k, x)_{\perp}}\left\langle\hat{\psi}\left(x_{\bullet}, x_{\perp}\right)\left(k_{\perp}+i \overleftarrow{D D}\right) \sigma_{\star i}\left(\not k_{\perp}-i \not D\right) \hat{\psi}(0)\right\rangle_{A} \\
& =\frac{1}{16 \pi^{3}} \frac{k_{i} k_{\perp}^{2}}{m} h_{1}^{\perp}\left(\alpha_{q}, k_{\perp}\right)+O\left(\alpha_{q}, \beta_{q}\right)
\end{aligned}
$$

For corresponding antiquark distributions we get

$$
\begin{aligned}
& \frac{g}{8 \pi^{3} s} \int d x_{\bullet} d x_{\perp} e^{-i \alpha_{q} x_{\bullet}+i(k, x)_{\perp}}\left\langle\hat{\psi}(0) \not A(0) \not p_{2} A\left(x_{\bullet}, x_{\perp}\right) \hat{\psi}\left(x_{\bullet}, x_{\perp}\right)\right\rangle_{A} \\
& =-\frac{k_{\perp}^{2}}{16 \pi^{3}} \bar{f}_{1}\left(\alpha_{q}, k_{\perp}\right)+O\left(\alpha_{q}, \beta_{q}\right) \\
& \frac{g}{8 \pi^{3} s} \int d x_{\bullet} d x_{\perp} e^{-i \alpha_{q} x_{\bullet}+i(k, x)_{\perp}}\left\langle\hat{\psi}\left(x_{\bullet}, x_{\perp}\right) \not A\left(x_{\bullet}, x_{\perp}\right) \sigma_{\star i} \mathcal{A}(0) \hat{\psi}(0)\right\rangle_{A} \\
& =-\frac{1}{16 \pi^{3}} \frac{k_{i} k_{\perp}^{2}}{m} h_{1}^{\perp}\left(\alpha_{q}, k_{\perp}\right)+O\left(\alpha_{q}, \beta_{q}\right)
\end{aligned}
$$

Also, as we saw in section 4.3.2, at the leading order in $N_{c}$ there is one quark-antiquarkgluon operator that does not reduce to twist-2 distributions. It can be parametrized as follows

$$
\begin{aligned}
& \frac{1}{16 \pi^{3}} \frac{2}{s} \int d x_{\bullet} d^{2} x_{\perp} e^{-i \alpha x_{\bullet}+i(k, x)_{\perp}}\left\langle\hat{\bar{\psi}}_{f}\left(x_{\bullet}, x_{\perp}\right)\left[A_{i}(x) \sigma_{\star j}-\frac{1}{2} g_{i j} A^{k} \sigma_{\star k}(x)\right] \hat{\psi}_{f}(0)\right\rangle_{A} \\
& =-\left(k_{i} k_{j}+\frac{1}{2} g_{i j} k_{\perp}^{2}\right) \frac{1}{m} h_{A}^{f}\left(\alpha, k_{\perp}\right) \\
& \frac{1}{16 \pi^{3}} \frac{2}{s} \int d x_{\bullet} d^{2} x_{\perp} e^{-i \alpha x_{\bullet}+i(k, x)_{\perp}}\left\langle\hat{\bar{\psi}}_{f}(0)\left[A_{i}(0) \sigma_{\bullet j}-\frac{1}{2} g_{i j} A^{k} \sigma_{\bullet k}(0)\right] \hat{\psi}_{f}\left(x_{\bullet}, x_{\perp}\right)\right\rangle_{A} \\
& =-\left(k_{i} k_{j}+\frac{1}{2} g_{i j} k_{\perp}^{2}\right) \frac{1}{m} \bar{h}_{A}^{f}\left(\alpha, k_{\perp}\right)
\end{aligned}
$$

and similarly for the target matrix element.

\section{A.4 Matrix elements for exchange-type power corrections}

We parametrize "exchange" TMDs as follows

$$
\begin{aligned}
& \frac{1}{4 \pi^{3} s^{2}} \int d^{2} x_{\perp} d x_{\bullet} e^{-i \alpha x_{\bullet}+i(k, x)_{\perp}} \int_{-\infty}^{x_{\bullet}} d x_{\bullet}^{\prime}\left\langle\bar{\psi}\left(x_{\bullet}, x_{\perp}\right) \breve{F}_{\star i}(0) \not p_{2} \psi\left(x_{\bullet}^{\prime}, x_{\perp}\right)\right\rangle_{A}=k_{i} j_{1}\left(\alpha, k_{\perp}\right), \\
& \frac{1}{4 \pi^{3} s^{2}} \int d^{2} x_{\perp} d x_{\bullet} e^{-i \alpha x_{\bullet}+i(k, x)_{\perp}} \int_{-\infty}^{x_{\bullet}} d x_{\bullet}^{\prime}\left\langle\bar{\psi}\left(x_{\bullet}, x_{\perp}\right) p_{2} \breve{F}_{\star i}(0) \psi\left(x_{\bullet}^{\prime}, x_{\perp}\right)\right\rangle_{A}=k_{i} j_{2}\left(\alpha, k_{\perp}\right), \\
& \frac{1}{4 \pi^{3} s^{2}} \int d^{2} x_{\perp} d x_{\bullet} e^{-i \alpha x_{\bullet}+i(k, x)_{\perp}} \int_{-\infty}^{x_{\bullet}} d x_{\bullet}^{\prime}\left\langle\bar{\psi}\left(x_{\bullet}^{\prime}, x_{\perp}\right) \not p_{2} \breve{F}_{\star i}(0) \psi\left(x_{\bullet}, x_{\perp}\right)\right\rangle_{A}=k_{i} \bar{j}_{1}\left(\alpha, k_{\perp}\right), \\
& \frac{1}{4 \pi^{3} s^{2}} \int d^{2} x_{\perp} d x_{\bullet} e^{-i \alpha x_{\bullet}+i(k, x)_{\perp}} \int_{-\infty}^{x_{\bullet}} d x_{\bullet}^{\prime}\left\langle\bar{\psi}\left(x_{\bullet}^{\prime}, x_{\perp}\right) \breve{F}_{\star i}(0) \not p_{2} \psi\left(x_{\bullet}, x_{\perp}\right)\right\rangle_{A}=k_{i} \bar{j}_{2}\left(\alpha, k_{\perp}\right)
\end{aligned}
$$


where $\breve{F}_{\mu \nu} \equiv F_{\mu \nu}-i \gamma_{5} \tilde{F}_{\mu \nu}$. By complex conjugation we get

$$
\begin{aligned}
& \frac{1}{4 \pi^{3} s^{2}} \int d^{2} x_{\perp} d x_{\bullet} e^{-i \alpha x_{\bullet}+i(k, x)_{\perp}} \int_{-\infty}^{0} d x_{\bullet}^{\prime}\left\langle\bar{\psi}\left(x_{\bullet}^{\prime}, 0_{\perp}\right) \not p_{2} \breve{F}_{\star i}\left(x_{\bullet}, x_{\perp}\right) \psi(0)\right\rangle_{A}=k_{i} j_{1}^{*}\left(\alpha, k_{\perp}\right), \\
& \frac{1}{4 \pi^{3} s^{2}} \int d^{2} x_{\perp} d x_{\bullet} e^{-i \alpha x_{\bullet}+i(k, x)_{\perp}} \int_{-\infty}^{0} d x_{\bullet}^{\prime}\left\langle\bar{\psi}\left(x_{\bullet}^{\prime}, 0_{\perp}\right) \breve{F}_{\star i}\left(x_{\bullet}, x_{\perp}\right) \not p_{2} \psi(0)\right\rangle_{A}=k_{i} j_{2}^{*}\left(\alpha, k_{\perp}\right), \\
& \frac{1}{4 \pi^{3} s^{2}} \int d^{2} x_{\perp} d x \bullet e^{-i \alpha x_{\bullet}+i(k, x)_{\perp}} \int_{-\infty}^{0} d x_{\bullet}^{\prime}\left\langle\bar{\psi}(0) \breve{F}_{\star i}\left(x_{\bullet}, x_{\perp}\right) \not p_{2} \psi\left(x_{\bullet}^{\prime}, 0_{\perp}\right)\right\rangle_{A}=k_{i} \bar{j}_{1}^{*}\left(\alpha, k_{\perp}\right), \\
& \left.\frac{1}{4 \pi^{3} s^{2}} \int d^{2} x_{\perp} d x_{\bullet} e^{-i \alpha x_{\bullet}+i(k, x)_{\perp}} \int_{-\infty}^{0} d x_{\bullet}^{\prime}\left\langle\bar{\psi}(0) \not p_{2} \breve{F}_{\star i}\left(x_{\bullet}, x_{\perp}\right)\right) \psi\left(x_{\bullet}^{\prime}, 0_{\perp}\right)\right\rangle_{A}=k_{i} \bar{j}_{2}^{*}\left(\alpha, k_{\perp}\right)
\end{aligned}
$$

Note that unlike two-quark matrix elements, quark-quark-gluon ones may have imaginary parts. Target matrix elements are obtained by usual substitutions $\alpha \leftrightarrow \beta, \not p_{2} \leftrightarrow \not p_{1}$, $x_{\bullet} \leftrightarrow x_{\star}$, and $\breve{F}_{\star i} \leftrightarrow \breve{F}_{\bullet i .}{ }^{13}$

Let us now consider the corresponding matrix elements from section 4.4. As shown in ref. [16], eqs. (A.64) and (A.65) lead to (here $\left.x=\left(x_{\bullet}, x_{\perp}\right)\right)$

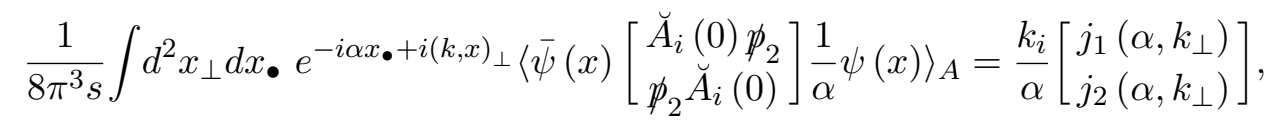

$$
\begin{aligned}
& \frac{1}{8 \pi^{3} s} \int d^{2} x_{\perp} d x_{\bullet} e^{-i \alpha x_{\bullet}+i(k, x)} \perp\left\langle\left(\bar{\psi} \frac{1}{\alpha}\right)\left(x_{\bullet}, x_{\perp}\right)\left[\begin{array}{c}
\not p_{2} \breve{A}_{i}(0) \\
A_{i}(0) \not p_{2}
\end{array}\right] \psi\left(x_{\bullet}, x_{\perp}\right)\right\rangle_{A}=-\frac{k_{i}}{\alpha}\left[\begin{array}{c}
\bar{j}_{1}\left(\alpha, k_{\perp}\right) \\
\bar{j}_{2}\left(\alpha, k_{\perp}\right)
\end{array}\right] \\
& \frac{1}{8 \pi^{3} s} \int d x_{\bullet} e^{-i \alpha x_{\bullet}+i(k, x)_{\perp}}\left\langle\bar{\psi}(0)\left[\begin{array}{l}
\breve{A}_{i}(x) \not p_{2} \\
\not p_{2} \breve{A}_{i}(x)
\end{array}\right] \frac{1}{\alpha} \psi(0)\right\rangle_{A}=-\frac{k_{i}}{\alpha}\left[\begin{array}{l}
\bar{j}_{1}^{*}\left(\alpha, k_{\perp}\right) \\
\bar{j}_{2}^{*}\left(\alpha, k_{\perp}\right)
\end{array}\right], \\
& \frac{1}{8 \pi^{3} s} \int d^{2} x_{\perp} d x_{\bullet} e^{-i \alpha x_{\bullet}+i(k, x) \perp}\left\langle\left(\bar{\psi} \frac{1}{\alpha}\right)(0)\left[\begin{array}{c}
\not p_{2} \breve{A}_{i}(x) \\
A_{i}(x) \not p_{2}
\end{array}\right] \psi(0)\right\rangle_{A}=\frac{k_{i}}{\alpha}\left[\begin{array}{c}
j_{1}^{*}\left(\alpha, k_{\perp}\right) \\
j_{2}^{*}\left(\alpha, k_{\perp}\right)
\end{array}\right]
\end{aligned}
$$

For the target matrix elements, we obtain $\left(x \equiv x_{\star}, x_{\perp}\right)$

$$
\begin{aligned}
& \frac{1}{8 \pi^{3} s} \int d^{2} x_{\perp} d x_{\star} e^{-i \beta x_{\star}+i(k, x) \perp}\left\langle\bar{\psi}(x)\left[\begin{array}{l}
\breve{B}_{i}(0) \not p_{1} \\
\not \not_{1} \breve{B}_{i}(0)
\end{array}\right] \frac{1}{\beta} \psi(x\rangle_{B}=\frac{k_{i}}{\beta}\left[\begin{array}{l}
j_{1}\left(\beta, k_{\perp}\right) \\
j_{2}\left(\beta, k_{\perp}\right)
\end{array}\right],\right. \\
& \frac{1}{8 \pi^{3} s} \int d^{2} x_{\perp} d x_{\star} e^{-i \alpha x_{\star}+i(k, x) \perp}\left\langle\left(\bar{\psi} \frac{1}{\beta}\right)(x)\left[\begin{array}{c}
\not p_{1} \breve{B}_{i}(0) \\
\breve{B}_{i}(0) \not p_{1}
\end{array}\right] \psi(x)\right\rangle_{B}=-\frac{k_{i}}{\beta}\left[\begin{array}{l}
\bar{j}_{1}\left(\beta, k_{\perp}\right) \\
\bar{j}_{2}\left(\beta, k_{\perp}\right)
\end{array}\right], \\
& \frac{1}{8 \pi^{3} s} \int d^{2} x_{\perp} d x_{\star} e^{-i \beta x_{\star}+i(k, x)_{\perp}}\left\langle\bar{\psi}(0)\left[\begin{array}{l}
\breve{B}_{i}(x) \not p_{1} \\
\not p_{1} \breve{B}_{i}(x)
\end{array}\right] \frac{1}{\beta} \psi(0)\right\rangle_{B}=-\frac{k_{i}}{\beta}\left[\begin{array}{l}
\bar{j}_{1}^{*}\left(\beta, k_{\perp}\right) \\
\bar{j}_{2}^{*}\left(\beta, k_{\perp}\right)
\end{array}\right], \\
& \frac{1}{8 \pi^{3} s} \int d^{2} x_{\perp} d x_{\star} e^{-i \beta x_{\star}+i(k, x)_{\perp}}\left\langle\left(\bar{\psi} \frac{1}{\beta}\right)(0)\left[\begin{array}{c}
\not p_{1} \breve{B}_{i}(x) \\
\breve{B}_{i}(x) \not p_{1}
\end{array}\right] \psi(0)\right\rangle_{B}=\frac{k_{i}}{\beta}\left[\begin{array}{c}
j_{1}^{*}\left(\beta, k_{\perp}\right) \\
j_{2}^{*}\left(\beta, k_{\perp}\right)
\end{array}\right]
\end{aligned}
$$

\footnotetext{
${ }^{13}$ For completeness let us present the explicit form of the gauge links in an arbitrary gauge:

$$
\begin{aligned}
& \bar{\psi}\left(x_{\bullet}^{\prime}, x_{\perp}\right) F_{\star i}(0) \psi\left(x_{\bullet}, x_{\perp}\right) \rightarrow \bar{\psi}\left(x_{\bullet}^{\prime}, x_{\perp}\right)\left[x_{\bullet}^{\prime},-\infty\right]_{x}\left[x_{\perp}, 0_{\perp}\right]_{-\infty} . \\
& \times\left[-\infty_{\bullet}, 0\right]_{\perp} F_{\star i}(0)\left[0,-\infty_{\bullet}\right]_{\perp}\left[0_{\perp}, x_{\perp}\right]_{-\infty}\left[-\infty_{\bullet}, x_{\bullet}\right]_{x} \psi\left(x_{\bullet}, x_{\perp}\right) .
\end{aligned}
$$
}


We need also parametrization of matrix elements with extra $\gamma_{5}$. Since $\breve{A}_{i} \gamma_{5}=i \epsilon_{i j} \breve{A}^{j}$ and $\breve{B}_{i} \gamma_{5}=i \epsilon_{i j} \breve{B}^{j}$ we get from eqs. (A.66), (A.67)

$$
\begin{aligned}
& \frac{1}{8 \pi^{3} s} \int d^{2} x_{\perp} d x_{\bullet} e^{-i \alpha x_{\bullet}+i(k, x) \perp}\left\langle\bar{\psi}(x)\left[\begin{array}{l}
\breve{A}_{i}(0) \not p_{2} \\
\not p_{2} \breve{A}_{i}(0)
\end{array}\right] \frac{\gamma_{5}}{\alpha} \psi\left(x_{\bullet}, x_{\perp}\right)\right\rangle_{A}=i \epsilon_{i j} \frac{k^{j}}{\alpha}\left[\begin{array}{c}
-j_{1}\left(\alpha, k_{\perp}\right) \\
j_{2}\left(\alpha, k_{\perp}\right)
\end{array}\right], \\
& \frac{1}{8 \pi^{3} s} \int d^{2} x_{\perp} d x_{\bullet} e^{-i \alpha x_{\bullet}+i(k, x)_{\perp}}\left\langle\left(\bar{\psi} \frac{1}{\alpha}\right)(x)\left[\begin{array}{c}
\not p_{2} \breve{A}_{i}(0) \\
\vec{A}_{i}(0) \not p_{2}
\end{array}\right] \gamma_{5} \psi(x)\right\rangle_{A}=i \epsilon_{i j} \frac{k^{j}}{\alpha}\left[\begin{array}{c}
-\bar{j}_{1}\left(\alpha, k_{\perp}\right) \\
\bar{j}_{2}\left(\alpha, k_{\perp}\right)
\end{array}\right] \\
& \frac{1}{8 \pi^{3} s} \int d x_{\bullet} e^{-i \alpha x_{\bullet}+i(k, x)_{\perp}}\left\langle\bar{\psi}(0)\left[\begin{array}{c}
\breve{A}_{i}(x) \not p_{2} \\
\not p_{2} \breve{A}_{i}(x)
\end{array}\right] \frac{\gamma_{5}}{\alpha} \psi(0)\right\rangle_{A}=i \epsilon_{i j} \frac{k^{j}}{\alpha}\left[\begin{array}{c}
\bar{j}_{1}^{*}\left(\alpha, k_{\perp}\right) \\
-\bar{j}_{2}^{*}\left(\alpha, k_{\perp}\right)
\end{array}\right], \\
& \frac{1}{8 \pi^{3} s} \int d^{2} x_{\perp} d x_{\bullet} e^{-i \alpha x_{\bullet}+i(k, x)_{\perp}}\left\langle\left(\bar{\psi} \frac{1}{\alpha}\right)(0)\left[\begin{array}{c}
\not \not_{2} \breve{A}_{i}(x) \\
A_{i}(x) \not p_{2}
\end{array}\right] \gamma_{5} \psi(0)\right\rangle_{A}=i \epsilon_{i j} \frac{k^{j}}{\alpha}\left[\begin{array}{c}
j_{1}^{*}\left(\alpha, k_{\perp}\right) \\
-j_{2}^{*}\left(\alpha, k_{\perp}\right)
\end{array}\right] \text {, }
\end{aligned}
$$

$$
\begin{aligned}
& \frac{1}{8 \pi^{3} s} \int d^{2} x_{\perp} d x_{\bullet} e^{-i \alpha x \cdot+i(k, x)_{\perp}}\left\langle\bar{\psi}(x)\left[\begin{array}{l}
\breve{A}_{i}(0) \not p_{2} \\
\not \not_{2} \breve{A}_{i}(0)
\end{array}\right] \frac{\gamma_{5}}{\alpha} \psi(x)\right\rangle_{A}=i \epsilon_{i j} \frac{k^{j}}{\alpha}\left[\begin{array}{c}
-j_{1}\left(\alpha, k_{\perp}\right) \\
j_{2}\left(\alpha, k_{\perp}\right)
\end{array}\right], \\
& \frac{1}{8 \pi^{3} s} \int d^{2} x_{\perp} d x_{\bullet} e^{-i \alpha x_{\bullet}+i(k, x)_{\perp}}\left\langle\left(\bar{\psi} \frac{1}{\alpha}\right)(x)\left[\begin{array}{c}
\not p_{2} \breve{A}_{i}(0) \\
\vec{A}_{i}(0) \not p_{2}
\end{array}\right] \gamma_{5} \psi(x)\right\rangle_{A}=i \epsilon_{i j} \frac{k^{j}}{\alpha}\left[\begin{array}{c}
-\bar{j}_{1}\left(\alpha, k_{\perp}\right) \\
\bar{j}_{2}\left(\alpha, k_{\perp}\right)
\end{array}\right] \\
& \frac{1}{8 \pi^{3} s} \int d x_{\bullet} e^{-i \alpha x \bullet+i(k, x)_{\perp}}\left\langle\bar{\psi}(0)\left[\begin{array}{c}
\breve{A}_{i}(x) \not p_{2} \\
\not p_{2} \breve{A}_{i}(x)
\end{array}\right] \frac{\gamma_{5}}{\alpha} \psi(0)\right\rangle_{A}=i \epsilon_{i j} \frac{k^{j}}{\alpha}\left[\begin{array}{c}
\bar{j}_{1}^{*}\left(\alpha, k_{\perp}\right) \\
-\bar{j}_{2}^{*}\left(\alpha, k_{\perp}\right)
\end{array}\right], \\
& \frac{1}{8 \pi^{3} s} \int d^{2} x_{\perp} d x_{\bullet} e^{-i \alpha x_{\bullet}+i(k, x)_{\perp}}\left\langle\left(\bar{\psi} \frac{1}{\alpha}\right)(0)\left[\begin{array}{c}
\not p_{2} \breve{A}_{i}(x) \\
A_{i}(x) \not p_{2}
\end{array}\right] \gamma_{5} \psi(0)\right\rangle_{A}=i \epsilon_{i j} \frac{k^{j}}{\alpha}\left[\begin{array}{c}
j_{1}^{*}\left(\alpha, k_{\perp}\right) \\
-j_{2}^{*}\left(\alpha, k_{\perp}\right)
\end{array}\right]
\end{aligned}
$$

where $x \equiv x_{\bullet}, x_{*}$, and

$$
\begin{aligned}
& \frac{1}{8 \pi^{3} s} \int d^{2} x_{\perp} d x_{\star} e^{-i \beta x_{\star}+i(k, x)_{\perp}}\left\langle\bar{\psi}(x)\left[\begin{array}{c}
\breve{B}_{i}(0) \not p_{1} \\
\not \not_{1} \breve{B}_{i}(0)
\end{array}\right] \frac{\gamma_{5}}{\beta} \psi(x)\right\rangle_{B}=i \epsilon_{i j} \frac{k^{j}}{\beta}\left[\begin{array}{c}
-j_{1}\left(\beta, k_{\perp}\right) \\
j_{2}\left(\beta, k_{\perp}\right)
\end{array}\right], \\
& \frac{1}{8 \pi^{3} s} \int d^{2} x_{\perp} d x_{\star} e^{-i \alpha x_{\star}+i(k, x)_{\perp}}\left\langle\left(\bar{\psi} \frac{1}{\beta}\right)(x)\left[\begin{array}{c}
\not p_{1} \breve{B}_{i}(0) \\
\breve{B}_{i}(0) \not p_{1}
\end{array}\right] \gamma_{5} \psi(x)\right\rangle_{B}=i \epsilon_{i j} \frac{k^{j}}{\beta}\left[\begin{array}{c}
-\bar{j}_{1}\left(\beta, k_{\perp}\right) \\
\bar{j}_{2}\left(\beta, k_{\perp}\right)
\end{array}\right] \text {, } \\
& \frac{1}{8 \pi^{3} s} \int d^{2} x_{\perp} d x_{\star} e^{-i \beta x_{\star}+i(k, x)_{\perp}}\left\langle\bar{\psi}(0)\left[\begin{array}{c}
\breve{B}_{i}(x) \not p_{1} \\
\not p_{1} \breve{B}_{i}(x)
\end{array}\right] \frac{\gamma_{5}}{\beta} \psi(0)\right\rangle_{B}=i \epsilon_{i j} \frac{k^{j}}{\beta}\left[\begin{array}{c}
\bar{j}_{1}^{*}\left(\beta, k_{\perp}\right) \\
-\bar{j}_{2}^{*}\left(\beta, k_{\perp}\right)
\end{array}\right], \\
& \frac{1}{8 \pi^{3} s} \int d^{2} x_{\perp} d x_{\star} e^{-i \beta x_{\star}+i(k, x)_{\perp}}\left\langle\left(\bar{\psi} \frac{1}{\beta}\right)(0)\left[\begin{array}{c}
\not p_{1} \breve{B}_{i}(x) \\
\breve{B}_{i}(x) \not p_{1}
\end{array}\right] \gamma_{5} \psi(0)\right\rangle_{B}=i \epsilon_{i j} \frac{k^{j}}{\beta}\left[\begin{array}{c}
j_{1}^{*}\left(\beta, k_{\perp}\right) \\
-j_{2}^{*}\left(\beta, k_{\perp}\right)
\end{array}\right]
\end{aligned}
$$

where $x \equiv x_{\star}, x_{*}$ 
It is convenient to introduce the following combinations for the parametrization of the exchange-type power corrections

$$
\begin{aligned}
& J_{++}^{1 f f^{\prime}}\left(q, k_{\perp}\right)=\left(j_{1}+\bar{j}_{1}\right)^{f}\left(\alpha_{q}, k_{\perp}\right)\left(j_{1}^{*}+\bar{j}_{1}^{*}\right)^{f^{\prime}}\left(\beta_{q},(q-k)_{\perp}\right)+\text { c.c. }, \\
& J_{++}^{2 f f^{\prime}}\left(q, k_{\perp}\right)=\left(j_{2}+\bar{j}_{2}\right)^{f}\left(\alpha_{q}, k_{\perp}\right)\left(j_{2}^{*}+\bar{j}_{2}^{*}\right)^{f^{\prime}}\left(\beta_{q},(q-k)_{\perp}\right)+c . c ., \\
& J_{--}^{1 f f^{\prime}}\left(q, k_{\perp}\right)=\left(j_{1}-\bar{j}_{1}\right)^{f}\left(\alpha_{q}, k_{\perp}\right)\left(j_{1}^{*}-\bar{j}_{1}^{*}\right)^{f^{\prime}}\left(\beta_{q},(q-k)_{\perp}\right)+c . c ., \\
& J_{--}^{2 f f^{\prime}}\left(q, k_{\perp}\right)=\left(j_{2}-\bar{j}_{2}\right)^{f}\left(\alpha_{q}, k_{\perp}\right)\left(j_{2}^{*}-\bar{j}_{2}^{*}\right)^{f^{\prime}}\left(\beta_{q},(q-k)_{\perp}\right)+c . c ., \\
& J_{+-}^{1 f f^{\prime}}\left(q, k_{\perp}\right)=\left(j_{1}+\bar{j}_{1}\right)^{f}\left(\alpha_{q}, k_{\perp}\right)\left(j_{1}^{*}-\bar{j}_{1}^{*}\right)^{f^{\prime}}\left(\beta_{q},(q-k)_{\perp}\right)+c . c ., \\
& J_{-+}^{1 f f^{\prime}}\left(q, k_{\perp}\right)=\left(j_{1}-\bar{j}_{1}\right)^{f}\left(\alpha_{q}, k_{\perp}\right)\left(j_{1}^{*}+\bar{j}_{1}^{*}\right)^{f^{\prime}}\left(\beta_{q},(q-k)_{\perp}\right)+c . c ., \\
& J_{+-}^{2 f f^{\prime}}\left(q, k_{\perp}\right)=\left(j_{2}+\bar{j}_{2}\right)^{f}\left(\alpha_{q}, k_{\perp}\right)\left(j_{2}^{*}-\bar{j}_{2}^{*}\right)^{f^{\prime}}\left(\beta_{q},(q-k)_{\perp}\right)+c . c ., \\
& J_{-+}^{2 f f^{\prime}}\left(q, k_{\perp}\right)=\left(j_{2}-\bar{j}_{2}\right)^{f}\left(\alpha_{q}, k_{\perp}\right)\left(j_{2}^{*}+\bar{j}_{2}^{*}\right)^{f^{\prime}}\left(\beta_{q},(q-k)_{\perp}\right)+\text { c.c. },
\end{aligned}
$$

and

$$
\begin{aligned}
& I_{--}^{1 f f^{\prime}}\left(q, k_{\perp}\right)=i\left(j_{1}-\bar{j}_{1}\right)^{f}\left(\alpha_{q}, k_{\perp}\right)\left(j_{1}^{*}-\bar{j}_{1}^{*}\right)^{f^{\prime}}\left(\beta_{q},(q-k)_{\perp}\right)+\text { c.c., } \\
& I_{--}^{2 f f^{\prime}}\left(q, k_{\perp}\right)=i\left(j_{2}-\bar{j}_{2}\right)^{f}\left(\alpha_{q}, k_{\perp}\right)\left(j_{2}^{*}-\bar{j}_{2}^{*}\right)^{f^{\prime}}\left(\beta_{q},(q-k)_{\perp}\right)+\text { c.c., } \\
& I_{+-}^{1 f f^{\prime}}\left(q, k_{\perp}\right)=i\left(j_{1}+\bar{j}_{1}\right)^{f}\left(\alpha_{q}, k_{\perp}\right)\left(j_{1}^{*}-\bar{j}_{1}^{*}\right)^{f^{\prime}}\left(\beta_{q},(q-k)_{\perp}\right)+\text { c.c., } \\
& I_{-+}^{1 f f^{\prime}}\left(q, k_{\perp}\right)=i\left(j_{1}-\bar{j}_{1}\right)^{f}\left(\alpha_{q}, k_{\perp}\right)\left(j_{1}^{*}+\bar{j}_{1}^{*}\right)^{f^{\prime}}\left(\beta_{q},(q-k)_{\perp}\right)+\text { c.c., } \\
& I_{+-}^{2 f f^{\prime}}\left(q, k_{\perp}\right)=i\left(j_{2}+\bar{j}_{2}\right)^{f}\left(\alpha_{q}, k_{\perp}\right)\left(j_{2}^{*}-\bar{j}_{2}^{*}\right)^{f^{\prime}}\left(\beta_{q},(q-k)_{\perp}\right)+\text { c.c. } \\
& I_{-+}^{2 f f^{\prime}}\left(q, k_{\perp}\right)=i\left(j_{2}-\bar{j}_{2}\right)^{f}\left(\alpha_{q}, k_{\perp}\right)\left(j_{2}^{*}+\bar{j}_{2}^{*}\right)^{f^{\prime}}\left(\beta_{q},(q-k)_{\perp}\right)+\text { c.c. }
\end{aligned}
$$

\section{A.5 Products of leptonic tensor and hadronic tensors' structures}

The lepton momenta in the Collins-Soper frame are parametrized as

$l=\frac{Q}{2}(1, \sin \theta \cos \phi, \sin \theta \sin \phi, \cos \theta), \quad l^{\prime}=\frac{Q}{2}(1,-\sin \theta \cos \phi,-\sin \theta \sin \phi,-\cos \theta)$

The products of the symmetric leptonic tensor with unit vectors $X, Y$, and $Z$ are

$$
\begin{aligned}
L^{\mu \nu}\left(\frac{q_{\mu} q_{\nu}}{q^{2}}-g_{\mu \nu}\right) & =2 \frac{(q \cdot l)\left(q \cdot l^{\prime}\right)}{q^{2}}+l \cdot l^{\prime}=Q^{2} \\
L^{\mu \nu} X_{\mu} X_{\nu} & =2(l \cdot X)\left(l^{\prime} \cdot X\right)+l \cdot l^{\prime}=\frac{Q^{2}}{2}\left[-\sin ^{2} \theta \cos ^{2} \phi+1\right] \\
L^{\mu \nu} Z_{\mu} Z_{\nu} & =2(l \cdot Z)\left(l^{\prime} \cdot Z\right)+l \cdot l^{\prime}=\frac{Q^{2}}{2}\left[-\cos ^{2} \theta+1\right]=\frac{Q^{2}}{2} \sin ^{2} \theta \\
L^{\mu \nu} X_{\mu} Z_{\nu} & =(l \cdot X)\left(l^{\prime} \cdot Z\right)+\left(l^{\prime} \cdot X\right)(l \cdot Z)=-\frac{Q^{2}}{4} \sin 2 \theta \cos \phi \\
L^{\mu \nu} Y_{\mu} Z_{\nu} & =(l \cdot Y)\left(l^{\prime} \cdot Z\right)+\left(l^{\prime} \cdot Y\right)(l \cdot Z)=-\frac{Q^{2}}{4} \sin 2 \theta \sin \phi \\
L^{\mu \nu} Y_{\mu} X_{\nu} & =(l \cdot Y)\left(l^{\prime} \cdot X\right)+\left(l^{\prime} \cdot Y\right)(l \cdot X)=-\frac{Q^{2}}{4} \sin ^{2} \theta \sin 2 \phi
\end{aligned}
$$


and therefore

$$
\begin{aligned}
& \frac{1}{Q^{2}} L^{\mu \nu}\left(-\left(g_{\mu \nu}-\frac{q_{\mu} q_{\nu}}{q^{2}}\right)\left(W_{T}+W_{\Delta \Delta}\right)-2 X_{\mu} X_{\nu} W_{\Delta \Delta}\right. \\
& \left.+Z_{\mu} Z_{\nu}\left(W_{L}-W_{T}-W_{\Delta \Delta}\right)-\left(X_{\mu} Z_{\nu}+X_{\nu} Z_{\mu}\right) W_{\Delta}\right) \\
& \quad=\frac{1}{2}\left(W_{T}+W_{L}\right)\left[1+\cos ^{2} \theta \frac{W_{T}-W_{L}}{W_{T}+W_{L}}+\sin 2 \theta \sin \phi \frac{W_{\Delta}}{W_{T}+W_{L}}+\sin ^{2} \theta \cos 2 \phi \frac{W_{\Delta \Delta}}{W_{T}+W_{L}}\right]
\end{aligned}
$$

We also need products of antisymmetric leptonic tensor with hadron sctructures

$$
\begin{aligned}
& \epsilon_{\mu \nu \alpha \beta} l^{\alpha} l^{\prime \beta} \epsilon^{\mu \nu \lambda \rho} q_{\lambda} Z_{\rho} \\
& =-2\left[(l \cdot q)\left(l^{\prime} \cdot Z\right)-\left(l^{\prime} \cdot q\right)(l \cdot Z)\right]=Q\left[(l \cdot Z)-\left(l^{\prime} \cdot Z\right)\right]=-Q^{2} \cos \theta, \\
& \epsilon_{\mu \nu \alpha \beta} l^{\alpha} l^{\prime \beta} \epsilon^{\mu \nu \lambda \rho} q_{\lambda} X_{\rho} \\
& =-2\left[(l \cdot q)\left(l^{\prime} \cdot X\right)-\left(l^{\prime} \cdot q\right)(l \cdot X)\right]=Q\left[(l \cdot X)-\left(l^{\prime} \cdot X\right)\right]=-Q^{2} \sin \theta \cos \phi
\end{aligned}
$$

and therefore $\left(Q_{\|}=\sqrt{Q^{2}+Q_{\perp}^{2}}\right)$

$$
\begin{aligned}
& \epsilon^{\mu \nu \alpha \beta} l_{\alpha} l_{\beta}^{\prime} \epsilon_{\mu \nu \lambda \rho} q^{\lambda}\left[\frac{Z^{\rho}}{Q_{\|}} \mathcal{W}_{1}^{\mathrm{F} f}(q)+X^{\rho} \frac{Q_{\perp}}{Q_{\|} Q} \mathcal{W}_{2}^{\mathrm{F} f}(q)\right] \\
& =-Q^{2}\left[\mathcal{W}_{1}^{\mathrm{F} f}(q) \cos \theta+\frac{Q_{\perp}}{Q} \mathcal{W}_{2}^{\mathrm{F} f}(q) \sin \theta \cos \phi\right]\left[1+O\left(\frac{Q_{\perp}^{2}}{Q^{2}}\right)\right] .
\end{aligned}
$$

Open Access. This article is distributed under the terms of the Creative Commons Attribution License (CC-BY 4.0), which permits any use, distribution and reproduction in any medium, provided the original author(s) and source are credited.

\section{References}

[1] S.D. Drell and T.-M. Yan, Massive Lepton Pair Production in Hadron-Hadron Collisions at High-Energies, Phys. Rev. Lett. 25 (1970) 316 [Erratum ibid. 25 (1970) 902] [INSPIRE].

[2] J.C. Collins and D.E. Soper, Angular Distribution of Dileptons in High-Energy Hadron Collisions, Phys. Rev. D 16 (1977) 2219 [InSPIRE].

[3] J.C. Collins, Simple Prediction of QCD for Angular Distribution of Dileptons in Hadron Collisions, Phys. Rev. Lett. 42 (1979) 291 [inSPIRE].

[4] E. Mirkes and J. Ohnemus, $W$ and $Z$ polarization effects in hadronic collisions, Phys. Rev. $D$ 50 (1994) 5692 [hep-ph/9406381] [INSPIRE].

[5] E. Mirkes and J. Ohnemus, Angular distributions of Drell-Yan lepton pairs at the Tevatron: Order $\alpha_{s}^{2}$ corrections and Monte Carlo studies, Phys. Rev. D 51 (1995) 4891 [hep-ph/9412289] [INSPIRE].

[6] E.L. Berger, J.-W. Qiu and R.A. Rodriguez-Pedraza, Angular distribution of leptons from the decay of massive vector bosons, Phys. Lett. B 656 (2007) 74 [arXiv:0707.3150] [InSPIRE].

[7] E.L. Berger, J.-W. Qiu and R.A. Rodriguez-Pedraza, Transverse momentum dependence of the angular distribution of the Drell-Yan process, Phys. Rev. D 76 (2007) 074006 [arXiv:0708.0578] [INSPIRE]. 
[8] M. Lambertsen and W. Vogelsang, Drell-Yan lepton angular distributions in perturbative QCD, Phys. Rev. D 93 (2016) 114013 [arXiv:1605.02625] [INSPIRE].

[9] J. Collins, Foundations of perturbative QCD. Cambridge University Press (2013) [ISBN: 9781107645257].

[10] J.C. Collins and D.E. Soper, Parton Distribution and Decay Functions, Nucl. Phys. B 194 (1982) 445 [INSPIRE].

[11] J.C. Collins, D.E. Soper and G.F. Sterman, Transverse Momentum Distribution in Drell-Yan Pair and $W$ and $Z$ Boson Production, Nucl. Phys. B 250 (1985) 199 [inSPIRE].

[12] X. Ji, J.-P. Ma and F. Yuan, QCD factorization for semi-inclusive deep-inelastic scattering at low transverse momentum, Phys. Rev. D 71 (2005) 034005 [hep-ph/0404183] [INSPIRE].

[13] M.G. Echevarria, A. Idilbi and I. Scimemi, Factorization Theorem For Drell-Yan At Low $q_{T}$ And Transverse Momentum Distributions On-The-Light-Cone, JHEP 07 (2012) 002 [arXiv:1111.4996] [INSPIRE].

[14] I. Balitsky and A. Tarasov, Power corrections to TMD factorization for Z-boson production, JHEP 05 (2018) 150 [arXiv:1712.09389] [INSPIRE].

[15] I. Balitsky and A. Tarasov, Higher-twist corrections to gluon TMD factorization, JHEP 07 (2017) 095 [arXiv: 1706. 01415] [INSPIRE].

[16] I. Balitsky, Gauge-invariant TMD factorization for Drell-Yan hadronic tensor at small $x$, JHEP 05 (2021) 046 [arXiv: 2012.01588] [INSPIRE].

[17] ATLAS collaboration, Measurement of the angular coefficients in Z-boson events using electron and muon pairs from data taken at $\sqrt{s}=8 \mathrm{TeV}$ with the ATLAS detector, JHEP 08 (2016) 159 [arXiv : 1606.00689] [INSPIRE].

[18] CMS collaboration, Angular coefficients of $Z$ bosons produced in pp collisions at $\sqrt{s}=8 \mathrm{TeV}$ and decaying to $\mu^{+} \mu^{-}$as a function of transverse momentum and rapidity, Phys. Lett. $B$ 750 (2015) 154 [arXiv: 1504.03512] [INSPIRE].

[19] M.A. Ebert, J.K.L. Michel, I.W. Stewart and F.J. Tackmann, Drell-Yan $q_{T}$ resummation of fiducial power corrections at $N^{3} L L$, JHEP 04 (2021) 102 [arXiv: 2006.11382] [INSPIRE].

[20] P.J. Mulders and R.D. Tangerman, The Complete tree level result up to order $1 / Q$ for polarized deep inelastic leptoproduction, Nucl. Phys. B 461 (1996) 197 [Erratum ibid. 484 (1997) 538] [hep-ph/9510301] [INSPIRE].

[21] R.D. Tangerman and P.J. Mulders, Intrinsic transverse momentum and the polarized Drell-Yan process, Phys. Rev. D 51 (1995) 3357 [hep-ph/9403227] [INSPIRE].

[22] M. Nefedov and V. Saleev, Off-shell initial state effects, gauge invariance and angular distributions in the Drell-Yan process, Phys. Lett. B 790 (2019) 551 [arXiv:1810.04061] [INSPIRE].

[23] M.A. Nefedov and V.A. Saleev, High-energy factorization for the drell-yan process in pp and $p \bar{p}$ collisions with new unintegrated pdfs, Phys. Rev. D 102 (2020) 114018.

[24] M.A. Nefedov, N.N. Nikolaev and V.A. Saleev, Drell-Yan lepton pair production at high energies in the Parton Reggeization Approach, Phys. Rev. D 87 (2013) 014022 [arXiv: 1211.5539] [INSPIRE].

[25] V.M. Braun and A.N. Manashov, Operator product expansion in QCD in off-forward kinematics: Separation of kinematic and dynamical contributions, JHEP 01 (2012) 085 [arXiv: 1111.6765] [INSPIRE]. 
[26] V.M. Braun and A.N. Manashov, Kinematic power corrections in off-forward hard reactions, Phys. Rev. Lett. 107 (2011) 202001 [arXiv:1108.2394] [InSPIRE].

[27] V.M. Braun, Y. Ji and A.N. Manashov. Two-photon processes in conformal QCD: Resummation of the descendants of leading-twist operators, JHEP 03 (2021) 051.

[28] I. Scimemi and A. Vladimirov, Matching of transverse momentum dependent distributions at twist-3, Eur. Phys. J. C $\mathbf{7 8}$ (2018) 802.

[29] V. Barone, S. Melis and A. Prokudin, The Boer-Mulders effect in unpolarized SIDIS: An Analysis of the COMPASS and HERMES data on the cos 2 phi asymmetry, Phys. Rev. D 81 (2010) 114026 [arXiv:0912.5194] [INSPIRE].

[30] V. Barone, S. Melis and A. Prokudin, Azimuthal asymmetries in unpolarized Drell-Yan processes and the Boer-Mulders distributions of antiquarks, Phys. Rev. D 82 (2010) 114025 [arXiv: 1009.3423] [INSPIRE].

[31] M.G. Echevarria, I. Scimemi and A. Vladimirov, Universal transverse momentum dependent soft function at NNLO, Phys. Rev. D 93 (2016) 054004 [arXiv: 1511.05590] [INSPIRE].

[32] Y. Li, D. Neill and H. X. Zhu, An exponential regulator for rapidity divergences, Nucl. Phys. $B 960$ (2020) 115193.

[33] T. Lübbert, J. Oredsson and M. Stahlhofen, Rapidity renormalized TMD soft and beam functions at two loops, JHEP 03 (2016) 168 [arXiv:1602.01829] [INSPIRE].

[34] M.G. Echevarria, I. Scimemi and A. Vladimirov, Unpolarized Transverse Momentum Dependent Parton Distribution and Fragmentation Functions at next-to-next-to-leading order, JHEP 09 (2016) 004 [arXiv: 1604.07869] [InSPIRE].

[35] Y.V. Kovchegov and M.D. Sievert, Calculating TMDs of a Large Nucleus: Quasi-Classical Approximation and Quantum Evolution, Nucl. Phys. B 903 (2016) 164 [arXiv:1505.01176] [INSPIRE]. 